UNIVERSITY OF ROCHESTER

LABORATORY FOR LASER ENERGETICS
Volume 98

January-March 2004

DOE/SF/19460-527

\title{
LLE Review
}

\section{Quarterly Report}

UR

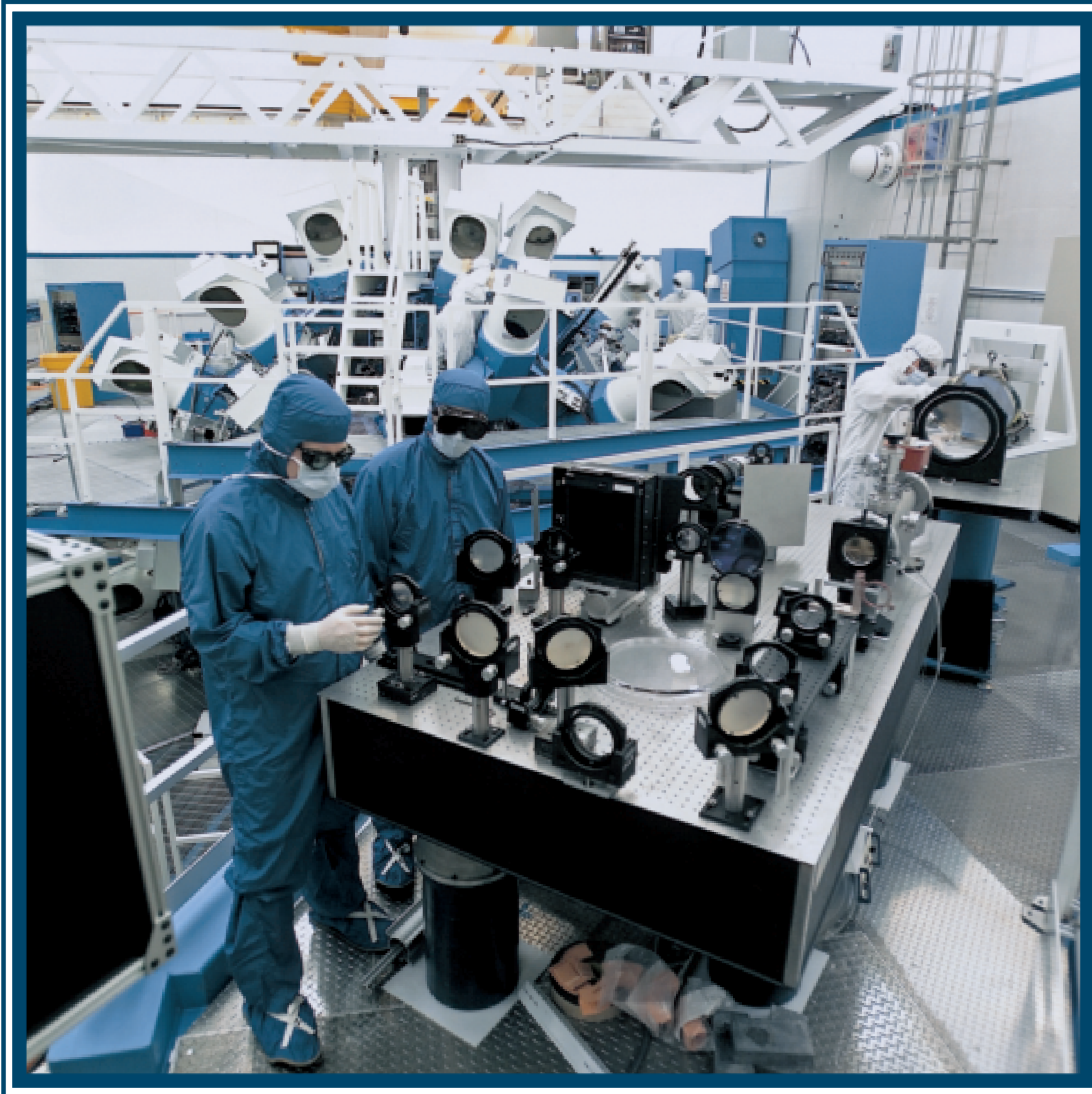




\section{About the Cover:}

The ultraviolet diagnostic table (UVDT) situated on top of the south end-mirror structure in the OMEGA Target Bay is shown in the foreground of the photograph. A full-aperture optical wedge in one of the 60 beams directs $4 \%$ of the laser light to the UVDT. Scientists Sean Regan (left foreground) and John Marozas (right foreground) use the ultraviolet equivalent-target-plane (UVETP) diagnostic stationed on the UVDT to investigate the performance of laser-beam smoothing on OMEGA with 1-THz-bandwidth, 2-D smoothing by spectral dispersion (2-D SSD) and polarization smoothing (PS) (see article featured on p. 49). Optomechanical technician Rich Dean (far right) is seen positioning a distributed phase plate (DPP) in front of an OMEGA lens on the UVDT. The UVETP diagnostic captures a magnified image of the OMEGA far field on a CCD camera, which is located inside the black enclosure on the left side of the photograph. The Optical Manufacturing (OMAN) Group can be seen in the background installing phase plates on the OMEGA target chamber.

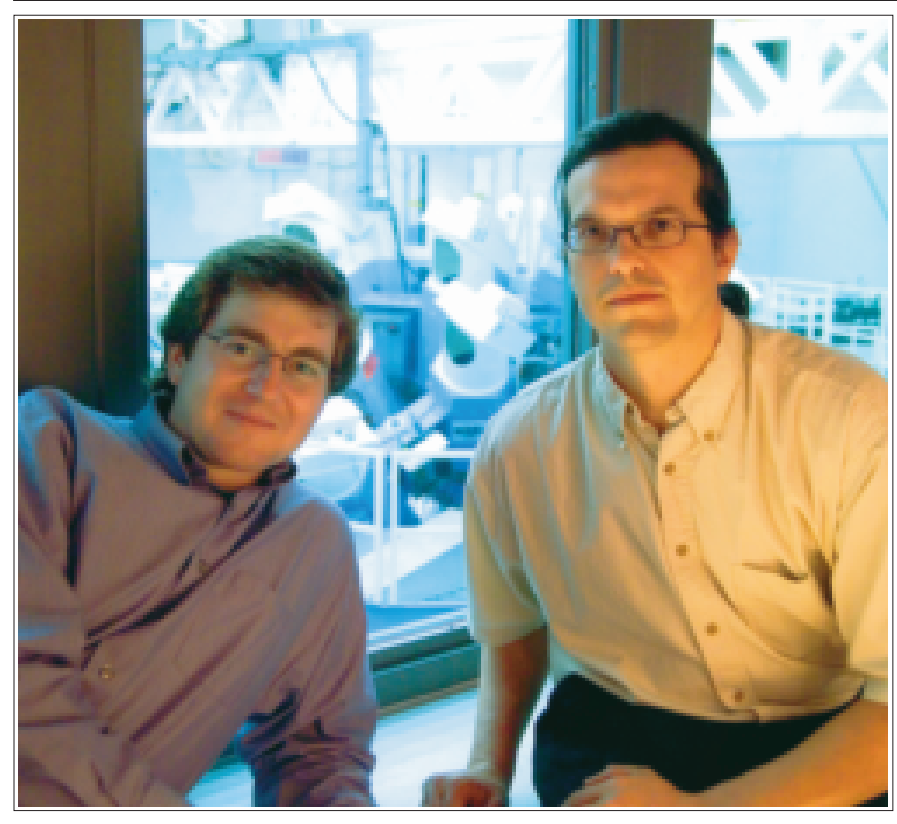

This report was prepared as an account of work conducted by the Laboratory for Laser Energetics and sponsored by New York State Energy Research and Development Authority, the University of Rochester, the U.S. Department of Energy, and other agencies. Neither the above named sponsors, nor any of their employees, makes any warranty, expressed or implied, or assumes any legal liability or responsibility for the accuracy, completeness, or usefulness of any information, apparatus, product, or process disclosed, or represents that its use would not infringe privately owned rights. Reference herein to any specific commercial product, process, or service by trade name, mark, manufacturer, or otherwise, does not necessarily constitute or imply its endorsement, recommendation, or favoring by

\footnotetext{
Printed in the United States of America Available from

National Technical Information Services

U.S. Department of Commerce

5285 Port Royal Road

Springfield, VA 22161
}

In the OMEGA viewing gallery, scientists Sean Regan (left) and John Marozas (right) discuss the on-target laser irradiation nonuniformity levels. Direct-drive inertial confinement fusion strives to achieve uniform target irradiation using two-dimensional smoothing by spectral dispersion (2-D SSD), distributed phase plates (DPP's), polarization smoothing (PS) utilizing birefringent wedges, and multiple-beam overlap. The article featured on p. 49 shows that the theoretical predictions of laser beam smoothing with 1-THz-bandwidth, 2-D SSD and PS are in excellent agreement with the measured performance. the United States Government or any agency thereof or any other sponsor. Results reported in the LLE Review should not be taken as necessarily final results as they represent active research. The views and opinions of authors expressed herein do not necessarily state or reflect those of any of the above sponsoring entities.

The work described in this volume includes current research at the Laboratory for Laser Energetics, which is supported by New York State Energy Research and Development Authority, the University of Rochester, the U.S. Department of Energy Office of Inertial Confinement Fusion under Cooperative Agreement No. DE-FC03-92SF19460, and other agencies.

For questions or comments, contact Valeri N. Goncharov, Editor, Laboratory for Laser Energetics, 250 East River Road, Rochester, NY 14623-1299, (585) 275-5877.

Worldwide-Web Home Page: http://www.lle.rochester.edu/ 


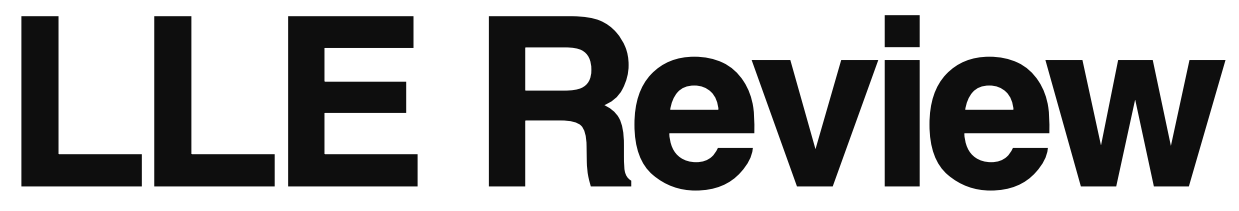

\section{Quarterly Report}

\section{Contents}

In Brief

Performance of 1-THz-Bandwidth, 2-D Smoothing

by Spectral Dispersion and Polarization Smoothing

of High-Power, Solid-State Laser Beams

Effect of Electric Fields on Electron Thermal Transport

in Laser-Produced Plasmas

Effects of Nonuniform Illumination on Implosion Asymmetry

in Direct-Drive Inertial Confinement Fusion

Convective Stimulated Brillouin Scattering (SBS)

in One- and Two-Ion Plasmas

Imprint Efficiency Measurements in Laser-Driven Plastic Foils Using Beams with Different Angles of Incidence

Stopping of Directed Energetic Electrons

in High-Temperature Hydrogenic Plasmas

Operation of a Single-Photon-Counting X-Ray CCD Camera

Spectrometer in a Petawatt Environment

Theory of Laser-Induced Adiabat Shaping in Inertial Fusion Implosions: The Relaxation Method

Using Nuclear Data and Monte Carlo Techniques to Study Areal Density and Mix in $\mathrm{D}_{2}$ Implosions

Publications and Conference Presentations 



\section{In Brief}

This volume of the LLE Review, covering January-March 2004, features "Performance of 1-THzBandwidth, 2-D Smoothing by Spectral Dispersion and Polarization Smoothing of High-Power, SolidState Laser Beams," by S. P. Regan, J. A. Marozas, R. S. Craxton, J. H. Kelly, W. R. Donaldson, P. A. Jaanimagi, D. Jacobs-Perkins, R. L. Keck, T. J. Kessler, D. D. Meyerhofer, T. C. Sangster, W. Seka, V.A. Smalyuk, S. Skupsky, and J. D. Zuegel (p. 49). Laser-beam smoothing achieved with 1-THz-bandwidth, two-dimensional smoothing by spectral dispersion and polarization smoothing on the 60-beam, 30-kJ, 351-nm OMEGA laser system is reported. These beam-smoothing techniques are directly applicable to direct-drive ignition target designs for the 192-beam, 1.8-MJ, 351-nm National Ignition Facility. Equivalent-target-plane images for constant-intensity laser pulses of varying duration were used to determine the smoothing. The properties of the phase plates, frequency modulators, and birefringent wedges were simulated and found to be in good agreement with the measurements.

Additional highlights of research presented in this issue include the following:

- V. N. Goncharov (p. 54) considers the contribution of the gradients in the laser-induced electric field to the current flow, heat flux, and electric stress tensor in laser-produced plasmas. The transport coefficients, previously derived in the limit $Z>>1$, are obtained for an arbitrary ion charge $Z$. It is shown that the ponderomotive terms significantly modify the thermal transport near the laser turning points and the critical surface.

- C. K. Li, F. H. Séguin, J. A. Frenje, R. D. Petrasso-PSFC-MIT, along with J. A. Delettrez, P. W. McKenty, T. C. Sangster, R. L. Keck, J. M. Soures, F. J. Marshall, D. D. Meyerhofer, V. N. Goncharov, J. P. Knauer, P. B. Radha, S. P. Regan, and W. Seka-LLE (p. 67) study the target areal-density $(\rho R)$ asymmetries in OMEGA direct-drive spherical implosions. The rms variation $\langle\delta \rho R\rangle /\langle\rho R\rangle$ for a low-mode-number structure is approximately proportional to the rms variation of on-target laser intensity $\langle\delta I\rangle /\langle I\rangle$ with an amplification factor of $\sim 1 / 2\left(C_{r}-1\right)$, where $C_{r}$ is the capsule convergence ratio. This result has critical implications for future work on the National Ignition Facility (NIF) as well as on OMEGA.

- M. V. Kozlov, C. J. McKinstrie, and A. V. Maximov (p. 73) use the ion-fluid and Poisson (IFP) equations with phenomenological damping terms and the light-wave equation to describe stimulated Brillouin scattering (SBS) in one- and two-ion plasmas. A computer code is tested by comparing numerical and analytical results in the linear limit. The code is used to compare effects of Landau damping, pump depletion, and ion-acoustic nonlinearities on the saturation of SBS in one- and twoion plasmas. In the latter, SBS from fast and slow ion-acoustic waves are considered separately. SBS is simulated for hydrocarbon $(\mathrm{CH})$ plasmas with parameters typical for experiments on OMEGA.

- V. A. Smalyuk, T. R. Boehly, V. N. Goncharov, O. V. Gotchev, J. P. Knauer, D. D. Meyerhofer, and T. C. Sangster (p. 90) report measurements of the imprint efficiency in 20- $\mu$ m-thick plastic foils driven by $351-\mathrm{nm}$ laser light at an intensity $\sim 2 \times 10^{14} \mathrm{~W} / \mathrm{cm}^{2}$. The measured target spatial modulations were imprinted from spatial laser nonuniformities during laser-ablated plasma formation at the beginning of the drive. The laser modulations consisted of broadband nonuniformities from six beams incident at $23^{\circ}$ to the target normal and single-mode perturbations from one beam incident at $48^{\circ}$ to the target normal. The measurements were performed at a spatial wavelength of $60 \mu \mathrm{m}$ with 
and without smoothing by spectral dispersion (SSD). The measured imprint efficiencies at $60-\mu \mathrm{m}$ spatial wavelength were $2.5 \pm 0.2 \mu \mathrm{m}$ for the beam with $48^{\circ}$ angle of incidence and $3.0 \pm 0.3 \mu \mathrm{m}$ for the beams with $23^{\circ}$ angle of incidence. The SSD reduced modulations by a factor of $\sim 2.5$ at the same spatial wavelength.

- C. K. Li and R. D. Petrasso-PSFC-MIT (p.97) present an analytical model of the interaction of directed energetic electrons with a high-temperature hydrogenic plasma. The randomizing effect of scattering off both plasma ions and electrons is treated from a unified point of view. For electron energies of less than $3 \mathrm{MeV}$, electron scattering is equally important. The net effect of multiple scattering is to reduce the penetration from 0.54 to $0.41 \mathrm{~g} / \mathrm{cm}^{2}$ for $1-\mathrm{MeV}$ electrons in a $300-\mathrm{g} / \mathrm{cm}^{3}$ plasma at $5 \mathrm{keV}$. These considerations are relevant to "fast ignition" and to fuel preheat for inertial confinement fusion.

- C. Stoeckl, W. Theobald, and T. C. Sangster; M. H. Key, P. Patel, and B. B. Zhang-LLNL; and R. Clarke, S. Karsch, and P. Norreys-RAL (p. 103) report on shielding strategies to optimize the signalto-background ratio and to obtain high-quality $x$-ray spectra. The use of a single-photon-counting $\mathrm{x}$-ray CCD camera as an $\mathrm{x}$-ray spectrometer is a well-established technique in ultra-short-pulse laser experiments. In the single-photon-counting mode, the pixel value of each readout pixel is proportional to the energy deposited from the incident x-ray photon. For photons below $100 \mathrm{keV}$, a significant fraction of the events deposit all energy in a single pixel. A histogram of the pixel readout values gives a good approximation of the x-ray spectrum. This technique requires almost no alignment, but it is very sensitive to signal-to-background issues, especially in a high-energy petawatt environment.

- R. Betti and K. Anderson (p. 106) present the theory of the adiabat profile induced by a strong shock propagating through a relaxed density profile in inertial confinement fusion (ICF) capsules. The relaxed profile is produced through a laser prepulse, while the adiabat-shaping shock is driven by the foot of the main laser pulse. The adiabat shape is calculated for the cases of intense, short prepulses and weak, long prepulses. The theoretical adiabat profiles accurately reproduce the simulation results to within a few-percent error. ICF capsules with a shaped adiabat are expected to benefit from improved hydrodynamic stability while maintaining the same one-dimensional performances as flatadiabat shells.

- S. S. Kurebayashi, J. A. Frenje, F. H. Séguin, J. R. Rygg, C. K. Li, and R. D. Petrasso-PSFC-MIT; V. Yu. Glebov, J. A. Delettrez, T. C. Sangster, D. D. Meyerhofer, C. Stoeckl, and J. M. Soures-LLE; and P. A. Amendt, S. P. Hatchett, and R. E. Turner-LLNL (p. 122) investigate models for determining the areal density of hot fuel $\left(\rho R_{\text {hot }}\right)$ in compressed, $\mathrm{D}_{2}$-filled capsules. Measurements from three classes of direct-drive implosions on OMEGA were combined with Monte Carlo simulations to assess the impact of mix and other factors on the determination of $\rho R_{\text {hot }}$. The results of the Monte Carlo calculations were compared to predictions of simple commonly used models that use ratios of either secondary $\mathrm{D}^{3} \mathrm{He}$ proton yields or secondary DT neutron yields to primary DD neutron yields to provide estimates of $\rho R_{\mathrm{hot}, p}$ or $\rho R_{\mathrm{hot}, n}$, respectively, for $\rho R_{\mathrm{hot}}$.

Valeri N. Goncharov

Editor 


\section{Performance of 1-THz-Bandwidth, 2-D Smoothing by Spectral Dispersion and Polarization Smoothing of High-Power, Solid-State Laser Beams}

\section{Introduction}

A direct-drive inertial confinement fusion (ICF) implosion of a spherical capsule containing thermonuclear fuel is initiated by the ablation of material from the outer shell surface with overlapped, intense laser beams. ${ }^{1}$ The ablated shell mass forms a coronal plasma that surrounds the target and accelerates the shell inward via the rocket effect. ${ }^{1,2}$ Perturbations at the ablation surface resulting from target imperfections and laser irradiation nonuniformities (known as laser imprint) are amplified by the ablative Rayleigh-Taylor (RT) instability as the shell accelerates inward and are further amplified during the deceleration phase. ${ }^{3-9}$ The RT instability can reduce the thermonuclear yield of the implosion. ${ }^{1,2}$ The direct-drive ICF program strives to reduce laser imprint levels by uniform laser irradiation of the target. High-compression direct-drive experiments require a $1 \% \mathrm{rms}$ level of the on-target laser irradiation nonuniformity averaged over a few hundred picoseconds. ${ }^{10}$ This is accomplished on the 60 -beam, 30-kJ, 351-nm OMEGA laser system ${ }^{11}$ using two-dimensional smoothing by spectral dispersion (2-D SSD),,${ }^{10,12-14}$ distributed phase plates (DPP's), ${ }^{15,16}$ polarization smoothing (PS) utilizing birefringent wedges, ${ }^{17-19}$ and multiple-beam overlap. ${ }^{20}$ These techniques are directly applicable to direct-drive ignition target designs ${ }^{21}$ planned for the 1.8-MJ, 351-nm, 192-beam National Ignition Facility (NIF) at the Lawrence Livermore National Laboratory. ${ }^{22}$

The 2-D SSD UV bandwidth $\left(\Delta v_{\mathrm{UV}}\right)$ on OMEGA was recently increased to $1 \mathrm{THz}$, and polarization smoothing was added through the installation of a birefringent wedge in each of the 60 beams. The amount of smoothing achieved with 1-THz, 2-D SSD and PS is reported. The experimental techniques outlined in Ref. 14 are used to determine the singlebeam irradiation nonuniformity from the measured ultraviolet equivalent-target-plane (UVETP) images of laser pulses having constant intensity and varying duration. Simulations of the experimental data using the properties of the phase plates, frequency modulators, and birefringent wedges are shown to be in good agreement with the measured results.

\section{2-D SSD and PS}

Laser-beam nonuniformities can be significantly reduced for high-power/energy glass lasers using 1-THz, 2-D SSD and PS. Two-dimensional SSD reduces the single-beam irradiation nonuniformity as a function of time, while the PS provides an additional, instantaneous reduction by a factor of $\sqrt{2}$ in the on-target nonuniformity. ${ }^{10}$ The temporal dynamics of the laser-beam smoothing with 2-D SSD and PS are illustrated in Fig. 98.1. The curves plotted in Fig. 98.1 for 0.2-THz, 2-D SSD (dashed line); 1-THz, 2-D SSD (dotted line); and 1-THz,

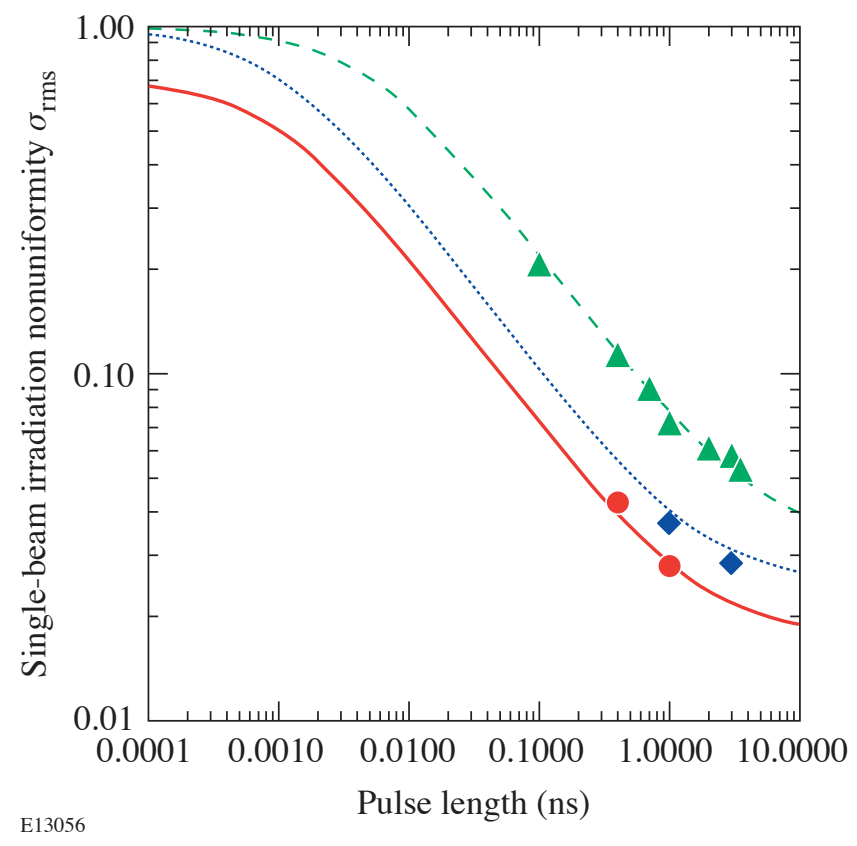

Figure 98.1

The single-beam irradiation nonuniformity $\sigma_{\mathrm{rms}}$ is plotted as a function of time for three laser-beam-smoothing conditions under consideration. Smoothing curves of the predicted $\sigma_{\mathrm{rms}}$ [see Eq. (1)] are plotted for 0.2-THz, 2-D SSD (dashed line); 1-THz, 2-D SSD (dotted line); and 1-THz, 2-D SSD with PS (solid line). The experimental results are plotted for 0.2-THz, 2-D SSD (triangles); 1-THz, 2-D SSD (diamonds); and 1-THz, 2-D SSD with PS (circles). The model shows excellent agreement with the experimental results that are a compilation of data from more than 200 laser shots. The error bars are smaller than the symbols. 
2-D SSD with PS (solid line) represent the model predictions ${ }^{14}$ for the single-beam irradiation nonuniformity $\sigma_{\text {rms }}$ :

$$
\sigma_{\mathrm{rms}}=\sqrt{\sigma_{0}^{2}\left(\frac{t_{c}}{t+t_{c}}\right)+\sigma_{\mathrm{asymp}}^{2}},
$$

where $t_{c}=1 / \Delta v_{\mathrm{UV}}$ is the coherence time, $t$ is the averaging time (i.e., pulse length), $\sigma_{0}$ is the initial laser nonuniformity, and $\sigma_{\text {asymp }}$ is the asymptotic level of smoothing with 2-D SSD and PS taken from Eq. (8) of Ref. 23. The $\sigma_{\text {rms }}$ predicted with Eq. (1) has been shown to be nearly indistinguishable from the $\sigma_{\text {rms }}$ predicted with the time-integrated simulation described below. ${ }^{14}$ The model shows excellent agreement with the experimental results as discussed in the next section. The laserbeam smoothing parameters are listed in Table 98.I. As seen in Fig. 98.1, prior to reaching asymptotic levels, increasing $\Delta v_{\mathrm{UV}}$ of the 2-D SSD from $0.2 \mathrm{THz}$ (dashed line) to $1 \mathrm{THz}$ (dotted line) reduces the amount of time needed to smooth to a given level of nonuniformity by a factor of 5 . Also, for time $t \gg t_{c}$, it reduces the level of nonuniformity at any given time by a factor of $\sqrt{5}$. A comparison of the smoothing curve for the 1-THz, 2-D SSD (dotted line) with the curve for 1-THz, 2-D SSD with PS (solid line) shows that PS provides an instantaneous reduction in $\sigma_{\mathrm{rms}}$ by $\sqrt{2}$.

Table 98:I: Specifications for laser-beam smoothing.

\begin{tabular}{|c|c|c|c|c|}
\hline \hline $\begin{array}{c}\text { 2-D SSD } \\
\Delta v_{\mathrm{UV}}(\mathrm{THz})\end{array}$ & PS & $\begin{array}{c}t_{c}=1 / \Delta v_{\mathrm{UV}} \\
(\mathrm{ps})\end{array}$ & $\sigma_{0}$ & $\sigma_{\text {asymp }}$ \\
\hline 0.2 & off & 5 & 1.00 & $3.30 \times 10^{-2}$ \\
\hline 1.0 & off & 1 & 1.00 & $2.52 \times 10^{-2}$ \\
\hline 1.0 & on & 1 & 0.707 & $1.77 \times 10^{-2}$ \\
\hline \hline
\end{tabular}

Laser-beam smoothing with PS is instantaneous, while 2-D SSD produces uniform far-field spots on target in a timeaveraged sense. One-dimensional SSD is achieved on OMEGA by frequency modulating the phase of the laser beam, wavelength dispersing the beam using a diffraction grating, and passing the beam through a phase plate placed just before the focusing lens. ${ }^{10,12}$ Two-dimensional SSD is achieved by applying the 1-D SSD operations in two orthogonal directions. ${ }^{10}$ Highly reproducible spatial intensity envelopes and speckle distributions are produced in the far field. The implementation of PS on OMEGA is described elsewhere. ${ }^{10}$ Each UV beam, polarized at $45^{\circ}$ to the ordinary and extraordinary axes of a birefringent wedge placed before the phase plate, is split into two beams of equal intensities that refract through the wedged surface at slightly different angles and focus on target with a separation of $\sim 85 \mu \mathrm{m}$, about 37 times the beam's diffraction limited width ( $f$ number times the laser wavelength $=2.3 \mu \mathrm{m}$ ). The two beams each produce essentially the same speckle pattern on target, determined by the phase plate, but since these patterns are spatially uncorrelated with opposite polarization states, they combine through the addition of intensities rather than electric fields. This leads to the instantaneous reduction in the nonuniformity by a factor of $\sqrt{2}$.

The time-integrated far field is calculated by temporally integrating the modulus squared of a 2-D spatial Fourier transform of the UV near field. The complex-valued electric field that describes the UV near field can be written as

$$
\begin{aligned}
\vec{E}(x, y, t) \equiv & E_{0}(x, y, t) \exp \left[i \phi_{2-\mathrm{D} \mathrm{SSD}}(x, y, t)\right] \\
& \cdot \exp \left[i \phi_{B}(x, y, t)\right] \cdot \exp \left[i \phi_{\mathrm{DPP}}(x, y)\right] \\
& \cdot\left\{\hat{x}+\exp \left[i \phi_{\mathrm{PS}}(y)\right] \hat{y}\right\},
\end{aligned}
$$

where $E_{0}(x, y, t)$ defines the temporal and spatial beam envelope, $\phi_{2-\mathrm{D} \mathrm{SSD}}(x, y, t)$ is the 2-D SSD phase contribution, $\phi_{B}(x, y, t)$ is the intensity-dependent phase contribution of the $B$-integral, ${ }^{24} \phi_{\mathrm{DPP}}(x, y)$ is the static phase-plate contribution that depends on the particular phase-plate design, and $\phi_{\mathrm{PS}}(y)$ is a linear phase term due to the birefringent wedge. The ideal spatially and temporally varying phase due to 2 -D SSD can be expressed as

$$
\begin{aligned}
\phi_{2-\mathrm{D} \mathrm{SSD}}(x, y, t) \equiv & 3 \delta_{M_{x}} \sin \left[\omega_{M_{x}}\left(t+\xi_{x} x\right)\right] \\
& +3 \delta_{M_{y}} \sin \left[\omega_{M_{y}}\left(t+\xi_{y} y\right)\right],
\end{aligned}
$$

where the $x$ and $y$ subscripts denote the two smoothing dimensions, $\delta_{M_{x, y}}$ is the modulation depth, $v_{M_{x, y}} \equiv \omega_{M_{x, y}} / 2 \pi$ is the RF modulation frequency, and $\xi_{x, y}$ describes the variation in phase across the beam due to the angular grating dispersion. The factor of 3 in Eq. (3) indicates that the electric field has undergone frequency tripling from the IR to UV. The 2-D SSD system parameters on OMEGA for the UVETP measurements are $\delta_{M_{x}}=14.3, v_{M_{x}} \equiv 10.4 \mathrm{GHz}, \xi_{x}=0.300 \mathrm{~ns} / \mathrm{m}$, $\delta_{M_{y}}=6.15, v_{M_{y}} \equiv 3.30 \mathrm{GHz}$, and $\xi_{y}=1.13 \mathrm{~ns} / \mathrm{m}$, assuming a nominal beam diameter of $27.5 \mathrm{~cm}$. The modulation depths 
are given for the IR. The IR bandwidths, defined as $\Delta v_{x}=2 \delta_{M_{x}} v_{M_{x}}$ and $\Delta v_{y}=2 \delta_{M_{y}} v_{M_{y}}$, correspond to $\Delta \lambda_{x}=$ $11.0 \AA$ and $\Delta \lambda_{y}=1.50 \AA$. The maximum angular spread $\Delta \theta$ is given by $\Delta \theta=\xi(c / \lambda) \Delta \lambda$, where $c$ is the speed of light and $\lambda=1053 \mathrm{~nm}$. $B$-integral effects are negligible for all cases except when the frequency modulation is turned off. ${ }^{14}$ The linear phase term due to the birefringent wedge is

$$
\phi_{\mathrm{PS}}(y)=k_{0} \sin \left(\Delta \theta_{\mathrm{PS}}\right) y,
$$

where $k_{0}=2 \pi / \lambda_{\mathrm{UV}}$ is the UV laser wave number with $\lambda_{\mathrm{UV}}=$ $351 \mathrm{~nm}$ and $\Delta \theta_{\mathrm{PS}}=47 \mu \mathrm{rad}$ is the angular separation due to the birefringent wedge.

\section{Experimental Results and Analysis}

A description of the UVETP diagnostic used in this study can be found in Ref. 14. This diagnostic uses a full-aperture optical wedge in one of the 60 beams to direct a small fraction of the laser light to a phase plate and an OMEGA focusing lens, and it records the UVETP image on a CCD camera (see Fig. 1 of Ref. 14). The capability of the UVETP diagnostic to resolve fully individual speckles has been demonstrated. ${ }^{14}$ Shots with PS have the birefringent wedge placed in the beam before the full-aperture optical wedge. A far-field image recorded with the UVETP diagnostic of a 1-ns square laser pulse with 1-THz, 2-D SSD and PS is presented in Fig. 98.2. The UVETP diagnostic was configured with a phase plate that produced a far-field spot with a super-Gaussian spatial-intensity envelope $\left[I \sim \exp \left(r / r_{0}\right)^{2.3}\right]$. The image, which has been flat fielded, shows a smooth spatial-intensity envelope [see the singlepixel lineout overplotted on the image in Fig. 98.2]. The spatial resolution and overall detector size of the CCD restrict the UVETP measurement to slightly more than one-half of the laser-beam diameter. As seen in Fig. 98.2, the laser beam is centered on the photodetector, and $560 \mu \mathrm{m}$ of the $1010-\mu \mathrm{m}$ (defined as the $95 \%$ enclosed energy contour) laser spot is sampled. Alignment constraints for the compilation of laser shots under consideration restrict the analysis to the central $\sim 410 \mu \mathrm{m}$ of the laser spot.

The amount of smoothing achieved with 1-THz, 2-D SSD and PS is quantified from the power spectrum of the measured UVETP image of Fig. 98.2. The UVETP images are Fourier transformed with a 2-D Hamming filter applied to the data, in order to obtain the power spectrum defined as the azimuthal sum at each spatial frequency of the square of the Fourier amplitudes. Good agreement is observed between the measured power spectrum for the 1-THz, 2-D SSD, and PS presented in Fig. 98.3(a) and the modeled spectrum determined from simulated time-integrated far field. The wave number is defined as $k=2 \pi / \lambda_{\mathrm{SN}}$, where $\lambda_{\mathrm{SN}}$ is the spatial nonuniformity wavelength, and the $\ell$ mode is defined as $\ell=k R$, where $R=500 \mu \mathrm{m}$ is the spherical target radius. The $\sigma_{\mathrm{rms}}$ reaches $2.7 \%$ averaged over $1 \mathrm{~ns}$, in agreement with the predicted $2.8 \%$. The $\sigma_{\mathrm{rms}}$ is defined here as the square root of the ratio of the integral of power in the high frequencies (i.e., $k \geq$ $0.04 \mu \mathrm{m}^{-1}$ in the OMEGA target plane) to the integral of power in the low frequencies (i.e., $k<0.04 \mu \mathrm{m}^{-1}$ ). The envelope and speckle were separated at wave number $0.04 \mu \mathrm{m}^{-1}$ in the calculation of $\sigma_{\mathrm{rms}}$ for two reasons. First, virtually all of the envelope power is contained in the first three terms of the Fourier transform, which have wave numbers $k<0.04 \mu \mathrm{m}^{-1}$; therefore, inclusion of additional terms in this sum increases the envelope power by insignificant amounts. Second, the smallest wave number of nonuniformity that can be smoothed on OMEGA with 1-THz, 2-D SSD falls between the third and fourth terms of the Fourier transform with wave numbers 0.031 and $0.046 \mu \mathrm{m}^{-1}$. Therefore, all the wave numbers that 2-D SSD can smooth are in the range $0.04 \mu \mathrm{m}^{-1} \leq k<k_{c}$, where $k_{c}=2.7 \mu \mathrm{m}^{-1}$ is the cutoff wave number. The birefringent wedge was removed from the beamline, and another UVETP image was recorded to quantify the amount of smoothing

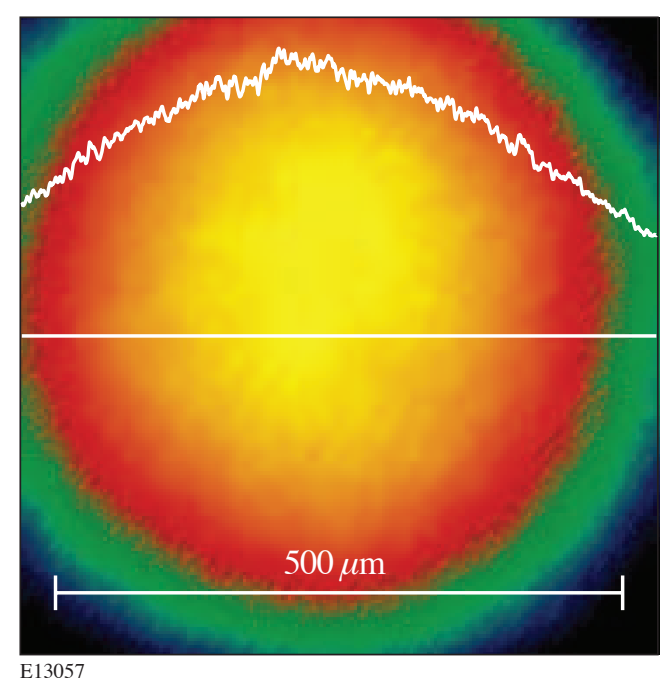

Figure 98.2

Measured UVETP image of a 1-ns square laser pulse with 1-THz, 2-D SSD and PS (shot 22835). As demonstrated with the single-pixel lineout through the center of the beam, the laser beam has a smooth spatial-intensity envelope. The spatial resolution and overall detector size of the CCD restrict the UVETP measurement to slightly more than one-half of the laser-beam profile. The laser beam is centered nominally on the photodetector, and $560 \mu \mathrm{m}$ of the $1010-\mu \mathrm{m}$ laser spot (defined as the $95 \%$ enclosed energy contour) is sampled. 
achieved with 1-THz, 2-D SSD alone (i.e., no PS). Again, good agreement is shown in Fig. 98.3(b) between the measured power spectrum and the simulation with the expected $\sqrt{2}$ increase in $\sigma_{\mathrm{rms}}$ observed.
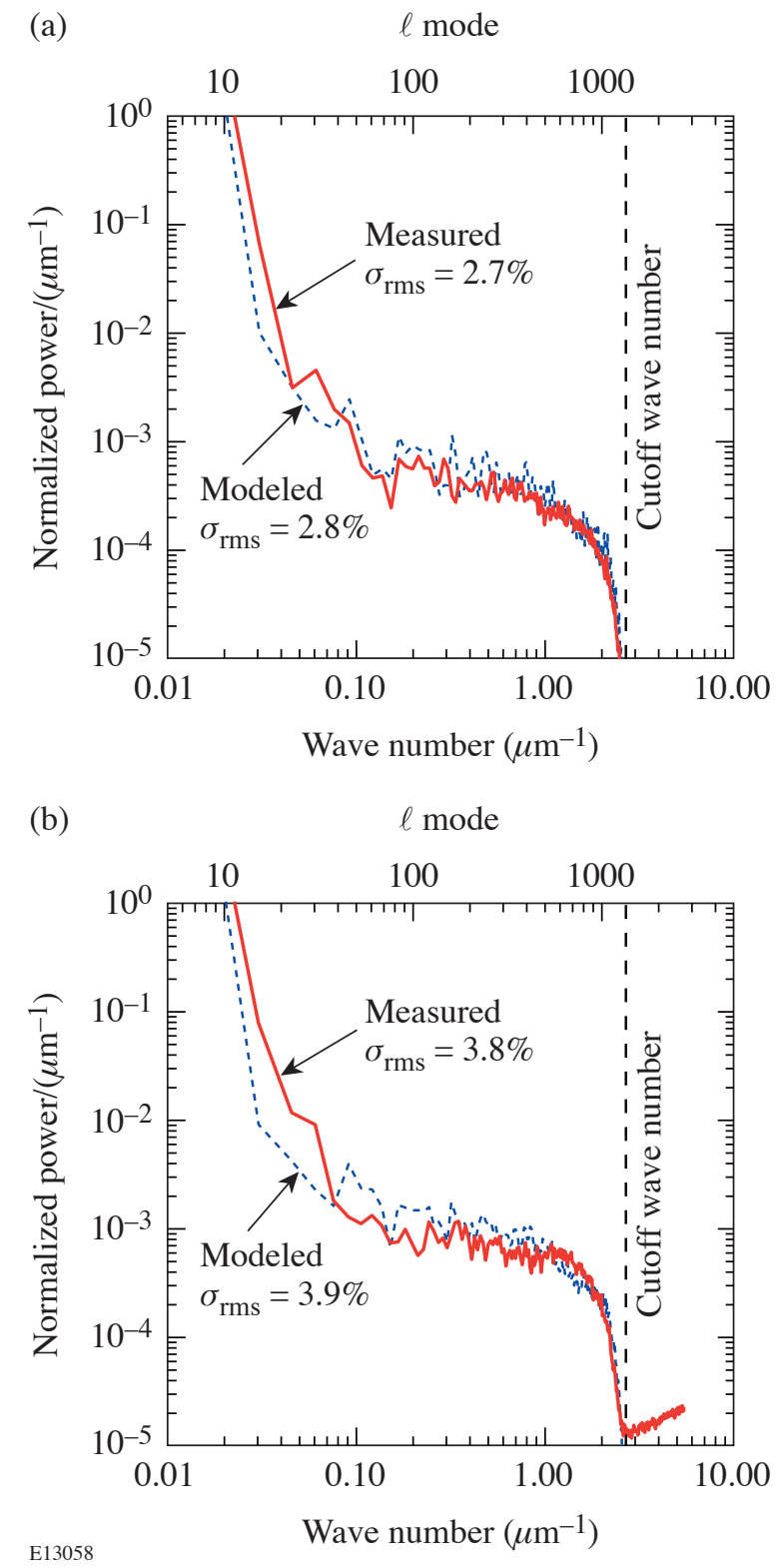

Figure 98.3

Power spectra calculated from UVETP images of (a) 1-ns square laser pulse with 1-THz, 2-D SSD and PS (shot 22835) and (b) 1-ns square laser pulse with 1-THz, 2-D SSD and no PS (shot 18249). The wave number is defined as $k=2 \pi / \lambda_{\mathrm{SN}}$, where $\lambda_{\mathrm{SN}}$ is the spatial nonuniformity wavelength, and the $\ell$ mode is defined as $\ell=k R$, where $R=500 \mu \mathrm{m}$ is the spherical target radius. The solid lines represent the measured power spectra; the dashed lines represent the simulations, which are in agreement with the measured results.
The measured $\sigma_{\text {rms }}$ with 1-THz, 2-D SSD was also examined using 0.4-, 1.0-, and 3.0-ns square laser pulses. As shown in Fig. 98.1, the 1-THz, 2-D SSD model [Eq. (1)] is in agreement with the measured results. A more-extensive study of the smoothing rates for $0.2-\mathrm{THz}, 2-\mathrm{D}$ SSD was reported in Ref. 14, where it was shown with laser pulses having constant peak power and pulse lengths ranging from $100 \mathrm{ps}$ to $3.5 \mathrm{~ns}$, that the reduction in laser-irradiation nonuniformity is dependent on the spatial nonuniformity wavelength. The measured $\sigma_{\text {rms }}$ for $0.2-\mathrm{THz}, 2-\mathrm{D}$ SSD is also plotted in Fig. 98.1 for comparison with the 1-THz, 2-D SSD results. As can be seen in Fig. 98.1 for the time $t \gg t_{c}$, but prior to reaching asymptotic levels, the measured $\sigma_{\mathrm{rms}}$ is reduced by $\sqrt{5}$ when $\Delta v_{\mathrm{UV}}$ is increased from $0.2 \mathrm{THz}$ to $1 \mathrm{THz}$, and it is further reduced by $\sqrt{2}$ with PS. On OMEGA, beam overlap provides an additional $\sqrt{10}$ reduction in the nonuniformity on target; therefore, with perfect energy balance and timing of the laser beams, a $\sigma_{\text {rms }} \sim 1 \%$ can be achieved on target with 1-THz, 2-D SSD and PS in a few hundred picoseconds.

\section{Conclusion}

Direct-drive ICF experiments require a laser system with excellent irradiation uniformity. Laser-beam nonuniformities can be significantly reduced for high-power/energy glass lasers using 1-THz, 2-D SSD and PS. UVETP images of a single OMEGA laser beam were recorded to quantify the singlebeam irradiation nonuniformity. The amount of smoothing achieved with 1-THZ, 2-D SSD and PS was determined by analyzing the power spectra of measured UVETP images of square laser pulses of varying duration. Simulated power spectra are in excellent agreement with the experimental data and permit confident extrapolation to MJ-class laser systems.

\section{ACKNOWLEDGMENT}

The authors appreciate the support of the entire OMEGA engineering staff, especially C. Kellogg, S. Stagnitto, R. Huff, and M. Kamm. This work was supported by the U.S. Department of Energy Office of Inertial Confinement Fusion under Cooperative Agreement No. DE-FC03-92SF19460, the University of Rochester, and the New York State Energy Research and Development Authority. The support of DOE does not constitute an endorsement by DOE of the views expressed in this article.

\section{REFERENCES}

1. S. E. Bodner, D. G. Colombant, J. H. Gardner, R. H. Lehmberg, S. P. Obenschain, L. Phillips, A. J. Schmitt, J. D. Sethian, R. L. McCrory, W. Seka, C. P. Verdon, J. P. Knauer, B. B. Afeyan, and H. T. Powell, Phys. Plasmas 5, 1901 (1998).

2. J. D. Lindl, Inertial Confinement Fusion: The Quest for Ignition and Energy Gain Using Indirect Drive (Springer-Verlag, New York, 1998). 
3. D. K. Bradley, J. A. Delettrez, and C. P. Verdon, Phys. Rev. Lett. 68 , 2774 (1992).

4. J. Delettrez, D. K. Bradley, and C. P. Verdon, Phys. Plasmas 1, 2342 (1994).

5. J. D. Kilkenny, S. G. Glendinning, S. W. Haan, B. A. Hammel, J. D. Lindl, D. Munro, B. A. Remington, S. V. Weber, J. P. Knauer, and C. P. Verdon, Phys. Plasmas 1, 1379 (1994).

6. R. Epstein, J. Appl. Phys. 82, 2123 (1997).

7. V. A. Smalyuk, T. R. Boehly, D. K. Bradley, V. N. Goncharov, J. A. Delettrez, J. P. Knauer, D. D. Meyerhofer, D. Oron, and D. Shvarts, Phys. Rev. Lett. 81, 5342 (1998).

8. V. N. Goncharov, J. P. Knauer, P. W. McKenty, P. B. Radha, T. C. Sangster, S. Skupsky, R. Betti, R. L. McCrory, and D. D. Meyerhofer, Phys. Plasmas 10, 1906 (2003).

9. S. P. Regan, J. A. Delettrez, V. N. Goncharov, F. J. Marshall, J. M. Soures, V. A. Smalyuk, P. B. Radha, B. Yaakobi, R. Epstein, V. Yu. Glebov, P. A. Jaanimagi, D. D. Meyerhofer, T. C. Sangster, W. Seka, S. Skupsky, C. Stoeckl, D. A. Haynes, Jr., J. A. Frenje, C. K. Li, R. D. Petrasso, and F. H. Séguin, "Dependence of Shell Mix on Feedthrough in Direct-Drive Inertial Confinement Fusion," to be published in Physical Review Letters.

10. S. Skupsky and R. S. Craxton, Phys. Plasmas 6, 2157 (1999).

11. T. R. Boehly, D. L. Brown, R. S. Craxton, R. L. Keck, J. P. Knauer, J. H. Kelly, T. J. Kessler, S. A. Kumpan, S. J. Loucks, S. A. Letzring, F. J. Marshall, R. L. McCrory, S. F. B. Morse, W. Seka, J. M. Soures, and C. P. Verdon, Opt. Commun. 133, 495 (1997).

12. S. Skupsky, R. W. Short, T. Kessler, R. S. Craxton, S. Letzring, and J. M. Soures, J. Appl. Phys. 66, 3456 (1989).

13. J. E. Rothenberg, J. Opt. Soc. Am. B 14, 1664 (1997).

14. S. P. Regan, J. A. Marozas, J. H. Kelly, T. R. Boehly, W. R. Donaldson, P. A. Jaanimagi, R. L. Keck, T. J. Kessler, D. D. Meyerhofer, W. Seka, S. Skupsky, and V. A. Smalyuk, J. Opt. Soc. Am. B 17, 1483 (2000).

15. T. J. Kessler, Y. Lin, J. J. Armstrong, and B. Velazquez, in Laser Coherence Control: Technology and Applications, edited by H. T. Powell and T. J. Kessler (SPIE, Bellingham, WA, 1993), Vol. 1870, pp. $95-104$
16. Y. Lin, T. J. Kessler, and G. N. Lawrence, Opt. Lett. 21, 1703 (1996)

17. Y. Kato, unpublished notes from work at LLE, 1984; K. Tsubakimoto et al., Opt. Commun. 91, 9 (1992); K. Tsubakimoto et al., Opt. Commun. 103, 185 (1993).

18. Laboratory for Laser Energetics LLE Review 45, 1, NTIS document No. DOE/DP40200-149 (1990). Copies may be obtained from the National Technical Information Service, Springfield, VA 22161; T. E. Gunderman, J.-C. Lee, T. J. Kessler, S. D. Jacobs, D. J. Smith, and S. Skupsky, in Conference on Lasers and Electro-Optics, Vol. 7, 1990 OSA Technical Digest Series (Optical Society of America, Washington, DC, 1990), p. 354

19. T. R. Boehly, V. A. Smalyuk, D. D. Meyerhofer, J. P. Knauer, D. K. Bradley, R. S. Craxton, M. J. Guardalben, S. Skupsky, and T. J. Kessler, J. Appl. Phys. 85, 3444 (1999).

20. F. J. Marshall, J. A. Delettrez, R. Epstein, R. Forties, R. L. Keck, J. H. Kelly, P. W. McKenty, S. P. Regan, and L. J. Waxer, Phys. Plasmas 11 251 (2004).

21. P. W. McKenty, T. C. Sangster, M. Alexander, R. Betti, R. S. Craxton, J. A. Delettrez, L. Elasky, R. Epstein, A. Frank, V. Yu. Glebov, V. N. Goncharov, D. R. Harding, S. Jin, J. P. Knauer, R. L. Keck, S. J. Loucks, L. D. Lund, R. L. McCrory, F. J. Marshall, D. D. Meyerhofer, S. P. Regan, P. B. Radha, S. Roberts, W. Seka, S. Skupsky, V. A. Smalyuk, J. M. Soures, K. A. Thorp, M. Wozniak, J. A. Frenje, C. K. Li, R. D. Petrasso, F. H. Séguin, K. A. Fletcher, S. Padalino, C. Freeman, N. Izumi, J. A. Koch, R. A. Lerche, M. J. Moran, T. W. Phillips, G. J. Schmid, and C. Sorce, "Direct-Drive Cryogenic Target Implosion Performance on OMEGA," to be published in Physics of Plasmas.

22. J. Paisner et al., Laser Focus World 30, 75 (1994).

23. J. E. Rothenberg, in Solid State Lasers for Application to Inertial Confinement Fusion, edited by W. F. Krupke (SPIE, Bellingham, WA, 1995), Vol. 2633, pp. 634-644.

24. J. A. Marozas, S. P. Regan, J. H. Kelly, D. D. Meyerhofer, W. Seka, and S. Skupsky, J. Opt. Soc. Am. B 19, 7 (2002). 


\section{Effect of Electric Fields on Electron Thermal Transport in Laser-Produced Plasmas}

\section{Introduction}

Thermal transport plays an important role in direct-drive inertial confinement fusion. The Spitzer-Härm heat flux ${ }^{1}$ $\mathbf{q}_{\mathrm{SH}}=-\kappa_{\mathrm{SH}} \nabla T$ has been conventionally used in the directdrive inertial confinement fusion (ICF) hydrocodes. Here, $\kappa_{\mathrm{SH}}$ is the Spitzer heat conductivity and $T$ is the electron temperature. In the regions of the steep temperature gradients where $\mathbf{q}_{\mathrm{SH}}$ exceeds a fraction $f$ of the free-stream limit $q_{\mathrm{FS}}=n T v_{T}$, the Spitzer flux is replaced ${ }^{2}$ by $f q_{\mathrm{FS}}$, where $n$ is the electron density, $v_{T}=\sqrt{T / m}$ is the electron thermal velocity, and $f=$ $0.05-0.1$ is the flux limiter. It has been known for more than two decades $^{3-5}$ that, in addition to the terms proportional to the temperature gradients (thermal terms), the heat flux in laserproduced plasmas contains ponderomotive terms that are due to the gradients in the laser electric field. To our best knowledge, no systematic analysis has been performed to address the effect of such terms on the hydrodynamic flow in ICF plasmas. As shown later, the ratio of the ponderomotive terms to the thermal terms is proportional to $R=\alpha\left(v_{E} / v_{T}\right)^{2}\left(L_{T} / L_{E}\right)$, where $v_{E}=e E / m \omega_{L}$ is the electron quiver velocity, $e$ is the electric charge, $E$ is the amplitude of the electric field, $m$ is the electron mass, $\omega_{L}$ is the laser frequency, $L_{T}$ and $L_{E}$ are the temperature and the electric field scale length, and $\alpha$ is a constant. The ratio of the electron quiver velocity to the thermal velocity is small for typical plasma parameters. Indeed, $\left(v_{E} / v_{T}\right)^{2} \simeq 0.4 I_{15} \lambda_{\mu \mathrm{m}}^{2} / T_{\mathrm{keV}}$, where $I_{15}$ is the laser intensity in $10^{15} \mathrm{~W} / \mathrm{cm}^{2}, \lambda_{\mu \mathrm{m}}$ is the laser wavelength in microns, and $T_{\mathrm{keV}}$ is the electron temperature in $\mathrm{keV}$. Using $I_{15} \sim 1$ and $T \sim 2 \mathrm{keV}$, we obtain $\left(v_{E} / v_{T}\right)^{2} \sim 0.02$ for $\lambda_{\mu \mathrm{m}}=$ $0.353 \mu \mathrm{m}$. The ratio $R$, however, can be of the order of unity due to a large ratio $L_{T} / L_{E}$. Indeed, as the laser reaches the turning point where the electron density equals $n_{c} \cos ^{2} \theta$, the electric field decays toward the overdense portion of the shell as $^{6} E \sim E_{\max } \exp \left(-2 / 3 \xi^{3 / 2}\right)$, where $n_{c}=m_{e} \omega_{L}^{2} / 4 \pi e^{2}$ is the critical density, $\theta$ is the laser incidence angle, $\zeta=\left(\omega_{L} L_{n} / c\right)^{2 / 3} z / L_{n}, L_{n} \sim L_{T}$ is the electron-density scale length, and $z$ is the coordinate along the density gradient. Therefore, the electric-field scale length near the turning point becomes $L_{E} \sim L_{T} /\left(\omega_{L} L_{T} / c\right)^{2 / 3}$. Substituting this estimate to the ratio $R$ and using $L_{T} \sim 10 \mu \mathrm{m}$ and $\left(v_{E} / v_{T}\right)^{2} \sim 0.02$ gives
$R \sim \alpha\left(v_{E} / v_{T}\right)^{2}\left(\omega_{L} L_{T} / c\right)^{2 / 3} \sim 0.6 \alpha$. As will be shown later, the coefficient $\alpha$ is numerically large and proportional to the ion charge $Z$; this makes $R$ larger than 1 . This simple estimate shows that the ponderomotive terms become comparable to the thermal terms in the electron thermal flux near the turning point. In addition, the $p$-polarization of the electric field (polarization that has a field component directed along the density gradient) tunnels through the overdense portion of the shell and gives a resonance electric field at the critical surface. ${ }^{6}$ The gradient of such a field is proportional to the ratio $\omega_{L} / v_{\mathrm{ei}}$, where $v_{\mathrm{ei}}$ is the electron-ion collision frequency at the critical surface. Substituting typical direct-drive experiment parameters into an expression for the electron-ion collision frequency at the critical surface, $v_{\mathrm{ei}} / \omega_{L} \simeq 1.5 \times 10^{-3} \mathrm{Z} / T_{\mathrm{keV}}^{3 / 2}$, shows a significant contribution of the ponderomotive terms to the heat flux near the critical surface.

In this article, the ponderomotive transport coefficients are derived. Such coefficients have been considered previously. ${ }^{3-5,7-9}$ Reference 7 developed a method of solving the kinetic equation by separation of the electron distribution function on the high-frequency component due to the laser field and the low-frequency component of the time-averaged plasma response. Using such a method, the laser fields' contribution to the electron stress tensor was obtained. A similar method was used in Ref. 3, where the importance of the ponderomotive effects on the electron thermal conduction was emphasized. P. Mora and R. Pellat ${ }^{4}$ and I. P. Shkarofsky ${ }^{5}$ have evaluated the contributions of the laser fields into the heat and momentum fluxes. As was pointed out in Ref. 8, by not, however, consistently taking into account the contribution of the electron-electron collisions - the transport coefficients in their results contain wrong numerical factors. A consistent analysis was performed in Ref. 8, where results were obtained in the limit of large ion charge. Such a limit was relaxed in Ref. 9. The latter reference, however, contains numerous typographical errors, so the results will therefore be rederived in this article. The effect of ponderomotive terms on the hydrodynamic flow in direct-drive ICF experiments will be discussed in detail in a forthcoming publication. 


\section{Model}

We consider a fully ionized plasma in a high-frequency electromagnetic field:

$$
\begin{aligned}
& \varepsilon=\frac{1}{2}\left[\mathbf{E}(r, t) e^{-i \omega_{L} t}+\mathbf{E}(r, t)^{*} e^{i \omega_{L} t}\right], \\
& \beta=\frac{1}{2}\left[\mathbf{B}(r, t) e^{-i \omega_{L} t}+\mathbf{B}(r, t)^{*} e^{i \omega_{L} t}\right],
\end{aligned}
$$

where $\mathbf{E}$ and $\mathbf{B}$ are slowly varying (with respect to $e^{i \omega_{L} t}$ ) electric and magnetic fields and $\mathbf{E}^{*}$ and $\mathbf{B}^{*}$ are the complex conjugate (c.c.) of $\mathbf{E}$ and $\mathbf{B}$. The electron distribution function $f$ obeys the Boltzmann equation

$$
\begin{aligned}
& \partial_{t} f+\mathbf{v} \partial_{\mathbf{r}} f+e\left(\mathbf{E}_{0}+\varepsilon+\frac{\mathbf{v} \times \beta}{c}\right) \partial_{\mathbf{p}} f \\
& =J_{\mathrm{ee}}[f, f]+J_{\mathrm{ei}}[f],
\end{aligned}
$$

where $\mathbf{E}_{0}$ is the low-frequency electric field. Here,

$$
J_{\mathrm{ei}}[f]=3 \sqrt{\frac{\pi}{8}}\left(\frac{v_{T}}{v}\right)^{3} v_{\mathrm{ei}} \frac{\partial}{\partial v_{k}}\left[\left(v^{2} \delta_{k j}-v_{k} v_{j}\right) \frac{\partial f}{\partial v_{j}}\right]
$$

is the ion-electron collision operator,

$$
v_{\mathrm{ei}}=\frac{4 \sqrt{2 \pi} e^{4} n \bar{Z} \Lambda}{3 m^{2} v_{T}^{3}}
$$

is the electron-ion collision frequency,

$$
\bar{Z}=\sum_{i} e_{i}^{2} n_{i} / \sum_{i} e_{i} n_{i}
$$

is the average ion charge, $n_{i}$ is the ion number density, $n$ is the electron density, $e_{i}$ is the ion charge, $m$ is the electron mass, $\Lambda$ is the Coulomb logarithm, $v_{T}=\sqrt{T / m}$, and $T$ is the electron temperature. The sum in $\bar{Z}$ is taken over all ion species in the plasma. The electron-electron collision integral is taken in Landau form

$$
\begin{aligned}
& J_{\mathrm{ee}}[f, f]=\frac{2 \pi e^{4} \Lambda}{m^{2}} \\
& \times \frac{\partial}{\partial v_{k}} \int d \mathbf{v}^{\prime} \frac{\left(\mathbf{v}-\mathbf{v}^{\prime}\right)^{2} \delta_{k j}-\left(v-v^{\prime}\right)_{k}\left(v-v^{\prime}\right)_{j}}{\left|\mathbf{v}-\mathbf{v}^{\prime}\right|^{3}} \\
& \times\left(\frac{\partial}{\partial v_{j}}-\frac{\partial}{\partial v_{j}^{\prime}}\right) f(\mathbf{v}) f\left(\mathbf{v}^{\prime}\right) .
\end{aligned}
$$

Next, following Ref. 7, we separate the electron distribution function on the slowly varying part $f_{0}$ and the high-frequency component $f_{1}$ :

$$
f=f_{0}+\frac{1}{2}\left(f_{1} e^{-i \omega_{L} t}+f_{1}^{*} e^{i \omega_{L} t}\right) .
$$

Substituting Eqs. (1), (2), and (8) into Eq. (3) and collecting the terms with equal powers of $e^{i \omega_{L} t}$, we obtain

$$
\begin{aligned}
& \partial_{t} f_{1}-i \omega_{L} f_{1}+\mathbf{v} \partial_{\mathbf{r}} f_{1}+e \mathbf{E}_{0} \partial_{\mathbf{p}} f_{1} \\
& +e \mathbf{E} \partial_{\mathbf{p}} f_{0}-\frac{i e}{\omega_{L}}[\mathbf{v} \times(\nabla \times \mathbf{E})] \partial_{\mathbf{p}} f_{0}
\end{aligned}
$$

$$
=J_{\mathrm{ee}}\left[f_{0}, f_{1}\right]+J_{\mathrm{ee}}\left[f_{1}, f_{0}\right]+J_{\mathrm{ei}}\left[f_{1}\right]
$$

$$
\partial_{t} f_{0}+\mathbf{v} \partial_{\mathbf{r}} f_{0}+e \mathbf{E}_{0} \partial_{\mathbf{p}} f_{0}-J_{\mathrm{ei}}\left[f_{0}\right]-J_{\mathrm{ee}}\left[f_{0}, f_{0}\right]
$$

$$
\begin{aligned}
=\{ & -\frac{e}{4} \mathbf{E} \partial_{\mathbf{p}} f_{1}^{*}+\frac{i e}{4 \omega_{L}}[\mathbf{v} \times(\nabla \times \mathbf{E})] \partial_{\mathbf{p}} f_{1}^{*} \\
& \left.+\frac{1}{4} J_{\mathrm{ee}}\left[f_{1}, f_{1}^{*}\right]+\text { c.c. }\right\} .
\end{aligned}
$$

Then, to relate $f_{1}$ with $f_{0}$, we assume that the laser frequency is high enough so $f_{1}$ can be expanded in series of 
$\omega_{L}^{-1}: f_{1}=f_{1}^{(1)}+f_{1}^{(2)}+\cdots$, where $f_{1}^{(2)} / f_{1}^{(1)} \sim v_{\mathrm{ei}} / \omega_{L}<<1$. Substituting the latter expansion into Eq. (9) gives

$$
\begin{gathered}
f_{1}^{(1)}=-\frac{i e}{\omega_{L}} \mathbf{E} \partial_{\mathbf{p}} f_{0}, \\
f_{1}^{(2)}=-\frac{e}{\omega_{L}^{2}}\left\{\left(\partial_{t}+\mathbf{v} \partial_{\mathbf{r}}\right) \mathbf{E} \partial_{\mathbf{p}} f_{0}-J_{\mathrm{ei}}\left[\mathbf{E} \partial_{\mathbf{p}} f_{0}\right]\right\} .
\end{gathered}
$$

To eliminate $f_{1}$ from Eq. (10) for the low-frequency component of the distribution function, we substitute Eqs. (11) and (12) into Eq. (10). The result takes the form ${ }^{8}$

$$
\begin{aligned}
& \partial_{t} f_{0}+\mathbf{v} \partial_{\mathbf{r}} f_{0}+e \mathbf{E}_{0} \partial_{\mathbf{p}} f_{0}-J_{\mathrm{ei}}\left[f_{0}\right]-J_{\mathrm{ee}}\left[f_{0}, f_{0}\right] \\
& =\frac{e^{2}}{4 \omega_{L}^{2}}\left\{\frac{\nabla|\mathbf{E}|^{2}}{m} \partial_{\mathbf{p}} f_{0}+\frac{1}{2} \frac{\partial^{2} f_{0}}{\partial p_{i} \partial p_{j}}\left(\partial_{t}+\mathbf{v} \partial_{\mathbf{r}}\right)\left(E_{i} E_{j}^{*}+\text { c.c. }\right)\right. \\
& +\left(E_{i} E_{j}^{*}+\text { c.c. }\right)\left[\frac{1}{m} \frac{\partial^{2} f_{0}}{\partial r_{i} \partial p_{j}}+\left(\partial_{t}+\mathbf{v} \partial_{\mathbf{r}}\right) \frac{\partial^{2} f_{0}}{\partial p_{i} \partial p_{j}}\right. \\
& \left.\left.\quad-\partial_{p_{i}} J_{\mathrm{ei}}\left(\partial_{p_{j}} f_{0}\right)+J_{\mathrm{ee}}\left(\partial_{p_{i}} f_{0}, \partial_{p_{j}} f_{0}\right)\right]\right\}
\end{aligned}
$$

Equation (13) is solved assuming a small deviation of the electron distribution function $f_{0}$ from Maxwellian $f_{\mathrm{M}}$ :

$$
f_{0}=f_{\mathrm{M}}[1+\psi(\mathbf{v}, \mathbf{p}, t)]
$$

where $|\psi|<<1$. The kinetic equation for $\psi$ is obtained by substituting the expansion (14) into Eq. (13) and replacing the time derivatives $\partial_{t} f_{\mathrm{M}}$ using the transport conservation equations. These equations, according to the standard procedure, ${ }^{10}$ are obtained by multiplying the kinetic equations by $\left(\mathbf{v}-\mathbf{v}_{0}\right)^{k}$ with $k=0,1,2, \ldots$ and integrating the latter in the velocity space. Here,

$$
\mathbf{v}_{0}=\frac{1}{\rho}\left(\int d \mathbf{v} m \mathbf{v} f_{0}+\int d \mathbf{v}_{i} m_{i} \mathbf{v}_{i} f_{i}\right)
$$

is the mass velocity, $\rho=n m+n_{i} m_{i} \simeq n_{i} m_{i}$ is the mass density, $m_{i}$ is the ion mass, $\mathbf{v}_{i}$ is the ion velocity, and $f_{i}$ is the ion distribution function. When $k=0$, the described procedure yields the mass conservation equation; $k=1$ and $k=2$ give the momentum and energy conservation equations, respectively. Omitting lengthy algebraic manipulations we report the final result: ${ }^{8}$

$$
\partial_{t} n+\nabla\left(n \mathbf{v}_{0}\right)+\nabla(n \mathbf{V})=0
$$

$$
\begin{gathered}
\rho \partial_{t} v_{0 k}+\rho\left(\mathbf{v}_{0} \partial_{\mathbf{r}}\right) v_{0 k}=-\partial_{r_{k}}\left(p_{e}+p_{i}\right)-\partial_{r_{j}} \sigma_{k j}+\rho_{e} E_{0 k} \\
-\frac{e^{2}}{4 m \omega_{L}^{2}}\left\{n_{e} \partial_{r_{k}}|E|^{2}-\partial_{r_{j}}\left[n\left(E_{k} E_{j}^{*}+\text { c.c. }\right)\right]\right\}, \\
\left(\partial_{t}+\mathbf{v}_{0} \partial_{\mathbf{r}}\right)\left(T+\frac{e^{2}|E|^{2}}{\left.6 m \omega_{L}^{2}\right)}-\frac{T}{n} \nabla(n \mathbf{V})+\frac{2}{3 n_{e}} \nabla \mathbf{q}+\frac{2 p_{e}}{3 n} \nabla_{0}\right. \\
=\frac{m v_{E}^{2} v_{\mathrm{ei}}}{3}+\frac{2 e}{3} \mathbf{E}_{0} \mathbf{V}-\frac{2 m}{m_{i}} v_{\mathrm{ei}}\left(T-T_{i}\right) .
\end{gathered}
$$

Here we use the standard definitions

$$
n=\int d \mathbf{v} f_{0}, \quad n \mathbf{V}=\int d \mathbf{v}\left(\mathbf{v}-\mathbf{v}_{0}\right) f_{0}=\mathbf{j} / e,
$$

$$
\mathbf{q}=\frac{m}{2} \int d \mathbf{v}\left(\mathbf{v}-\mathbf{v}_{0}\right)\left(\mathbf{v}-\mathbf{v}_{0}\right)^{2} f_{0}
$$

$$
\sigma_{k j}=m \int d \mathbf{v}\left(v-v_{0}\right)_{k}\left(v-v_{0}\right)_{j} f_{0}-p_{e} \delta_{k j}
$$

where $T_{i}$ is the ion temperature, $n$ is the electron density, $\mathbf{j}$ is the current density, $\mathbf{q}$ is the heat flux, $\sigma_{k j}$ is the stress tensor, $p_{e}$ and $p_{i}$ are the electron and ion pressures, $v_{E}=e E / m \omega_{L}$, and $\rho_{e}=e n+e_{i} n_{i}$ is the charge density. To simplify the derivation of the transport coefficients, we assume $\mathbf{v}_{0}=0$ and neglect terms of the order of $m / m_{i}$. Next, the equation for the correction $\psi$ to the Maxwellian distribution function is derived by substituting Eq. (14) into Eq. (13) and using the conservation equations (16)-(18). The resulting equation takes the form ${ }^{8}$ 


$$
\begin{aligned}
& \partial_{t} \psi+\mathbf{v} \partial_{\mathbf{r}} \psi-\delta J_{\mathrm{ei}}[\psi]-\delta J_{\mathrm{ee}}[\psi] \\
& =\left(\frac{3}{2}-x+\frac{3}{4} \sqrt{\frac{\pi}{x}}\right) \frac{v_{\mathrm{ei}}}{3} \frac{v_{E}^{2}}{v_{T}^{2}}+\left(x-\frac{3}{2}\right) \frac{2 \nabla \mathbf{q}}{3 n T}+\left(\frac{5}{2}-x\right) \frac{\nabla(n \mathbf{V})}{n} \\
& +\mathbf{v}\left[\left(\frac{5}{2}-x\right) \nabla \ln T-\nabla \ln n T+\frac{e \mathbf{E}_{0}}{T}+\left(\frac{x}{3}-1\right) \frac{\nabla v_{E}^{2}}{2 v_{T}^{2}}\right] \\
& +\frac{x}{10} v_{k} \frac{\partial_{r_{j}}\left(\mathbf{v}_{E}^{2}\right)_{j k}}{v_{T}^{2}}+\frac{3 v_{\mathrm{ei}}}{8 x^{3 / 2}} \frac{\left(\mathbf{v}^{2}\right)_{j k}\left(\mathbf{v}_{E}^{2}\right)_{j k}}{v_{T}^{4}} \\
& \quad \times\left[\frac{\sqrt{\pi}}{2}\left(1+\frac{3}{2 x}\right)+\frac{3}{\bar{Z}} \int_{0}^{x} d x^{\prime} e^{-x^{\prime}} \sqrt{x^{\prime}}\left(1-\frac{x^{\prime}}{x}\right)\right] \\
& \quad+\frac{\left(\mathbf{v}^{3}\right)_{j k l}}{8 v_{T}^{4}} \partial_{r_{l}}\left(\mathbf{v}_{E}^{2}\right)_{j k},
\end{aligned}
$$

where $x=v^{2} /\left(2 v_{T}^{2}\right)$,

$$
\begin{aligned}
& \delta J_{\mathrm{ei}}[\psi]= \frac{3 \sqrt{\pi}}{8 x^{3 / 2}} v_{\mathrm{ei}} \partial_{v_{k}}\left[\left(v^{2} \delta_{k j}-v_{k} v_{j}\right) \partial_{v_{j}} \psi\right], \\
& \delta J_{\mathrm{ee}}[\psi]= \frac{3 \sqrt{2 \pi} v_{\mathrm{ei}} v_{T}^{3}}{4 \bar{Z} n_{e} f_{\mathrm{M}}} \partial_{v_{k}}\left\{f_{\mathrm{M}} \int d \mathbf{v}^{\prime} f_{\mathrm{M}}\left(v^{\prime}\right)\right. \\
& \times \frac{\left(\mathbf{v}-\mathbf{v}^{\prime}\right)^{2} \delta_{k j}-\left(v-v^{\prime}\right)_{k}\left(v-v^{\prime}\right)_{j}}{\left|\mathbf{v}-\mathbf{v}^{\prime}\right|^{3}} \\
&\left.\times\left[\partial_{v_{j}} \psi(\mathbf{v})-\partial_{v_{j}^{\prime}} \psi\left(\mathbf{v}^{\prime}\right)\right]\right\}, \\
&\left(\mathbf{v}^{2}\right)_{j k} \equiv v_{j} v_{k}-\frac{v^{2}}{3} \delta_{j k}, \\
&\left(\mathbf{v}^{3}\right)_{j k l} \equiv v_{j} v_{k} v_{l}-\frac{v^{2}}{5}\left(v_{j} \delta_{k l}+v_{k} \delta_{j l}+v_{l} \delta_{j k}\right),
\end{aligned}
$$




$$
\begin{gathered}
\mathbf{j}^{(1)}=e n v_{T} \lambda_{e}\left[\alpha_{j 1}^{T} \nabla \ln T+\alpha_{j 2}^{T}\left(\nabla \ln n T-\frac{e \mathbf{E}_{0}}{T}\right)\right] \\
\mathbf{q}^{(1)}=n T v_{T} \lambda_{e}\left[\alpha_{q 1}^{T} \nabla \ln T+\alpha_{q 2}^{T}\left(\nabla \ln n T-\frac{e \mathbf{E}_{0}}{T}\right)\right] \\
\sigma_{k j}^{(1)}=\frac{4}{15} \alpha_{\sigma}^{E} m n\left(\mathbf{v}_{E}^{2}\right)_{k j}
\end{gathered}
$$

where $\lambda_{e}=v_{T} / \nu_{\mathrm{ei}}$ is the electron mean-free path. The numerical coefficients in Eqs. (29)-(31) have the forms

$$
\begin{aligned}
\alpha_{j 1(j 2)}^{T} & =\frac{4}{3 \sqrt{\pi}} \int_{0}^{\infty} d x x^{3 / 2} e^{-x} \Phi_{13(14)}(x), \\
\alpha_{q 1(q 2)}^{T} & =\frac{4}{3 \sqrt{\pi}} \int_{0}^{\infty} d x x^{5 / 2} e^{-x} \Phi_{13(14)}(x), \\
\alpha_{\sigma}^{E} & =\frac{4}{\sqrt{\pi}} \int_{0}^{\infty} d x x^{5 / 2} e^{-x} \Phi_{11}(x) .
\end{aligned}
$$

Equations (29)-(31) show that the electric current and the heat flux in the first approximation are proportional to the gradients in temperature and pressure. ${ }^{1,10,11}$ The stress tensor, on the other hand, depends on the laser electric field. ${ }^{3,7}$ Even though the functions $\Phi_{11}$ and $\Phi_{22}$ do not enter into the first-order heat flux, they contribute to the heat flux in the second approximation. Thus, we need to find all four functions $\Phi_{11-14}$. The general form of the solution $\psi_{1}$ [Eq. (28)] can be separated on the following three types of functions: type I depends only on the velocity modulus $\psi_{1}^{(\mathrm{I})}=\Phi(x)$; type II is proportional to the velocity vector and velocity modulus $\psi_{1}^{(\mathrm{II})}=A_{j} v_{j} \Phi(x)$; and type III depends on the velocity tensor and velocity modulus $\psi_{1}^{(\mathrm{III})}=\left(\mathbf{v}^{2}\right)_{i j}\left(\mathbf{v}_{E}^{2}\right)_{i j} \Phi(x)$, where $A_{i}$ is the vector proportional to the temperature, pressure gradients, or the electric field $\mathbf{E}_{0}$. According to such a classification, the governing equations for the functions of each type become

$$
\text { Type I: } \delta J_{\mathrm{ee}}[\Phi(x)]=\phi(x)
$$

Type II: $A_{j}\left\{\Phi(x) \delta J_{\mathrm{ei}}\left[v_{j}\right]+\delta J_{\mathrm{ee}}\left[v_{j} \Phi(x)\right]\right\}=\phi(x) \mathbf{v A}$

$$
\begin{aligned}
\text { Type III: } & \left(\mathbf{v}_{E}^{2}\right)_{i j}\left\{\Phi(x) \delta J_{\mathrm{ei}}\left[\left(\mathbf{v}^{2}\right)_{i j}\right]\right. \\
& \left.+\delta J_{\mathrm{ee}}\left[\left(\mathbf{v}^{2}\right)_{i j} \Phi(x)\right]\right\}=\phi(x)\left(\mathbf{v}^{2}\right)_{i j}\left(\mathbf{v}_{E}^{2}\right)_{i j},
\end{aligned}
$$

where $\phi(x)$ is defined by the right-hand side of Eq. (27). Since the ion-electron collision operator has a very simple form, it is straightforward to calculate $J_{\mathrm{ei}}\left[v_{j}\right]$ and $J_{\mathrm{ei}}\left[\left(\mathbf{v}^{2}\right)_{i j}\right]$ using Eq. (23):

$$
\begin{gathered}
\delta J_{\mathrm{ei}}\left[v_{j}\right]=-\frac{3 \sqrt{\pi}}{4 x^{3 / 2}} v_{\mathrm{ei}} v_{j}, \\
\delta J_{\mathrm{ei}}\left[\left(v^{2}\right)_{i j}\right]=-\frac{9 \sqrt{\pi}}{4 x^{3 / 2}} v_{\mathrm{ei}}\left(\mathbf{v}^{2}\right)_{i j} .
\end{gathered}
$$

The electron-electron operator is more complicated, and the evaluation of $\delta J_{\mathrm{ee}}[\Phi(x)], \delta J_{\mathrm{ee}}\left[v_{j} \Phi(x)\right]$, and $\delta J_{\mathrm{ee}}\left[\left(\mathbf{v}^{2}\right)_{i j} \Phi\right]$ requires lengthy algebra. Below is a detailed calculation of $\delta J_{\text {ee }}[\Phi(x)]$. The integral part in the electron-electron collision operator can be rewritten as

$$
\begin{aligned}
& \int d \mathbf{v}^{\prime} f_{\mathrm{M}}\left(v^{\prime}\right) \frac{\left(\mathbf{v}-\mathbf{v}^{\prime}\right)^{2} \delta_{k j}-\left(v-v^{\prime}\right)_{k}\left(v-v^{\prime}\right)_{j}}{\left|\mathbf{v}-\mathbf{v}^{\prime}\right|^{3}} \\
& \times\left[\frac{v_{j}}{v_{T}^{2}} \Phi^{\prime}(x)-\frac{v_{j}^{\prime}}{v_{T}^{2}} \Phi^{\prime}\left(x^{\prime}\right)\right]=\sum(x) v_{k},
\end{aligned}
$$

where function $\Sigma(v)$ is found by multiplying Eq. (40) by $v_{k}$. This yields

$$
\begin{aligned}
& 2 \pi \int d v^{\prime} \frac{v^{\prime 2}}{v_{T}^{2}} f_{\mathrm{M}}\left(v^{\prime}\right) v^{2} v^{\prime 2}\left[\Phi^{\prime}(x)-\Phi^{\prime}\left(x^{\prime}\right)\right] \\
& \quad \times \int_{-1}^{1} d y \frac{1-y^{2}}{\left(v^{2}+v^{\prime 2}-2 v v^{\prime} y\right)^{3 / 2}}=\sum(x) v^{2},
\end{aligned}
$$


where $y=\cos \theta$ and $\theta$ is the angle between $\mathbf{v}$ and $\mathbf{v}^{\prime}$. Integration over the angles gives

$$
\begin{aligned}
& \int_{-1}^{1} d y \frac{1-y^{2}}{\left(v^{2}+v^{\prime 2}-2 v v^{\prime} y\right)^{3 / 2}} \\
& =\frac{2}{3 v^{\prime 3} v^{3}}\left[v^{3}+v^{\prime 3}-\left(v^{2}+v v^{\prime}+v^{\prime 2}\right)\left|v-v^{\prime}\right|\right] \\
& =\left\{\begin{array}{ll}
4 /\left(3 v^{3}\right) & \text { if } v^{\prime}<v \\
4 /\left(3 v^{\prime 3}\right) & \text { if } v^{\prime}>v
\end{array} .\right.
\end{aligned}
$$

Substituting Eq. (42) into Eq. (41) yields

$$
\begin{gathered}
\sum(x)=\frac{4 n}{3 v_{T}^{3} \sqrt{2 \pi} x^{3 / 2}} \bar{\Sigma}(x) \\
\bar{\Sigma}(x)=\int_{0}^{x} d x x^{\prime 3 / 2} e^{-x^{\prime}}\left[\Phi^{\prime}(x)-\Phi^{\prime}\left(x^{\prime}\right)\right] \\
+x^{3 / 2} \int_{x}^{\infty} d x^{\prime} e^{-x^{\prime}}\left[\Phi^{\prime}(x)-\Phi^{\prime}\left(x^{\prime}\right)\right]
\end{gathered}
$$

Thus, the electron-electron collision integral reduces to

$$
\delta J_{\mathrm{ee}}[\Phi(x)]=\frac{v_{\mathrm{ei}}}{\bar{Z} f_{\mathrm{M}}} \partial_{v_{k}}\left[v_{k} f_{\mathrm{M}}(v) \frac{\bar{\Sigma}(x)}{x^{3 / 2}}\right]
$$

The next step is to substitute Eq. (45) into Eq. (35) and solve the latter for $\Phi$. To simplify the integration, the right-hand side of Eq. (35) can be rewritten in the form

$$
\phi(x)=\frac{1}{f_{\mathrm{M}}} \partial_{v_{k}}\left[v_{k} f_{\mathrm{M}} \frac{\bar{\phi}(x)}{x^{3 / 2}}\right]
$$

Then, integrating Eq. (35) once, the following integro-differential equation is obtained:

$$
\bar{\phi}=\frac{v_{\mathrm{ei}}}{\overline{\mathrm{Z}}} \bar{\Sigma}(x)
$$

where function $\bar{\phi}$ is related to $\phi$ by integrating Eq. (46),

$$
\bar{\phi}=\frac{e^{x}}{2} \int_{\infty}^{x} d x^{\prime} \phi\left(x^{\prime}\right) \sqrt{x^{\prime}} e^{-x^{\prime}}
$$

To solve Eq. (47) we take the $x$ derivative of both sides of Eq. (47). This gives

$$
\frac{2 \bar{Z}}{3 v_{\mathrm{ei}}} \bar{\phi}^{\prime}=\Phi^{\prime \prime} \gamma\left(\frac{3}{2}, x\right)-\sqrt{x} \int_{x}^{\infty} d x^{\prime} e^{-x^{\prime}} \Phi^{\prime \prime}\left(x^{\prime}\right)
$$

where $\gamma(\alpha, x)=\int_{0}^{x} x^{\prime \alpha-1} e^{-x^{\prime}} d x^{\prime}$ is the incomplete gamma function. Introducing a new function $g(x)=\int_{x}^{\infty} d x^{\prime} \Phi^{\prime \prime}(x) e^{-x^{\prime}}$, Eq. (49) becomes

$$
g(x)=-\frac{2 \bar{Z}}{3 v_{\mathrm{ei}} \gamma(3 / 2, x)} \int_{0}^{x} d x^{\prime} \bar{\phi}^{\prime}\left(x^{\prime}\right) e^{-x^{\prime}}+\frac{C}{\gamma(3 / 2, x)}
$$

where $C$ is the integration constant. Thus, the function $\Phi(x)$ can be expressed as a multiple integral of $\bar{\phi}$ :

$$
\Phi(x)=\bar{C}_{1}+\bar{C}_{2} x-\int d x^{\prime} \int d x^{\prime \prime} e^{x^{\prime \prime}} g^{\prime}\left(x^{\prime \prime}\right)
$$

Next, we report the equations corresponding to the function of the second and third types [Eqs. (36) and (37), respectively]. Equation (36) reduces to

$$
\begin{aligned}
& \frac{x^{3 / 2}}{3} \phi(x)+\frac{\sqrt{\pi} v_{\mathrm{ei}}}{4} \Phi(x)=\frac{v_{\mathrm{ei}}}{\bar{Z}}\left\{\Phi(x)\left[x^{3 / 2} e^{-x}-\gamma\left(\frac{3}{2}, x\right)\right]\right. \\
& +\Phi^{\prime}(x)\left[(1-x) \gamma\left(\frac{3}{2}, x\right)+x^{3 / 2} e^{-x}\right]+\Phi^{\prime \prime}(x) x \gamma\left(\frac{3}{2}, x\right) \\
& +\int_{0}^{x} d x^{\prime} e^{-x^{\prime}} x^{\prime 3 / 2}\left(\frac{2 x^{\prime}}{5}-\frac{1}{3}\right) \Phi\left(x^{\prime}\right) \\
& \left.+x^{3 / 2}\left(\frac{2 x}{5}-\frac{1}{3}\right) \int_{x}^{\infty} d x^{\prime} \Phi\left(x^{\prime}\right) e^{-x^{\prime}}\right\} .
\end{aligned}
$$


Equation (37) for the function of the third type becomes

$$
\begin{aligned}
& \frac{2 x^{3 / 2}}{3} \phi(x)+\frac{3 \sqrt{\pi}}{2} v_{\mathrm{ei}} \Phi(x) \\
& =\frac{v_{\mathrm{ei}}}{\bar{Z}}\left\{\Phi(x)\left[\gamma\left(\frac{5}{2}, x\right)\left(\frac{1}{x}-\frac{10}{3}\right)-\frac{4}{3} x^{3 / 2} e^{-x}\right]\right. \\
& +2 \Phi^{\prime}(x)\left[\gamma\left(\frac{3}{2}, x\right)(2-x)+x^{3 / 2} e^{-x}\right]+2 x \Phi^{\prime \prime}(x) \gamma\left(\frac{3}{2}, x\right) \\
& +\frac{2}{x} \int_{0}^{x} d x^{\prime} x^{\prime 5 / 2} e^{-x^{\prime}} \Phi\left(x^{\prime}\right)\left(\frac{12}{35} x^{\prime}-\frac{2}{15} x-\frac{1}{5}\right) \\
& \left.+2 x^{3 / 2} \int_{x}^{\infty} d x^{\prime} e^{-x^{\prime}}\left(\frac{12}{35} x-\frac{2}{15} x^{\prime}-\frac{1}{5}\right) \Phi\left(x^{\prime}\right)\right\} .
\end{aligned}
$$

The heat transport coefficients in the first approximation depend on functions $\Phi_{13}$ and $\Phi_{14}$, which belong to the function of the second type and can be found by solving Eq. (52) with

$$
\begin{gathered}
\phi(x)=\left(x-\frac{5}{2}\right), \text { for } \Phi=\Phi_{13} / v_{\mathrm{ei}}, \\
\phi(x)=1, \text { for } \Phi=\Phi_{14} / v_{\mathrm{ei}} .
\end{gathered}
$$

To solve the integro-differental equation (52), function $\Phi(x)$ is traditionally expanded ${ }^{10,11}$ in Laguerre polynomials ${ }^{12}$ $\Phi(x)=\sum_{n} A(n) L_{n}^{3 / 2}$. As proposed in Ref. 9, it is more convenient to use a more-generalized expansion in terms of Laguerre polynomials $L_{n}^{\alpha}(x)$. The choice of these polynomials comes from their orthogonal properties

$$
\begin{aligned}
& \int_{0}^{\infty} e^{-x} x^{\alpha} L_{m}^{\alpha}(x) L_{n}^{\alpha}(x) d x \\
& = \begin{cases}0 & \text { if } m \neq n \\
\Gamma(n+\alpha+1) / n ! & \text { if } m=n, \alpha>-1, \quad n=0,1,2, \ldots .\end{cases}
\end{aligned}
$$

Evaluation of the integrals in Eqs. (32) and (33) becomes particularly simple if

$$
\Phi_{13(14)}=x^{\beta} \sum_{n} A(n) L_{n}^{\beta+3 / 2} .
$$

Index $\beta$ is determined by matching the polynomial expansion (57) with the exact solution of $\Phi$ in the limit of $\bar{Z} \rightarrow \infty$. Calculations show that such matching speeds up the convergence of the transport coefficients with the number of polynomials in expansion (57). Taking the limit $\bar{Z} \rightarrow \infty$ in Eq. (52) yields

$$
\left.\Phi\right|_{\bar{Z} \rightarrow \infty}=-\frac{4}{3 \sqrt{\pi} v_{\mathrm{ei}}} x^{3 / 2} \phi
$$

Then, the choice $\beta=3 / 2-k$ with $k=0,1,2, \ldots$ will satisfy the requirement of matching Eq. (57) with the exact solution (58). The parameter $k$ is determined by minimizing the number of terms in the polynomial expansion (1) to match the exact solution for $\bar{Z} \rightarrow \infty$ and (2) to reach the desired accuracy of the transport coefficients for $\bar{Z} \sim 1$. Calculations show that for the case of functions $\Phi_{13}$ and $\Phi_{14}, \beta=1 / 2$ satisfies such a minimization criteria [it takes five terms in Eq. (57) to obtain the transport coefficients with $1 \%$ accuracy]. Therefore, the expansion becomes

$$
\Phi_{13(14)}=\sqrt{x} \sum_{n} A_{13(14)}(n) L_{n}^{2} .
$$

Multiplying Eq. (52) by $x^{3 / 2} e^{-x} L_{s}^{\alpha}(x)$ with $s=0,1,2, \ldots$, $N-1$ [where $N$ is the number of polynomials in the expansion (57)] and integrating the latter in $x$ from 0 until $\infty$, we obtain the system of $N$ algebraic equations. Figure 98.4 shows a dependence of the coefficients $\alpha_{j 1}^{T}$ and $\alpha_{q 1}^{T}$ on the number of polynomials in the expansion (57) with $\beta=-1 / 2, \beta=1 / 2$, and $\beta=3 / 2$, respectively. Observe that the coefficients converge faster with $\beta=1 / 2$.

Next, we derive the numerical coefficient $\alpha_{\sigma}^{E}$ of the stress tensor $\sigma_{i j}^{(1)}$. This requires that Eq. (53) be solved with $\Phi(x)=$ $\Phi_{11}$ and

$$
\phi=-\frac{3 v_{\mathrm{ei}}}{8 x^{3 / 2}}\left\{\frac{\sqrt{\pi}}{2}\left(1+\frac{3}{2 x}\right)+\frac{3}{\bar{Z}}\left[\gamma\left(\frac{3}{2}, x\right)-\frac{1}{x} \gamma\left(\frac{5}{2}, x\right)\right]\right\} .
$$



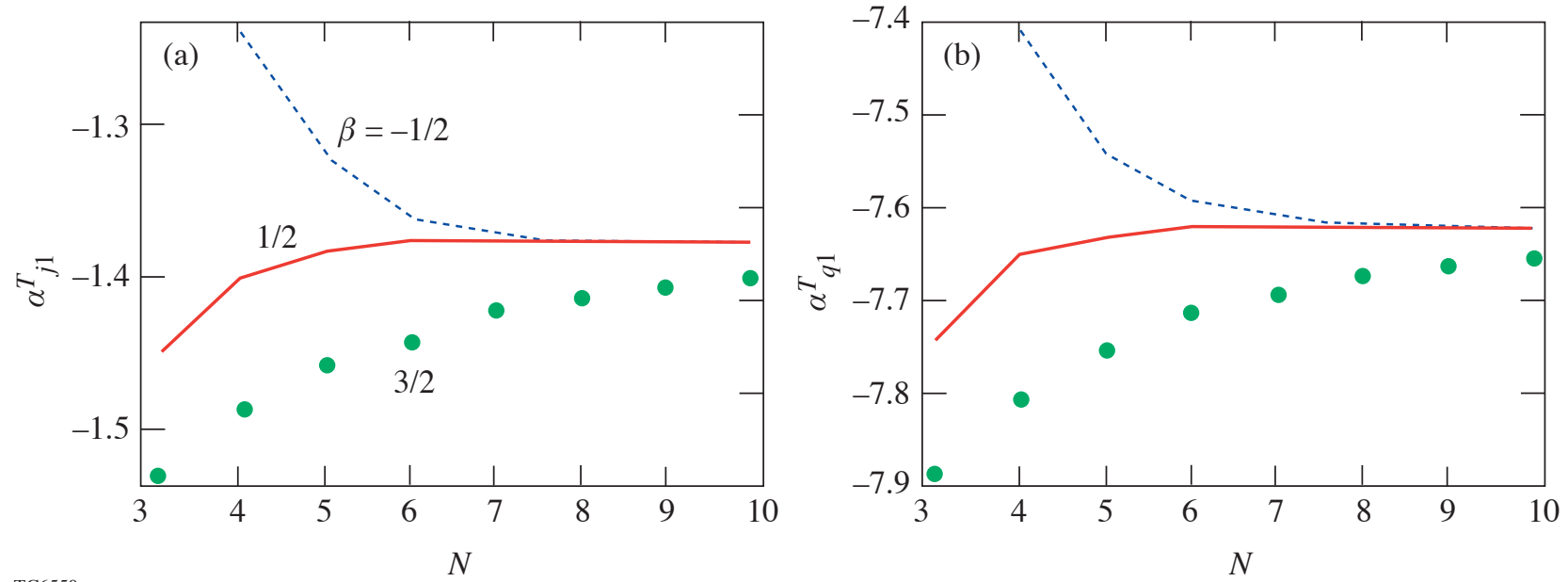

Figure 98.4

Coefficients $\alpha_{j 1}^{T}$ and $\alpha_{q 1}^{T}$ as functions of the number of polynomials in the expansion (57). The results correspond to $\beta=-1 / 2$ (dashed line), $\beta=1 / 2$ (solid line), and $\beta=3 / 2$ (dots).

Similar to the previously considered case, we expand function $\Phi_{11}$ in Laguerre polynomials. To express $\alpha_{\sigma}^{E}$ through just one coefficient in such an expansion, we take

$$
\Phi_{11}=x^{\beta_{1}} \sum_{n} B(n) L_{n}^{\beta_{1}+5 / 2}
$$

The choice of the power index $\beta_{1}$ comes from the condition of matching expansion (61) with the exact solution in the limit of $\bar{Z} \rightarrow \infty$. Neglecting terms proportional to $1 / \bar{Z}$ in Eqs. (53) and (60) gives

$$
\left.\Phi_{11}(x)\right|_{\bar{Z} \rightarrow \infty}=\frac{1}{8 x}+\frac{1}{12} .
$$

It is easy to see that the values $\beta_{1}=-1,-2,-3, \ldots$ satisfy our requirement. Calculations show that expansion (61) with $\beta_{1}=-1$ has the fastest convergence with the number of polynomials. Table 98.II shows a summary of coefficients $\alpha_{\sigma}^{E}$ for a different ion charge $\bar{Z}$. Observe that the stress tensor has a very weak dependence on $\bar{Z}$ (3\% variation in $\alpha_{\sigma}^{E}$ from $\bar{Z}=1$ to $\bar{Z}=\infty$ ). One more function remains to be determined in the first approximation: the correction $\Phi_{12}$ to the symmetric part of the distribution function. This function belongs to the first type and can be found in the integral form using Eq. (51) with

$$
\phi(x)=\left(\frac{3}{2}-x+\frac{3 \sqrt{\pi}}{4 \sqrt{x}}\right) \frac{v_{\mathrm{ei}}}{3}
$$

Table 98.II: Transport coefficients in the first approximation.

\begin{tabular}{|c|c|c|c|c|c|c|c|c|c|}
\hline \hline $\bar{Z}$ & 1 & 2 & 3 & 4 & 5 & 10 & 30 & 80 & $\infty$ \\
\hline$\alpha_{j 1}^{T}$ & -1.39 & -2.1 & -2.57 & -2.91 & -3.16 & -3.87 & -4.59 & -4.89 & -5.09 \\
\hline$\alpha_{q 1}^{T}$ & -7.66 & -12.11 & -15.19 & -17.46 & -19.23 & -24.31 & -29.86 & -32.27 & -33.95 \\
\hline$\alpha_{j 2}^{T}$ & -1.99 & -2.34 & -2.54 & -2.67 & -2.77 & -3.01 & -3.25 & -3.34 & -3.39 \\
\hline$\alpha_{q 2}^{T}$ & -6.35 & -7.93 & -8.90 & -9.57 & -10.07 & -11.40 & -12.70 & -13.23 & -13.58 \\
\hline$\alpha_{\sigma}^{E}$ & 1.029 & 1.027 & 1.023 & 1.020 & 1.017 & 1.010 & 1.004 & 1.002 & 1.000 \\
\hline \hline
\end{tabular}


and $\Phi=\Phi_{12}$. The integration gives ${ }^{8}$

$$
\Phi_{12}(x)=\bar{Z}\left\{-\tilde{C}_{2}+C_{2} x-\frac{\sqrt{\pi}}{12} \int_{1}^{x} \frac{d t \sqrt{t}(x-t)}{[\gamma(3 / 2, t)]^{2}}\right\}
$$

where $C_{2}$ and $\tilde{C}_{2}$ are determined from the condition of zero contribution of $\Phi_{12}$ to the electron density and temperature,

$$
\int_{0}^{\infty} d x e^{-x} \sqrt{x} \Phi_{12}(x)=0, \quad \int_{0}^{\infty} d x e^{-x} x^{3 / 2} \Phi_{12}(x)=0 .
$$

Conditions (65) yield $C_{2}=0.721$ and $\tilde{C}_{2}=0.454$. Note two misprints in $\Phi_{12}$ reported in Ref. 8 [the different sign in front of the integral and $(x-t)$ instead of $(1-t)$ inside the integral]. The correction to the symmetric part in the distribution function comes mainly from balancing the inverse bremsstrahlung heating $\sqrt{\pi / x} v_{\mathrm{ei}} / 3\left(v_{E}^{2} / v_{T}^{2}\right)$ with the electron-electron collisions $\delta J_{\mathrm{ee}}$. Since $\delta J_{\mathrm{ee}} \sim v_{\mathrm{ei}} / \bar{Z}$, function $\Phi_{12}$ becomes proportional to the average ion charge $\bar{Z}$, as shown in Eq. (64). As emphasized in Ref. 8, the symmetric correction $\Phi_{12}$ gives the dominant contribution to the heat flux in the secondorder approximation.

\section{Second-Order Approximation}

Correction $\psi_{2}$ to the distribution function in the second approximation satisfies the following equation: ${ }^{8}$

$$
\begin{aligned}
& \left(x-\frac{5}{2}\right) \frac{\nabla \mathbf{j}^{(1)}}{e n}-\left(x-\frac{3}{2}\right) \frac{2 \nabla \mathbf{q}^{(1)}}{3 n T}+\frac{v^{2}}{3 v_{\mathrm{ei}}}\left[\Phi_{13} \nabla^{2} \ln T\right. \\
& \left.+\Phi_{14} \nabla\left(\nabla \ln n T-\frac{e \mathbf{E}_{0}}{T}\right)\right] \\
& +\mathbf{v} \frac{\nabla v_{E}^{2}}{v_{T}^{2}}\left[\Phi_{12}+\frac{1}{3}\left(\Phi_{13}+\Phi_{14}\right)+\frac{1}{2}-\frac{x}{6}\right] \\
& +\frac{v_{i} \partial_{r_{k}}\left(\mathbf{v}_{E}^{2}\right)_{i k}}{10 v_{T}^{2}}\left(8 x \Phi_{11}-x\right)-\frac{e}{v_{\mathrm{ei}} T} \Phi_{14} \mathbf{v} \partial_{t} \mathbf{E}_{0} \\
& +\frac{\left(\mathbf{v}^{2}\right)_{i j}}{v_{\mathrm{ei}}}\left[\Phi_{13} \frac{\partial^{2} \ln T}{\partial r_{i} \partial r_{j}}+\Phi_{14} \partial_{r_{i}}\left(\partial_{r_{j}} \ln n T-\frac{e E_{0 j}}{T}\right)\right] \\
& +\left(\mathbf{v}^{3}\right)_{i j k} \frac{\partial_{r_{k}}\left(\mathbf{v}_{E}^{2}\right)_{i j}}{v_{T}^{4}}\left(\Phi_{11}-\frac{1}{8}\right)=\delta J_{\mathrm{ei}}\left[\psi_{2}\right]+\delta J_{\mathrm{ee}}\left[\psi_{2}\right] .
\end{aligned}
$$

A general solution of Eq. (66) can be written as

$$
\begin{aligned}
& \psi_{2}=\Phi_{20} \frac{\left(\mathbf{v}^{3}\right)_{i j k} \partial_{r_{k}}\left(\mathbf{v}_{E}^{2}\right)_{i j}}{v_{T}^{4} v_{\mathrm{ei}}}+\Phi_{21} \frac{v_{i} \partial_{r_{j}}\left(\mathbf{v}_{E}^{2}\right)_{i j}}{v_{\mathrm{ei}} v_{T}^{2}} \\
& +\Phi_{22} \frac{\mathbf{v} \nabla v_{E}^{2}}{v_{T}^{2} v_{\mathrm{ei}}}+\frac{\left(\mathbf{v}^{2}\right)_{k j}}{v_{\mathrm{ei}}^{2}}\left\{\Phi_{23}\left(\frac{\partial^{2} \ln T}{\partial r_{k} \partial r_{j}}-\frac{1}{3} \delta_{j k} \nabla^{2} \ln T\right)\right. \\
& \left.+\Phi_{24}\left[\frac{\partial}{\partial_{r_{k}}}\left(\partial_{r_{j}} \ln n T-\frac{e E_{0 j}}{T}\right)-\frac{1}{3} \delta_{j k} \nabla\left(\nabla \ln n T-\frac{e \mathbf{E}_{0}}{T}\right)\right]\right\} \\
& -\Phi_{25} \frac{e}{v_{\mathrm{ei}} T} \mathbf{v} \partial_{t} \mathbf{E}_{0} \\
& +\frac{v_{T}^{2}}{v_{\mathrm{ei}}^{2}}\left[\Phi_{26} \nabla^{2} \ln T+\Phi_{27} \nabla\left(\nabla \ln n T-\frac{e \mathbf{E}_{0}}{T}\right)\right] .
\end{aligned}
$$

The electric current and the heat flux in the second order take the form

$$
\begin{aligned}
j_{i}^{(2)}= & e n v_{T} \lambda_{e}\left[\alpha_{j 1}^{E} \frac{\partial_{r_{k}}\left(\mathbf{v}_{E}^{2}\right)_{i k}}{v_{T}^{2}}+\alpha_{j 2}^{E} \frac{\partial_{r_{i}} v_{E}^{2}}{v_{T}^{2}}\right. \\
& \left.-\alpha_{j 3}^{E} \frac{e \lambda_{e}}{v_{T} T} \partial_{t} E_{0 i}\right], \\
q_{i}^{(2)}= & n T v_{T} \lambda_{e}\left[\alpha_{q 1}^{E} \frac{\partial_{r_{k}}\left(\mathbf{v}_{E}^{2}\right) i k}{v_{T}^{2}}+\alpha_{q 2}^{E} \frac{\partial_{r_{i}} v_{E}^{2}}{v_{T}^{2}}\right. \\
& \left.-\alpha_{q 3}^{E} \frac{e \lambda_{e}}{v_{T}^{T}} \partial_{t} E_{0 i}\right] .
\end{aligned}
$$


Coefficients $\alpha_{j(q)}^{E}$ are calculated using the following relations:

$$
\begin{aligned}
& \alpha_{j 1(2,3)}^{E}=\frac{4}{3 \sqrt{\pi}} \int_{0}^{\infty} d x x^{3 / 2} e^{-x} \Phi_{21(2,5)}(x), \\
& \alpha_{q 1(2,3)}^{E}=\frac{4}{3 \sqrt{\pi}} \int_{0}^{\infty} d x x^{5 / 2} e^{-x} \Phi_{21(2,5)}(x) .
\end{aligned}
$$

Next, we find functions $\Phi_{21}, \Phi_{22}$, and $\Phi_{25}$. These functions are of the second type; therefore, to obtain them we solve Eq. (52) with

$$
\begin{gathered}
\phi=\frac{x}{10}\left(8 \Phi_{11}-1\right), \text { for } \Phi=\Phi_{21} / v_{\mathrm{ei}}, \\
\phi=\Phi_{12}+\frac{1}{3}\left(\Phi_{13}+\Phi_{14}\right)+\frac{1}{2}-\frac{x}{6}, \text { for } \Phi=\Phi_{22} / v_{\mathrm{ei}}, \\
\phi=\Phi_{14}, \text { for } \Phi=\Phi_{25} / v_{\mathrm{ei}} .
\end{gathered}
$$

Following the method described in the previous section, functions $\Phi_{21}, \Phi_{22}$, and $\Phi_{25}$ are expanded in series (57). The exact solution for $\Phi_{21}$ as $\bar{Z} \rightarrow \infty$ becomes

$$
\left.\Phi_{21}\right|_{\bar{Z} \rightarrow \infty}=-\frac{2 x^{3 / 2}}{15 \sqrt{\pi}}\left(1-\frac{x}{3}\right)
$$

thus $\beta$ takes the values $\beta=3 / 2-k$ with $k=0,1,2, \ldots$. The fastest convergence of the coefficients $\alpha_{j 1}^{E}$ and $\alpha_{q 1}^{E}$ is obtained with $\beta=1 / 2$. A summary of $\alpha_{j 1}^{E}$ and $\alpha_{q 1}^{E}$ for different ion charge $\bar{Z}$ is given in Table 98.III. Next, we find the function $\Phi_{22}$. The exact matching of the polynomial expansion (57) with the exact solution $\Phi_{22}$ for $\bar{Z} \rightarrow \infty$,

$$
\left.\Phi_{22}\right|_{\bar{Z} \rightarrow \infty}=-\frac{4 x^{3 / 2}}{3 \sqrt{\pi}} \Phi_{12},
$$

cannot be done since $\Phi_{12}$ does not have a polynomial structure [see Eq. (64)]. It is easy to show, however, that $\Phi_{12}(x \rightarrow 0) \sim 1 / \sqrt{x}$ and $\Phi_{12}(x \rightarrow \infty) \sim x^{5 / 2}$. Therefore, the expansion of $\Phi_{22}$ with $\beta=1$ reproduces the asymptotic limits for $x<<1$ and $x>>1$. Taking $\beta=1$ and keeping $N=5$ terms in expansion (57) gives values of $\alpha_{j 2}^{E}$ and $\alpha_{q 2}^{E}$, which are reported in Table 98.III. Observe that these coefficients become quite large for $\bar{Z}>>1$. To find the remaining coefficients in the heat flux and electric current, we solve the equation for the function $\Phi_{25}$. In the limit of $\bar{Z} \rightarrow \infty$, the function $\Phi_{25}$ becomes

$$
\left.\Phi_{25}\right|_{\bar{Z} \rightarrow \infty}=-\left.\Phi_{14}\right|_{\bar{Z} \rightarrow \infty} \frac{4 x^{3 / 2}}{3 \sqrt{\pi}}=\frac{16}{9 \pi} x^{3} ;
$$

thus, $\beta=3,2,1 \ldots$ matches the polynomial expansion (57) with the exact solution in the limit of $\bar{Z} \rightarrow \infty$. Calculations show that $\beta=1$ requires a minimum number of polynomials in expansion (57) to achieve the desired accuracy. The values of $\alpha_{j 3}^{E}$ and $\alpha_{q 3}^{E}$ are summarized in Table 98.III. Next, we

Table 98.III: Transport coefficients in the second approximation.

\begin{tabular}{|c|c|c|c|c|c|c|c|c|c|}
\hline \hline $\bar{Z}$ & 1 & 2 & 3 & 4 & 5 & 10 & 30 & 80 & $\infty$ \\
\hline$\alpha_{j 1}^{E}$ & -0.03 & -0.01 & 0.00 & 0.02 & 0.03 & 0.06 & 0.09 & 0.10 & 0.11 \\
\hline$\alpha_{q 1}^{E}$ & -0.03 & 0.07 & 0.16 & 0.23 & 0.30 & 0.49 & 0.73 & 0.83 & 0.90 \\
\hline$\alpha_{j 2}^{E}$ & 4.05 & 8.54 & 13.07 & 17.51 & 21.86 & 42.30 & 116.3 & $\bar{Z} 3.66$ & $\bar{Z} 3.48$ \\
\hline$\alpha_{q 2}^{E}$ & 19.7 & 48.3 & 80.0 & 113.0 & 146.6 & 314.1 & 960.9 & $\bar{Z} 31.7$ & $\bar{Z} 31.3$ \\
\hline$\alpha_{j 3}^{E}$ & 4.69 & 7.21 & 9.07 & 10.51 & 11.67 & 15.15 & 19.18 & 21.00 & 22.28 \\
\hline$\alpha_{q 3}^{E}$ & 16.77 & 28.75 & 38.45 & 46.39 & 52.99 & 74.05 & 100.6 & 113.3 & 122.5 \\
\hline \hline
\end{tabular}


combine the electric current and the thermal flux in the first and second approximations. The result is

$$
\begin{aligned}
& \mathbf{j}_{i}= e n v_{T} \lambda_{e}\left[\alpha_{j 1}^{T} \partial_{r_{i}} \ln T+\alpha_{j 2}^{T}\left(\partial_{r_{i}} \ln n T-\frac{e E_{0 i}}{T}\right)\right. \\
&\left.+\alpha_{j 1}^{E} \frac{\partial_{r_{k}}\left(\mathbf{v}_{E}^{2}\right)_{i k}}{v_{T}^{2}}+\alpha_{j 2}^{E} \frac{\partial_{r_{i}} v_{E}^{2}}{v_{T}^{2}}-\alpha_{j 3}^{E} \frac{e \lambda_{e}}{v_{T} T} \partial_{t} E_{0 i}\right], \\
& \mathbf{q}_{i}=n T v_{T} \lambda_{e}\left[\alpha_{q 1}^{T} \partial_{r_{i}} \ln T+\alpha_{q 2}^{T}\left(\partial_{r_{i}} \ln n T-\frac{e E_{0 i}}{T}\right)\right. \\
&\left.+\alpha_{q 1}^{E} \frac{\partial_{r_{k}}\left(\mathbf{v}_{E}^{2}\right)_{i k}}{v_{T}^{2}}+\alpha_{q 2}^{E} \frac{\partial_{r_{i}} v_{E}^{2}}{v_{T}^{2}}-\alpha_{q 3}^{E} \frac{e \lambda_{e}}{v_{T} T} \partial_{t} E_{0 i}\right] .
\end{aligned}
$$

Imposing a condition of zero current $\mathbf{j}=0$ and also assuming $t_{E} v_{\mathrm{ei}} \ll<\alpha_{j 3}^{E} / \alpha_{j 2}^{T}$ (where $t_{E}$ is the time scale of $\mathbf{E}_{0}$ variation) define the slowly varying component of the electric field $\mathbf{E}_{0}$,

$$
\begin{aligned}
\frac{e E_{0 i}}{T}= & \partial_{r_{i}} \ln n T+\frac{\alpha_{j 1}^{T}}{\alpha_{j 2}^{T}} \partial_{r_{i}} \ln T+\frac{\alpha_{j 1}^{E}}{\alpha_{j 2}^{T}} \frac{\partial_{r_{k}}\left(\mathbf{v}_{E}^{2}\right)_{i k}}{v_{T}^{2}} \\
& +\frac{\alpha_{j 2}^{E}}{\alpha_{j 2}^{T}} \frac{\partial_{r_{i}} v_{E}^{2}}{v_{T}^{2}}
\end{aligned}
$$

Substituting $\mathbf{E}_{0}$ from Eq. (80) into Eq. (79) gives the heat flux in laser-produced plasmas,

$\mathbf{q}_{i}=n T v_{T} \lambda_{e}\left[\beta^{T} \partial_{r_{i}} \ln T+\beta_{1}^{E} \frac{\partial_{r_{i}} v_{E}^{2}}{v_{T}^{2}}+\beta_{2}^{E} \frac{\partial_{r_{k}}\left(\mathbf{v}_{E}^{2}\right)_{i k}}{v_{T}^{2}}\right]$

where

$$
\beta^{T}=\alpha_{q 1}^{T}-\alpha_{q 2}^{T} \alpha_{j 1}^{T} / \alpha_{j 2}^{T}
$$

$$
\beta_{1}^{E}=\alpha_{q 2}^{E}-\alpha_{q 2}^{T} \alpha_{j 2}^{E} / \alpha_{j 2}^{T},
$$

and

$$
\beta_{2}^{E}=\alpha_{q 1}^{E}-\alpha_{q 2}^{T} \alpha_{j 1}^{E} / \alpha_{j 2}^{T}
$$

can be represented with the following fitting formulas:

$$
\begin{gathered}
\beta^{T}=-\frac{128}{3 \pi} \frac{\bar{Z}+0.24}{\bar{Z}+4.20}, \\
\beta_{1}^{E}=17.31 \bar{Z} \frac{\bar{Z}^{2}+14.04 \bar{Z}+2.41}{\bar{Z}^{2}+14.34 \bar{Z}+29.5}, \\
\beta_{2}^{E}=0.45 \frac{\bar{Z}-0.29}{\bar{Z}+3.47} .
\end{gathered}
$$

In addition, the coefficients in the electric field $E_{0}$ can be fitted as follows:

$$
\begin{aligned}
& \frac{\alpha_{j 1}^{T}}{\alpha_{j 2}^{T}}=1.50 \frac{\bar{Z}+0.52}{\bar{Z}+2.26}, \\
& \frac{\alpha_{j 1}^{E}}{\alpha_{j 2}^{T}}=-0.03 \frac{\bar{Z}-2.63}{\bar{Z}+3.22}, \\
& \frac{\alpha_{j 2}^{E}}{\alpha_{j 2}^{T}}=-1.03 \bar{Z} \frac{\bar{Z}+8.54}{\bar{Z}+3.82} .
\end{aligned}
$$

Coefficients $\beta^{T}$ and $\alpha_{j 1}^{T} / \alpha_{j 2}^{T}$ agree with previously published results. ${ }^{1,11,13}$

Next, we discuss the validity of the derived transport coefficients. As shown earlier, the main contribution to the second-order heat flux comes from the correction $\Phi_{12}$ to the symmetric part of the distribution function. The function $\Phi_{12}$ is given in the integral form by Eq. (64) and has the following asymptotic behavior for small and large velocities: 


$$
\begin{gathered}
\Phi_{12}(x \rightarrow 0)=-\frac{\bar{Z}}{4} \sqrt{\frac{\pi}{x}}, \\
\Phi_{12}(x \rightarrow \infty)=-\bar{Z} \frac{4}{45 \sqrt{\pi}} x^{5 / 2} .
\end{gathered}
$$

The validity condition of the Chapman-Enskog method ${ }^{10}$ $\left|\Phi_{12}\right| v_{E}^{2} / v_{T}^{2} \ll 1$ breaks down for

$$
x<\bar{Z}^{2} \frac{\pi}{16} \frac{v_{E}^{4}}{v_{T}^{4}}
$$

and

$$
x>\frac{3}{\bar{Z}^{2 / 5}}\left(\frac{v_{T}}{v_{E}}\right)^{4 / 5}
$$

According to Eq. (71), the main contribution to the heat flux comes from the superthermal electrons [which correspond to the maximum in the function $\left.x^{5 / 2} e^{-x} \Phi(x)\right]$. Therefore, the limit (87) imposes no restrictions on the applicability of the derived results. The electron distribution function for the subthermal electrons, nevertheless, is different from the limit (85). As derived in Refs. 14 and 15, the inverse bremsstrahlung heating modifies the distribution of the cold electrons to

$$
f_{0}\left(v \ll v_{T}\right)=\frac{n}{(2 \pi)^{3 / 2} v_{T}^{3}} \exp \left(-\frac{1}{v_{T}^{2}} \int_{0}^{v} \frac{u^{4} d u}{u^{3}+V_{L}^{3}}\right),
$$

where $V_{L}=\left(\sqrt{\pi / 8} \bar{Z} v_{E}^{2} v_{T}\right)^{1 / 3}$ is the Langdon velocity. ${ }^{16}$ To check the limitations due to the second condition (88), we find that the maximum of

$$
x^{5 / 2} e^{-x} \Phi_{22} \sim x^{5 / 2} e^{-x} x^{3 / 2} \Phi_{12} \sim x^{13 / 2} e^{-x}
$$

corresponds to $x_{\max } \simeq 13 / 2$. This limits the applicability of the Chapman method to $v_{E}^{2} / v_{T}^{2}<0.2 / \bar{Z}$. Even though the modulus of $\Phi_{12}$ becomes larger than unity for large $x$ [see Eq. (88)], we can show that

$$
f_{0}^{\text {int }}=f_{\mathrm{M}} \exp \left[1+\frac{v_{E}^{2}}{v_{T}^{2}} \Phi_{12}(x)\right]
$$

is a good approximation to the symmetric part of the distribution function even for $x \rightarrow \infty$. For such a purpose, we find the asymptotic behavior of the function that satisfies the following equation:

$$
\partial_{t} f_{0}=J_{\mathrm{ee}}\left(f_{0}, f_{0}\right)
$$

We look for a solution of Eq. (91) in the form $f_{0}=A e^{\Psi}$, where $\Psi=F\left(v_{T}^{2}\right) g\left(v^{2}\right)$ and $A$ is a normalization constant. The temperature dependence is combined in function $F$, and velocity dependence is in $g$; then, the time derivative of $f_{0}$ becomes

$$
\partial_{t} f_{0}=f_{0} F^{\prime} v_{T}^{2} g \frac{\partial_{t} T}{T}=f_{0} F^{\prime} g \frac{v_{E}^{2} v_{\mathrm{ei}}}{3},
$$

where we substituted $\partial_{t} T / T=\left(v_{E}^{2} / v_{T}^{2}\right) v_{\mathrm{ei}} / 3$ due to the inverse bremsstrahlung heating. The electron-electron collision integral reduces in this case to

$$
J_{\mathrm{ee}}=\frac{16 \pi e^{4} \Lambda}{3 m^{2} v} F \frac{\partial}{\partial v^{2}}\left[f_{0} I\left(v^{2}\right)\right]
$$

$$
\begin{aligned}
I\left(v^{2}\right)= & 4 \pi \int_{0}^{v} d v^{\prime} v^{\prime 4} f_{0}\left(v^{\prime}\right)\left[g^{\prime}\left(v^{2}\right)-g^{\prime}\left(v^{\prime 2}\right)\right] \\
& +4 \pi v^{3} \int_{v}^{\infty} d v^{\prime} v^{\prime} f_{0}\left(v^{\prime}\right)\left[g^{\prime}\left(v^{2}\right)-g^{\prime}\left(v^{\prime 2}\right)\right] .
\end{aligned}
$$

In the limit of $v \rightarrow \infty, I$ becomes

$$
I \simeq g^{\prime}\left(v^{2}\right) 4 \pi \int_{0}^{\infty} d v^{\prime} v^{\prime 4} f_{0}\left(v^{\prime}\right)=3 g^{\prime}\left(v^{2}\right)_{n v_{T}^{2}},
$$

and Eq. (91) takes the form

$$
g^{\prime \prime}+F g^{\prime 2}=\bar{Z} \frac{v v_{E}^{2}}{v_{T}^{5}} \frac{F^{\prime}}{F} \frac{g}{18 \sqrt{2 \pi}} .
$$


Next, we make an assumption $g^{\prime \prime}<g^{\prime 2}$, which will be verified a posteriori. In this case the solution of Eq. (95) becomes

$$
g=\frac{2}{225 \sqrt{2 \pi}} v^{5} \frac{F^{\prime}}{F^{2}} \frac{\bar{Z} v_{E}^{2}}{v_{T}^{5}}
$$

Observe that the condition $g^{\prime \prime} \ll g^{\prime 2}$ is satisfied in the limit of large velocity. The function $g$, by definition, does not depend on temperature; this yields for $F$

$$
\frac{F^{\prime}}{F^{2}}=C\left(v_{T}^{2}\right)^{5 / 2}, \quad F=\frac{\tilde{C}}{v_{T}^{7}},
$$

where $C$ and $\tilde{C}$ are constants. The distribution function $f_{0}$ depends on the product $F\left(v_{T}^{2}\right) g\left(v^{2}\right)$, which, according to Eqs. (96) and (97), takes the form

$$
F\left(v_{T}^{2}\right) g\left(v^{2}\right)=-\frac{7 \bar{Z}}{225 \sqrt{2 \pi}} \frac{v_{E}^{2} v^{5}}{v_{T}^{7}}
$$

Using Eq. (98), the asymptotic limit of the symmetrical part of the distribution function reduces to

$$
f_{0}(x>>1) \sim \exp \left(-0.07 \bar{Z} \frac{v_{E}^{2}}{v_{T}^{2}} x^{5 / 2}\right) .
$$

The latter equation must be compared to $f_{0}^{\text {int }}$ in the limit $x \rightarrow \infty$ [see Eq. (90)],

$$
f_{0}^{\text {int }}(x>>1) \sim \exp \left(-0.05 \bar{Z} \frac{v_{E}^{2}}{v_{T}^{2}} x^{5 / 2}\right)
$$

Thus, we can conclude that the function in the form (90) is a good approximation to the distribution function for thermal and superthermal electrons.

In conclusion, we have derived the transport coefficients, including the thermal and ponderomotive terms for an arbitrary ion charge. The modification of the thermal transport due to the ponderomotive effects near the critical surface and laser turning point will be discussed in a forthcoming publication.

\section{ACKNOWLEDGMENT}

This work was supported by the U.S. Department of Energy Office (DOE) of Inertial Confinement Fusion under Cooperative Agreement No. DE-FC0392SF19460, the University of Rochester, and the New York State Energy Research and Development Authority. The support of DOE does not constitute an endorsement by DOE of the views expressed in this article.

\section{REFERENCES}

1. L. Spitzer, Jr. and R. Härm, Phys. Rev. 89, 977 (1953).

2. R. C. Malone, R. L. McCrory, and R. L. Morse, Phys. Rev. Lett. 34, 721 (1975).

3. I. B. Bernstein, C. E. Max, and J. J. Thomson, Phys. Fluids 21, 905 (1978).

4. P. Mora and R. Pellat, Phys. Fluids 22, 2408 (1979).

5. I. P. Shkarofsky, Phys. Fluids 23, 52 (1980).

6. V. L. Ginzburg, Propagation of Electromagnetic Waves in Plasmas, edited by W. L. Sadowski and D. M. Gallik (Gordon and Breach, New York, 1961).

7. V. I. Perel' and Ya. M. Pinskii, Sov. Phys.-JETP 27, 1014 (1968).

8. A. V. Maxsimov, V. P. Silin, and M. V. Chegotov, Sov. J. Plasma Phys. 16, $331(1990)$

9. V. N. Goncharov and V. P. Silin, Plasma Phys. Rep. 21, 48 (1995).

10. S. Chapman and T. G. Cowling, The Mathematical Theory of NonUniform Gases; An Account of the Kinetic Theory of Viscosity, Thermal Conduction and Diffusion in Gases, 3rd. ed. (Cambridge University Press, Cambridge, England, 1970).

11. S. I. Braginskii, in Reviews of Plasma Physics, edited by Acad. M. A. Leontovich (Consultants Bureau, New York, 1965), Vol. 1.

12. N. N. Lebedev and R. A. Silverman, Special Functions and Their Applications, rev. English ed. (Dover Publications, New York, 1972).

13. E. M. Epperlein and M. G. Haines, Phys. Fluids 29, 1029 (1986).

14. A. V. Maksimov et al., JETP 86, 710 (1998).

15. V. P. Silin, Phys.-Usp. 45, 955 (2002).

16. A. B. Langdon, Phys. Rev. Lett. 44, 575 (1980). 


\section{Effects of Nonuniform Illumination on Implosion Asymmetry in Direct-Drive Inertial Confinement Fusion}

Attaining ignition and high gain in inertial confinement fusion (ICF) requires that deuterium-tritium (DT)-filled capsules be spherically imploded to high temperature and density. ${ }^{1-3}$ "Hotspot" ignition, where a capsule is compressed so as to form two different regions - a small mass of low-density, hot ( $10-\mathrm{keV})$ fuel at the center surrounded by a larger mass of high-density, low-temperature fuel-is the leading method envisioned to achieve this goal. Shock coalescence "ignites" the hot spot, and a burn wave propagates into the main fuel region. Success requires a symmetric implosion because significant deviation from spherical symmetry will result in shock dynamics that do not lead to ignition. In the direct-drive approach to ICF, where implosion occurs in response to a large number of high-power, individual laser beams illuminating the surface of a capsule, the requirement for spherical implosion imposes severe constraints on the uniformity of the laser drive $e^{1-3}$ and on the sphericity of the capsule.

Illumination nonuniformities, coupled with initial capsule imperfections, lead to distortions in the compressed capsule. High-mode-number perturbations $(\ell>10)$ are primarily imprinted by nonuniformities within individual laser beams. ${ }^{4,5}$ During both the acceleration and deceleration phases, these perturbations are amplified by Rayleigh-Taylor (RT) instabilities and grow exponentially until reaching saturation at amplitudes of $\sim 4 R / \ell^{2}$ ( $R$ is the capsule radius); thereafter, they grow linearly. ${ }^{6}$ Low-mode-number asymmetries $(\ell \leq 10)$ result primarily from either drive-pressure asymmetry, due to nonuniformity in on-target laser intensity, or capsule fabrication asymmetry. ${ }^{4,5}$ These secular modes grow linearly throughout the entire implosion, largely due to Bell-Plesset(BP)-related convergence effects. ${ }^{7}$

A major effort has been made in current ICF research to reduce target illumination nonuniformity and capsule imperfections. Characterization of these efforts requires the measurement of any deviations from spherical symmetry in the assembled capsule mass, or areal density $(\rho R) .{ }^{8}$ Previous work relied on numerical simulations to predict the conditions under which asymmetries may develop and on x-ray imaging to provide information about emission symmetry. ${ }^{9}$ Quantitative experimental information about $\rho R$ asymmetries has not been available, however, until recent experiments ${ }^{10-11}$ on OMEGA ${ }^{12}$ using novel charged-particle spectrometry techniques. ${ }^{13}$ These experiments have resulted in the first studies of low-modenumber $\rho R$ asymmetries at the time of fusion burn for directdrive spherical implosions (the diagnostic technique is sensitive to structure with mode numbers $\ell \leqslant 5$ ). From these experiments we conclude that changes in laser-intensity distributions result in changes in $\rho R$ asymmetries, while capsule imperfections do not seem to be a dominant factor under current conditions. ${ }^{14}$ In this article we present new studies showing quantitatively, for the first time, how the amplitude of $\rho R$ asymmetries is directly correlated with the amplitude of asymmetries in time-averaged, on-target laser intensity $I$ for ablatively driven implosions. The resulting scaling law is based on both theoretical implications of capsule convergence and experimental data and has implications for future work on the National Ignition Facility (NIF) ${ }^{1}$ as well as on OMEGA. The terminology that will be used is that $\langle\rho R\rangle$ and $\langle I\rangle$ are averages over angle, $\delta \rho R$ and $\delta I$ are deviations from the average at a given angle, and $\langle\delta \rho R\rangle$ and $\langle\delta I\rangle$ are rms averages over angle.

Illumination asymmetries on OMEGA are generated by several sources. First, there are differences in the time-integrated energies delivered by the 60 individual laser beams, which can be characterized by an rms beam energy imbalance $\sigma_{b}$ that tends to be in the range $\sigma_{b} \lesssim 3 \% .^{15}$ The beams, however, overlap on the capsule surface, and the overlap reduces the net energy nonuniformity to a value $\sigma_{e}$ that can be estimated from a typical measured beam profile shape and the theoretical positions of the individual beam centers: $\sigma_{e} \sim 0.8 \%$ to $1.5 \%$ rms. The total illumination nonuniformity on the capsule surface is higher than $\sigma_{e}$ because of other contributing factors and can be estimated as $\langle\delta I\rangle /\langle I\rangle \approx \sqrt{\sigma_{e}^{2}+\sigma_{s}^{2}+\sigma_{p}^{2}+\sigma_{0}^{2}}$. The component $\sigma_{s}$, typically $\sim 1 \% \mathrm{rms},{ }^{16}$ is due to deviations of individual beam shape profiles from that assumed in calculating $\sigma_{e}$. The component $\sigma_{p}$ (typically $\sim 1.9 \% \mathrm{rms}^{16}$ ) results from errors in the pointing of individual laser beams, and $\sigma_{0}$ (typically $\sim 1 \% \mathrm{rms}^{16}$ ) is an additional contribution from any 
offset of the capsule center from the center of the target chamber ( $\sigma_{0}$ is $\sim 0.2$ times the offset in $\mu \mathrm{m}^{16}$ ). Target offset results in drive asymmetry with strong $\ell=1$ and 2 modes. This is demonstrated in Fig. 98.5, which displays a two-dimensional (2-D) simulation from the hydrodynamic code $D R A C O$, incorporating the measured beam imbalance and other nominal experimental conditions for an implosion with $50-\mu \mathrm{m}$ offset from target chamber center (TCC). The simulation indicates a strong correlation between drive asymmetry and $\rho R$ asymmetry, which will be addressed empirically below. These low-mode capsule perturbations cannot be smoothed by the effects of lateral energy flow in the form of transverse thermal conduction ${ }^{17}$ because the scale length of the perturbations is typically much longer than the separation between the critical surface and the ablation surface.
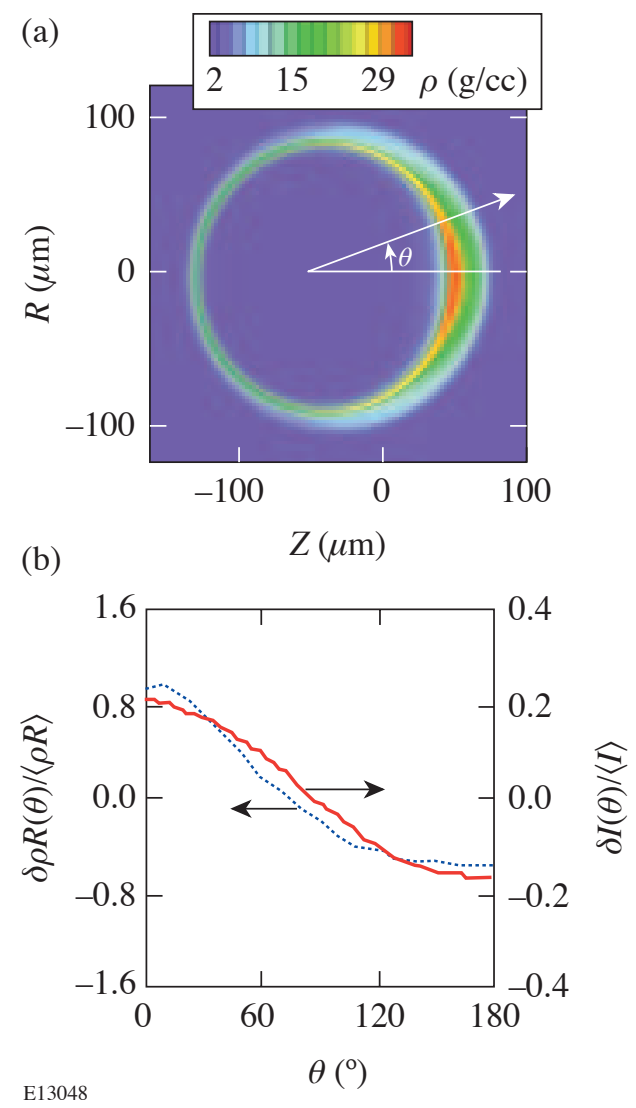

\section{Figure 98.5}

(a) Density contours at a time of peak burn ( $\sim 1.9 \mathrm{~ns})$ for an implosion of a capsule offset by $50 \mu \mathrm{m}$ from TCC, simulated using the 2-D code DRACO for conditions of shot $26646(23 \mathrm{~kJ}$ of laser energy in a 1-ns square pulse applied to a capsule with $15 \mathrm{~atm}$ of $\mathrm{D}_{2}$ in a $20-\mu \mathrm{m} \mathrm{CH}$ shell). (b) The target offset results in drive asymmetry with strong $\ell=1$ and 2 modes, which generates a correlated $\rho R$ asymmetry.
The amplitude of asymmetries in $\rho R$ is quantitatively correlated with the amplitude of asymmetries in time-averaged, on-target laser intensity $I$. Both theoretical and experimental approaches have been used to understand the correlation. Theoretically, a scaling law for predicting how measured $\rho R$ asymmetries relate to $\langle\delta I\rangle /\langle I\rangle$ can be derived from considerations of implosion dynamics, assuming that $\rho R$ asymmetries are seeded by the illumination asymmetries and modified due to effects of capsule convergence. The growth rates of low- $\ell$ number perturbations due to RT instabilities are small $(\propto \sqrt{\ell})$. To first order, the angular variations in acceleration rates $(g)$ during both acceleration and deceleration phases of an implosion can be written $\langle\delta g\rangle /\langle g\rangle \approx\left\langle\delta V_{\text {imp }}\right\rangle /\left\langle V_{\text {imp }}\right\rangle$ (Ref. 1), where $V_{\text {imp }}=V_{\text {imp }}(t)$ is the capsule implosion velocity. Starting with $\left(R_{0}-R\right) \approx V_{\text {imp }} t_{\mathrm{imp}}$ and considering implosion dynamics, one obtains

$$
-\frac{\langle\delta R\rangle}{\langle R\rangle} \approx \frac{\left\langle\delta V_{\mathrm{imp}}\right\rangle}{\left\langle V_{\mathrm{imp}}\right\rangle}\left(C_{r}-1\right) .
$$

In this expression, $C_{r}$ is the convergence ratio

$$
C_{r} \equiv R_{0} /\langle R\rangle \approx \sqrt{\langle\rho R\rangle / f \rho_{0} R_{0}},
$$

where $\rho_{0}$ and $R_{0}$ are the initial shell density and radius and $f$ is the fraction of shell mass not ablated (which can be estimated from "burnthrough" experiments ${ }^{18}$ ). One obtains $-\langle\delta R\rangle /\langle R\rangle \approx 1 / 2\langle\delta \rho R\rangle /\langle\rho R\rangle . V_{\text {imp }}$ is a function of laser intensity on target for direct-drive implosions with

$$
V_{\mathrm{imp}} \propto I^{1 / 3} \ln \left(m_{0} / m\right)
$$

where $m\left(m_{0}\right)$ is the payload (initial) capsule mass determined by $d m / d t \propto I^{\alpha}$ ( $\alpha$ is a constant). ${ }^{1,19,20}$ Substituting these relations into Eq. (1) and keeping terms of first order in $\langle\delta I\rangle /\langle I\rangle$, the resultant scaling has the form

$$
\langle\delta \rho R\rangle /\langle\rho R\rangle \approx B\left(C_{r}-1\right)\langle\delta I\rangle /\langle I\rangle,
$$

where $B$ is a coefficient of the order of 1 that depends weakly on the payload mass. This result is analogous to the BP effect for incompressible fluids, which predicts that the growth of $\delta \rho R$ is proportional to capsule convergence and in-flight shell thickening. The above discussion includes only 1-D effects. 
Two-dimensional (2-D) effects, such as lateral mass flow, can modify the convergence-driven asymmetry growth, resulting in a lower value of $B$ for high- $\ell$-number asymmetries with ratios of perturbation wavelength $(\lambda)$ to in-flight shell thickness $(\Delta)$ of the order of 1 or less. To avoid this theoretical complication, we use experimental data to determine $B$. In addition, since initial asymmetries in capsule structure due to fabrication imperfections (with rms amplitude $\sigma_{C}$ ) should grow in the same manner during convergence, we would expect that

$$
\left(C_{r}-1\right)^{-2}\left(\frac{\langle\delta \rho R\rangle}{\langle\rho R\rangle}\right)^{2} \approx a^{2} \sigma_{C}^{2}+B^{2}\left(\frac{\langle\delta I\rangle}{\langle I\rangle}\right)^{2}
$$

where $a$ is an unknown coefficient. Experimental verification of the form of this equation, and a value for $B$, will be found below.

Experiments were conducted on OMEGA with 60 beams of frequency-tripled $(0.35-\mu \mathrm{m}) \mathrm{UV}$ light driving the targets directly. The total laser energy was $\sim 23 \mathrm{~kJ}$ for 1 -ns square pulses or $\sim 18 \mathrm{~kJ}$ for shaped pulses. Individual beams were smoothed using single-color-cycle, 1-THz, 2-D smoothing by spectral dispersion (SSD) and polarization smoothing (PS) using birefringent wedges. ${ }^{5}$ The room-temperature capsules had plastic (CH) shells with 20- $\mu$ m nominal thickness and were filled with $18 \mathrm{~atm}$ of $\mathrm{D}^{3} \mathrm{He}$ or $15 \mathrm{~atm}$ of $\mathrm{D}_{2}$ gas. Each imploded cryogenic capsule had an 80 - to $100-\mu \mathrm{m} \mathrm{D}_{2}$-ice layer inside an $\sim 5-\mu \mathrm{m} \mathrm{CH}$ shell. ${ }^{21,22}$ The primary ${ }^{23}$ or secondary protons ${ }^{10}$ generated from $\mathrm{D}^{3} \mathrm{He}$ reactions $\left(\mathrm{D}+{ }^{3} \mathrm{He} \rightarrow \alpha+p\right)$ were measured. These protons are energetic enough to easily penetrate the $\mathrm{CH}$ shell or $\mathrm{D}_{2}$-ice layer, but they interact strongly enough with the capsule plasma that their energy loss is a direct measure of $\rho R$ for each spectrometer line of sight:

$$
\rho R=\int_{E_{0}}^{E} \rho(d E / d x)^{-1} d E
$$

[see (Ref. 24)]. Because the shell ( $\mathrm{CH}$ or $\mathrm{D}_{2}$ ice) has a lower temperature, higher density, and higher mass than the gas, the measured $\rho R$ and $\rho R$ asymmetry are dominated by $\rho R_{\text {shell }}$.

$\rho R$ asymmetry can be seen in sample $\mathrm{D}^{3} \mathrm{He}$ proton spectra from a single shot (25221), shown in Fig. 98.6. The measured mean proton energy losses $\Delta\left\langle E_{p}\right\rangle$ varied from 1.1 to $2.2 \mathrm{MeV}$, leading to a variation in $\rho R$ from about 35 to $70 \mathrm{mg} / \mathrm{cm}^{2}$. Under current conditions a number of sources of $\rho R$ asymmetry typically contribute, with no single source dominating. When an effort is made to maintain the same capsule and laser conditions from shot to shot, the spatially averaged $\langle\rho R\rangle$ remains relatively constant, as shown in Fig. 98.7. Although contiguous implosions often show similar angular variations in $\delta \rho R,{ }^{10,11}$ there are small, random variations from shot to shot and a tendency for the angular variations to become uncorrelated over a long shot series.

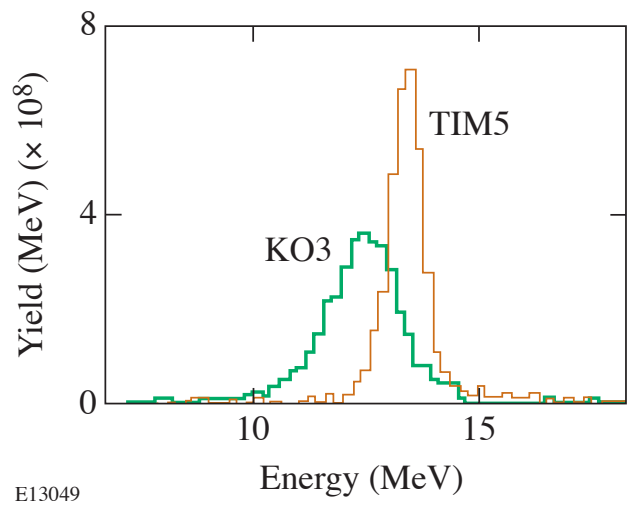

Figure 98.6

Proton spectra were measured simultaneously at seven different diagnostic ports for shot 25221. Two of the spectra are shown here, labeled with the port ID. Substantial asymmetries in the mean downshifted energy indicate $\rho R$ asymmetry.

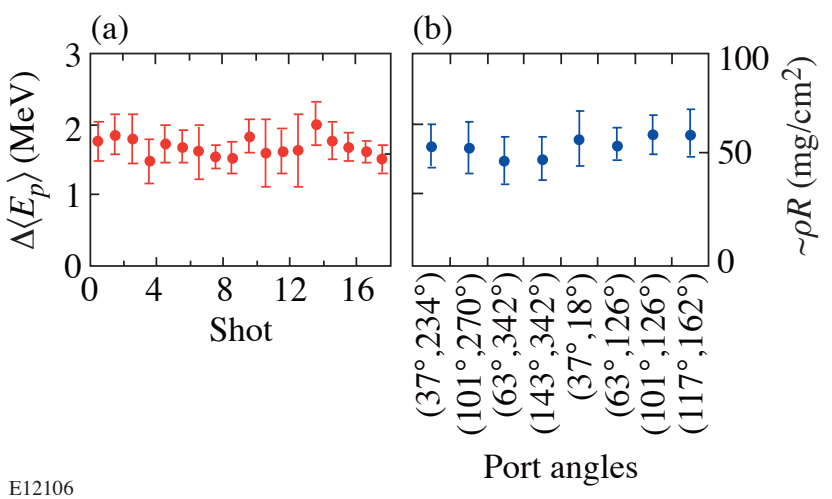

Figure 98.7

(a) Measured $\Delta\left\langle E_{p}\right\rangle$ and inferred $\rho R$ for individual shots that were nominally identical, averaged over different port locations, plotted versus time over a two-week time interval. (b) Measured $\Delta\left\langle E_{p}\right\rangle$ and inferred $\rho R$ at different port angles $(\theta, \varphi)$, averaged over the same shots over a two-week interval. The "error bars" are not measurement uncertainties but standard deviations of all measurements represented by a given, plotted average. 
For the shots under study here, the values of $\langle\delta \rho R\rangle /\langle\rho R\rangle$ and $\langle\delta I\rangle /\langle I\rangle$ were tabulated and are plotted in Fig. 98.8. The data were fit to the two-parameter function

$$
\left(C_{r}-1\right)^{-1}\langle\delta \rho R\rangle /\langle\rho R\rangle=\sqrt{A^{2}+B^{2}(\langle\delta I\rangle /\langle I\rangle)^{2}}
$$

this is equivalent to Eq. (2) with $A^{2}=\left\langle a^{2} \sigma_{C}^{2}\right\rangle$, but $A^{2}$ can also be thought of as including the average effect of any other unknown source of asymmetry not correlated with $\langle\delta I\rangle /\langle I\rangle$. As discussed in the figure caption, the data are well fit by this function using a value of $B \approx 1 / 2$, indicating that the contribution of $\langle\delta I\rangle /\langle I\rangle$ to $\langle\delta \rho R\rangle /\langle\rho R\rangle$ is

$$
\frac{\langle\delta \rho R\rangle}{\langle\rho R\rangle} \approx \frac{1}{2}\left(C_{r}-1\right) \frac{\langle\delta I\rangle}{\langle I\rangle} .
$$

The value $B=1 / 2$ in Eq. (3) was determined almost exclusively by the high $\langle\delta I\rangle /\langle I\rangle$, high $\langle\delta \rho R\rangle /\langle\rho R\rangle$ data points in Fig. 98.8. Most of these points correspond to large capsule offset, where illumination asymmetries are dominated by $\ell=$ 1 and $\ell=2$; the others correspond to cases with some laser

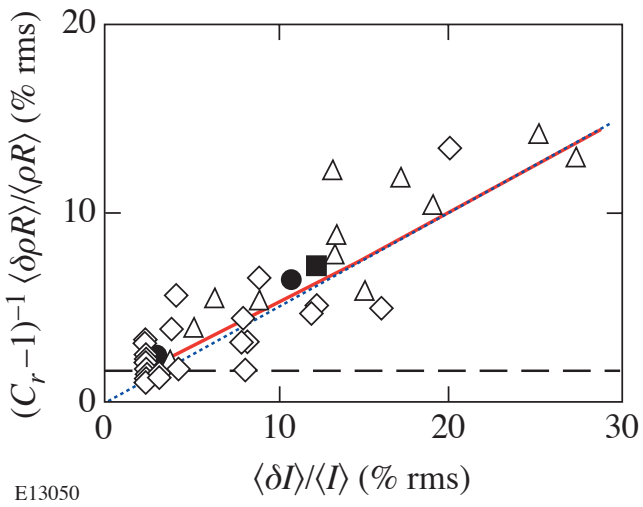

\section{Figure 98.8}

Plot of $y \equiv\left(C_{r}-1\right)^{-1}\langle\delta \rho R\rangle /\langle\rho R\rangle$ versus $x \equiv\langle\delta I\rangle /\langle I\rangle$ for the shots described in the text. The solid line represents a least-squares fit of the data to the function $y(x)=\sqrt{A^{2}+B^{2} x^{2}}$, where $A=1.63 \pm 0.33$ and $B=0.50 \pm 0.03$; the reduced $\chi^{2}$ was 1.24. The dotted line represents the contribution of $\langle\delta I\rangle /\langle I\rangle$, while the dashed line represents the mean contribution of all other sources of asymmetry. Open diamonds correspond to room-temperature capsules with plastic shells, while triangles correspond to cryogenic capsules; it is notable that the two types of data are fairly consistent with each other. Taken separately, the plastic-shell data give $B=0.41 \pm 0.05$ and the cryogenic data give $B=0.55 \pm 0.04$. In most cases, values of $\langle\delta I\rangle /\langle I\rangle>3 \%$ were due to offsets of capsules from the target chamber center. Solid circles $(20-\mu \mathrm{m} \mathrm{CH}$ shell) and a solid square (26- $\mu \mathrm{m} \mathrm{CH}$ shell) are from 2-D simulations and show good agreement with the data and with Eq. (3). beams turned off, where $\langle\delta I\rangle /\langle I\rangle$ was also dominated by low$\ell$ structure. $B$ may be somewhat smaller for higher modes due to the effects of lateral mass flow; this will be the subject of future work. The data used here correspond to capsules with similar payload masses, but the derivation of Eq. (2) indicates that (logarithmic) dependence on payload mass should be very weak. ${ }^{1}$ In addition, only one fill pressure was used in the roomtemperature capsules (18 atm), but data from other experiments ${ }^{11}$ with the much lower fill pressure of 4 atm are consistent with Eq. (3) (although all of these data fall in the low $\langle\delta I\rangle /\langle I\rangle$, low $\langle\delta \rho R\rangle /\langle\rho R\rangle$ grouping of Fig. 98.8). Of particular interest is the fact that Eq. (3) seems equally valid for both roomtemperature, $\mathrm{CH}$-shell capsules and cryogenic capsules, even though these two types of capsules have very different theoretical susceptibilities to the RT instability. ${ }^{1}$ The convergencedriven growth is probably more important than RT effects for the low modes under study here. (High-mode-number RT instabilities do have indirect effects on the growth of these low modes because they cause fuel-shell mix, which decreases $C_{r}$ and thereby decreases the growth of $\delta \rho R$.) This is particularly important for lower fill pressures, where $C_{r}$ would be expected to be larger but is not; experiments show that $C_{r}$ is nearly the same for 4-atm capsules as for 18-atm capsules. ${ }^{25}$ The data demonstrate that the growth of these low-mode asymmetries is driven primarily by convergence. Whereas different pulse shapes (adiabats), drive energies, or payload masses may result in different asymmetry amplitudes, the primary differences are likely to be due to the size of $C_{r}$ rather than the size of the coefficient $1 / 2$ in Eq. (3) or even the breakdown of the scaling itself. We conclude that the coefficient $1 / 2$ in Eq. (3) may be slightly different in different ablative-drive contexts, but probably not by much.

Other evidence supports the scaling of Eq. (3). As shown in Fig. 98.8, it is compatible with 2-D simulations for two different shell thicknesses (20 and $26 \mu \mathrm{m}$ ). This shows that the 1-D arguments used to derive Eq. (3), and also the arguments given for the weak dependence on payload mass, are compatible with 2-D simulations. The weak dependence on payload mass is experimentally shown through comparisons of the data shown here with recent results with $26-\mu \mathrm{m}$-shell capsules, ${ }^{26}$ which also indicates that the scaling applies at all angles, applies for modes $\ell=1$ and $\ell=2$ individually, and applies at separate times during the implosion.

The scaling of Eq. (3) is useful for estimating behavior in future experiments. On OMEGA, the performance of cryogenic implosions has been shown to be diminished when target offsets cause low-mode asymmetries. ${ }^{21,22}$ Another example is 
cone-capsule, fast-ignition experiments on OMEGA, ${ }^{27}$ where there is no laser illumination on part of the capsule (essentially an $\ell=1$ mode). For future indirect-drive experiments at the NIF, low-mode symmetry is an important design issue. ${ }^{1}$ Since very high values of $C_{r}$ ( 30 to 40$)$ are required for ignition, ${ }^{1}$ Eq. (3) implies that even small amounts of drive asymmetry can disrupt implosion dynamics. Although the experimental data used in this article are from direct-drive implosions, the scaling for indirect drive should theoretically be similar because the drive pressure scales with $I$ in a similar way; ${ }^{1}$ direct experimental evidence is currently being sought in ongoing indirect-drive experiments at OMEGA. ${ }^{28}$ Another NIF illumination scheme under consideration is polar direct drive (PDD), ${ }^{29}$ in which laser beams arranged in a six-ring configuration normally used for indirect drive will be used for direct drive; although this configuration will be optimized as much as possible, it will involve significant low-mode illumination asymmetry, and it is important to know how serious that will be for implosion performance. Equation (3) can be used in these cases to estimate constraints on $\langle\delta I\rangle /\langle I\rangle$ if we know the upper limit of $\langle\delta \rho R\rangle /\langle\rho R\rangle$ that an imploded capsule can tolerate, although in some cases the criteria for ignition have been stated in terms of the symmetry of the hot, compressed core ${ }^{1}$ rather than the symmetry of total $\rho R$. Current work is underway to study the relationship between core symmetry and total $\rho R$ symmetry in indirect-drive implosions. ${ }^{28}$

In summary, we have performed the first experiments to systematically study $\rho R$ asymmetries and their relationship with laser illumination asymmetries for direct-drive capsule implosions on OMEGA. A scaling law relating $\langle\delta \rho R\rangle /\langle\rho R\rangle$ to $\langle\delta I\rangle /\langle I\rangle$ has been found, and it has critical implications for future work on the National Ignition Facility (NIF) as well as on OMEGA.

\section{ACKNOWLEDGMENT}

This work was supported in part by the U.S. Department of Energy Contract \#DE-FG03-99SF21782, LLE subcontract \#PO410025G, LLNL subcontract \#B313975, the U.S. Department of Energy Office of Inertial Confinement Fusion under Cooperative Agreement No. DE-FC03-92SF19460, and the New York State Energy Research and Development Authority.

\section{REFERENCES}

1. J. D. Lindl, Phys. Plasmas 2, 3933 (1995).

2. S. W. Haan et al., Phys. Plasmas 2, 2480 (1995).

3. S. E. Bodner, D. G. Colombant, J. H. Gardner, R. H. Lehmberg, S. P. Obenschain, L. Phillips, A. J. Schmitt, J. D. Sethian, R. L. McCrory, W. Seka, C. P. Verdon, J. P. Knauer, B. B. Afeyan, and H. T. Powell, Phys. Plasmas 5, 1901 (1998).
4. R. L. McCrory, R. E. Bahr, R. Betti, T. R. Boehly, T. J. B. Collins, R. S. Craxton, J. A. Delettrez, W. R. Donaldson, R. Epstein, J. Frenje, V. Yu. Glebov, V. N. Goncharov, O. Gotchev, R. Q. Gram, D. R. Harding, D. G. Hicks, P. A. Jaanimagi, R. L. Keck, J. Kelly, J. P. Knauer, C. K. Li, S. J. Loucks, L. D. Lund, F. J. Marshall, P. W. McKenty, D. D. Meyerhofer, S. F. B. Morse, R. D. Petrasso, P. B. Radha, S. P. Regan, S. Roberts, F. Séguin, W. Seka, S. Skupsky, V. Smalyuk, C. Sorce, J. M. Soures, C. Stoeck1, R. P. J. Town, M. D. Wittman, B. Yaakobi, and J. D. Zuegel, Nucl. Fusion 41, 1413 (2001).

5. D. D. Meyerhofer, J. A. Delettrez, R. Epstein, V. Yu. Glebov, V. N. Goncharov, R. L. Keck, R. L. McCrory, P. W. McKenty, F. J. Marshall, P. B. Radha, S. P. Regan, S. Roberts, W. Seka, S. Skupsky, V. A. Smalyuk, C. Sorce, C. Stoeckl, J. M. Soures, R. P. J. Town, B. Yaakobi, J. D. Zuegel, J. Frenje, C. K. Li, R. D. Petrasso, D. G. Hicks, F. H. Séguin, K. Fletcher, S. Padalino, M. R. Freeman, N. Izumi, R. Lerche, T. W. Phillips, and T. C. Sangster, Phys. Plasmas 8, 2251 (2001).

6. S. W. Haan, Phys. Rev. A 39, 5812 (1989).

7. M. S. Plesset, J. Appl. Phys. 25, 96 (1954).

8. R. D. Petrasso, C. K. Li, M. D. Cable, S. M. Pollaine, S. W. Haan, T. P. Bernat, J. D. Kilkenny, S. Cremer, J. P. Knauer, C. P. Verdon, and R. L. Kremens, Phys. Rev. Lett. 77, 2718 (1996).

9. B. Yaakobi, V. A. Smalyuk, J. A. Delettrez, F. J. Marshall, D. D. Meyerhofer, and W. Seka, Phys. Plasmas 7, 3727 (2000).

10. F. H. Séguin, C. K. Li, D. G. Hicks, J. A. Frenje, K. M. Green, R. D. Petrasso, J. M. Soures, D. D. Meyerhofer, V. Yu. Glebov, C. Stoeck1, P. B. Radha, S. Roberts, C. Sorce, T. C. Sangster, M. D. Cable, S. Padalino, and K. Fletcher, Phys. Plasmas 9, 2725 (2002); F. H. Séguin, C. K. Li, J. A. Frenje, S. Kurebayashi, R. D. Petrasso, F. J. Marshall, D. D. Meyerhofer, J. M. Soures, T. C. Sangster, C. Stoeck1, J. A. Delettrez, P. B. Radha, V. A. Smalyuk, and S. Roberts, Phys. Plasmas 9, 3558 (2002).

11. C. K. Li, F. H. Séguin, J. A. Frenje, R. D. Petrasso, R. Rygg, S. Kurebayashi, B. Schwartz, R. L. Keck, J. A. Delettrez, J. M. Soures, P. W. McKenty, V. N. Goncharov, J. P. Knauer, F. J. Marshall, D. D. Meyerhofer, P. B. Radha, S. P. Regan, T. C. Sangster, W. Seka, and C. Stoeckl, Phys. Plasmas 10, 1919 (2003).

12. T. R. Boehly, D. L. Brown, R. S. Craxton, R. L. Keck, J. P. Knauer, J. H. Kelly, T. J. Kessler, S. A. Kumpan, S. J. Loucks, S. A. Letzring, F. J. Marshall, R. L. McCrory, S. F. B. Morse, W. Seka, J. M. Soures, and C. P. Verdon, Opt. Commun. 133, 495 (1997).

13. F. H. Séguin, J. A. Frenje, C. K. Li, D. G. Hicks, S. Kurebayashi, J. R. Rygg, B.-E. Schwartz, R. D. Petrasso, S. Roberts, J. M. Soures, D. D. Meyerhofer, T. C. Sangster, J. P. Knauer, C. Sorce, V. Yu. Glebov, C. Stoeckl, T. W. Phillips, R. J. Leeper, K. Fletcher, and S. Padalino, Rev. Sci. Instrum. 74, 975 (2003).

14. This is because there is often a great deal of similarity in the asymmetries of contiguous shots, while capsule imperfections would be expected to vary randomly from shot to shot.

15. We have ignored the fact that the individual laser beams may have slightly different time histories, resulting in a small time-dependent power imbalance, as discussed in Ref. 5. 
16. F. J. Marshall, J. A. Delettrez, R. Epstein, R. Forties, R. L. Keck, J. H. Kelly, P. W. McKenty, S. P. Regan, and L. J. Waxer, Phys. Plasmas 11, 251 (2004).

17. M. H. Emery et al., Phys. Rev. Lett. 48, 253 (1982).

18. J. Delettrez, D. K. Bradley, and C. P. Verdon, Phys. Plasmas 1, 2342 (1994).

19. W. M. Manheimer, D. G. Colombant, and J. H. Gardner, Phys. Fluids 25, 1644 (1982).

20. B. Yaakobi, T. Boehly, P. Bourke, Y. Conturie, R. S. Craxton, J. Delettrez, J. M. Forsyth, R. D. Frankel, L. M. Goldman, R. L. McCrory, M. C. Richardson, W. Seka, D. Shvarts, and J. M. Soures, Opt. Commun. 39, 175 (1981).

21. T. C. Sangster, J. A. Delettrez, R. Epstein, V. Yu. Glebov, V. N. Goncharov, D. R. Harding, J. P. Knauer, R. L. Keck, J. D. Kilkenny, S. J. Loucks, L. D. Lund, R. L. McCrory, P. W. McKenty, F. J. Marshall, D. D. Meyerhofer, S. F. B. Morse, S. P. Regan, P. B. Radha, S. Roberts, W. Seka, S. Skupsky, V. A. Smalyuk, C. Sorce, J. M. Soures, C. Stoeckl, K. Thorp, J. A. Frenje, C. K. Li, R. D. Petrasso, F. H. Séguin, K. A. Fletcher, S. Padalino, C. Freeman, N. Izumi, J. A. Koch, R. A. Lerche, M. J. Moran, T. W. Phillips, and G. J. Schmid, Phys. Plasmas 10, 1937 (2003).

22. P. W. McKenty, T. C. Sangster, M. Alexander, R. Betti, R. S. Craxton, J. A. Delettrez, L. Elasky, R. Epstein, A. Frank, V. Yu. Glebov, V. N. Goncharov, D. R. Harding, S. Jin, J. P. Knauer, R. L. Keck, S. J. Loucks, L. D. Lund, R. L. McCrory, F. J. Marshall, D. D. Meyerhofer, S. P. Regan, P. B. Radha, S. Roberts, W. Seka, S. Skupsky, V. A. Smalyuk, J. M. Soures, K. A. Thorp, M. Wozniak, J. A. Frenje, C. K. Li, R. D. Petrasso, F. H. Séguin, K. A. Fletcher, S. Padalino, C. Freeman, N. Izumi, J. A. Koch, R. A. Lerche, M. J. Moran, T. W. Phillips, G. J. Schmid, and C. Sorce, "Direct-Drive Cryogenic Target Implosion Performance on OMEGA," to be published in Physics of Plasmas.
23. C. K. Li, D. G. Hicks, F. H. Séguin, J. A. Frenje, R. D. Petrasso, J. M Soures, P. B. Radha, V. Yu. Glebov, C. Stoeckl, D. R. Harding, J. P. Knauer, R. L. Kremens, F. J. Marshall, D. D. Meyerhofer, S. Skupsky, S. Roberts, C. Sorce, T. C. Sangster, T. W. Phillips, M. D. Cable, and R. J. Leeper, Phys. Plasmas 7, 2578 (2000).

24. C. K. Li and R. D. Petrasso, Phys. Rev. Lett. 70, 3059 (1993).

25. C. K. Li, F. H. Séguin, J. A. Frenje, S. Kurebayashi, R. D. Petrasso, D. D. Meyerhofer, J. M. Soures, J. A. Delettrez, V. Yu. Glebov, P. B. Radha, F. J. Marshall, S. P. Regan, S. Roberts, T. C. Sangster, and C. Stoeckl, Phys. Rev. Lett. 89, 165002 (2002)

26. F. H. Séguin, R. D. Petrasso, J. A. Frenje, C. K. Li, J. R. Rygg, C. Stoeckl, P. B. Radha, J. A. Delettrez, V. Yu. Glebov, D. D. Meyerhofer, T. C. Sangster, and J. M. Soures, Bull. Am. Phys. Soc. 47, 144 (2002); F. H. Séguin, J. R. Rygg, J. A. Frenje, C. K. Li, R. D. Petrasso, J. A. Delettrez, V. Yu. Glebov, V. N. Goncharov, R. L. Keck, J. P. Knauer, F. J. Marshall, P. W. McKenty, D. D. Meyerhofer, T. C. Sangster, V. A. Smalyuk, J. M. Soures, and S. P. Hatchett, Bull. Am. Phys. Soc. 48, 57 (2003).

27. C. Stoeckl, J. A. Delettrez, T. C. Sangster, R. B. Stephens, S. P. Hatchett, J. A. Frenje, C. K. Li, F. H. Séguin, S. Fujioka, H. Shiraga, and K. A. Tanaka, Bull. Am. Phys. Soc. 48, 298 (2003).

28. C. K. Li et al., Bull. Am. Phys. Soc. 48, 57 (2003).

29. S. Skupsky, J. A. Marozas, R. S. Craxton, R. Betti, T. J. B. Collins, J. A. Delettrez, V. N. Goncharov, P. W. McKenty, P. B. Radha, T. R. Boehly, J. P. Knauer, F. J. Marshall, D. R. Harding, J. D. Kilkenny, D. D. Meyerhofer, T. C. Sangster, and R. L. McCrory, "Polar Direct Drive on the National Ignition Facility," to be published in Physics of Plasmas. 


\section{Convective Stimulated Brillouin Scattering (SBS) in One- and Two-Ion Plasmas}

\section{Introduction}

Stimulated brillouin scattering (SBS) is the decay of an incident (pump) light wave into a frequency-downshifted (Stokes) light wave and an ion-acoustic (sound) wave. ${ }^{1}$ It is important in inertial confinement fusion (ICF) experiments because it scatters laser light away from the target, which reduces the laser energy that is available to drive the compression of the nuclear fuel. ${ }^{2}$

Unabated SBS results in a complete transfer of energy from the pump wave to the Stokes (and sound) wave(s); however, such a transfer is not observed in experiments. Several mechanisms can saturate SBS: The first and most important one is the Landau damping ${ }^{3}$ of sound waves. This is a linear phenomenon and, hence, can be significant even for small sound-wave amplitudes. Heikkinen, ${ }^{4}$ Rozmus, ${ }^{5}$ and Cohen ${ }^{6}$ have discussed a wide variety of ion-acoustic nonlinearities. Hydrodynamic effects such as daughter-wave generation and nonlinear phase shifts can limit SBS significantly if the sound-wave amplitude is large enough. Finally, if the Stokes intensity becomes comparable to the pump intensity, the effects of pump depletion become significant. Our goal is to compare these processes in one- and two-ion plasmas. Our model of sound waves consists of mass and momentum conservation equations for the ion fluids (two of each for two-ion plasmas), together with the inertionless electron-fluid equation and the Poisson equation. The results of kinetic theory ${ }^{7-9}$ were used to calculate phenomenological damping coefficients. Beating the pump with Stokes waves creates a low-frequency ponderomotive force that drives the sound wave. In this article the exact partial differential equation for a light wave is not solved; instead, an approximate ordinary differential equation is used for the amplitude of the ponderomotive force. Neglecting the transient dynamics of the ponderomotive force is a good approximation because the group velocity of light is much higher than the group velocity of sound.

A one-dimensional model is used to simulate backward scattering. The first part of this article is devoted to SBS in a one-ion plasma. In this case it is easier to understand underly- ing physical processes and to find an approximate analytical solution of the model equations. A numerical solution was obtained by a code developed at LLE. To verify numerical results, the model equations were linearized, the exact dispersion equation was found, and it was solved numerically. The solution obtained through this method was compared to a numerical solution of linearized equations at different values of pump intensities and damping coefficients. The next step was to solve numerically the nonlinear equation for ponderomotive force along with the linear ion-fluid equation. That allowed us to quantify the effects of pump depletion. The latter solution was then compared to a solution of an exact nonlinear set of equations, which showed the effect of ion-acoustic nonlinearities. The same steps were repeated to simulate SBS in two-ion plasmas. The effects of Landau damping, pump depletion, and ion-acoustic nonlinearities are shown separately for fast and slow sound waves. SBS was simulated for carbon and hydrocarbon plasmas with parameters typical for experiments on the OMEGA laser system.

\section{SBS in One-Ion Plasmas}

\section{Model Equations}

The equations governing the ion-fluid motion are similar to the equations for undriven sound waves. ${ }^{10}$ Specifically, each ion species is governed by a continuity equation

$$
\partial_{t} n_{i}+\partial_{x}\left(n_{i} v_{i}\right)=0
$$

and a momentum equation

$$
m_{i} n_{i}\left(\partial_{t}+v_{i} \partial_{x}\right) v_{i}=-Z e n_{i} \partial_{x} \phi-\partial_{x} p_{i}
$$

where $n_{i}$ is the ion (number) density, $v_{i}$ is the ion velocity, $m_{i}$ is the ion mass, $Z e$ is the ion charge, $p_{i}$ is the ion pressure, and $\phi$ is the electrostatic potential. One can use an adiabatic equation of state for ions under the assumption that the ionacoustic wave phase velocity is much larger than the ion thermal velocity. It follows from the adiabatic equation of state 
that

$$
\partial_{x} p_{i}=3 n_{i 0} T_{i}\left(n_{i} / n_{i 0}\right)^{2} \partial_{x}\left(n_{i} / n_{i 0}\right)
$$

where $T_{i 0}$ is the equilibrium ion temperature and $n_{i 0}$ is the equilibrium ion density. By substituting Eq. (3) into Eq. (2), one finds that

$$
\begin{aligned}
& m_{i} n_{i}\left(\partial_{t}+v_{i} \partial_{x}\right) v_{i} \\
& =-Z e n_{i} \partial_{x} \phi-3 n_{i 0} T_{i 0}\left(n_{i} / n_{i 0}\right)^{2} \partial_{x}\left(n_{i} / n_{i 0}\right) .
\end{aligned}
$$

The electrostatic potential is governed by the Poisson equation

$$
\partial_{x x} \phi=4 \pi e\left(n_{e}-Z n_{i}\right)
$$

With the ponderomotive term added, ${ }^{1}$ the electron-fluid momentum equation is

$$
\partial_{t} v_{e}=e \partial_{x} \phi / m_{e}-\partial_{x}\left(e a_{\perp} / m_{e} c\right)^{2} / 2-\partial_{e} p_{e} / n_{e} m_{e}
$$

where $a_{\perp}$ is the vector potential of a light wave. Because the electron thermal velocity is much higher than the phase velocity of the sound waves, one can use the isothermal equation of state

$$
p_{e}=n_{e} T_{e}
$$

for the electron fluid. By substituting Eq. (7) into Eq. (6) and neglecting electron inertia, one finds that

$$
\partial_{x} n_{e} / n_{e}=e \partial_{x} \phi / T_{e}-e \partial_{x} d / T_{e}
$$

where the ponderomotive potential $d=a_{\perp}^{2} e / 2 m_{e} c^{2}$. The integral of Eq. (8) is

$$
n_{e}=n_{e 0} \exp \left(e \phi / T_{e}-e d / T_{e}\right)
$$

where $n_{e 0}$ is equilibrium electron density. One can simplify the manipulation of equations by rewriting them in dimensionless variables. It is clear from Eq. (9) that one should measure the potentials in units of $T_{e} / e$ and the electron density in units of $n_{e 0}$. It is clear from Eq. (4) that one should measure the ion density in units of $n_{i 0}$ (equilibrium ion density). It follows from Eq. (5) and the normalization of the potentials that one should measure distance $(x)$ in units of the electron Debye length $\lambda_{\mathrm{De}}=\left(T_{e} / 4 \pi n_{e 0} e^{2}\right)^{1 / 2}$. If one measures time $(t)$ in units of the inverse ion-plasma frequency $\omega_{\mathrm{pi}}^{-1}=\left(m_{i} / 4 \pi Z n_{e 0} e^{2}\right)^{1 / 2}$, the corresponding speed unit is the ion-sound speed $c_{s}=\left(Z T_{e} / m_{i}\right)^{1 / 2}$. By making these changes in Eqs. (1), (4), (5), and (9), one obtains the dimensionless equations

$$
\partial_{t} N_{i}+\partial_{x}\left(N_{i} V_{i}\right)=0
$$

$$
\left(\partial_{t}+V_{i} \partial_{x}\right) V_{i}+\partial_{x} \Phi+\theta N_{i} \partial_{x} N_{i}=0
$$

$$
\partial_{x x}^{2} \Phi+N_{i}-N_{e}=0
$$

$$
N_{e}-\exp (\Phi-D)=0
$$

where $\theta=3 T_{i} / Z T_{e}$. The dimensionless ponderomotive potential is given by

$$
D=A_{\perp}^{2}
$$

where $A_{\perp}=e a_{\perp} /\left(2 m_{e} T_{e} c^{2}\right)^{1 / 2}$ is the dimensionless vector potential of a light wave. Physically $A_{\perp}$ is the transverse electron quiver velocity divided by the electron thermal velocity. Equations (10)-(13) describe a sound wave in plasma. The equation for the vector potential of a light wave in a plasma was derived from the Maxwell equations: ${ }^{1}$

$$
\left(\partial_{t t}^{2}-c^{2} \partial_{x x}^{2}\right) A_{\perp}=-4 \pi e^{2} n_{e} A_{\perp} / m_{e}
$$

The vector potential $A_{\perp}$ can be written as

$$
A_{\perp}=\left[A \exp \left(-i \omega_{0} t\right)+\text { c.c. }\right] / 2
$$

where $A$ is the slowly varying amplitude and $\omega_{0}$ is the pump frequency. Slowly varying amplitude $A$ satisfies ${ }^{5}$ 


$$
\begin{aligned}
& 2 i\left(n_{c} Z m_{e} / n_{e 0} m_{i}\right)^{1 / 2} \partial_{t} A \\
& +c^{2} m_{e} / T_{e} \partial_{x x}^{2} A+n_{c} / n_{e 0} a=A n_{e} / n_{e 0},
\end{aligned}
$$

where $n_{c}=m_{e} \omega_{0}^{2} /\left(4 \pi e^{2}\right)$ is the critical density. The slowly varying amplitude can be expressed as the sum of two components:

$$
A=A_{0} \exp \left(i k_{0} x\right)+A_{1} \exp \left(i k_{1} x-i \omega_{1} t\right)
$$

where $A_{0(1)}$ is the pump (Stokes)-wave amplitude, $k_{0(1)}$ is the pump (Stokes)-wave number, and $\omega_{1}$ is the frequency difference between the pump and Stokes waves. If the SBS gain length is large compared to the wavelengths of pump and Stokes waves, the envelope approximation is valid. By substituting Eq. (18) into Eq. (17) and using envelope approximation, one finds that

$$
\begin{aligned}
& \left(\partial_{t} A_{0} / v_{0}+\partial_{x} A_{0}\right) \exp \left(i k_{0} x\right) \\
& =-i \gamma A_{1} \overline{N_{e}} \exp \left[i\left(k_{1}+k_{2}\right) x-i\left(\omega_{1}+\omega_{2}\right) t\right] / 2, \\
& \left(-\partial_{t} A_{1}^{*} / v_{0}+\partial_{x} A_{1}^{*}\right) \exp \left(-i k_{1} x+i \omega_{1} t\right) \\
& =-i \gamma A_{0}^{*} \overline{N_{e}} \exp \left[i\left(-k_{0}+k_{2}\right) x-i \omega_{2} t\right] / 2
\end{aligned}
$$

where $\overline{N_{e}}$ is the first Fourier harmonic of the electron-density fluctuations, $v_{0}$ is the group velocity of the pump wave, $k_{2}\left(\omega_{2}\right)$ is the wave number (frequency) of the undriven sound wave, and the coupling constant $\gamma=T_{e} /\left(k_{2} m_{e} c^{2}\right)$. In the context of SBS, the plasma response is important only near resonance. For three-wave processes the resonance conditions are

$$
\begin{gathered}
\omega_{1}+\omega_{2}=0, \\
k_{0}-k_{1}-k_{2}=0 .
\end{gathered}
$$

The undriven sound-wave frequency $\omega_{2}$ and wave number $k_{2}$ satisfy the dispersion relation ${ }^{10}$

$$
\omega_{2}=k_{2}\left[\left(1+k_{2}^{2}\right)+\theta\right]^{1 / 2} .
$$

By using Eqs. (21) and (22), one can reduce Eqs. (19) and (20) to

$$
\begin{gathered}
\partial_{t} A_{0} / v_{0}+\partial_{x} A_{0}=-i \gamma A_{1} \overline{N_{e}} / 2, \\
-\partial_{t} A_{1}^{*} / v_{0}+\partial_{x} A_{1}^{*}=-i \gamma A_{0}^{*} \overline{N_{e}} / 2 .
\end{gathered}
$$

Because the light-wave group velocity is much higher than the sound-wave group velocity, the intrinsic relaxation time is much shorter than the SBS gain time. Consequently, the time derivatives in Eqs. (24) and (25) can be neglected, in which case the equations reduce to

$$
\partial_{x} A_{0}=-i \gamma A_{1} \overline{N_{e}} / 2
$$

$$
\partial_{x} A_{1}^{*}=-i \gamma A_{0}^{*} \overline{N_{e}} / 2 .
$$

By substituting Eq. (16) into Eq. (14) one finds that

$$
D=\left[A^{2} \exp \left(-2 i \omega_{0} t\right)+A^{* 2} \exp \left(2 i \omega_{0} t\right)+2|A|^{2}\right] / 4 .
$$

The first two terms on the right-hand side of Eq. (28) are nonresonant and can be omitted, thus one obtains

$$
D=|A|^{2} / 2 .
$$

By substituting Eq. (18) into Eq. (29) and neglecting nonresonant terms, one finds that

$$
D=\left[A_{0} A_{1}^{*} \exp \left(i k_{2} x-i \omega_{2} t\right)+\text { c.c. }\right] / 2 .
$$

It follows from Eq. (30) that the Fourier harmonic of the ponderomotive potential is

$$
\bar{D}=A_{0} A_{1}^{*} .
$$


By differentiating Eq. (31) and substituting $\partial_{x} A_{0,1}$ from Eqs. (26) and (27), one obtains

$$
\partial_{x} \bar{D}=-i \gamma \overline{N_{e}}\left(4|\bar{D}|^{2}+C\right)^{1 / 2} / 2
$$

where the integration constant $C=\left(\left|A_{0}\right|^{2}+\left|A_{1}\right|^{2}\right)^{2}-4|\bar{D}|^{2}$. One can calculate $C$ at the left boundary $(x=0)$ and express it in terms of two physical parameters: the pump intensity $I=\left|A_{0}(0)\right|^{2}$ and the reflectivity $R=\left|A_{1}(0)\right|^{2} /\left|A_{0}(0)\right|^{2}$ :

$$
C=[I(1-R)]^{2}
$$

The physical laser intensity $I_{l}=c\left(k_{0}\left|a_{0}\right|\right)^{2} /(8 \pi)$, from which it follows that the normalized intensity

$$
I=\left(I_{l} e^{2} \lambda_{0}^{2}\right) /\left(\pi m_{e} T_{e} c^{3}\right)
$$

If $I_{l}$ is measured in $\mathrm{W} / \mathrm{cm}^{2}, \lambda_{0}$ is measured in $\mu \mathrm{m}$, and $T_{e}$ is measured in $\mathrm{keV}$, then

$$
I \approx 2 \times 10^{-16} I_{l} \lambda_{0}^{2} / T_{e}
$$

By adding phenomenological damping terms to Eqs. (11) and (12) and ponderomotive potential terms to Eqs. (13) and (14), one obtains

$$
\begin{gathered}
\partial_{t} N_{i}+\partial_{x}\left(N_{i} V_{i}\right)+\xi\left(N_{i}-1\right)=0, \\
\left(\partial_{t}+V_{i} \partial_{x}\right) V_{i}+\partial_{x} \Phi+\theta N_{i} \partial_{x} N_{i}+\zeta V_{i}=0, \\
\partial_{x x}^{2} \Phi+N_{i}-\exp \left\{\Phi-\Re\left[\bar{D} \exp \left(i k_{2} x-i \omega_{2} t\right)\right]\right\}=0, \\
N_{e}-\exp \left\{\Phi-\Re\left[\bar{D} \exp \left(i k_{2} x-i \omega_{2} t\right)\right]\right\}=0,
\end{gathered}
$$

where $\mathfrak{R}(f)$ denotes the real part of $f$. Equations (35)-(38), (32), and (33) form a complete set that self-consistently describes SBS.

We used the following initial and boundary conditions: the plasma is undisturbed initially and remains so at the left boundary $(x=0)$; SBS is seeded by a finite-amplitude Stokes wave at the right boundary $(x=L)$. We used $\left|A_{1}(L)\right|^{2}=10^{-6} I$, which corresponds to

$$
|\bar{D}(L)|^{2}=10^{-3} I .
$$

\section{Numerical Scheme}

Our code uses the MacCormack (MC) method ${ }^{11}$ to solve the ion-fluid Eqs. (35) and (36), written in conservation form. MC is a two-step method: at the first step the values of the ion density and velocity are predicted using the first-order forward difference scheme associated with Eqs. (35) and (36):

$$
N_{i_{j}}^{\overline{k+1}}=N_{i_{j}}^{k}-c\left[\left(N_{i} V_{i}\right)_{j+1}^{k}-\left(N_{i} V_{i}\right)_{j}^{k}\right]-\delta t \xi\left(N_{i j}^{k}-1\right)
$$

$$
\begin{aligned}
V_{i_{j}}^{\overline{k+1}}= & V_{i_{j}}^{k}-c\left[\left(V_{i}^{2} / 2+\Phi+\theta N_{i}^{2} / 2\right)_{j+1}^{k}\right. \\
& \left.-\left(V_{i}^{2} / 2+\Phi+\theta N_{i}^{2} / 2\right)_{j}^{k}\right]-\delta t \xi V_{i_{j}}^{k} .
\end{aligned}
$$

In Eqs. (40) and (41), the subscript $j$ denotes the position $j \delta x$, the superscript $k$ denotes the time $k \delta t$, and $c=\delta t / \delta x$ is the convection number.

With $N_{i_{j}}^{\overline{k+1}}$ known, the predicted values of the electrostatic potential $\Phi_{j}^{\overline{k+1}}$, electron density $N_{e_{j}}^{\overline{k+1}}$ and ponderomotive potential $D_{j}^{\overline{k+1}}$ can be determined by solving Eqs. (32), (33), (37), and (38) iteratively. First, Eq. (37) is solved using the ponderomotive potential obtained in the previous iteration (previous time step for the first iteration). Equation (37) is solved using the Newton iteration method, which was described in detail in Ref. 10. The values of the electrostatic potential obtained from Eq. (37) are used in Eq. (38) to calculate the electron density $N_{e}$. The discrete Fourier transform $^{12}$ is used to calculate the first harmonic of $N_{e}$. Fourier transform actually gives the values of the first harmonic averaged over one wavelength; thus, Eq. (32) has to be solved on a grid whose points are separated by exactly one wavelength. Linear interpolation was used to calculate the values of ponderomotive potential between those grid points:

$$
\bar{D}(x)=\bar{D}\left(x_{l}\right)+\left[\bar{D}\left(x_{r}\right)-\bar{D}\left(x_{l}\right)\right]\left(x-x_{1}\right) / \lambda_{2},
$$


where $x_{l}$ and $x_{r}$ are left and right grid points and $\lambda_{2}$ is the sound wavelength. The square of the ponderomotive potential amplitude in Eq. (32) also has to be averaged over one wavelength: $\left\langle\bar{D}^{2}\right\rangle=\int_{x_{l}}^{x_{r}} \bar{D}(x)^{2} d x / \lambda_{2}$. By using formula (42) for $\bar{D}(x)$ and integrating, one obtains

$$
\begin{aligned}
\left\langle\bar{D}^{2}\right\rangle= & \left\{\left|\bar{D}\left(x_{l}\right)\right|^{2}+\left|\bar{D}\left(x_{r}\right)\right|^{2}+\Re\left[\bar{D}\left(x_{l}\right)\right] \Re\left[\bar{D}\left(x_{r}\right)\right]\right. \\
& \left.+\Im\left[\bar{D}\left(x_{l}\right)\right] \Im\left[\bar{D}\left(x_{r}\right)\right]\right\} / 3,
\end{aligned}
$$

where $\Im(f)$ denotes the imaginary part of $f$.

A straightforward numerical scheme is then used to solve Eq. (32):

$$
\bar{D}_{c}\left(x_{l}\right)=\bar{D}_{c}\left(x_{r}\right)+i \gamma \lambda_{2} \bar{N}_{e}\left(4\left\langle\bar{D}^{2}\right\rangle_{p}+C\right)^{1 / 2} / 2
$$

The values of $\left\langle\bar{D}^{2}\right\rangle_{p}$ and $C_{p}$ obtained in the previous iteration (previous time step for the first iteration) are used to calculate $\bar{D}_{c}$ in the current iteration. The values of $\bar{D}_{c}$ are calculated from right to left because the value of $\bar{D}$ at the right boundary is fixed [Eq. (39)]. The values of $\bar{D}_{c}$ are then used in Eq. (33) to calculate $R_{c}$ and $C_{c}$. Subsequently, Eq. (37) is solved using the values of ponderomotive potential obtained in the previous iteration. These iterations are repeated until the convergence condition

$$
\left|\left(R_{c}-R_{p}\right) / R_{p}\right|<\epsilon
$$

is satisfied. At the second step of the MC method, spatial derivatives are evaluated using the first-order forward-difference approximation based on the predicted values of the density, velocity, and electrostatic potential. Corrected values of density and velocity are obtained by averaging the spatial derivatives calculated at the first and second steps:

$$
\begin{aligned}
N_{i_{j}}^{k+1}= & N_{i_{j}}^{k}-c\left[\left(N_{i} V_{i}\right)_{j+1}^{k}-\left(N_{i} V_{i}\right)_{j}^{k}\right. \\
& \left.+\left(N_{i} V_{i}\right)_{j}^{\overline{k+1}}-\left(N_{i} V_{i}\right)_{j-1}^{\overline{k+1}}\right] / 2 \\
& -\delta t\left[\zeta\left(N_{i_{j}}^{k}-1\right)+\zeta\left(N_{i_{j}}^{\overline{k+1}}-1\right)\right] / 2,
\end{aligned}
$$

$$
\begin{aligned}
V_{i_{j}}^{k+1}= & V_{i_{j}}^{k}-c\left[\left(V_{i}^{2} / 2+\Phi+\theta N_{i}^{2} / 2\right)_{j+1}^{k}\right. \\
& -\left(V_{i}^{2} / 2+\Phi+\theta N_{i}^{2} / 2\right)_{j}^{k} \\
& +\left(V_{i}^{2} / 2+\Phi+\theta N_{i}^{2} / 2\right)_{j}^{\overline{k+1}} \\
& -\left(V_{i}^{2} / 2+\Phi+\theta N_{i}^{2} / 2\right)_{j-1}^{\overline{k+1}} / 2 \\
& -\delta t\left[\zeta V_{i_{j}}^{k}+\zeta V_{i_{j}}^{\overline{k+1}}\right] / 2 .
\end{aligned}
$$

The iterations described at the first step are repeated at the second step to calculate the corrected values of the electrostatic potential $\Phi_{j}^{k+1}$, electron density $N_{e j}^{k+1}$, and ponderomotive potential amplitude $\bar{D}_{j}^{k+1}$. The MC method is of second-order accuracy in both time and space and is conditionally stable. ${ }^{11}$ The Courant stability condition for the MC scheme applied to the sound-wave equations is derived in Ref. 10.

The sound-wave equations are solved on a spatial interval that is longer than the interaction length of the sound and light waves. To prevent the reflection of the sound wave from the right boundary, the spatial interval includes an extra region in which the sound wave is strongly damped and not driven.

\section{Linear Regime of SBS}

By linearizing Eqs. (35)-(38), one finds that

$$
\begin{gathered}
\partial_{t} N_{i}^{(1)}+\partial_{x} V_{i}^{(1)}+\zeta N_{i}^{(1)}=0, \\
\partial_{t} V_{i}^{(1)}+\partial_{x} \Phi+\theta \partial_{x} N_{i}^{(1)}+\zeta V_{i}^{(1)}=0, \\
\partial_{x x}^{2} \Phi^{(1)}+N_{i}^{(1)}-N_{e}^{(1)}=0, \\
N_{e}^{(1)}-\Phi^{(1)}+D^{(1)}=0,
\end{gathered}
$$

where $N_{i}^{(1)}=N_{i}-1, N_{e}^{(1)}=N_{e}-1, V_{i}^{(1)}=V_{i}, \Phi^{(1)}=\Phi$, and $D^{(1)}=D$. By substituting

$$
y^{(1)}=\left[\tilde{y} \exp \left(-\sigma x+i k_{2} x-i \omega_{2} t\right)+\text { c.c. }\right] \text {, }
$$


where $\sigma$ is the spatial growth rate, and differentiating, one obtains the linear equations

$$
\begin{gathered}
(i \omega-\zeta) \tilde{N}_{i}-(i k-\sigma) \tilde{V}_{i}=0 \\
(i \omega-\zeta) \tilde{V}_{i}-(i k-\sigma)\left(\tilde{\Phi}+\theta \tilde{N}_{i}\right)=0 \\
(i k-\sigma)^{2} \tilde{\Phi}+\tilde{N}_{i}-\tilde{N}_{e}=0 \\
\tilde{N}_{e}-\tilde{\Phi}+\tilde{D}=0
\end{gathered}
$$

in which $\omega$ and $k$ are the frequency and wave number of the undriven sound wave (the subscript 2 was omitted for simplicity). By solving Eqs. (52)-(55) one finds that

$$
\begin{aligned}
& \tilde{N}_{e} /(i k-\sigma)^{2} \\
& =\left\{1-1 /\left[\theta(i k-\sigma)^{2}-(i \omega-\zeta)^{2}\right]\right\}\left(\tilde{N}_{e}+\tilde{D}\right) .
\end{aligned}
$$

Linearization of the light-wave equation is equivalent to neglecting pump depletion

$$
\partial_{x} \bar{D}=-i \gamma I \overline{N_{e}} / 2
$$

One can rewrite Eq. (57) in terms of $\tilde{D}=\bar{D} \exp (-\sigma x)$ and $\tilde{N}_{e}=\bar{N}_{e} \exp (-\sigma x)$ :

$$
\tilde{D}=\tilde{N}_{e} i \gamma I /(2 \sigma)
$$

It follows from Eqs. (56) and (58) that

$$
\begin{aligned}
& 1 /(i k-\sigma)^{2} \\
& =\left\{1-1 /\left[\theta(i k-\sigma)^{2}-(i \omega-\zeta)^{2}\right]\right\}[1+i \gamma I /(2 \sigma)] .
\end{aligned}
$$

By using the sound-wave dispersion relation (23) and neglecting terms of second and higher order in $\sigma / k$ and $\delta / \omega$, one can reduce Eq. (59) to

$$
\sigma=-\gamma I k / 4 \sigma+\eta\left(c_{2} \zeta-\theta \sigma\right)
$$

where phase velocity $c_{2}=\omega / k$ and $\eta=\left(1+k^{2}\right)^{2}$. Equation (60) is a quadratic equation and can be solved analytically. The spatial growth rates are given by

$$
\sigma_{ \pm}=\frac{\eta c_{2} \zeta \pm\left[\eta^{2} c_{2}^{2} \xi^{2}-\gamma I k(1+\eta \theta)\right]^{1 / 2}}{2(1+\eta \theta)}
$$

By using boundary conditions for the ion density $N^{(1)}(0)=0$, ponderomotive potential $D(L)=D_{0} \cos (k x-\omega t)$, and Eq. (58), one finds that

$$
\begin{aligned}
D= & D_{0} \frac{\exp \left(-\sigma_{+} x\right) / \sigma_{+}-\exp \left(-\sigma_{-} x\right) / \sigma_{-}}{\exp \left(-\sigma_{+} L\right) / \sigma_{+}-\exp \left(-\sigma_{-} L\right) / \sigma_{-}} \\
& \times \cos (k x-\omega t),
\end{aligned}
$$

$$
\begin{aligned}
N_{i}= & 1+2 D_{0}\left(1+k^{2}\right) \frac{\exp \left(-\sigma_{+} x\right)-\exp \left(-\sigma_{-} x\right)}{\exp \left(-\sigma_{+} L\right) / \sigma_{+}-\exp \left(-\sigma_{-} L\right) / \sigma_{-}} \\
& \times \sin (k x-\omega t) /(\gamma I)
\end{aligned}
$$

Equation (60) has real solutions for growth rates $\sigma$ only if

$$
\xi^{2}>\gamma I k(1+\eta \theta) /\left(\eta c_{2}\right)^{2}
$$

When the ion-acoustic damping is weak, condition (64) is violated and there is no physically meaningful solution for $\sigma$. For this case the linear SBS equations predict absolute instability. ${ }^{13}$ There is no steady-state solution of linear SBS equations in this case. The only saturation mechanisms in the case of weak ion-acoustic damping are nonlinear effects such as pump depletion and hydrodynamic nonlinearities. We consider convective SBS with strong ion-acoustic damping. In this case nonlinear effects can also be important, but even in the linear 
regime SBS can be saturated by damping. For very strong damping

$$
\xi^{2}>\gamma I k
$$

By neglecting the second- and higher-order powers of $\gamma I k / \xi^{2}$ in the Maclaurin expansion of formula (61), one obtains

$$
\begin{gathered}
\sigma_{+} \approx \frac{\eta c_{2} \zeta}{1+\eta \zeta}-\frac{\gamma I k}{4 \eta c_{2} \zeta} \\
\sigma_{-} \approx \frac{\gamma I k}{4 \eta c_{2} \zeta}
\end{gathered}
$$

If condition (65) is satisfied, then $\sigma_{+}>>\sigma_{-}$and $\exp \left(-\sigma_{+} x\right)$ $<\exp \left(-\sigma_{-} x\right)$. Neglecting $\exp \left(-\sigma_{+} x\right)$, one can reduce Eq. (62) to

$$
D \approx D_{0} \exp [G(L-x)] \cos (k x-\omega t)
$$

where $G=\gamma I k / 4 \eta c_{2} \zeta$ is the well-known convective growth rate.

To assess the accuracy of the analytical approximations, we chose typical parameters and solved Eq. (59) numerically. There are up to eight solutions for $\sigma$, but only two of them have $|\mathfrak{R}(\sigma)|>>|\Im(\sigma)|$ and are relevant to SBS. For future reference we will refer to the solution obtained by the method described above as the combined solution.
We modeled SBS in a carbon plasma $(Z=6)$ with parameters that are typical of ICF experiments on the OMEGA laser system: $\lambda_{0}=0.35 \mu \mathrm{m}, I_{L}=10^{15} \mathrm{~W} / \mathrm{cm}^{2}, T_{e} / T_{i}=4, T_{e}=1 \mathrm{keV}$, and $n_{0 e} / n_{\mathrm{cr}}=0.1$. We used kinetic theory ${ }^{14}$ to evaluate the Landau damping rate

$$
\begin{aligned}
\zeta / \omega= & \frac{(\pi / 8)^{1 / 2}}{\left(1+k^{2}\right)} \\
& \times c_{s}\left[\left(\frac{m_{e} Z}{m_{i}}\right)^{1 / 2}+\left(\frac{T_{e} Z}{T_{i}}\right)^{3 / 2} \exp \left(-c_{s}^{2} \frac{T_{e} Z}{2 T_{i}}\right)\right] .
\end{aligned}
$$

For the OMEGA parameters listed above, the Landau damping rate $\zeta / \omega \approx 3.5 \%$.

The numerical (steady-state) solution of Eqs. (48)-(51) and (57) is compared to the analytical and combined solutions in Fig. 98.9. SBS in carbon plasma was simulated for the OMEGA-like parameters listed above. The three solutions agree perfectly. The reflectivity $\left(R_{N}\right)$ obtained through direct numerical solution of Eqs. (48)-(51) and $(57)=20.6 \%$. The reflectivity $\left(R_{A, C}\right)$ obtained through analytical and combined solution $=20.8 \%$. Relative difference $\left(R_{A, C}-R_{N}\right) / R_{N}=0.9 \%$.
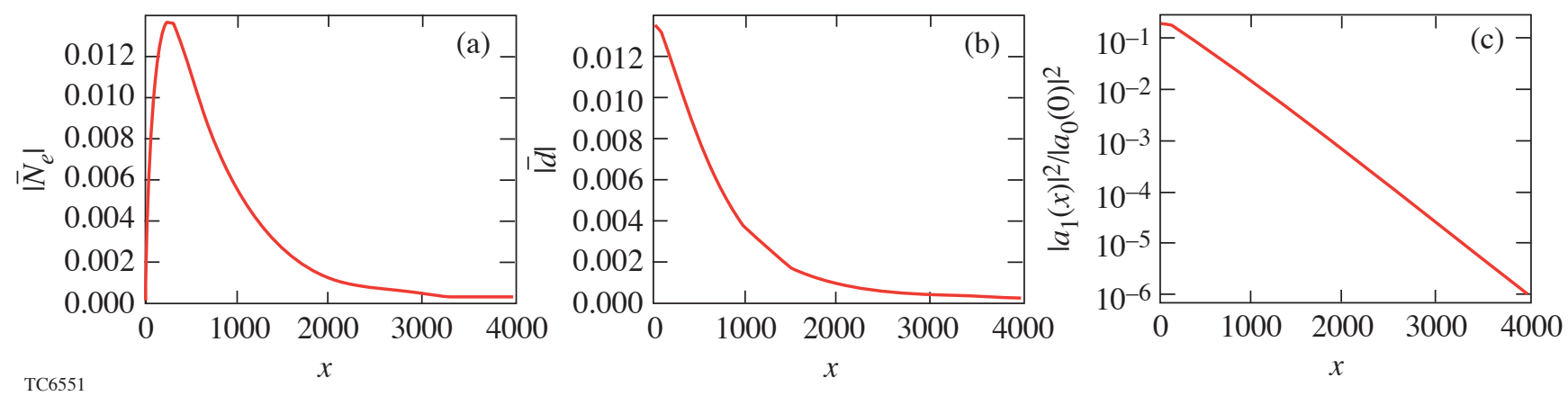

\section{Figure 98.9}

(a) First harmonic of electron density; (b) ponderomotive potential amplitude; (c) Stokes-wave intensity normalized to pump-wave intensity versus distance for steady state of SBS. Solutions of linearized equations [(48)-(51), (57)] describing SBS obtained through different methods are compared. Dotted lines represent numerical solution. Dashed lines represent combined solution obtained by numerical solution of Eq. (59). Solid lines represent analytical solution. All three solutions are indistinguishable. Simulation parameters are $\lambda_{0}=0.35 \mu \mathrm{m}, I_{L}=10^{15} \mathrm{~W} / \mathrm{cm}^{2}, T_{e} / T_{i}=4, T_{e}=1 \mathrm{keV}$, and $n_{0 e} / n_{\mathrm{cr}}=0.1$. 


\section{Nonlinear Saturation}

Convective SBS can be significantly reduced by nonlinear effects when the reflectivity is big enough. Pump depletion can be taken into account by solving the nonlinear light-wave equations (32) and (33). The effects of hydrodynamic nonlinearities can be determined by solving the nonlinear ion-fluid and Poisson equations (35)-(38). Different effects such as nonlinear phase shift ${ }^{15}$ and generation of higher-order ionwave harmonics of sound wave $e^{4,5,16}$ were studied analytically. Our goal is to compare the effects of pump depletion and hydrodynamic nonlinearities on the saturation of SBS at OMEGA-like parameters. To separate the effects of pump depletion and hydrodynamic nonlinearities we numerically solved the nonlinear light-wave equations coupled with the linear sound-wave equations (48)-(51). This partially nonlinear solution was compared to the linear solution, based on Eqs. (48)-(51) and (57), and to the numerically obtained fully nonlinear solution, based on Eqs. (32), (33), and (35)-(38). All three solutions are shown in Figs. 98.10 and 98.11. Simulation parameters are the same as those used in the previous section.

Steady-state reflectivities are as follows: linear reflectivity $R_{\mathrm{LN}}=20.6 \%$, partially nonlinear reflectivity $R_{\mathrm{PN}}=7.8 \%$, fully nonlinear reflectivity $R_{\mathrm{FN}}=6.3 \%$. Pump depletion reduces reflectivity by $60 \%$. Ion-acoustic nonlinearities reduce reflectivity by $20 \%$.

It is straightforward to express the pump intensity in terms of ponderomotive potential amplitude and constant of integration $C$. By using Eq. (31) and the definition of $C$, one finds that

$$
\left|A_{0}\right|^{2}=\left[\left(C+4|\bar{D}|^{2}\right)^{1 / 2}+C^{1 / 2}\right] / 2 .
$$

To show the effect of pump depletion we plotted the pump intensity based on the partially nonlinear solution. The pumpwave intensity normalized to the input pump intensity is shown as a function of distance in Fig. 98.12. The pump

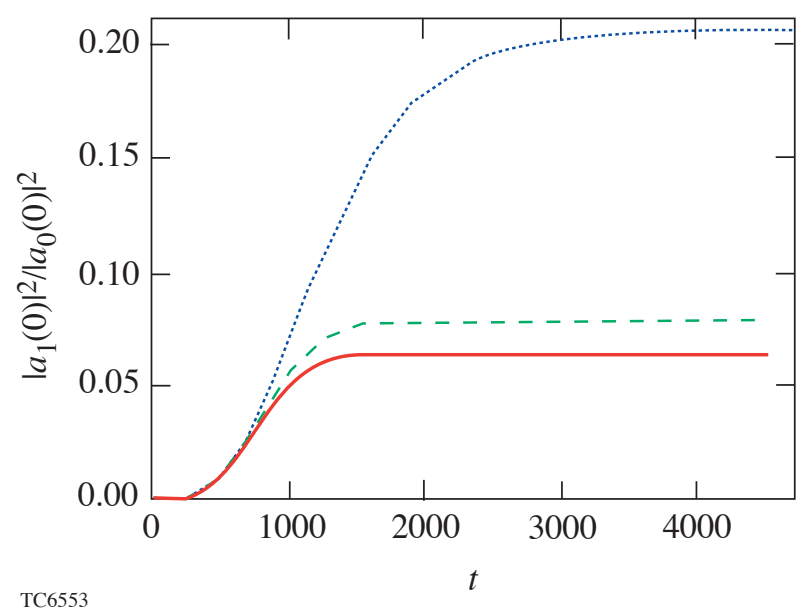

Figure 98.11

Reflectivities versus time. Dotted line represents numerical solution of linearized equations (48)-(51) and (57). Dashed line represents numerical solution of partially nonlinear equations (32), (33), and (48)-(51). Solid line represents numerical solution of fully nonlinear equations (32), (33), and (35)-(38). Simulation parameters are the same as in Fig. 98.9.
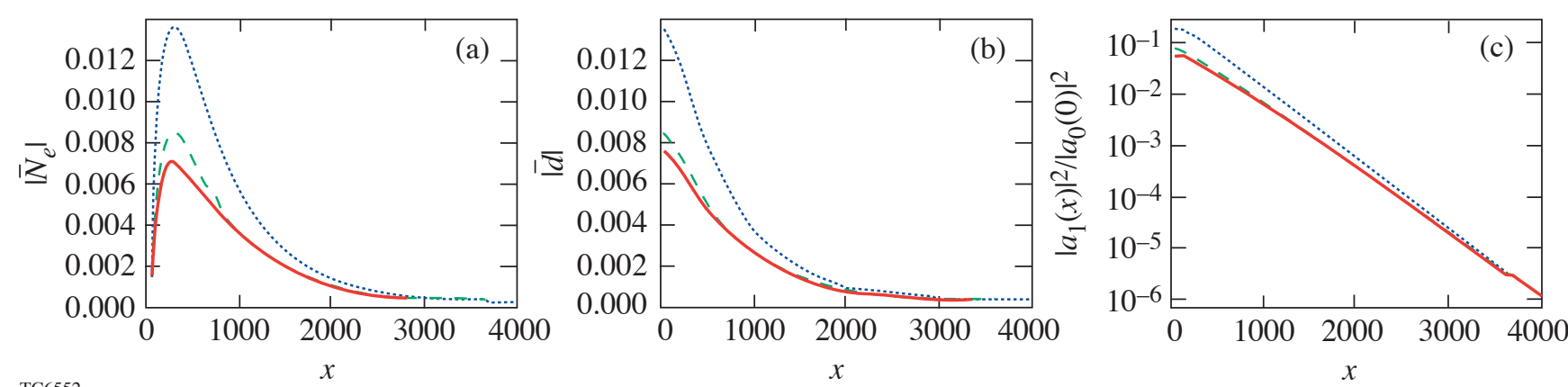

Figure 98.10

(a) First harmonic of electron density; (b) ponderomotive potential amplitude; (c) Stokes-wave intensity normalized to pump wave intensity versus distance for steady state of SBS. Dotted lines represent numerical solution of linearized equations (48)-(51) and (57). Dashed lines represent numerical solution of partially nonlinear equations (32), (33), and (48)-(51). Solid lines represent numerical solution of fully nonlinear equations (32), (33), and (35)-(38). Simulation parameters are the same as in Fig. 98.9. 
intensity decreases on a scale length of several wavelengths. As one would expect, output pump intensity

$$
\left|A_{0}(L)\right|^{2} / I=1-R_{\mathrm{PN}}+\left|A_{1}(L)\right|^{2} / I .
$$

To show the higher-order harmonic generation, we plotted the discrete Fourier spectra of the electron density near its maximum. The absolute values of the harmonic amplitudes are shown in Fig. 98.13. The second and third harmonics can have significant amplitudes, whereas the fourth and higher harmonics are negligibly small.

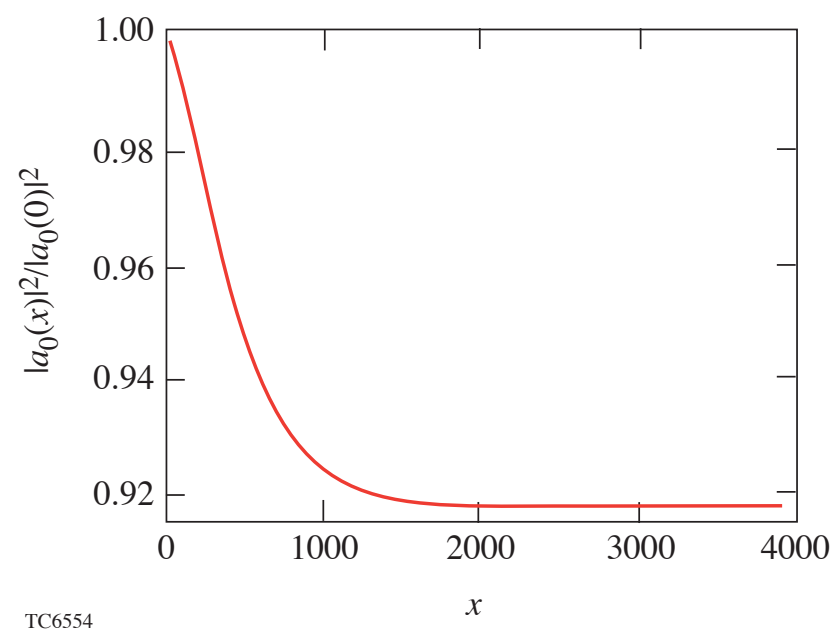

Figure 98.12

Pump-wave intensity normalized to input pump intensity versus distance for steady state of SBS. Simulation parameters are the same as in Fig. 98.9.

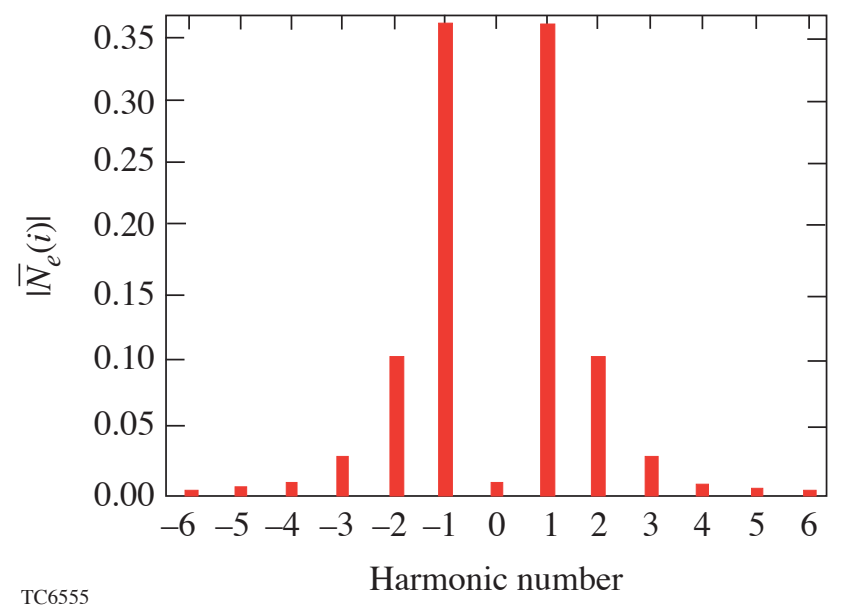

Figure 98.13

Fourier spectra of electron density near the point where it has maximal amplitude for steady state of SBS. Simulation parameters are the same as in Fig. 98.9.

\section{SBS in Two-Ion Plasmas}

\section{Model Equations}

The equations governing the ion-fluid motion are the same as those for undriven sound waves. ${ }^{17}$ For each ion species $s$, the mass and momentum conservation equations are

$$
\begin{gathered}
\partial_{t} n_{s}+\partial_{x}\left(n_{s} v_{s}\right)=0 \\
m_{s} n_{s}\left(\partial_{t}+v_{s} \partial_{x}\right) v_{s}+Z_{s} e n_{s} \partial_{x} \phi-\partial_{x} p_{s}=0,
\end{gathered}
$$

where $n_{s}$ is the ion density, $v_{s}$ is the ion velocity, $m_{s}$ is the ion mass, $Z_{s} e$ is the ion charge, $p_{s}$ is the ion pressure, and $\phi$ is the electrostatic potential. A detailed comparison of fluid and kinetic models of sound waves in two-ion plasmas is described in Ref. 9. In particular it shows that a fluid model with selfconsistent values of adiabatic exponents approximates the kinetic phase velocities of the sound waves with an accuracy close to the accuracy of the fluid model with adiabatic exponents equal to 3 for both ion species. If one assumes adiabatic exponents equal to 3 for both ion species, one finds that

$$
\partial_{x} p_{s}=3 n_{s 0} T_{s 0}\left(n_{s} / n_{s 0}\right)^{2} \partial_{x}\left(n_{s} / n_{s 0}\right)
$$

where $T_{s 0}$ is the equilibrium ion temperature and $n_{s 0}$ is the equilibrium ion density. One can easily modify Eq. (73) for a polytropic equation of state. By substituting Eq. (73) into Eq. (72), one finds that

$$
\begin{aligned}
& m_{s} n_{s}\left(\partial_{t}+v_{s} \partial_{x}\right) v_{s} \\
& +Z_{s} e n_{s} \partial_{x} \phi 3 n_{s 0} T_{s 0}\left(n_{s} / n_{s 0}\right)^{2} \partial_{x}\left(n_{s} / n_{s 0}\right)=0 .
\end{aligned}
$$

The evolution of the electrostatic potential is governed by the Poisson equation

$$
\partial_{x x} \phi=4 \pi e\left(n_{e}-\sum_{s} Z_{s} n_{s}\right) .
$$

One can simplify the manipulation of these equations by normalizing $n_{s}$ to $n_{s 0}, n_{e}$ to $n_{e 0}$, and $u_{s}$ to the sound speed of a reference species $r$. By rewriting Eqs. (71), (74), and (75) in the dimensionless form and adding phenomenological damping terms, one obtains 


$$
\begin{gathered}
\partial_{t} N_{s}+\partial_{x}\left(N_{s} V_{s}\right)+\zeta\left(N_{s}-1\right)=0 \\
\left(\partial_{t}+V_{s} \partial_{x}\right) V_{s}+\beta_{s} \partial_{x} \Phi+\theta_{s} N_{s} \partial_{x} N_{s}+\zeta V_{i}=0 \\
\partial_{x x}^{2} \Phi-N_{e}+\sum_{s} \alpha_{s} N_{s}=0
\end{gathered}
$$

where $\theta_{s}=3 T_{s} / Z_{r} T_{e} m_{r} / m_{s}, \alpha_{s}=Z_{s} n_{s 0} / n_{e 0}$ is the ion chargeto-density ratio, and $\beta_{s}=Z_{s} m_{r} / Z_{r} m_{s}$ is the ion charge-tomass ratio. Distance is measured in units of the electron-Debye length $\lambda_{\mathrm{D}}=\left(T_{e} / 4 \pi e^{2} n_{e 0}\right)^{1 / 2}$, and time is measured in units of the reference ion-plasma period $1 / \omega_{\mathrm{pi}}=\left(m_{r} / 4 \pi Z_{r} e^{2} n_{e 0}\right)^{1 / 2}$. The equations for the electron density and ponderomotive potential amplitude are the same as those for the one-ion case: Eqs. (32), (33), and (38). We used the same initial and boundary conditions as in the one-ion case.

\section{Numerical Scheme}

A modified version of the numerical scheme described in the one-ion section was used to simulate SBS in two-ion plasmas. The mass- and momentum-conservation equations for each ion species are solved to evaluate the density $N_{s}$ and velocity $V_{s}$ of each of the ion fluids. Next, the Poisson equation (78) with a weighted sum of the light- and heavy-ion charge densities is used to calculate the electrostatic potential $\Phi$. Equations (78), (33), (37), and (38) are solved using the iterative procedure described in the one-ion section. The Courant stability condition for the $\mathrm{MC}$ scheme applied to the sound-wave equation in two-ion plasmas is given in Ref. 17.

\section{Linear Regime of SBS}

By linearizing Eqs. (76)-(78), one finds that

$$
\begin{gathered}
\partial_{t} N_{s}^{(1)}+\partial_{x} V_{s}^{(1)}+\xi N_{s}^{(1)}=0, \\
\partial_{t} V_{s}^{(1)}+\beta_{s} \partial_{x} \Phi+\theta_{s} \partial_{x} N_{s}^{(1)}+\zeta V_{s}^{(1)}=0, \\
\partial_{x x}^{2} \Phi^{(1)}+\sum_{s} \alpha_{s} N_{s}^{(1)}-N_{e}^{(1)}=0 .
\end{gathered}
$$

By substituting $y^{(1)}=\left[\tilde{y} \exp \left(-\sigma x+i k_{2} x-i \omega_{2} t\right)-c . c.\right]$ into Eqs. (79)-(81) and differentiating, one obtains the linear algebraic equations

$$
\begin{gathered}
(i \omega-\zeta) \tilde{N}_{s}-(i k-\sigma) \tilde{V}_{s}=0 \\
(i \omega-\zeta) \tilde{V}_{s}-(i k-\sigma) \beta_{s}\left(\tilde{\Phi}+\theta_{s} \tilde{N}_{s}\right)=0 \\
(i k-\sigma)^{2} \tilde{\Phi}+\sum_{s} \alpha_{s} \tilde{N}_{s}-\tilde{N}_{e}=0 .
\end{gathered}
$$

The undriven sound-wave frequency $\omega$ and wave number $k$ satisfy the dispersion relation ${ }^{17}$

$$
1+k^{2}=\sum_{s} \alpha_{s} \beta_{s} k^{2} /\left(\omega^{2}-\theta_{s} k^{2}\right) .
$$

The analysis of an undriven sound wave in two-ion plasmas was described in detail in Ref. 17. Two types of sound waves exist in such plasmas: a fast wave (fa), with phase speed in the range

$$
c_{\mathrm{fa}}^{2}>\theta_{l}^{2}
$$

and a slow wave (sl), with phase speed in the range

$$
\theta_{h}^{2}<c_{\mathrm{s} 1}^{2}<\theta_{l}^{2}
$$

where the indices $l$ and $h$ denote the lighter and heavier ions, respectively. Equation (85) is biquadratic and can be solved analytically. The frequencies are given by

$$
\begin{aligned}
\omega_{\mathrm{fa}, \mathrm{sl}}^{2}= & k^{2}\left\{\mu_{l}+\theta_{l}^{2}+\mu_{h}+\theta_{h}^{2}\right. \\
& \left. \pm\left[\left(\mu_{l}+\theta_{l}^{2}-\mu_{h}-\theta_{h}^{2}\right)^{2}+4 \mu_{l} \mu_{h}\right]^{1 / 2}\right\} / 2,
\end{aligned}
$$

where $\mu_{s}=\alpha_{s} \beta_{s} /\left(1+k^{2}\right)$. In Eq. (88) the plus sign is associated with the fast wave and the minus sign is associated with the slow wave. Both sound waves can be driven and participate in SBS. We artificially separated SBS from the fast and the slow sound waves by choosing the beat frequency of ponderomotive potential. Fast- or slow-wave SBS is modeled by driving the sound wave by ponderomotive potential, which has a corresponding frequency. 
By combining Eqs. (82)-(84), (55), and (58), one finds that

$$
\begin{aligned}
& 1 /(i k-\sigma)^{2} \\
& =\left\{1-\sum_{s} \alpha_{s} \beta_{s} /\left[\theta_{s}(i k-\sigma)^{2}-(i \omega-\zeta)^{2}\right]\right\}[1+i \gamma I /(2 \sigma)] .
\end{aligned}
$$

As one should expect, the one-ion limit of Eq. (89) coincides with Eq. (59). Following the envelope-approximation procedure described in the one-ion section, we simplified Eq. (89) by substituting the undriven sound-wave frequencies given by Eq. (88) into Eq. (89) and neglecting second- and higher-order powers of $\sigma / k$ and $\xi / \omega$. The result is

$$
\sigma=-\gamma I k / 4 \sigma+\sum_{s} \alpha_{s} \beta_{s}\left(c_{2} \zeta-\theta_{s} \sigma\right) /\left(c_{2}^{2}-\theta_{s}\right)^{2}
$$

Equation (90) is quadratic and can be solved analytically. The growth rates are given by

$$
\begin{aligned}
\sigma_{1,2}= & \frac{c_{2} \zeta \sum_{s} \chi_{s}}{2\left(1+\sum_{s} \chi_{s} \theta_{s}\right)} \\
& \pm \frac{\left[\left(c_{2} \zeta \sum_{s} \chi_{s}\right)^{2}-\gamma I k\left(1+\sum_{s} \chi_{s} \theta_{s}\right)\right]^{1 / 2}}{2\left(1+\sum_{s} \chi_{s} \theta_{s}\right)},
\end{aligned}
$$

where $\chi_{s}=\alpha_{s} \beta_{s} /\left(c_{2}^{2}-\theta_{s}\right)^{2}$.

As in the one-ion case, this approximate analytical solution was compared to the exact numerical solution of Eq. (89). Just as in the one-ion case, there are up to eight numerical roots of

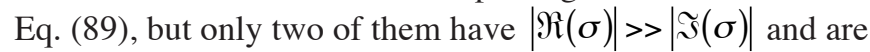
relevant to SBS. The solution obtained by the numerical solution of the analytically derived equation for $\sigma$ is also referred to as the combined solution.

The comparison of the approximate analytical solution to the exact numerical solution of Eq. (89) showed that in the case of a strong Landau damping $(\zeta / \omega \geqslant 0.1)$, neglecting secondorder powers of $\zeta / \omega$ leads to a significant error in the growthrate values.
We modeled SBS from fast and slow waves in hydrocarbon (CH) plasma $\left(\alpha_{l}=1 / 7, \alpha_{h}=6 / 7, \beta_{l}=1, \beta_{h}=1 / 2\right)$, with parameters that are typical of ICF experiments on OMEGA: $\lambda_{0}=0.35 \mu \mathrm{m}, I_{L}=2 \times 10^{15} \mathrm{~W} / \mathrm{cm}^{2}, T_{e} / T_{l}=T_{e} / T_{h}=4, T_{e}=$ $1 \mathrm{keV}, n_{0 e} / n_{\mathrm{cr}}=0.4$.

A detailed kinetic analysis of sound waves in two-ion plasmas is described in Refs. 7-9. We used the expression given in Ref. 9 for the Landau-damping rates:

$$
\begin{aligned}
\frac{\zeta_{\mathrm{fa}(\mathrm{sl})}}{\omega_{\mathrm{fa}(\mathrm{sl})}}= & c_{\mathrm{fa}(\mathrm{sl})} \frac{(\pi / 8)^{1 / 2}}{1+v+k^{2}}\left[\left(\frac{m_{e} Z_{r}}{m_{i}}\right)^{1 / 2}\right. \\
& \left.+\sum_{s} \alpha_{s} \beta_{s}\left(\frac{3}{\theta_{s}}\right)^{3 / 2} \exp \left(-\frac{3 c_{\mathrm{fa}(\mathrm{sl})}^{2}}{2 \theta_{s}}\right)\right],
\end{aligned}
$$

where $v=0$ for the fast wave and $v=3 \alpha_{l} / \theta_{l}$ for the slow wave. Coefficient $v$ reflects the reduction of the slow-wave damping rate by the light-ion Debye-screening factor. For the parameters listed above, the light-ion contribution to the Landau-damping rate $\left(\xi_{l}\right)$ dominates for both fast and slow waves and $\zeta_{l} / \omega \approx 0.13$. The heavy-ion contribution for the fast wave is negligibly small $\left(\zeta_{h} / \omega \approx 10^{-6}\right)$ because the phase velocity of the fast wave is much closer to the thermal velocity of the light ions than to the thermal velocity of the heavy ions. The phase velocity of the slow wave lies between the thermal velocities of the light and heavy ions. For the stated parameters, the heavy-ion contribution to the slow-wave Landaudamping rate is significant $\left(\zeta_{h} / \omega \approx 0.013\right)$, but smaller than the light-ion contribution. The electron contribution $\left(\zeta_{e}\right)$ is significant for both waves. For the fast wave $\zeta_{e} / \omega \approx 0.01$, whereas for the slow wave $\zeta_{e} / \omega \approx 0.005$. The relative damping rates of fast and slow waves turn out to be close $\left(\xi_{\mathrm{fa}} / \omega_{\mathrm{fa}} \approx 0.138, \zeta_{\mathrm{sl}} / \omega_{\mathrm{sl}} \approx 0.148\right)$. By definition the fastwave frequency is higher than the slow-wave frequency, so the absolute damping rate of the fast wave, $\zeta_{\mathrm{fa}} \approx 0.03$, is higher than the absolute damping rate of the slow wave, $\zeta_{\mathrm{sl}} \approx 0.02$.

The combined solution, which was found by solving Eq. (89) numerically, was compared to the analytical solution of the envelope approximation of Eq. (89) and to the direct numerical stationary solution of Eqs. (79)-(81), (51), and (57). The numerical, combined, and analytical solutions of these equations are shown in Figs. 98.14 and 98.15 for fast- and slow-wave SBS, respectively. SBS in hydrocarbon plasma was simulated for the OMEGA-like parameters listed above. As in 

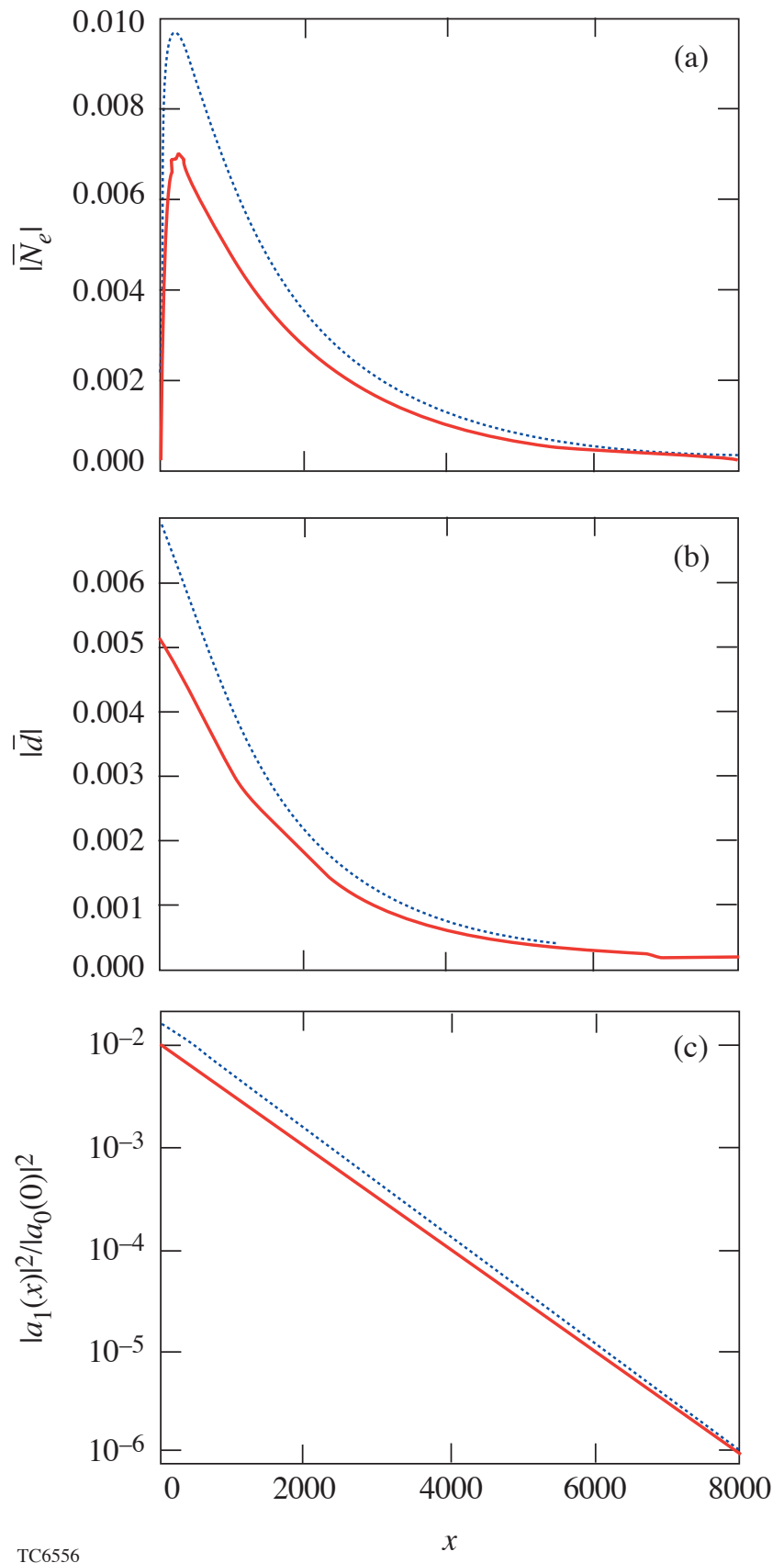

TC6556

$x$

\section{Figure 98.14}

(a) First harmonic of electron density; (b) ponderomotive potential amplitude; (c) Stokes-wave intensity normalized to pump-wave intensity versus distance for steady state of fast-wave SBS. Solutions of linearized equations (51), (57), and (79)-(81) obtained through different methods are compared. Dotted lines represent numerical solution. Dashed lines represent combined solution obtained by numerical solution of Eq. (89). Solid lines represent analytical solution. Numerical and combined solutions are indistinguishable. Simulation parameters are $\lambda_{0}=0.35 \mu \mathrm{m}, I_{L}=2 \times 10^{15} \mathrm{~W} / \mathrm{cm}^{2}$, $T_{e} / T_{l}=T_{e} / T_{h}=4, T_{e}=1 \mathrm{keV}$, and $n_{0 e} / n_{\mathrm{cr}}=0.4$.
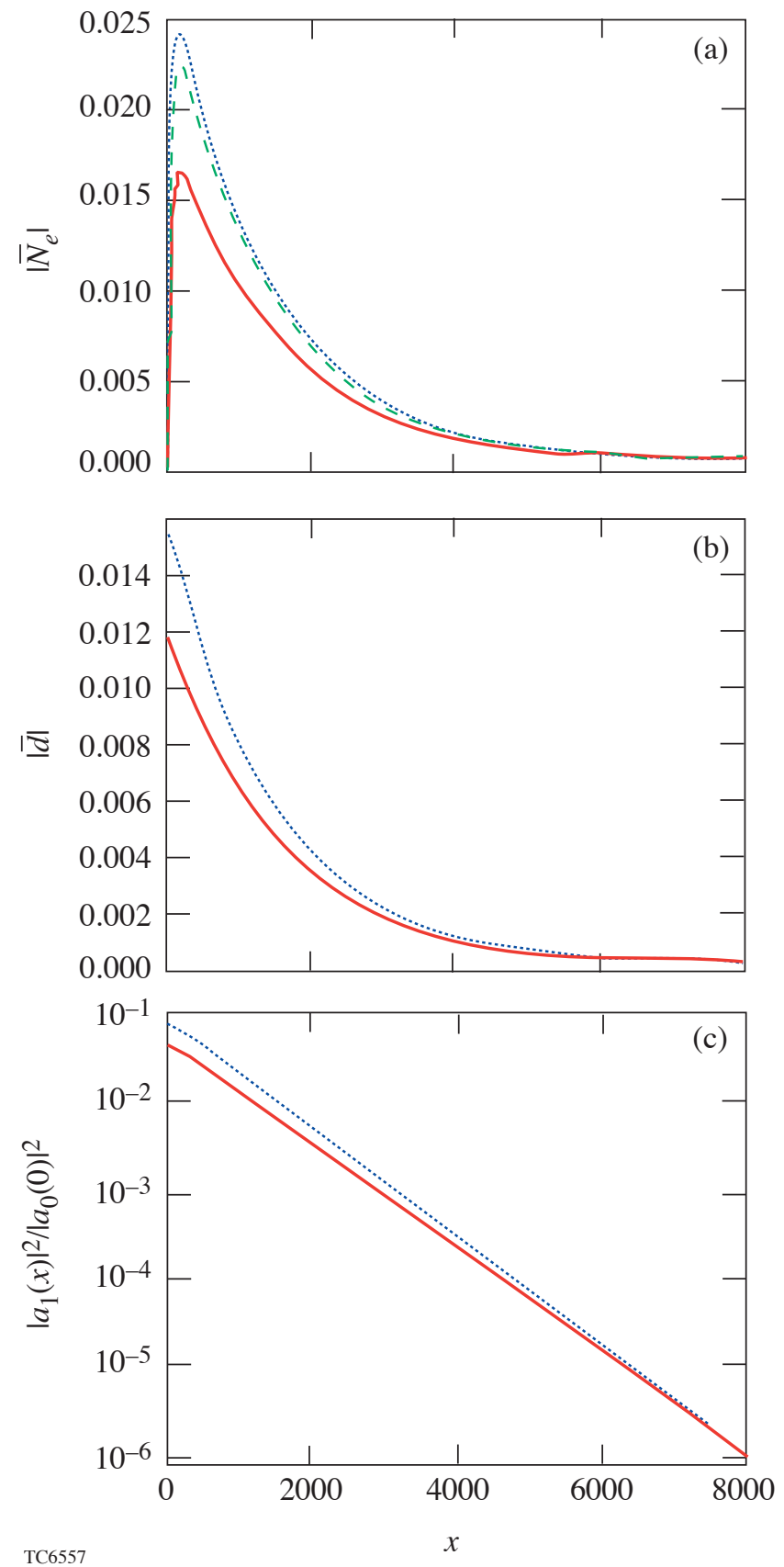

Figure 98.15

(a) First harmonic of electron density; (b) ponderomotive potential amplitude; (c) Stokes-wave intensity normalized to pump-wave intensity versus distance for steady state of slow-wave SBS. Solutions of linearized equations (51), (57), and (79)-(81) obtained through different methods are compared. Dotted lines represent numerical solution. Dashed lines represent combined solution obtained by numerical solution of Eq. (89). Solid lines represent analytical solution. Numerical and combined solutions for ponderomotive potential amplitude and Stokes-wave intensity are indistinguishable. Simulation parameters are the same as in Fig. 98.14. 
the one-ion case, the numerical and combined solutions almost coincide. The fast-wave reflectivity obtained from the numerical solution is $R_{N}=1.89 \%$. The fast-wave reflectivity obtained from the combined solution is $R_{C}=1.88 \%$. The relative difference is $\left(R_{C}-R_{N}\right) / R_{N}=0.2 \%$. Fast-wave reflectivity obtained from the analytical solution is $R_{A}=1.05 \%$. The relative difference is $\left(R_{A}-R_{N}\right) / R_{N}=44 \%$. The slow-wave steady-state reflectivity obtained from the numerical solution is $R_{N}=8.29 \%$. The slow-wave reflectivity obtained from the combined solution is $R_{C}=8.27 \%$. The relative difference is $\left(R_{C}-R_{N}\right) / R_{N}=0.25 \%$. Slow-wave reflectivity obtained from the analytical solution is $R_{A}=4.88 \%$. The relative difference is $\left(R_{A}-R_{N}\right) / R_{N}=41 \%$. For both types of SBS the agreement between the numerical and combined solutions again proves the validity of the computational results. Landaudamping rates in $\mathrm{CH}$ plasmas are high for both fast and slow sound waves $(\zeta / \omega \simeq 0.14)$, which explains why analytical approximations are inaccurate in both cases. For the stated parameters, the Landau damping of the fast wave is stronger than that of the slow wave: $\left(\zeta_{\mathrm{fa}}-\zeta_{\mathrm{sl}}\right) / \zeta_{\mathrm{sl}} \approx 0.5$. The SBS growth rate is inversely proportional to the damping rate. This fact explains why the fast-wave SBS reflectivity is lower than the slow-wave SBS reflectivity: $R_{\mathrm{fa}} / R_{\mathrm{sl}} \approx 0.23$.

The first harmonics of the light- and heavy-ion densities ( $\bar{N}_{l}$ and $\bar{N}_{h}$ ) in fast- and slow-wave SBS are shown in Figs. 98.16 and 98.17. Linear analysis of sound waves in twoion plasmas shows that for fast wave $0<\bar{N}_{h} / \bar{N}_{l}<1$ and for
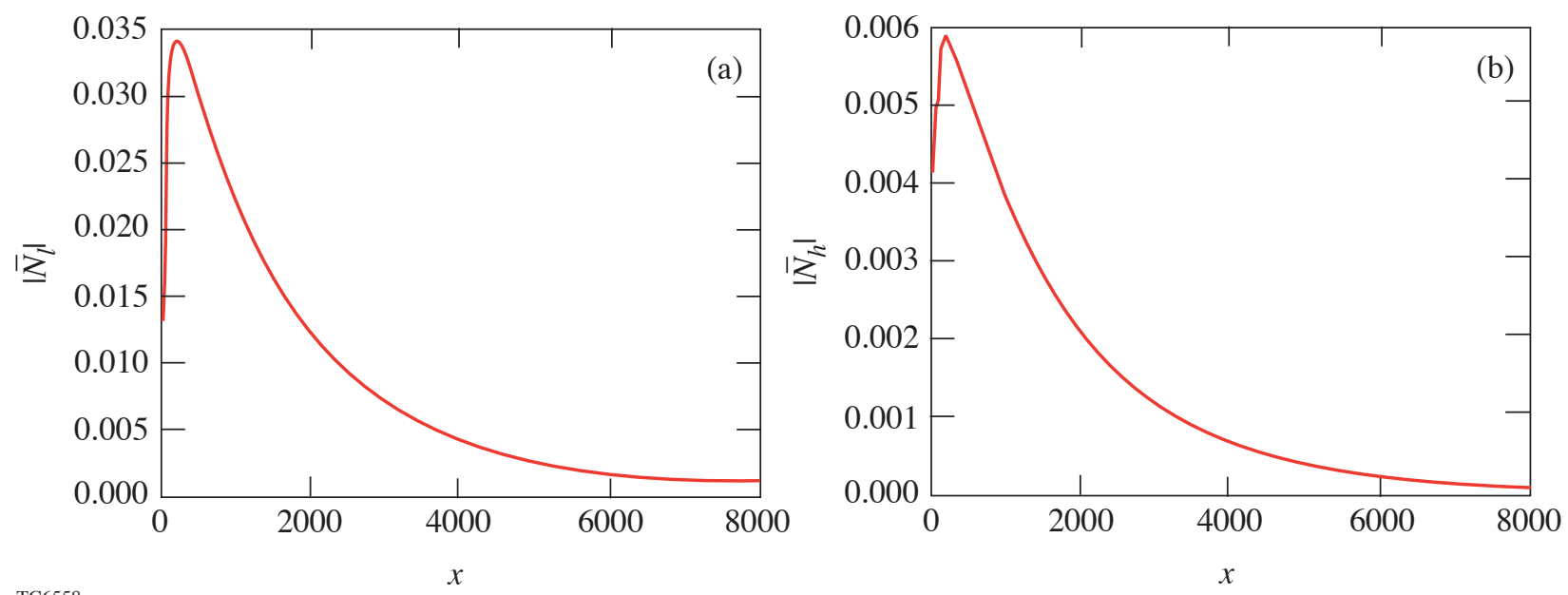

Figure 98.16

(a) First harmonic of light-ion and (b) heavy-ion densities versus distance for steady state of fast-wave SBS. Simulation parameters are the same as in Fig. 98.14.
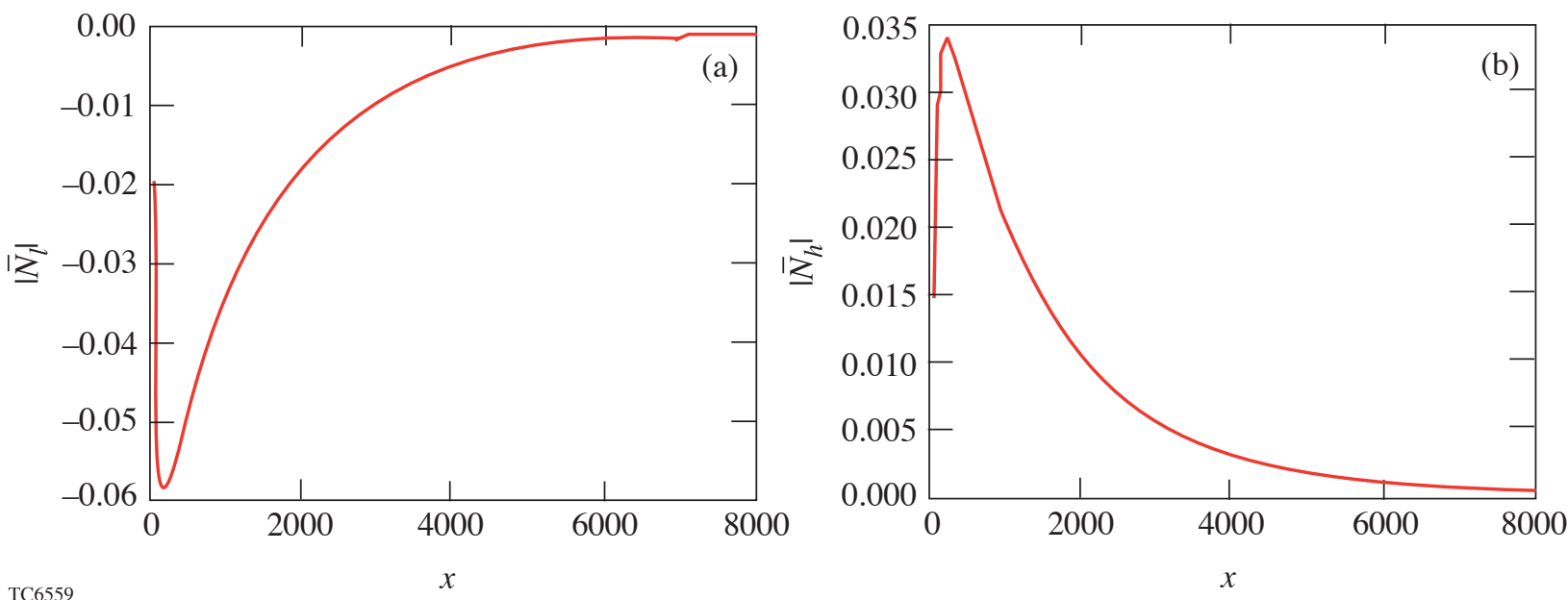

Figure 98.17

(a) First harmonic of light-ion and (b) heavy-ion densities versus distance for steady state of slow-wave SBS. Simulation parameters are the same as in Fig. 98.14 . 
slow wave $\bar{N}_{h} / \bar{N}_{l}<0 .{ }^{17}$ Heavy ions are less mobile than light ions. Their density and velocity perturbations in the highfrequency field of the fast sound wave are smaller than the light-ion perturbations. The negative density ratio is a key feature of the slow sound wave. In the slow wave, light ions shield the electrostatic potential of the heavy ions and, hence, reduce the restoring force. The lower restoring force means lower frequency. At such low frequencies, heavy-ion perturbations are comparable to the light-ion perturbations.

\section{Nonlinear Saturation}

Figures 98.18-98.21 show the effects of hydrodynamic nonlinearities and pump depletion on the saturation of fastand slow-wave SBS. The simulation parameters were listed in Linear Regime of SBS (p. 82). Similar to the one-ion case, the linear solution based on Eqs. (79)-(81), (51), and (57) was compared to the partially nonlinear solution based on Eqs. (79)-(81), (51), (32), and (33) and to the numerically obtained fully nonlinear solution, based on Eqs. (32), (33), and (76)-(78). This allowed us to separate the effects of hydrodynamic nonlinearities and pump depletion.

Steady-state reflectivities of fast-wave SBS are as follows: linear reflectivity $R_{\mathrm{LN}}=1.89 \%$, partially nonlinear reflectivity $R_{\mathrm{PN}}=1.66 \%$, and fully nonlinear reflectivity $R_{\mathrm{FN}}=1.6 \%$. Pump depletion reduces reflectivity by $12 \%$. Ion-acoustic nonlinearities reduce reflectivity by $3.8 \%$.

Steady-state reflectivities of slow-wave SBS are as follows: linear reflectivity $R_{\mathrm{LN}}=8.29 \%$, partially nonlinear reflectivity $R_{\mathrm{PN}}=5.09 \%$, and fully nonlinear reflectivity $R_{\mathrm{FN}}=5.06 \%$. Pump depletion reduces reflectivity by $39 \%$. Ion-acoustic nonlinearities reduce reflectivity by $0.6 \%$.

Nonlinear steepening of fast and slow sound waves was investigated in Ref. 17. In CH plasmas, the fast wave steepens much more than the slow wave. Wave steepening can be considered the generation of higher-order harmonics. Stronger steepening means that the amplitudes of the higher-order harmonics are bigger. These observations explain the difference in the effect of hydrodynamic nonlinearities on the saturation of fast- and slow-wave SBS. The reflectivity of slowwave SBS is bigger than that of the fast-wave SBS. Consequently, the amplitude of the fast sound wave is smaller than that of the slow sound wave. On the other hand, if the fast- and slow-wave amplitudes were comparable, hydrodynamic nonlinearities would affect the fast-wave SBS much more than the slow-wave SBS. If the slow-wave reflectivity is much
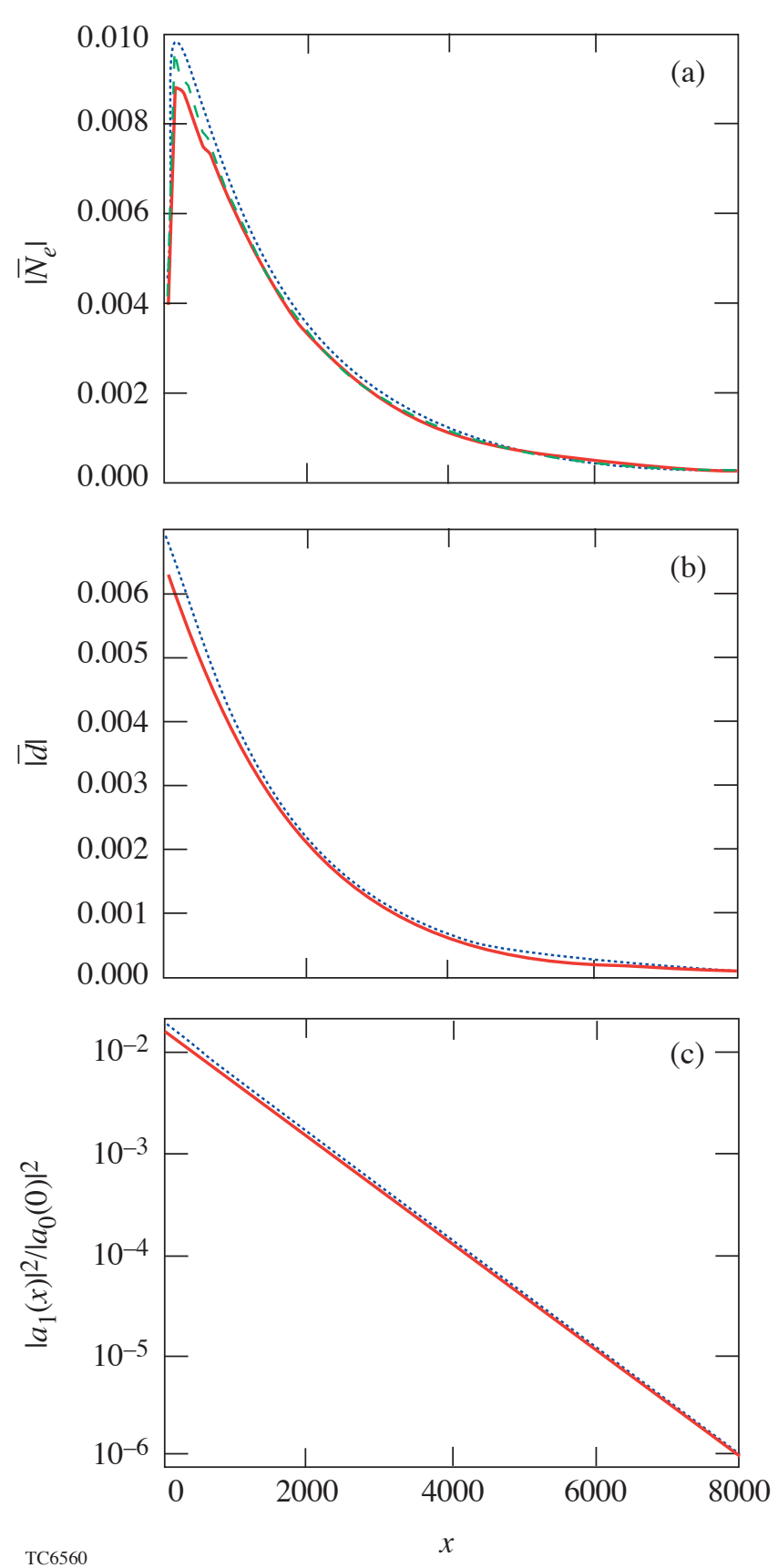

Figure 98.18

(a) First harmonic of electron density; (b) ponderomotive potential amplitude; (c) Stokes-wave intensity normalized to pump-wave intensity versus distance for steady state of fast-wave SBS. Dotted lines represent numerical solution of linearized equations (51), (57), and (79)-(81). Dashed lines represent numerical solution of partially nonlinear equations (32), (33), (51), and (79)-(81). Solid lines represent numerical solution of fully nonlinear equations (32), (33), and (76)-(78). Simulation parameters are the same as in Fig. 98.14. 
higher than the fast-wave reflectivity, the slow-wave amplitude is much bigger than the fast-wave amplitude, in which case the effects of hydrodynamic nonlinearities can be more important for the slow wave than the fast wave. For the stated simulation parameters, the slow-wave reflectivity is not much

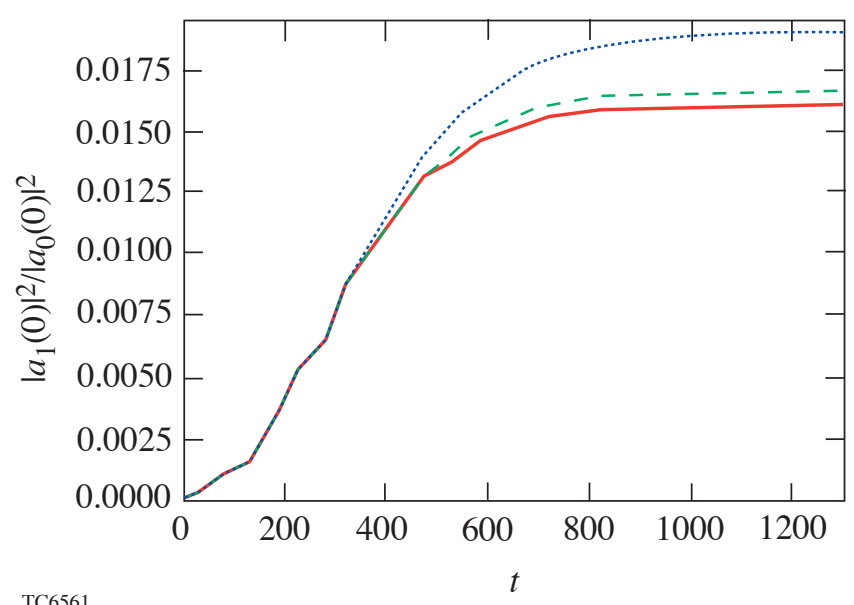

Figure 98.19

Reflectivities versus time for fast-wave SBS. Dotted lines represent numerical solution of linearized equations (51), (57), and (79)-(81). Dashed lines represent numerical solution of partially nonlinear equations (32), (33), (51), and (79)-(81). Solid lines represent numerical solution of fully nonlinear equations (32), (33), and (76)-(78). Simulation parameters are the same as in Fig. 98.14 . higher than the fast-wave reflectivity $\left(R_{\mathrm{fa}} \approx 1 \%, R_{\mathrm{sl}} \approx 5 \%\right)$. In this case, hydrodynamic nonlinearities affect the fast-wave SBS more strongly than slow-wave SBS. Hydrodynamic nonlinearities reduce the fast-wave reflectivity by $3.8 \%$, whereas they only reduce the slow-wave reflectivity by $0.6 \%$

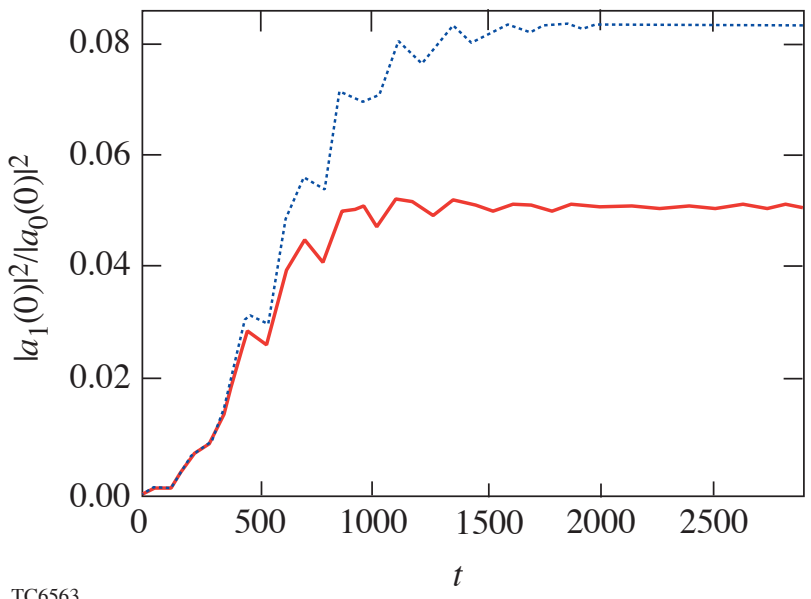

Figure 98.21

Reflectivities versus time for slow-wave SBS. Dotted lines represent numerical solution of linearized equations (51), (57), and (79)-(81). Dashed lines represent numerical solution of partially nonlinear equations (32), (33), (51), and (79)-(81). Solid lines represent numerical solution of fully nonlinear equations (32), (33), and (76)-(78). Simulation parameters are the same as in Fig. 98.14 .
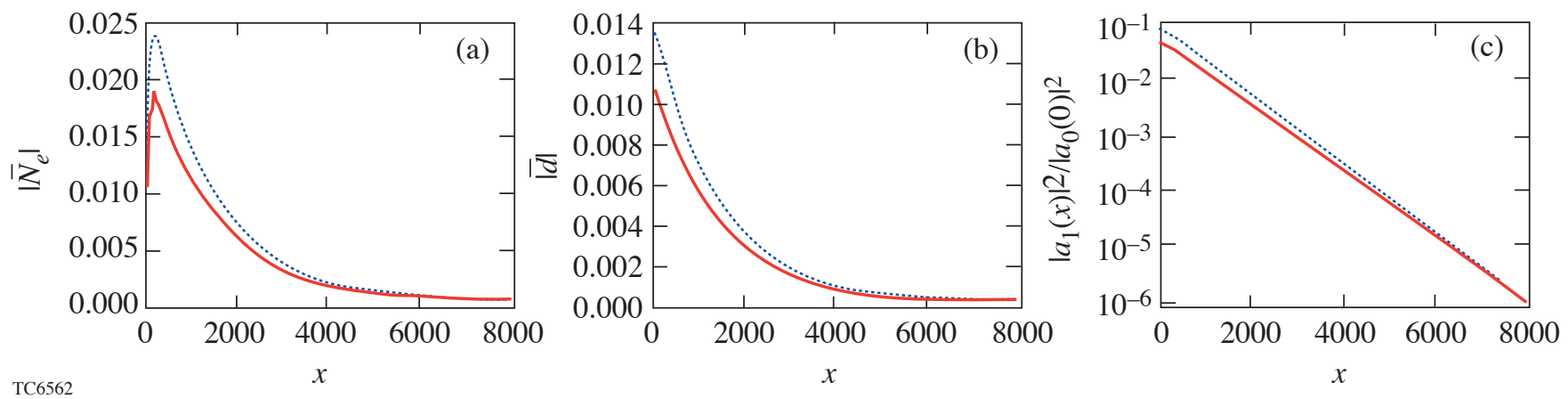

Figure 98.20

(a) First harmonic of electron density; (b) ponderomotive potential amplitude; (c) Stokes-wave intensity normalized to pump-wave intensity versus distance for steady state of slow-wave SBS. Dotted lines represent numerical solution of linearized equations (51), (57), and (79)-(81). Dashed lines represent numerical solution of partially nonlinear equations (32), (33), (51), and (79)-(81). Solid lines represent numerical solution of fully nonlinear equations (32), (33), and (76)-(78). Simulation parameters are the same as in Fig. 98.14. 
The pump-wave intensities in fast- and slow-wave SBS are plotted as functions of distance in Figs. 98.22 and 98.23, respectively. Pump intensities are normalized to input pump intensity. The output pump intensity

$$
\left|A_{0}(L)\right|^{2} / I=1-R_{\mathrm{PN}}+\left|A_{1}(L)\right|^{2} / I
$$

Light scattering by the slow sound wave decreases the pump intensity much more than scattering by the fast sound wave because the reflectivity of slow-wave SBS is higher than that of fast-wave SBS.

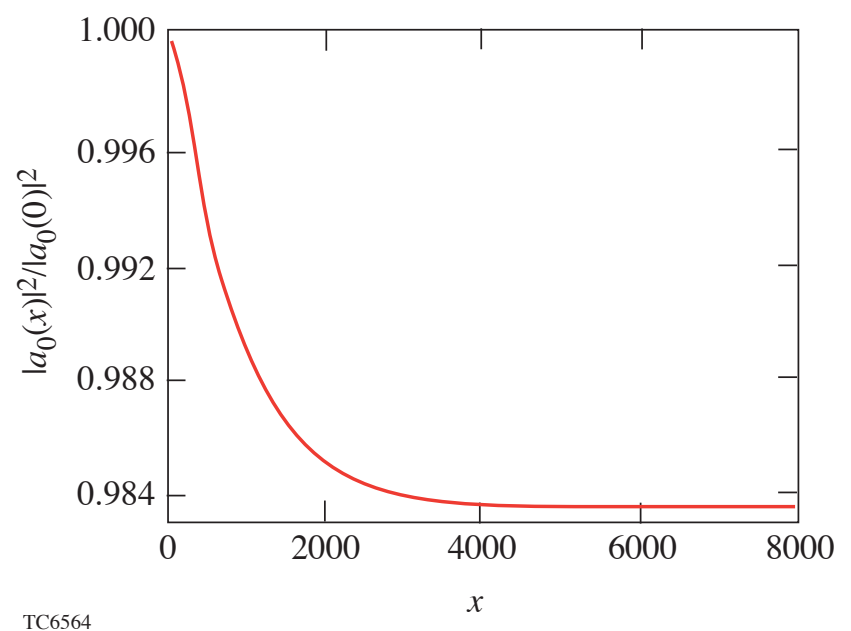

Figure 98.22

Pump-wave intensity normalized to input pump intensity versus distance for steady state of fast-wave SBS. Simulation parameters are the same as in Fig. 98.14.

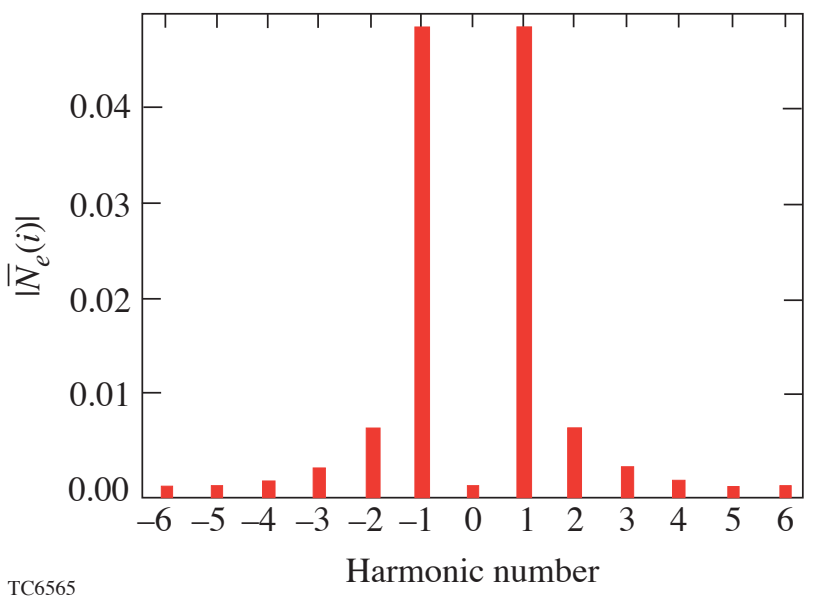

Figure 98.23

Fourier spectra of electron density near the point where it has maximal amplitude for steady state of fast-wave SBS. Simulation parameters are the same as in Fig. 98.14.
Higher-order harmonic generation is demonstrated by plotting discrete Fourier spectra of electron density near the point where it has maximal amplitude. The absolute values of the steady-state harmonics associated with the fast- and slowwave SBS are shown in Figs. 98.24 and 98.25, respectively. The second- and higher-order harmonics are small in both the fast and slow sound waves because the amplitudes of both sound waves are small. The higher-order harmonics of fast-

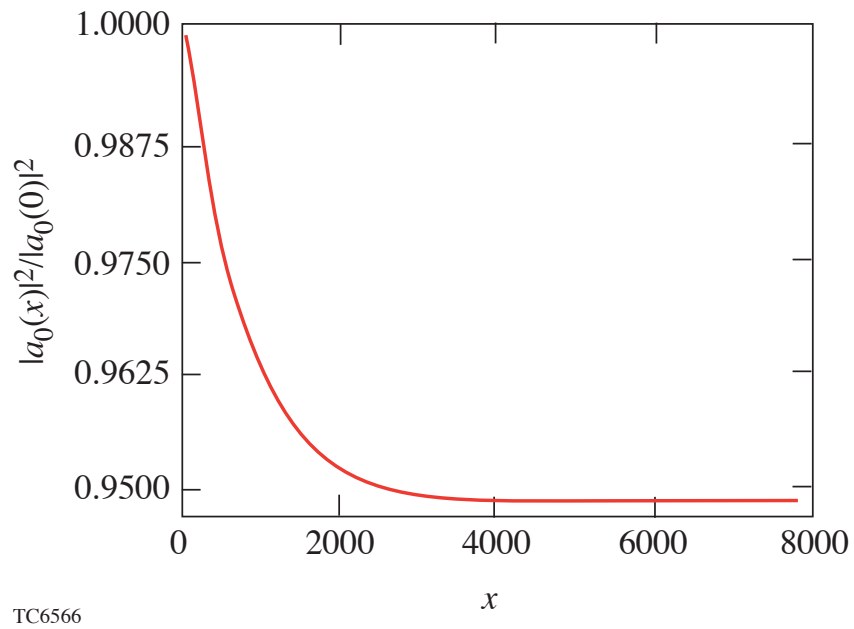

Figure 98.24

Pump-wave intensity normalized to input pump intensity versus distance for steady state of slow-wave SBS. Simulation parameters are the same as in Fig. 98.14.

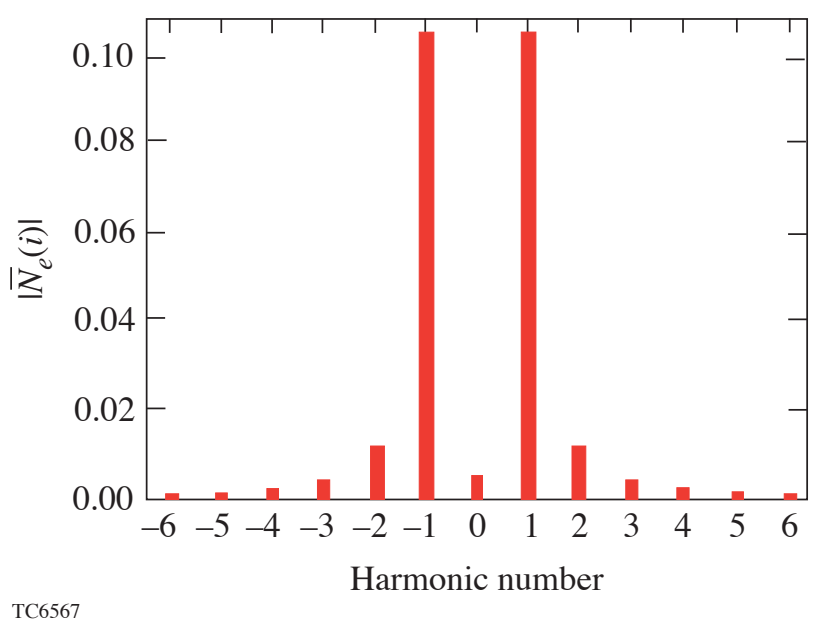

Figure 98.25

Fourier spectra of electron density near the point where it has maximal amplitude for steady state of slow-wave SBS. Simulation parameters are the same as in Fig. 98.14. 
wave SBS are larger than the higher-order harmonics of slowwave SBS because in $\mathrm{CH}$ plasmas the fast wave steepens much more than the slow wave. Consequently the effects of hydrodynamic nonlinearities on both the fast- and slow-wave SBS are small. They are noticeable for the fast-wave SBS but negligible for the slow-wave SBS.

\section{Summary}

A fluid model with phenomenological damping terms was used to study convective SBS in one- and two-ion plasmas. The Landau-damping rates were evaluated using formulas from kinetic theory. A fluid code was developed and tested by comparing its predictions to analytical formulas for SBS in the linear regime. SBS was simulated in carbon and hydrocarbon $(\mathrm{CH})$ plasmas with OMEGA-like parameters. Two types of sound waves (fast and slow) exist in two-ion plasmas, each of which can participate in SBS. SBS from fast and slow sound waves were separated by choosing the beat frequency of the ponderomotive potential. The fast-wave reflectivity is lower than the slow-wave reflectivity because the Landau damping of the fast wave is stronger than the Landau damping of the slow wave. Effects of hydrodynamic nonlinearities and pump depletion on saturation of SBS in one- and two-ion plasmas were compared. The pump depletion significantly reduces reflectivity in one- and two-ion plasmas. The hydrodynamic nonlinearities are important for the SBS in one-ion carbon plasmas and noticeable for the fast-wave SBS in two-ion $\mathrm{CH}$ plasmas, but negligible for the slow-wave SBS.

\section{Acknowledgment}

The authors would like to thank D. D. Meyerhofer, W. Seka, J. Myatt, V. N. Goncharov, K. Vagin, I. Kuzora, and S. Urupin for fruitful discussions on this topic. This work was supported by the Department of Energy (DOE) Office of Inertial Confinement Fusion, under Cooperative Agreement No. DE-FC03-92SF19460, the University of Rochester, the New York State Research and Development Authority, and the Civilian Research and Development Foundation, under Award No. RP1-2268. The support of DOE does not constitute an endorsement by DOE of the views expressed in this article.

\section{REFERENCES}

1. W. L. Kruer, The Physics of Laser-Plasma Interactions, Frontiers in Physics, Vol. 73, edited by D. Pines (Addison-Wesley, Redwood City, CA, 1988), Chap. 4, pp. 37-43.

2. R. L. McCrory and J. M. Soures, in Laser-Induced Plasmas and Applications, edited by L. J. Radziemski and D. A. Cremers (Marcel Dekker, New York, 1989), pp. 207-268.

3. N. A. Krall and A. W. Trivelpiece, Principles of Plasma Physics (San Francisco Press, Inc., San Francisco, 1986).
4. J. A. Heikkinen, S. J. Karttunen, and R. R. E. Salomaa, Phys. Fluids 27, 707 (1984).

5. W. Rozmus et al., Phys. Fluids B 4, 576 (1992).

6. B. I. Cohen et al., Phys. Plasmas 4, 956 (1997).

7. B. D. Fried, R. B. White, and T. K. Samec, Phys. Fluids 14, 2388 (1971).

8. H. X. Vu, J. M. Wallace, and B. Bezzerides, Phys. Plasmas 1, 3542 (1994).

9. E. A. Williams, R. L. Berger, R. P. Drake, A. M. Rubenchik, B. S. Bauer, D. D. Meyerhofer, A. C. Gaeris, and T. W. Johnston, Phys. Plasmas 2, 129 (1995).

10. M. V. Kozlov and C. J. McKinstrie, Laboratory for Laser Energetics Report No. 321, NTIS document No. DOE/SF/19460-385, University of Rochester (2001). Copies may be obtained from the National Technical Information Service, Springfield, VA 22161.

11. J. D. Hoffman, Numerical Methods for Engineers and Scientists (McGraw-Hill, New York, 1992).

12. W. H. Press et al., Numerical Recipes in FORTRAN: The Art of Scientific Computing, 2nd ed. (Cambridge University Press, Cambridge, England, 1992).

13. N. M. Kroll, J. Appl. Phys. 36, 34 (1965); A. V. Maximov et al., Phys. Plasmas 7, 4227 (2000).

14. C. J. McKinstrie, R. E. Giacone, and E. A. Startsev, Phys. Plasmas 6, 463 (1999).

15. V. V. Kurin and G. V. Permitin, Sov. J. Plasma Phys. 8, 365 (1982).

16. S. J. Karttunen and R. R. E. Salomaa, Phys. Lett. A 72, 336 (1979).

17. M. V. Kozlov and C. J. McKinstrie, Phys. Plasmas 9, 3783 (2002). 


\section{Imprint Efficiency Measurements in Laser-Driven Plastic Foils Using Beams with Different Angles of Incidence}

\section{Introduction}

The goal of the direct-drive approach to inertial confinement fusion (ICF) ${ }^{1,2}$ is to uniformly implode a spherical target with deuterium-tritium (DT) fuel using a large number of overlapped laser beams. A combination of high temperature and high areal density $(\rho R)$ in the DT fuel at peak compression is necessary to ignite the target and achieve high gain. ${ }^{1-3}$ The most-significant factor that limits the implosion performance is the unstable growth of target perturbations. As a result of this growth, the fuel temperature and compression may be reduced, leading to a reduction in the thermonuclear yield. The target perturbations in direct-drive ICF include existing imperfections of the inner and outer target surfaces and are dominated by modulations seeded (or imprinted) by the spatial laser nonuniformities; ${ }^{4-29}$ therefore, understanding and controlling laser imprinting are crucial to the success of direct-drive ICF.

Spatial modulations in laser intensity are imprinted into the target in the first few hundred picoseconds of the drive. As the laser light is applied to the target, the pressure created by the target ablation launches a shock wave that compresses the target. ${ }^{30,31}$ Any nonuniformities in the laser drive modulate the ablation pressure. The modulations in surface acceleration provide the seeds for hydrodynamic instabilities. Later, as a large volume of plasma develops, the laser modulations decouple from the target surface, smoothing the ablation pressure. The imprinted front-surface (or ablation-surface) perturbations continue to evolve as the shock-driven RichtmyerMeshkov (RM) instability causes the modulations to grow; the ablation stabilizes this growth. ${ }^{18,32}$ As a result, the ablationsurface nonuniformities oscillate during the shock propagation to the rear surface of the target. The amplitude and frequency of these oscillations are defined by the modulation wavelength, the sound speed, the ablation, and the expanding (or "blowoff") plasma velocities. ${ }^{32}$ Because the shock is launched by a modulated laser drive, the shock front is also distorted. The amplitude of this distortion oscillates as it propagates through the target with a frequency determined by the modulation wavelength and the drive intensity. ${ }^{30,31}$ When the shock front reaches the rear surface of the target, it sends the rarefaction wave back to the ablation surface; shortly thereafter, the target starts to accelerate. During the acceleration phase, the ablation-surface modulations grow exponentially due to Rayleigh-Taylor (RT) instability. ${ }^{2,3}$

A number of techniques have been developed to reduce laser imprinting in direct-drive ICF facilities. A combination of distributed phase plates (DPP's), ${ }^{33}$ polarization smoothing (PS), ${ }^{34}$ and smoothing by spectral dispersion (SSD) ${ }^{35}$ is employed on the OMEGA laser. ${ }^{36}$ Induced spatial incoherence $(\text { ISI })^{37}$ is used on the NIKE laser system. Partially coherent light (PCL) ${ }^{38}$ in combination with random-phase plates is used on the GEKKO-XII laser facility. Targets with foam-buffered layers, high- $Z$ overcoat, and a combination of the two have been demonstrated to reduce imprinting. ${ }^{5-7,11,16,20,23-25,27-29}$

The first measurements ${ }^{5}$ of laser-imprinted modulations were performed using a side-on geometry, where the diagnostic $\mathrm{x}$ rays penetrate a planar target in the direction perpendicular to its motion. Almost all subsequent imprinting studies were performed using a face-on geometry where the diagnostic $\mathrm{x}$ rays penetrate the target in the direction along its motion, allowing more-quantitative measurements of target perturbations. Face-on radiography is sensitive to variations in the target density thickness, or areal density $(\rho R)$, which includes not only the ablation-front modulations (existing or laser imprinted), $\delta\left[\rho R_{\mathrm{abl}}(t)\right]$ at time $t$, but also any shock-front modulation in the bulk of the target, $\delta\left[\rho R_{\mathrm{sh}}(t)\right]$ :

$$
\delta[\rho R(t)]=\delta\left[\rho R_{\mathrm{abl}}(t)\right]+\delta\left[\rho R_{\mathrm{sh}}(t)\right]
$$

Early imprint experiments ${ }^{8-10,12,14,15,17-19}$ were performed at or before shock breakout on the rear surface of the target (before the onset of the RT growth), when the ablation- and shock-front modulations are of the same order of magnitude, $\delta\left[\rho R_{\mathrm{abl}}(t)\right] \cong \delta\left[\rho R_{\mathrm{sh}}(t)\right]$. Intended to be measurements of the ablation-surface imprinted perturbations (initial seed for the RT instability), the resulting areal-density modulations also included the shock-front perturbations. ${ }^{30,31}$ Later experiments 
observed shock-front and ablation-surface evolutions during shock transit before the beginning of the RT growth. ${ }^{30,31,39-41}$

To quantify imprinted modulation levels, imprint efficiency measurements ${ }^{10,14,26}$ have used the acceleration-phase RT growth to magnify the ablation-front modulations in order to separate them from the shock-front modulations. Figure 98.26 (from Ref. 34) schematically presents the idea behind these experiments using simulations by the 2-D code ORCHID. ${ }^{42}$ The solid curve shows the evolution of the areal-density modulation of an initially smooth target driven by a laser having a single-mode intensity modulation at $60-\mu \mathrm{m}$ spatial wavelength, while the dotted curve shows the evolution of the single-mode, 60- $\mu$ m-wavelength, preimposed perturbation driven by a spatially perfect laser. The solid curve starts at zero and rises as imprinting begins, while the dotted curve starts at its preimposed level. The RT growth (starting at 400 ps) amplifies the imprinted and imposed ablation-front perturbations in the same manner and, when ablation-front modulations become higher than shock-front modulations, $\delta\left[\rho R_{\mathrm{abl}}(t)\right]>\delta\left[\rho R_{\mathrm{sh}}(t)\right]$, the areal-density evolutions become similar (dotted and solid curves are parallel after $0.5 \mathrm{~ns}$ ). The

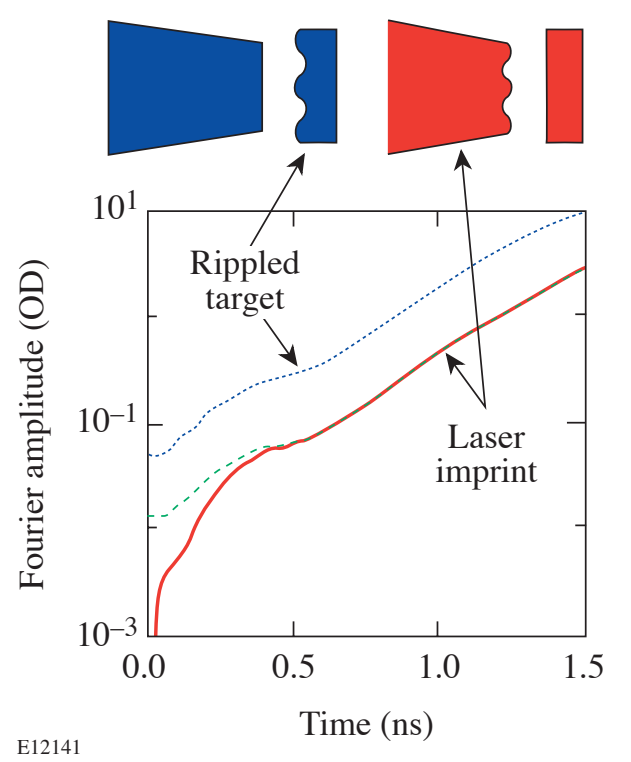

Figure 98.26

Definition of the equivalent surface amplitude using an ORCHID simulation of a single-mode, $60-\mu \mathrm{m}$-wavelength, imprinted modulation (solid curve) calibrated to a preimposed (dotted curve) modulation (from Ref. 34). The equivalent surface amplitude of imprinting at a particular mode number $k$ is defined by extrapolating (dashed curve) the temporal evolution of the imprinted modulation (solid curve) back to $t=0$, using the behavior of the preimposed mode (dotted curve) and Eq. (2). equivalent surface amplitude of imprinting at a particular mode number $k$ is defined by extrapolating (dashed curve) the temporal evolution of the imprinted modulation (solid curve) back to $t=0$ using the behavior of the preimposed mode (dotted curve):

$$
\begin{aligned}
\eta_{\text {imp }}(k, t=0)= & \eta_{\text {pre }}(k, t=0) \\
& \times\left\{\delta\left[\rho R_{\text {imp }}(k, t)\right] / \delta\left[\rho R_{\text {pre }}(k, t)\right]\right\},
\end{aligned}
$$

where $\eta_{\text {pre }}(k, t=0)$ is the initial amplitude of the preimposed modulation and $\delta\left[\rho R_{\text {imp }}(k, t)\right]$ and $\delta\left[\rho R_{\text {pre }}(k, t)\right]$ are the measured areal-density modulations of imprinted and imposed perturbations during linear RT growth, respectively. This technique is valid when (1) the amplitudes of imprinted and preimposed modulations are in the linear regime of the RT growth, and (2) the measurements are taken when the ablation-front modulations are large enough to dominate the measurements. The imprint efficiency $E(k)$ was defined ${ }^{10,14}$ as the initial amplitude of equivalent surface modulation $\eta_{\text {imp }}(k, t=0)$, normalized to the relative laser modulation $\delta I(k) / I$, that produced it:

$$
E(k)=\eta_{\text {imp }}(k, t=0) /[\delta I(k) / I] .
$$

In direct-drive implosions, a spherical shell is illuminated by a large number of overlapping laser beams. Each beam diameter is roughly equal to that of the target; therefore, different parts of the beam irradiate the target at different angles of incidence: the central part of the beam is nearly normally incident to the target, while the outer parts of the beam irradiate the target at oblique angles. As a result it is important to investigate the effect of the beam angle of incidence on imprint efficiency. Recently, the imprint efficiency measurements for three different angles of incidence were performed for the first time in targets and laser intensities relevant to the spherical implosion program on OMEGA. ${ }^{43}$ This article presents details of the techniques and analysis of the imprint efficiency measurements and is considered complimentary to Ref. 43.

\section{Experimental Configuration}

Figure 98.27(a) shows schematically the experimental configuration, previously used in a number of experiments. ${ }^{20-22,26,34}$ The 20 - $\mu$ m-thick plastic targets were irradiated by 351-nm laser light at $\sim 2 \times 10^{14} \mathrm{~W} / \mathrm{cm}^{2}$ using seven overlapped beams with a 3 -ns square pulse shape on the 
OMEGA laser system. ${ }^{36}$ The imprint efficiencies at $60-\mu \mathrm{m}$ spatial wavelength were with and without $0.2-\mathrm{THz}$ SSD. ${ }^{35}$ Targets with preimposed, single-mode, two-dimensional, $60-\mu \mathrm{m}$ sinusoidal perturbations were used to determine the imprint efficiency: one target with $0.125-\mu \mathrm{m}$ initial amplitude and the other with $0.05-\mu \mathrm{m}$ initial amplitude. The temporal growth of target perturbations was measured using x-ray, faceon radiography. The targets were backlit with $\mathrm{x}$ rays produced by a uranium backlighter located $9 \mathrm{~mm}$ away from the driven foil and irradiated at $\sim 1 \times 10^{14} \mathrm{~W} / \mathrm{cm}^{2}$ using 12 additional beams. X rays transmitted through the target and a $3-\mu$ m-thick aluminum debris shield (located between the backlighter and the driven foil) were imaged by the $8-\mu \mathrm{m}$ pinhole array on a framing camera filtered with $6 \mu \mathrm{m}$ of aluminum. ${ }^{20-22}$ This yielded the highest sensitivity for the average photon energy of $\sim 1.3 \mathrm{keV}$. The distance between the target and the pinhole array was $2.5 \mathrm{~cm}$, and the magnification was 14.4. The framing camera recorded eight images in each shot with a temporal resolution of $\sim 80 \mathrm{ps}$ and a spatial resolution in a target plane of $\sim 10 \mu \mathrm{m} .{ }^{44}$ The framing camera images were captured on Kodak T-Max 3200 film, which was digitized with a 20- $\mu$ m-sq scanning aperture. The measured target optical depth (which is proportional to the target areal density) is the natural logarithm of the intensity-converted images of a target.

Figure 98.27(b) shows the laser-beam configuration. Six beams (numbers 34, 36, 38, 41, 43, and 49) were incident at $23^{\circ}$ to a target normal, while one beam (number 48) was incident at $48^{\circ}$ to a target normal. The $23^{\circ}$ beams had DPP's ${ }^{33}$ and PS. ${ }^{34}$ An equivalent-target-plane image of one such beam is shown in Fig. 98.28(a). These beams had a broadband spectrum of modulations with the smallest features having spatial wavelengths of $\sim 2.5 \mu \mathrm{m}$. Beam 48 [shown in Fig. 98.28(b)], incident at a more-oblique $48^{\circ}$ angle, had 2-D, 60- $\mu$ m-wavelength intensity modulations (together with several higher harmonics) to distinguish it from the $23^{\circ}$ beams. The beam modulations were oriented along the direction of its propagation, so the imprinted target modulations had the same wavelengths as the laser modulations. The 2-D laser modulations were used to
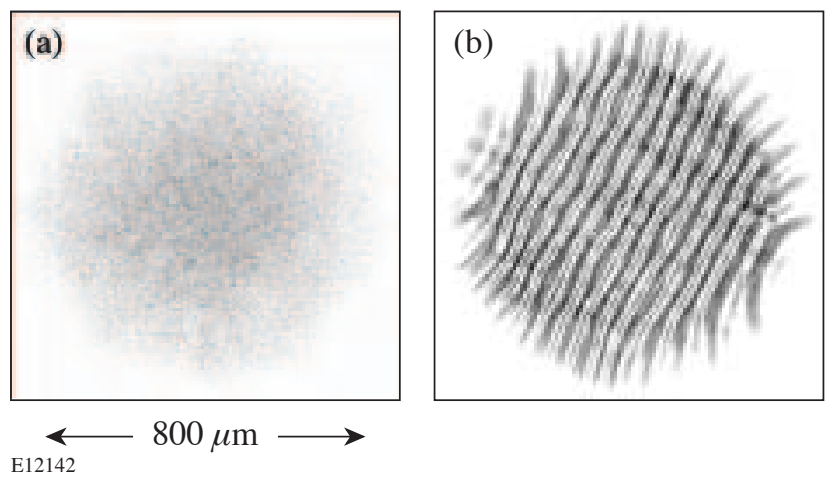

Figure 98.28

Beam images of the (a) $23^{\circ}$ beam having 3-D broadband perturbations and (b) $48^{\circ}$ beam having $2-\mathrm{D}, 60-\mu \mathrm{m}$-wavelength perturbation. (a)
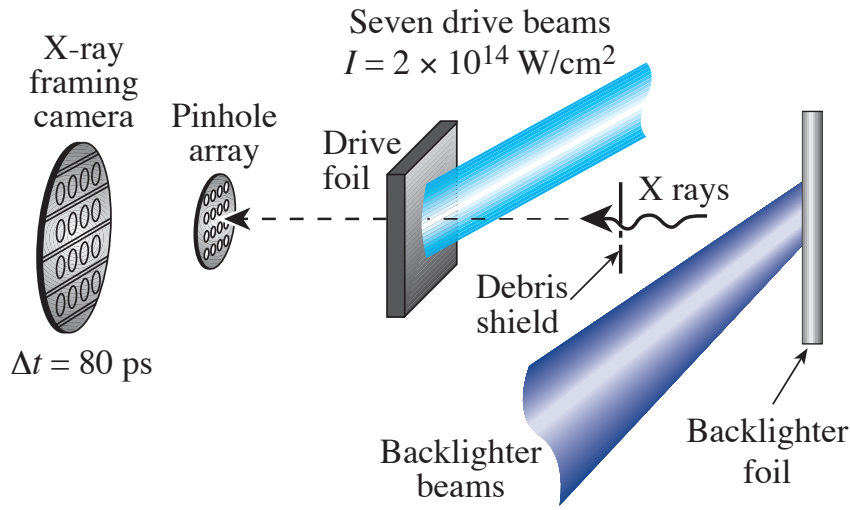

E12148 (b)

$\mathrm{CH}$ foil with horizontal $60-\mu \mathrm{m}-$ wavelength perturbation

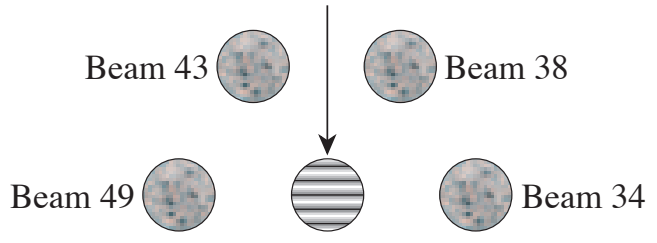

Beam $41 \%$ Beam 36

Beam 480

\section{Figure 98.27}

(a) Experimental configuration (from Ref. 22); (b) beam and target configuration. Diagonal 2-D perturbations at $60-\mu \mathrm{m}$ wavelength come from beam 48 incident at $48^{\circ}$ to a target normal, while 3 -D broadband perturbations come primarily from beams $34,36,38,41$, 43 , and 49 incident at $23^{\circ}$ to the target normal. The target has a horizontal, preimposed, single-mode, $60-\mu \mathrm{m}$-wavelength perturbation used for calibration. 
separate perturbations caused by the $48^{\circ}$ beam from the 3-D broadband modulations caused by the $23^{\circ}$ beams in the radiogram of the driven target. Figure 98.29(a) shows the profile of the relative intensity incident on the target (averaged in the direction along the 2-D perturbations), calculated using measured beam intensities [Figs. 98.28(a) and 98.28(b)] and taking into account experimentally measured beam energies, effects of beam overlap, and obliquity angles. Figure 98.29(b) presents the Fourier amplitude of this lineout showing the laser perturbations at $60-\mu \mathrm{m}$ wavelength (together with the higher harmonics) clearly distinguishable from the other broadband laser modulations. The laser modulations were analyzed in nine different square areas (with a box size of $L=300 \mu \mathrm{m}$, the same size as in the target $\mathrm{x}$-ray radiographs shown later) of $\sim 800-\mu \mathrm{m}$ laser spots, and it was found that the modulations were reasonably constant across the laser-spot size. The amplitudes of relative laser modulations at a spatial frequency of $17 \mathrm{~mm}^{-1}$ (corresponding to a spatial wavelength of $60 \mu \mathrm{m}$ ) were $6.3 \pm 0.4 \%$ for the two-dimensional modulation (from the $48^{\circ}$ beam) and $0.54 \pm 0.09 \%$ for the broadband modulations (from the $23^{\circ}$ beams), as calculated in the nine different areas.
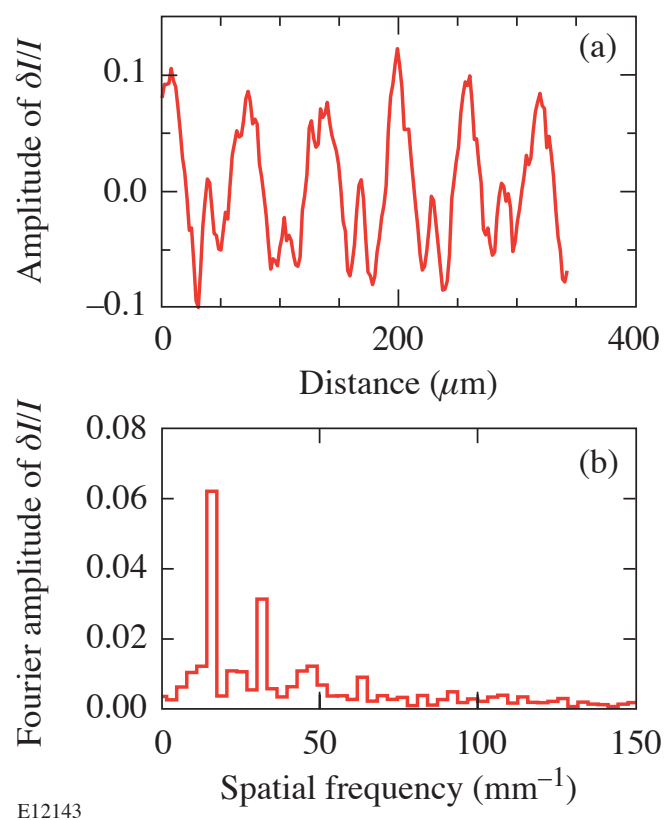

Figure 98.29

(a) Profile of the relative intensity incident on the target averaged in the direction along the 2-D laser perturbations. (b) Fourier amplitude of this profile showing the laser perturbations at a spatial frequency of $17 \mathrm{~mm}^{-1}$ (corresponding to the $60-\mu \mathrm{m}$ wavelength) together with the higher harmonics clearly distinguishable from the other broadband laser modulations.

LLE Review, Volume 98

\section{Experimental Results}

Figure 98.30(a) presents one of the eight images of target optical-depth modulations taken at $\sim 1.9 \mathrm{~ns}$ after the beginning of the laser drive for the shot without SSD. The corresponding Fourier-space image of the target optical-depth modulations is shown in Fig. 98.30(b). Two-dimensional perturbations, imprinted from the $48^{\circ}$ beam, are diagonal across the real-space image; they have distinctive first- and second-harmonic peaks in the Fourier-space image. The preimposed, 60- $\mu \mathrm{m}$-wavelength perturbation is horizontal in the real-space image; therefore it has two vertical peaks in the Fourier-space image. The 3-D features in the real-space image are imprinted from the broadband perturbations of $23^{\circ}$ beams; these perturbations are located in the broad area of the Fourier-space image. The profiles of the 2-D imprinted and preimposed modulations are presented in the Fig. 98.31. Figure 98.31(a) shows the opticaldepth profile of the 2-D imprinted modulation averaged along the modulations, while Fig. 98.31(b) shows the profile of the 2-D preimposed modulation, averaged along the horizontal direction. The higher optical depth corresponds to thinner areas of the targets, or bubbles, while the lower optical depth corresponds to thicker target areas, or spikes. The profile of the imprinted optical-depth modulation resembles the profiles of the laser modulation [compare Figs. 98.29(a) and 98.31(a)]. (a)

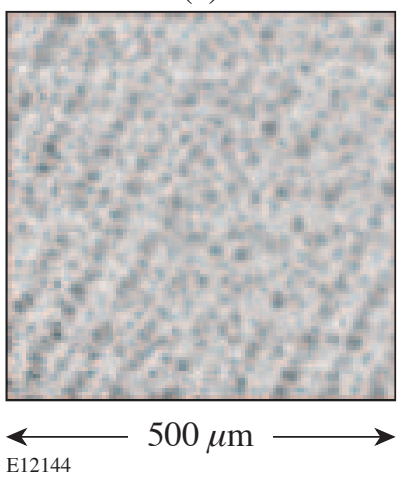

(b)

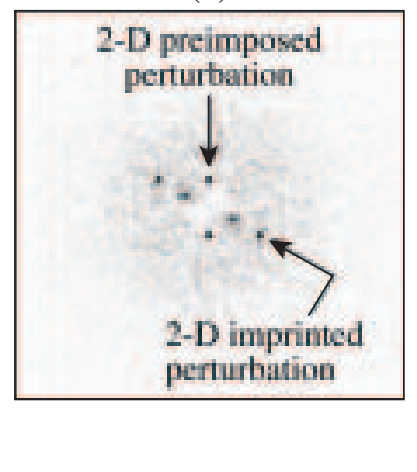

Figure 98.30

(a) Image of the target optical-depth modulations taken at $\sim 1.9$ ns after the beginning of the laser drive for the shot without SSD. (b) Fourier-space image of the target optical-depth modulations showing peaks for a diagonal 2-D, 60- $\mu \mathrm{m}$ wavelength and its second-harmonic imprinted modulations, and 2-D, $60-\mu \mathrm{m}$-wavelength, preimposed modulations in the vertical direction.

Figure 98.32 summarizes the results of the measured growth for all $60-\mu \mathrm{m}$-wavelength perturbations with imprinted modulations shown in Fig. 98.32(a) and preimposed modulations in Fig. 98.32(b) for two shots with and without SSD. Twodimensional imprinted modulations from the $48^{\circ}$ beam are 
shown by the upper data (diamonds) in Fig. 98.32(a), and 3-D broadband modulations from the $23^{\circ}$ beams are shown by the lower data (squares). The amplitudes of imprinted modulations are separated by about $0.5 \mathrm{~ns}$ in shots with and without SSD [Fig. 98.32(a)]. This is because the SSD reduces the initial amplitudes of imprinted modulations by a factor of $\sim 2.5$, and it takes longer for the RT growth to bring them to the same levels as for the shot without SSD. The initial amplitudes of preimposed modulations were $0.125 \mu \mathrm{m}$ and $0.05 \mu \mathrm{m}$ for the shots without and with SSD, respectively.

To satisfy the conditions for imprint efficiency measurements described in the Introduction, the growth measurements of $60-\mu \mathrm{m}$-wavelength perturbations were performed in the linear regime of RT instability. To confirm that the measured modulations were below the RT saturation levels, ablation-front amplitudes $\eta(k, t)$ were estimated from the measured optical-depth modulations $\operatorname{OD}(k, t)$, the measured undriven target attenuation length $\lambda_{\text {eff }}=10 \mu \mathrm{m}$, and the calculated (1-D hydrocode LILAC) ${ }^{45}$ target compression
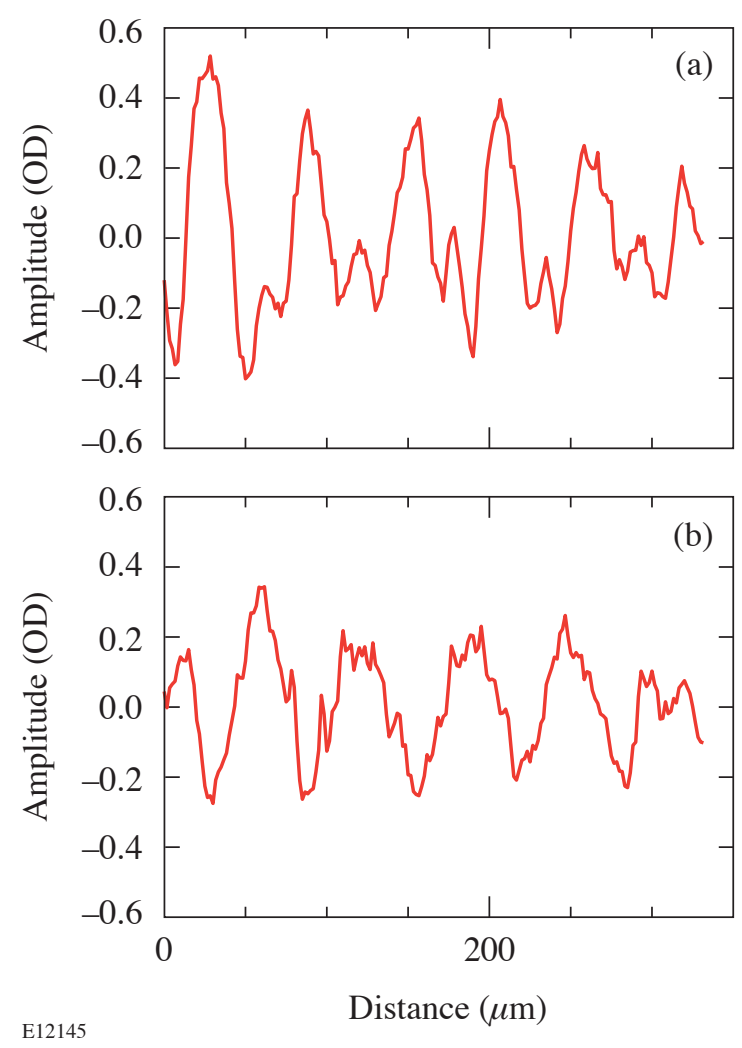

Figure 98.31

Optical-depth profiles of (a) the 2-D imprinted modulation and (b) the 2-D preimposed modulation taken at $\sim 1.9 \mathrm{~ns}$ after the beginning of the laser drive.
$C_{p} \sim 3, \eta(k, t)=\mathrm{OD}(k, t) \times \lambda_{\text {eff }} / C_{p}$. The amplitudes of 2-D, single-mode perturbations were calculated to be below $1 \mu \mathrm{m}$ at all times for both shots (the 2-D saturation amplitude for the $\lambda=60-\mu \mathrm{m}$ wavelength is $S_{2-\mathrm{D}}=6 \mu \mathrm{m}$ ), and the amplitudes of 3 -D, broadband perturbations were calculated to be below $0.1 \mu \mathrm{m}$ in the square analysis area with a size of $L=300 \mu \mathrm{m}$ [the 3-D saturation amplitude ${ }^{46,21}$ for the $\lambda=60-\mu \mathrm{m}$ wavelength is $\left.S_{3-\mathrm{D}}=\lambda^{2} /\left(2 \pi^{2} L\right) \cong 0.3 \mu \mathrm{m}\right]$. All measurements were performed $1.0 \mathrm{~ns}$ after the beginning of the drive, when the growth of imprinted and preimposed modulations was similar. All three types of target modulations (2-D imprinted, 2-D preimposed, and 3-D imprinted) at $60-\mu \mathrm{m}$ wavelength grow with similar growth rates for each shot, as shown in Fig. 98.32. The solid lines in Fig. 98.32 show exponential fits to the experimental data. The inferred growth rates were $1.7 \pm 0.2 \mathrm{~ns}^{-1}, 1.5 \pm 0.2 \mathrm{~ns}^{-1}$, and $1.6 \pm 0.2 \mathrm{~ns}^{-1}$ for the 2-D imprinted, 2-D preimposed, and 3-D imprinted modulations, respectively, for the shot without SSD. The corresponding growth rates for the shot with SSD are $1.7 \pm 0.3 \mathrm{~ns}^{-1}, 2.0 \pm$ $0.3 \mathrm{~ns}^{-1}$, and $2.1 \pm 0.3 \mathrm{~ns}^{-1}$.
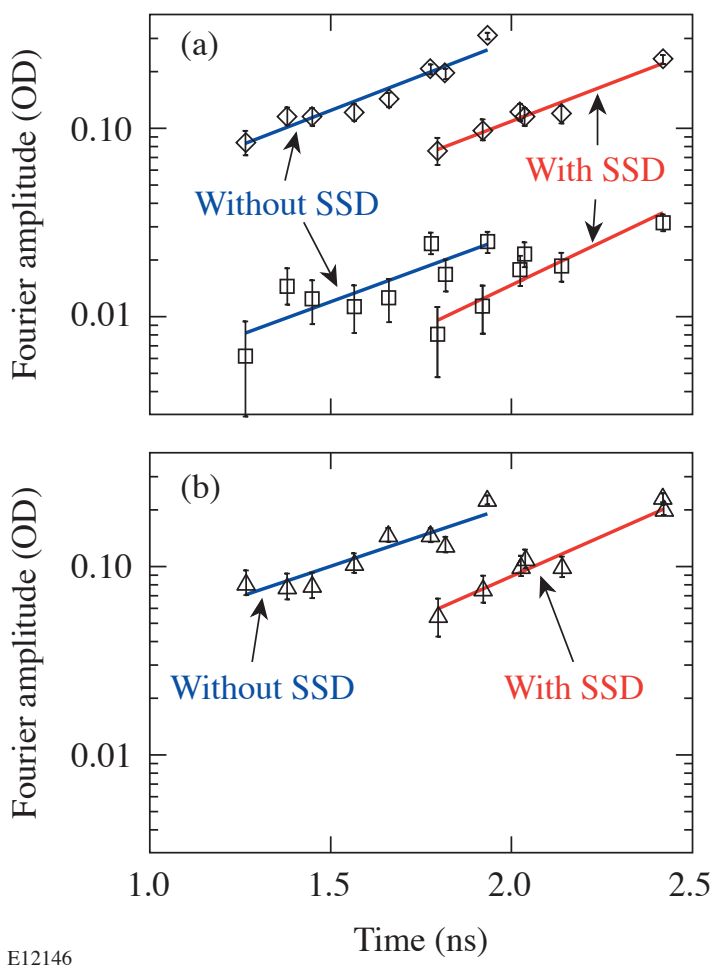

Figure 98.32

Fourier amplitudes of optical-depth modulations as a function of time for (a) 2-D imprinted modulations (diamonds, upper data), 3-D broadband modulations (squares, lower data), and (b) the 2-D preimposed modulations (triangles). Data with SSD comes later than the data without SSD. 
The surface equivalent amplitude of an imprinted 2-D, $60-\mu \mathrm{m}$-wavelength perturbation from a $48^{\circ}$ beam was $\eta_{\text {imp 2-D }}(t=0)=0.16 \pm 0.01 \mu \mathrm{m}$, from Eq. (2). The initial modulation amplitude of $2-\mathrm{D}$ preimposed modulation $\eta_{\text {pre 2-D }}(t=0)=0.125 \mu \mathrm{m}$, and the ratios of measured opticaldepth modulations at various times,

$$
\delta\left[\rho R_{\text {imp 2-D }}(k, t)\right] / \delta\left[\rho R_{\text {pre 2-D }}(k, t)\right],
$$

taken from Figs. 98.32(a) and 98.32(b). The surface equivalent amplitude of 3-D imprinted, $60-\mu \mathrm{m}$-wavelength perturbations from $23^{\circ}$ beams was $\eta_{\text {imp 3-D }}(t=0)=0.016 \pm 0.001 \mu \mathrm{m}$. The imprint efficiencies at $60-\mu \mathrm{m}$ wavelength for $48^{\circ}$ and $23^{\circ}$ beams were $E_{48^{\circ}}=2.5 \pm 0.2 \mu \mathrm{m}$ and $E_{23^{\circ}}=3.0 \pm 0.3 \mu \mathrm{m}$, respectively, as calculated using Eq. (3) and relative laser modulations $[\delta I(k) / I]_{48^{\circ}}=6.3 \pm 0.4 \%$ for the $48^{\circ}$ beam and $\left[\delta I(k) / I_{23^{\circ}}=0.54 \pm 0.04 \%\right.$ for the $23^{\circ}$ beams at a spatial wavelength of $60 \mu \mathrm{m}$. As shown in Ref. 43, the imprint efficiency for more-oblique beams is lower because these beams see an effectively longer length of plasma on the way to the ablation surface than the less-oblique beams; therefore, plasma smoothing is more effective for larger-angle-of-incidence beams. ${ }^{43}$ Figure 98.32 (a) shows the effect of SSD on imprint reduction. The imprinting amplitudes (of $23^{\circ}$ and $48^{\circ}$ beams) are reduced by a factor of $\sim 2.5$ at a spatial wavelength of $60 \mu \mathrm{m}$.

\section{Conclusions}

The first measurements of imprint efficiency for laser beams incident at two different angles $\left(23^{\circ}\right.$ and $\left.48^{\circ}\right)$ to a target normal have been presented. The measurements were performed at a spatial wavelength of $60 \mu \mathrm{m}$ with and without smoothing by spectral dispersion (SSD). The imprinted amplitudes were calibrated with preimposed, 60- $\mu \mathrm{m}$-wavelength perturbations during the linear phase of RT growth. The measured imprint efficiencies at the spatial wavelength of $60 \mu \mathrm{m}$ were $2.5 \pm 0.2 \mu \mathrm{m}$ for the beam with a $48^{\circ}$ angle of incidence and $3.0 \pm 0.3 \mu \mathrm{m}$ for the beams with a $23^{\circ}$ angle of incidence. The SSD reduced modulations by a factor of $\sim 2.5$ at the same spatial wavelength.

\section{ACKNOWLEDGMENT}

This work was supported by the U.S. Department of Energy Office of Inertial Confinement Fusion under Cooperative Agreement No. DE-FC0392SF19460, the University of Rochester, and the New York State Energy Research and Development Authority. The support of DOE does not constitute an endorsement by DOE of the views expressed in this article.

\section{REFERENCES}

1. J. Nuckolls et al., Nature 239, 139 (1972).

2. S. E. Bodner, D. G. Colombant, J. H. Gardner, R. H. Lehmberg, S. P. Obenschain, L. Phillips, A. J. Schmitt, J. D. Sethian, R. L. McCrory, W. Seka, C. P. Verdon, J. P. Knauer, B. B. Afeyan, and H. T. Powell, Phys. Plasmas 5, 1901 (1998).

3. J. D. Lindl, Inertial Confinement Fusion: The Quest for Ignition and Energy Gain Using Indirect Drive (Springer-Verlag, New York, 1998), Chap. 6, pp. 61-82.

4. M. H. Emery et al., Phys. Fluids B 3, 2640 (1991).

5. M. Desselberger et al., Phys. Rev. Lett. 68, 1539 (1992).

6. M. Desselberger et al., Phys. Rev. Lett. 74, 2961 (1995).

7. M. Dunne et al., Phys. Rev. Lett. 75, 3858 (1995).

8. R. J. Taylor et al., Phys. Rev. Lett. 76, 1643 (1996).

9. D. H. Kalantar, M. H. Key, L. B. Da Silva, S. G. Glendinning, J. P. Knauer, B. A. Remington, F. Weber, and S. V. Weber, Phys. Rev. Lett. 76, 3574 (1996).

10. S. G. Glendinning, S. N. Dixit, B. A. Hammel, D. H. Kalantar, M. H. Key, J. D. Kilkenny, J. P. Knauer, D. M. Pennington, B. A. Remington, R. J. Wallace, and S. V. Weber, Phys. Rev. E 54, 4473 (1996).

11. R. G. Watt, D. C. Wilson, R. E. Chrien, R. V. Hollis, P. L. Gobby, R. J. Mason, R. A. Kopp, R. A. Lerche, D. H. Kalantar, B. MacGowan, M. B. Nelson, T. Phillips, P. W. McKenty, and O. Willi, Phys. Plasmas 4, 1379 (1997).

12. R. J. Taylor et al., Phys. Rev. Lett. 79, 1861 (1997).

13. C. J. Pawley et al., Phys. Plasmas 4, 1969 (1997).

14. S. V. Weber, S. G. Glendinning, D. H. Kalantar, M. H. Key, B. A. Remington, J. E. Rothenberg, E. Wolfrum, C. P. Verdon, and J. P. Knauer, Phys. Plasmas 4, 1978 (1997).

15. D. H. Kalantar, M. H. Key, L. B. Da Silva, S. G. Glendinning, B. A. Remington, J. E. Rothenberg, F. Weber, S. V. Weber, E. Wolfrum, N. S. Kim, D. Neely, J. Zhang, J. S. Wark, A. Demir, J. Lin, R. Smith, G. J. Tallents, C. L. S. Lewis, A. MacPhee, J. Warwick, and J. P. Knauer, Phys. Plasmas 4, 1985 (1997).

16. H. Azechi et al., Phys. Plasmas 4, 4079 (1997).

17. E. Wolfrum et al., Phys. Plasmas 5, 227 (1988).

18. A. L. Velikovich et al., Phys. Plasmas 5, 1491 (1998).

19. S. G. Glendinning, S. N. Dixit, B. A. Hammel, D. H. Kalantar, M. H. Key, J. D. Kilkenny, J. P. Knauer, D. M. Pennington, B. A. Remington, J. Rothenberg, R. J. Wallace, and S. V. Weber, Phys. Rev. Lett. 80, 1904 (1998). 
20. R. G. Watt, J. Duke, C. J. Fontes, P. L. Gobby, R. V. Hollis, R. A. Kopp, R. J. Mason, D. C. Wilson, C. P. Verdon, T. R. Boehly, J. P. Knauer, D. D. Meyerhofer, V. Smalyuk, R. P. J. Town, A. Iwase, and O. Willi, Phys. Rev. Lett. 81, 4644 (1998).

21. V. A. Smalyuk, T. R. Boehly, D. K. Bradley, V. N. Goncharov, J. A. Delettrez, J. P. Knauer, D. D. Meyerhofer, D. Oron, and D. Shvarts, Phys. Rev. Lett. 81, 5342 (1998).

22. V. A. Smalyuk, T. R. Boehly, D. K. Bradley, V. N. Goncharov, J. A. Delettrez, J. P. Knauer, D. D. Meyerhofer, D. Oron, D. Shvarts, Y. Srebro, and R. P. J. Town, Phys. Plasmas 6, 4022 (1999).

23. D. G. Colombant et al., Phys. Plasmas 7, 2046 (2000).

24. V. N. Goncharov, S. Skupsky, T. R. Boehly, J. P. Knauer, P. McKenty, V. A. Smalyuk, R. P. J. Town, O. V. Gotchev, R. Betti, and D. D. Meyerhofer, Phys. Plasmas 7, 2062 (2000).

25. A. J. Schmitt et al., Phys. Plasmas 8, 2287 (2001).

26. T. R. Boehly, V. N. Goncharov, O. Gotchev, J. P. Knauer, D. D. Meyerhofer, D. Oron, S. P. Regan, Y. Srebro, W. Seka, D. Shvarts, S. Skupsky, and V. A. Smalyuk, Phys. Plasmas 8, 2331 (2001).

27. M. Nishikino et al., Phys. Plasmas 9, 1381 (2002).

28. M. Nakai et al., Phys. Plasmas 9, 1734 (2002).

29. S. P. Obenschain et al., Phys. Plasmas 9, 2234 (2002).

30. T. Endo et al., Phys. Rev. Lett. 74, 3608 (1995).

31. R. Ishizaki and K. Nishihara, Phys. Rev. Lett. 78, 1920 (1997).

32. V. N. Goncharov, Phys. Rev. Lett. 82, 2091 (1999).

33. Y. Lin, T. J. Kessler, and G. N. Lawrence, Opt. Lett. 20, 764 (1995).

34. T. R. Boehly, V. A. Smalyuk, D. D. Meyerhofer, J. P. Knauer, D. K. Bradley, R. S. Craxton, M. J. Guardalben, S. Skupsky, and T. J. Kessler, J. Appl. Phys. 85, 3444 (1999).
35. S. P. Regan, J. A. Marozas, J. H. Kelly, T. R. Boehly, W. R. Donaldson, P. A. Jaanimagi, R. L. Keck, T. J. Kessler, D. D. Meyerhofer, W. Seka, S. Skupsky, and V. A. Smalyuk, J. Opt. Soc. Am. B 17, 1483 (2000).

36. T. R. Boehly, D. L. Brown, R. S. Craxton, R. L. Keck, J. P. Knauer, J. H. Kelly, T. J. Kessler, S. A. Kumpan, S. J. Loucks, S. A. Letzring, F. J. Marshall, R. L. McCrory, S. F. B. Morse, W. Seka, J. M. Soures, and C. P. Verdon, Opt. Commun. 133, 495 (1997).

37. R. H. Lehmberg and S. P. Obenschain, Opt. Commun. 46, 27 (1983).

38. H. Nakano et al., J. Appl. Phys. 73, 2122 (1993).

39. Y. Aglitskiy et al., Phys. Rev. Lett. 87, 265001 (2001).

40. Y. Aglitskiy et al., Phys. Rev. Lett. 87, 265002 (2001).

41. Y. Aglitskiy et al., Phys. Plasmas 9, 2264 (2002).

42. R. L. McCrory and C. P. Verdon, in Computer Applications in Plasma Science and Engineering, edited by A. T. Drobot (Springer-Verlag, New York, 1991), Chap. 11, pp. 291-325.

43. V. A. Smalyuk, J. A. Delettrez, S. B. Dumanis, R. Epstein, V. Yu. Glebov, D. D. Meyerhofer, P. B. Radha, T. C. Sangster, C. Stoeckl, N. C. Toscano, J. A. Frenje, C. K. Li, R. D. Petrasso, F. H. Séguin, and J. H. Koch, "Hot-Core Characterization of the Cryogenic $\mathrm{D}_{2}$ Target at Peak Neutron Production in Direct-Drive Spherical Implosion," to be submitted to Physical Review Letters.

44. V. A. Smalyuk, T. R. Boehly, D. K. Bradley, J. P. Knauer, and D. D. Meyerhofer, Rev. Sci. Instrum. 70, 647 (1999).

45. J. Delettrez, R. Epstein, M. C. Richardson, P. A. Jaanimagi, and B. L. Henke, Phys. Rev. A, Gen. Phys. 36, 3926 (1987).

46. M. J. Dunning and S. W. Haan, Phys. Plasmas 2, 1669 (1995). 


\section{Stopping of Directed Energetic Electrons in High-Temperature Hydrogenic Plasmas}

A basic problem in plasma physics is the interaction and energy loss of energetic charged particles in plasmas. ${ }^{1-4}$ This problem has traditionally focused on ions (i.e., protons, alphas, etc.), either in the context of heating and/or ignition in, for example, inertial confinement fusion (ICF) ${ }^{3-6}$ or the use of these particles for diagnosing implosion dynamics. ${ }^{7}$ More recently, prompted in part by the concept of fast ignition for $\mathrm{ICF},{ }^{8}$ scientists have begun considering energy deposition from relativistic fast electrons in deuterium-tritium (DT) plasmas. ${ }^{8-13}$ Tabak et al. ${ }^{8}$ used, for example, the energy deposition of Berger and Seltzer, ${ }^{14}$ which is based on the continuous slowing down of electrons in cold matter. This treatment, though quite similar to electrons slowing in plasmas, does not include the effects of scattering. Deutsch et al. ${ }^{9}$ addressed this issue by considering the effects of scattering off the background ions; ${ }^{16,17}$ they ignored scattering due to background electrons.

In another important context in ICF, researchers addressed the issue of fuel preheat due to energetic electrons $(\sim 50$ to $300 \mathrm{keV}), 5,18,19$ the consequence of which is to elevate the fuel adiabat to levels that would prohibit ignition. This article shows that scattering effects could be significant for quantitative evaluations of preheat.

The starting point for these calculations is the relativistic elastic differential cross sections for electrons scattering off fully ionized ions of charge $Z$ (Refs. 20-22) and off the neutralizing bath of electrons, ${ }^{21,23,24}$ which are approximated as

$$
\begin{gathered}
\left(\frac{d \sigma}{d \Omega}\right)^{\text {ei }} \approx \frac{Z^{2}}{4}\left(\frac{r_{0}}{\gamma \beta^{2}}\right)^{2} \frac{1}{\sin ^{4} \theta / 2}, \\
Z\left(\frac{d \sigma}{d \Omega}\right)^{\text {ee }} \approx Z \frac{(\gamma+1)^{2}}{\left(2^{\sqrt{(\gamma+1) / 2}}\right)^{4}}\left(\frac{r_{0}}{\gamma \beta^{2}}\right)^{2} \frac{1}{\sin ^{4} \theta / 2},
\end{gathered}
$$

where $\beta=v / c, \gamma=\left(1-\beta^{2}\right)^{-1 / 2}$, and $r_{0}=e^{2} / m_{0} c^{2}$ is the classical electron radius. The relative importance of electron scattering is implied from the ratio

$$
\Re=Z\left(\frac{d \sigma}{d \Omega}\right)^{\text {ee }} /\left(\frac{d \sigma}{d \Omega}\right)^{\text {ei }} \approx \frac{4(\gamma+1)^{2}}{\left(2^{\sqrt{(\gamma+1) / 2}}\right)^{4}} \frac{1}{Z} .
$$

For a hydrogenic plasma $(Z=1)$ and for $\gamma \leqslant 10, \Re \sim 1$, indicating that the electron component is equally important. As best we can tell, the electron-scattering component has been largely ignored since it was typically assumed, usually justifiably, that ion scattering dominates. This will not be the case, however, for problems discussed here, for relativistic astrophysical jets ${ }^{25}$ or for many of the present high-energy laserplasma experiments ${ }^{26}$ for which $Z \sim 1$ and $\gamma \lesssim 10$.

To calculate the effects of multiple scattering, a Boltzmannlike diffusion equation is used:27

$$
\frac{\partial f}{\partial s}+\mathbf{v} \cdot \nabla f=n_{i} \int\left[f\left(\mathbf{x}, \mathbf{v}^{\prime}, s\right)-f(\mathbf{x}, \mathbf{v}, s)\right] \sigma\left(\left|\mathbf{v}-\mathbf{v}^{\prime}\right|\right) d \mathbf{v}^{\prime},
$$

where $f$ is the angular distribution function of the scattered electrons, $n_{i}$ is the number density of plasma ions of charge $Z$, $\mathbf{x}$ is the position where scattering occurs, and $\sigma=\sigma_{\mathrm{ei}}+Z \sigma_{\mathrm{ee}}$ is the total scattering cross section, where $\sigma_{\mathrm{ei}}=\int(d \sigma / d \Omega)^{\mathrm{ei}} d \Omega$ and $\sigma_{\mathrm{ee}}=\int(d \sigma / d \Omega)^{\mathrm{ee}} d \Omega$. Equation (4) is solved in cylindrical coordinates with the assumption that the scattering is azimuthally symmetric. The solution that satisfies the boundary conditions is ${ }^{27,28}$

$$
f(\theta, s)=\frac{1}{4 \pi} \sum_{\ell=0}^{\infty}(2 \ell+1) P_{\ell}(\cos \theta) \exp \left[-\int_{0}^{s} \sigma_{\ell}\left(s^{\prime}\right) d s^{\prime}\right],
$$


where $P_{\ell}(\cos \theta)$ is a Legendre polynomial. Using orthogonality and projecting the $\ell=1$ term,

$$
\begin{aligned}
\langle\cos \theta\rangle & =\int f(\theta, s) P_{1}(\cos \theta) d \Omega=\exp \left[-\int_{0}^{s} \sigma_{1}\left(s^{\prime}\right) d s^{\prime}\right] \\
& =\exp \left[-\int_{E_{0}}^{E} \sigma_{1}(E)\left(\frac{d E}{d s}\right)^{-1} d E\right],
\end{aligned}
$$

where $\langle\cos \theta\rangle$, a function of the residual electron energy, is a measure of the mean deflection resulting from multiple scattering, ${ }^{29}$ and relates $d E / d s$ to $d E / d x$ through

$$
\frac{d E}{d x}=\langle\cos \theta\rangle^{-1} \frac{d E}{d s},
$$

where $d E / d s$ is the stopping power along the path while $d E / d x$ is the linear energy stopping power. In the above,

$$
S(E)=\int_{0}^{s} d s^{\prime}=\int_{E_{0}}^{E}\left(\frac{d E}{d s}\right)^{-1} d E
$$

and

$$
\sigma_{1}(E)=2 \pi n_{i} \int_{0}^{\pi}\left(\frac{d \sigma}{d \Omega}\right)(1-\cos \theta) \sin \theta d \theta,
$$

where $\sigma_{1}$ is the diffusion cross section (or transport cross section) that characterizes the loss of directed electron velocity through scattering. ${ }^{2}$ Equations (1) and (2) are substituted into Eq. (9), and, after a standard change of variables, the integrations are taken from $b_{\min }^{\mathrm{ei}}$ or $b_{\min }^{\mathrm{ee}}$ to $\lambda_{D}$, where $\lambda_{D}$ is the Debye length, ${ }^{30}$ and $b_{\min }^{\mathrm{ei}}\left(b_{\mathrm{min}}^{\mathrm{ee}}\right)$ is the larger of $b_{\text {quantum }}^{\text {ei }}\left(b_{\text {quantum }}^{\text {ee }}\right)$ and $b_{\perp}^{\mathrm{ei}}\left(b_{\perp}^{\mathrm{ee}}\right)$ (Ref. 31$)$. $b_{\text {quantum }}^{\text {ei }}$ and $b_{\text {quantum }}^{\text {ee }}$ are approximately the electron deBroglie wavelength, and $b_{\perp}^{\mathrm{ei}}=Z r_{0} / \gamma \beta^{2}$ and

$$
b_{\perp}^{\mathrm{ee}} \approx 2(\gamma+1) r_{0} /\left[\left(2^{\sqrt{(\gamma+1) / 2}}\right)^{2} \gamma \beta^{2}\right]
$$

are the impact parameters for $90^{\circ}$ scattering of electrons off ions $(e \rightarrow i)$ or electrons off electrons $(e \rightarrow e)$. Thus

$$
\begin{aligned}
\sigma_{1}(E) & =\sigma_{1}^{\mathrm{ei}}(E)+Z \sigma_{1}^{\mathrm{ee}}(E) \\
& =4 \pi n_{i}\left(\frac{r_{0}}{\gamma \beta^{2}}\right)^{2}\left[Z^{2} \ln \Lambda^{\mathrm{ei}}+\frac{4(\gamma+1)^{2}}{\left(2^{\sqrt{(\gamma+1) / 2}}\right)^{4}} Z \ln \Lambda^{\mathrm{ee}}\right],
\end{aligned}
$$

where the arguments of the Coulomb logarithm are $\Lambda^{\mathrm{ei}}=\lambda_{\mathrm{D}} / b_{\min }^{\mathrm{ei}}$ and $\Lambda^{\mathrm{ee}}=\lambda_{\mathrm{D}} / b_{\min }^{\mathrm{ee}}$ (Ref. 29). Since these Coulomb logarithms are used in this and later calculations, they are shown in Fig. 98.33.

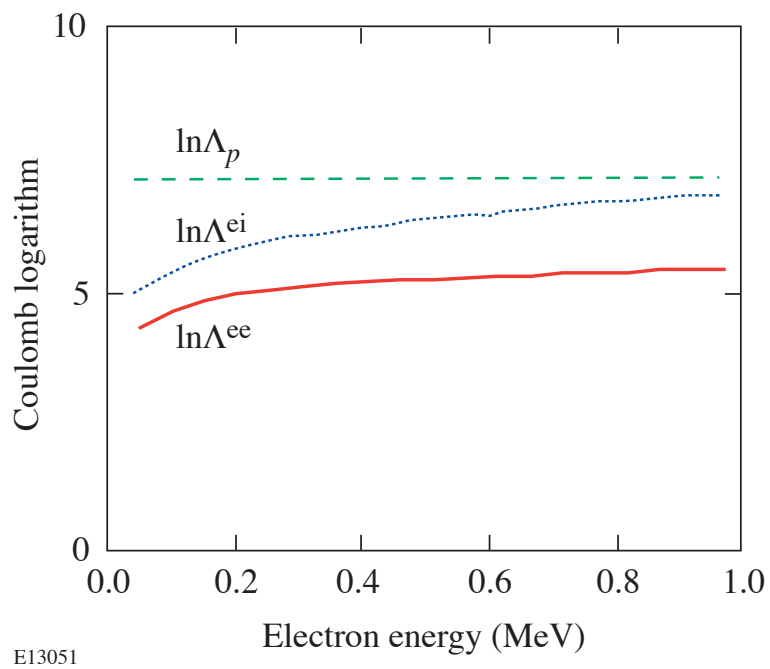

Figure 98.33

The Coulomb logarithms for incident $1-\mathrm{MeV}$ electrons interacting with a DT plasma $\left(\rho=300 \mathrm{~g} / \mathrm{cm}^{3} ; T_{e}=5 \mathrm{keV}\right)$. For the background plasma, the Coulomb $\log$ arithm $\ln \Lambda_{p}$, relevant to plasma transport processes (e.g., electrical and thermal conductivity), is about 7 .

The stopping power in Eq. (6) consists of contributions from binary interactions with plasma electrons and from plasma oscillations. The binary contribution is ${ }^{32}$

$$
\left(\frac{d E}{d s}\right)_{b}=-n_{i} Z(\gamma-1) m_{0} c^{2} \int_{\varepsilon_{\min }}^{\varepsilon_{\max }} \varepsilon\left(\frac{d \sigma}{d \varepsilon}\right) d \varepsilon,
$$


where the differential energy loss cross section is from ${\mathrm{M} ø l l e{ }^{23}}^{23}$

$$
\begin{aligned}
\frac{d \sigma}{d \varepsilon}= & \frac{2 \pi r_{0}^{2}}{(\gamma-1) \beta^{2}} \\
& \times\left[\frac{1}{\varepsilon^{2}}+\frac{1}{(1-\varepsilon)^{2}}+\left(\frac{\gamma-1}{\gamma}\right)^{2}-\frac{2 \gamma-1}{\gamma^{2} \varepsilon(1-\varepsilon)}\right],
\end{aligned}
$$

and $\varepsilon$ is the energy transfer in units of $(\gamma-1) m_{0} c^{2}$. The lower integration limit reflects the minimum energy transfer that occurs when an incident electron interacts with a plasma electron at $\lambda_{D}$, i.e., $\varepsilon_{\min }=2 \gamma r_{0}^{2} /\left[\lambda_{\mathrm{D}}(\gamma-1)\right]^{2}$. The upper limit occurs for a head-on collision, for which $\varepsilon_{\max }=0.5$.

The contribution from plasma oscillations, which reflects the response of the plasma to impact parameters larger than $\lambda_{D},{ }^{31}$ is

$$
\left(\frac{d E}{d s}\right)_{c}=-\frac{4 \pi r_{0}^{2} m_{0} c^{2} n_{i} Z}{\beta^{2}} \ln \left(\frac{1.123 \beta}{\sqrt{2 k T_{e} / m_{0} c^{2}}}\right),
$$

where relativistic effects are included. Consequently,

$$
\begin{aligned}
\frac{d E}{d s}= & -\frac{2 \pi r_{0}^{2} m_{0} c^{2} n_{i} Z}{\beta^{2}}\left\{\ln \left[\frac{(\gamma-1) \lambda_{\mathrm{D}}}{2 \sqrt{2 \gamma} r_{0}}\right]^{2}+1+\frac{1}{8}\left(\frac{\gamma-1}{\gamma}\right)^{2}\right. \\
& \left.-\left(\frac{2 \gamma-1}{\gamma}\right) \ln 2+\ln \left(\frac{1.123 \beta}{\sqrt{2 k T_{e} / m_{0} c^{2}}}\right)^{2}\right\} .
\end{aligned}
$$

Figure 98.34 illustrates this relationship [Eq. (6)], where the incident electron $\left(E_{0}=1 \mathrm{MeV}\right)$ continuously changes direction as it loses energy. When $\langle\cos \theta\rangle$ equals one $e$-folding, $|\theta| \approx 68^{\circ}$ and $E / E_{0} \approx 0.1$, at which point the incident electron has lost memory of its initial direction.

We iterate upon this process, important for low-energy electrons, until the electrons are thermalized with the background plasma, which has the cumulative effect of bending the path of the electrons away from their initial direction. Figure 98.35 illustrates the enhancement of $d E / d x$ for scattering off ions and for scattering off ions plus electrons.

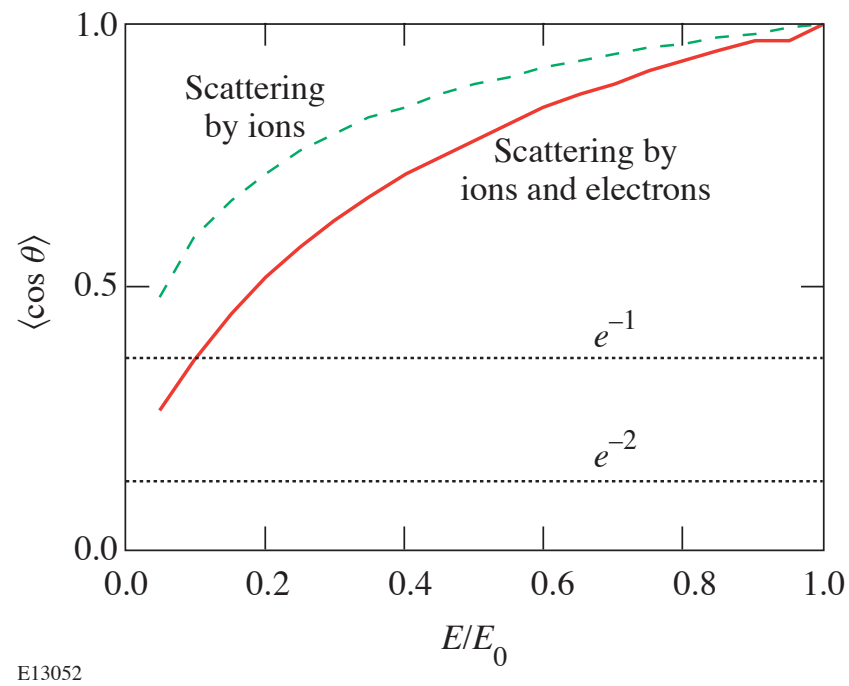

Figure 98.34

The mean deflection angle $\langle\cos \theta\rangle$ is plotted against the fraction of the residual energy in a DT plasma for $e \rightarrow i$ and for $e \rightarrow i+e$ scattering ( $1-\mathrm{MeV}$ electrons with $\left.\rho=300 \mathrm{~g} / \mathrm{cm}^{3} ; T_{e}=5 \mathrm{keV}\right)$. When $\langle\cos \theta\rangle$ equals one $e$-folding, corresponding to $|\theta| \approx 68^{\circ}$ and $E / E_{0} \approx 0.1$, the incident electron has lost memory of its initial direction.

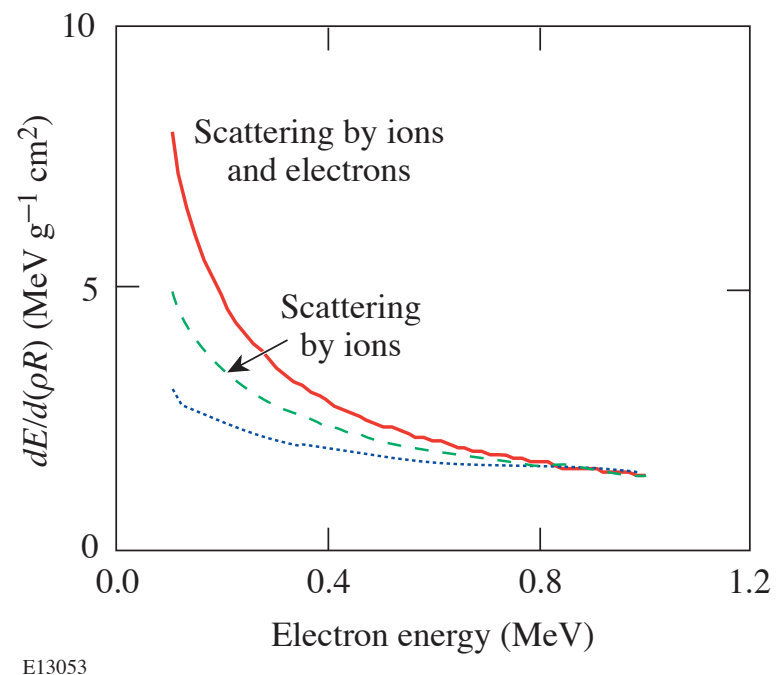

Figure 98.35

Stopping power for linear-energy transfer and continuous slowing down are plotted as functions of the electron energy for incident $1-\mathrm{MeV}$ electrons in a DT plasma $\left(\rho=300 \mathrm{~g} / \mathrm{cm}^{3} ; T_{e}=5 \mathrm{keV}\right)$. Enhancement of $d E / d x$ (solid line) over $d E / d s$ (dotted line) is a consequence of the effects of multiple scattering. 
This effect is further illustrated in Fig. 98.36, where the corresponding set of curves for range $(R)$ and penetration $\left(\left\langle X_{p}\right\rangle\right)$ with and without the electron scattering contributions are shown for electrons with $E_{0}=0.1-10 \mathrm{MeV}$.

$$
R=\int_{0}^{R} d s^{\prime}=\int_{E_{0}}^{\sim k T}\left(\frac{d E}{d s}\right)^{-1} d E
$$

and

$$
\left\langle X_{p}\right\rangle \approx \sum_{n=0} e^{-n} \int_{E_{n}}^{E_{n+1}}\langle\cos \theta\rangle\left(\frac{d E}{d s}\right)^{-1} d E,
$$

where $E_{0}$ is the initial energy; $E_{1}, E_{2} \ldots$ correspond to the electron energies at the first, second.... $e$-folding of $\langle\cos \theta\rangle$ (see Fig. 98.34); $R$ is the total path length the electron traverses as it scatters about and eventually thermalizes; and $\left\langle X_{p}\right\rangle$ is the distance along the initial electron trajectory that it eventually reaches. Contributions from electron and ion scattering are shown in Fig. 98.36.
Three other points are worth noting: First, the temperature and density dependence are weak, i.e., a factor-of-10 reduction in either temperature or density results in only $\sim 10 \%$ reduction in the penetration. Second, as the initial electron energy decreases, the effects of scattering become more pronounced [Fig. 98.36(c)] - an effect, very similar in nature, that is also seen in the scattering of energetic electrons in metals. ${ }^{34}$ Third, for a given electron energy, scattering effects decrease slightly as the target plasma temperature decreases, i.e., the path of the electron straightens slightly as the target plasma temperature drops. For example, when the target plasma temperature changes from 5.0 to $0.5 \mathrm{keV}\left(\rho=300 \mathrm{~g} / \mathrm{cm}^{3}\right)$, the ratio $R /\left\langle X_{p}\right\rangle$ is reduced by $\sim 5 \%$ for $1-\mathrm{MeV}$ electrons.

By calculating of the penetration as a function of energy loss, the energy deposition can be evaluated (Fig. 98.37). In addition to the differences in total penetration with and without scattering contributions, it is seen that the linear-energy transfer increases near the end of its penetration (i.e., an effective Bragg peak), an effect that is seen more weakly with just ion scattering. Such differences may need to be considered in quantitatively modeling the energy deposition of relativistic electrons for fast ignition and for critically assessing ignition requirements. ${ }^{35}$ It is also interesting, and a consequence of

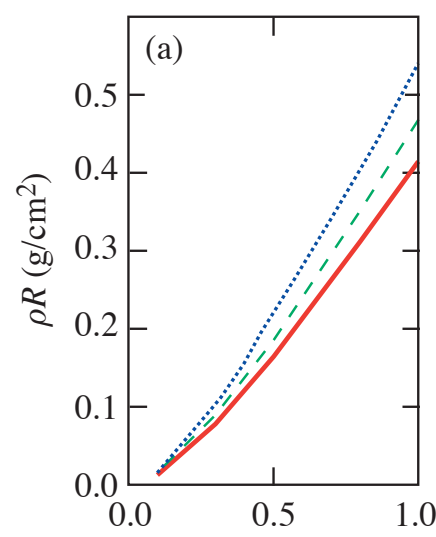

Electron energy $(\mathrm{MeV})$

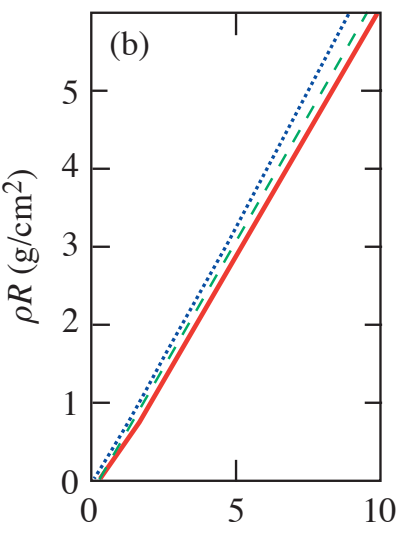

Electron energy $(\mathrm{MeV})$

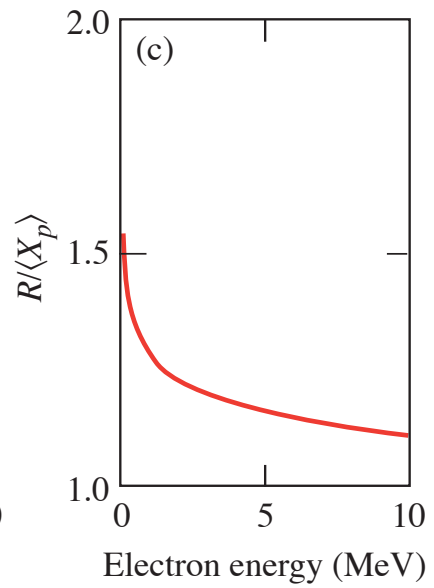

Electron energy $(\mathrm{MeV})$

E13054

\begin{tabular}{l}
\hline ......... Continuous slowing down \\
--- Scattering by ions \\
- Scattering by ions and electrons
\end{tabular}

Figure 98.36

The range (dotted line) and penetration for (a) 0.1 - to $1-\mathrm{MeV}$ electrons and (b) 1 - to $10-\mathrm{MeV}$ electrons in a DT plasma $\left(\rho=300 \mathrm{~g} / \mathrm{cm}^{3} ; T_{e}=5 \mathrm{keV}\right)$. The penetration is shown for scattering off ions and for scattering off ions plus electrons. A factor-of-10 reduction in either the temperature or density results in only $\sim 10 \%$ reduction in the penetration. (c) The ratio of range to penetration for $0.1-$ to $10-\mathrm{MeV}$ electrons. As the initial electron energy decreases, the effects of multiple scattering become more pronounced, and the penetration is further diminished with respect to the range. 
selecting 1-MeV electrons [Figs. 98.36 and 98.37], that the effects of scattering reduce the penetration from 0.54 to $0.41 \mathrm{~g} / \mathrm{cm}^{2}$; this latter value is close to the range of $3.5-\mathrm{MeV}$ alphas, $0.3 \mathrm{~g} / \mathrm{cm}^{2}$, which is required for hot-spot ignition in a $10-\mathrm{keV}$ plasma. ${ }^{3-6}$

Finally, in order to explore the importance of electron-onelectron multiple scattering in a hydrogenic setting, and since definitive stopping power experiments in plasmas are extremely difficult, we propose that experiments be undertaken in which a monoenergetic electron beam, with energy between 0.1 and $1.0 \mathrm{MeV}$, scatters off thin layers of either $\mathrm{D}_{2}$ or $\mathrm{H}_{2}$ ice, where the thickness of the ice layer is between $\sim 100$ and $1000 \mu \mathrm{m}$, the appropriate thickness depending on the exact electron energy. Although there are differences in the scattering calculations for cold, condensed hydrogenic matter and a hydrogenic plasma, there is reason to believe that the relative importance of the electron-to-electron and the electron-to-ion multiple scattering terms will be approximately the same for both states of matter.

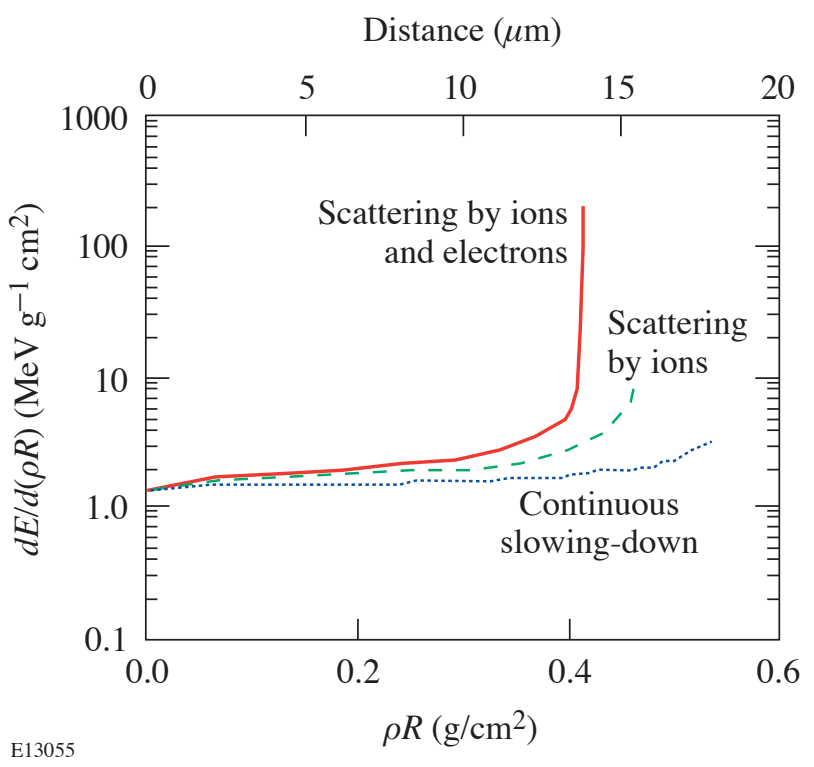

Figure 98.37

The stopping power for $1-\mathrm{MeV}$ electrons, plotted as a function of the electron penetration, for a DT plasma with $\rho=300 \mathrm{~g} / \mathrm{cm}^{3}$ and $T_{e}=5 \mathrm{keV}$. The three curves correspond to three different models. As a result of the scattering effects, the energy transfer increases notably near the end of the penetration (i.e., an effective Bragg peak). For these $1-\mathrm{MeV}$ electrons, the effects of scattering reduce the penetration from $0.54 \mathrm{~g} / \mathrm{cm}^{2}$ to $0.41 \mathrm{~g} / \mathrm{cm}^{2}$ (Ref. 33).

\section{Summary}

The energy loss and penetration of energetic electrons into a hydrogenic plasma has been analytically calculated, and the effect of scattering off ions and electrons is treated from a unified point of view. In general, scattering enhances the electron linear-energy transfer along the initial electron direction and reduces the electron penetration. Energy deposition increases near the end of its range. These results should have relevance to "fast ignition" and to fuel preheat in inertial confinement fusion, specifically to energy deposition calculations that critically assess quantitative ignition conditions.

\section{ACKNOWLEDGMENT}

This work was supported in part by the U.S. Department of Energy Contract \#DE-FG03-99SF21782, LLE subcontract \#PO410025G, LLNL subcontract \#B313975.

\section{REFERENCES}

1. L. Spitzer, Physics of Fully Ionized Gases, 2nd rev. ed., Interscience Tracts on Physics and Astronomy (Interscience, New York, 1962).

2. B. Trubnikov, Review of Plasma Physics (Consultants Bureau, New York, 1965).

3. S. Skupsky, Phys. Rev. A, 16, 727 (1977); G. S. Fraley et al., Phys. Fluids 17, 474 (1974).

4. C. K. Li and R. D. Petrasso, Phys. Rev. Lett. 70, 3059 (1993).

5. J. D. Lindl, Inertial Confinement Fusion: The Quest for Ignition and Energy Gain Using Indirect Drive (Springer-Verlag, New York, 1998); references therein.

6. J. D. Lindl, R. L. McCrory, and E. M. Campbell, Phys. Today 45, 32 (1992).

7. R. D. Petrasso, J. A. Frenje, C. K. Li, F. H. Séguin, J. R. Rygg, B. E. Schwartz, S. Kurebayashi, P. B. Radha, C. Stoeckl, J. M. Soures, J. Delettrez, V. Yu. Glebov, D. D. Meyerhofer, and T. C. Sangster, Phys. Rev. Lett. 90, 095002 (2003); V. A. Smalyuk, P. B. Radha, J. A. Delettrez, V. Yu. Glebov, V. N. Goncharov, D. D. Meyerhofer, S. P. Regan, S. Roberts, T. C. Sangster, J. M. Soures, C. Stoeck1, J. A. Frenje, C. K. Li, R. D. Petrasso, and F. H. Séguin, Phys. Rev. Lett. 90, 135002 (2003); F. H. Séguin, J. A. Frenje, C. K. Li, D. G. Hicks, S. Kurebayashi, J. R. Rygg, B.-E. Schwartz, R. D. Petrasso, S. Roberts, J. M. Soures, D. D. Meyerhofer, T. C. Sangster, J. P. Knauer, C. Sorce, V. Yu. Glebov, C. Stoeckl, T. W. Phillips, R. J. Leeper, K. Fletcher, and S. Padalino, Rev. Sci. Instrum. 74, 975 (2003).

8. M. Tabak et al., Phys. Plasmas 1, 1626 (1994).

9. C. Deutsch et al., Phys. Rev. Lett. 77, 2483 (1996); ibid. 85, 1140 (2000).

10. S. Atzeni, Phys. Plasmas 6, 3316 (1999). 
11. M. H. Key, M. D. Cable, T. E. Cowan, K. G. Estabrook, B. A. Hammel, S. P. Hatchett, E. A. Henry, D. E. Hinkel, J. D. Kilkenny, J. A. Koch, W. L. Kruer, A. B. Langdon, B. F. Lasinski, R. W. Lee, B. J. MacGowan, A. MacKinnon, J. D. Moody, M. J. Moran, A. A. Offenberger, D. M. Pennington, M. D. Perry, T. J. Phillips, T. C. Sangster, M. S. Singh, M. A. Stoyer, M. Tabak, G. L. Tietbohl, M. Tsukamoto, K. Wharton, and S. C. Wilks, Phys. Plasmas 5, 1966 (1998).

12. For the case of fast ignition, numerical simulations ${ }^{13}$ indicate that the electron transport is highly filamented due to Weibel instability, ${ }^{15}$ which occurs at early times when beam density $n_{b}$ is comparable to critical density $n_{c}$. Plasma heating can be characterized by collective beam stopping due to coalescence of current filaments and related ion dynamics. Subsequently, however, as these electrons enter the dense plasma region where $n_{e} / n_{b}>>1$, the interaction can be envisioned as the linear superposition of individual, isolated electrons interacting with plasma, which is the focus of this article.

13. M. Honda, J. Meyer-ter-Vehn, and A. Pukhov, Phys. Rev. Lett. 85, 2128 (2000); L. Gremillet, G. Bonnaud, and F. Amiranoff, Phys. Plasmas 9, 941 (2002).

14. M. J. Berger and S. M. Seltzer, in Studies in Penetration of Charged Particles in Matter, Nuclear Science Series, Committee on Nuclear Science, Report 39, Publication 1133 (National Academy of Sciences-National Research Council, Washington, DC, 1964), pp. 205-268; L. Pages et al., At. Data 4, 1 (1972), and references therein. These results are very close to that of the range calculation of this article.

15. E. S. Weibel, Phys. Rev. Lett. 2,83 (1959).

16. H. H. Hubbell, Jr. and R. D. Birkhoff, Phys. Rev. A, 26, 2460 (1982).

17. C. Deutsch et al. have a factor-of-2 error in the electron continuousslowing-down stopping power [Eq. (3) of Ref. 9]; in both their erratum and the original paper, this leads to about a factor-of- 2 overestimate in the electron range [Figs. 1(a) and 2(a) of Ref. 9 and erratum].

18. M. D. Rosen et al., Phys. Rev. A 36, 247 (1987).

19. B. Yaakobi, C. Stoeckl, T. Boehly, D. D. Meyerhofer, and W. Seka, Phys. Plasmas 7, 3714 (2000).

20. N. F. Mott, M. A. Gonville, and C. College, Proc. R. Soc. Lond. A, Math. Phys. Sci. CXXXV, 429 (1932).

21. R. D. Evans, The Atomic Nucleus, International Series in Pure and Applied Physics (McGraw-Hill, New York, 1955).

22. Only the first term has been kept in this differential cross section. This approach is equivalent to the first-order Born approximation (the second term has an order of $\alpha Z, \alpha=1 / 137$, the fine-structure constant). For heavy elements, the $e-i$ cross section will need to be corrected.
23. C. Møller, Ann. Physik (Leipz) 14, 531 (1932).

24. Since small-angle scattering dominates, we have neglected terms that are not associated with this process. Furthermore, this expression is approximately accurate only for $\gamma \leqslant 10$.

25. P. A. Hughes, ed. Beams and Jets in Astrophysics, Cambridge Astrophysics Series (Cambridge University Press, Cambridge, England, 1991).

26. A. Pukhov, Z. M. Sheng, and J. Meyer-ter-Vehn, Phys. Plasmas 6 2847(1999), and references within.

27. H. W. Lewis, Phys. Rev. 78, 526 (1950), and references therein.

28. S. Goudsmit and J. L. Saunderson, Phys. Rev. 57, 24 (1940); ibid. 58, 36; H. A. Bethe, Phys. Rev. 89, 1256 (1953).

29. Since $\sigma_{1}(E)(d E / d s)^{-1} \propto v_{D}(E) / v_{E}(E) E$, where $v_{D}\left(v_{E}\right)$ is the rate of scattering (energy loss), deflection from initial direction is a function of the ratios of these frequencies. Furthermore, since both $\sigma_{1}$ and $d E / d s$ have nearly the same dependence on $\ln \Lambda$, a consequence that is is sufficiently large (i.e., $\sim 5$ ), any sensitive dependence on the Coulomb logarithm nearly cancels out.

30. For plasmas such as discussed herein, the Debye screening length is the standard approximation for the cutoff. 1,2

31. J. D. Jackson, Classical Electrodynamics, 2nd ed. (Wiley, New York, 1975).

32. E. Rohrlich and B. C. Carlson, Phys. Rev. 93, 38 (1954).

33. In Deutsch et al., their penetration is about four times smaller than their range for 1-MeV electrons [Figs. 1(a) and 2(a) of Ref. 9 and erratum]. Even including electron-scattering effects, the results of the present article indicate a penetration that is only a factor of 1.32 smaller than the range of $1-\mathrm{MeV}$ electrons. As best we can tell, the approach of Deutsch et al. is unjustified since they invoke an approximation by P. C. Hemmer and I. E. Farquhar [Phys. Rev. 168, 294 (1968); see Eq. (67) and discussion], which is valid only for small angle deflections and small energy loss. This approximation is invalid in the present context of large deflections and total energy loss.

34. K.-H. Weber, Nucl. Instrum. Methods 25, 261 (1964).

35. Whether considering fast ignition or electron preheat, the distribution of electron energies about their mean will smear out the energy deposition, making it more uniform. 


\section{Operation of a Single-Photon-Counting X-Ray CCD Camera Spectrometer in a Petawatt Environment}

\section{Introduction}

Single-photon-counting x-ray CCD (charge-coupled device) spectrometers are frequently used in ultrashort-pulse laser experiments, mostly for $K$-shell spectroscopy. ${ }^{1-3}$ For singlephoton counting, the incident $x$-ray flux is attenuated such that the probability that two $\mathrm{x}$-ray photons hit a single pixel is small. Consequently, the pixel value of each readout pixel is proportional to the deposited energy from the incident $\mathrm{x}$-ray photon. If the $x$-ray energy is not too high $(<100 \mathrm{keV})$, a significant fraction of the $x$-ray photons deposit all their energy in one pixel. In this case, a histogram of the pixel values provides a good approximation of the incident x-ray spectrum. This technique has the advantage of requiring almost no alignment and the potential of providing absolute $\mathrm{x}$-ray flux information. Consequently single-photon-counting x-ray CCD spectrometers are also used in astronomical satellites, ${ }^{4,5}$ where an extensive set of calibration and characterization data exists. For the satellite data, the low number of incident photons is the biggest issue, whereas in ultrashort-pulse laser experiments, photon counts are generally very high. Signal-to-background issues, especially in a high-energy petawatt environment, become dominant. ${ }^{3}$ Shielding strategies against background $\mathrm{x}$ rays must be carefully chosen to obtain high-quality spectra. In this article, results from a recent experimental campaign at the petawatt facility of the Rutherford Appleton Laboratory (RAL) are presented showing successful strategies to improve the signal-to-background ratio.

\section{Experimental Setup}

The single-photon-counting $\mathrm{x}$-ray spectrometer consists of a Spectral Instruments Series 800 Camera using a 2-k $\times 2-k-$ pixel, back-thinned CCD chip with a pixel size of $13.5 \mu \mathrm{m} .^{6}$ The CCD was cooled to $-35^{\circ} \mathrm{C}$ to reduce the dark current, and the images were recorded with 16-bit resolution.

The camera was mounted $3.8 \mathrm{~m}$ from the target outside the target chamber on a 1-m vacuum tube. Mounting the camera in air and using thin vacuum windows was not possible because the $\mathrm{x}$ rays of interest $-\mathrm{Cu} K$-shell radiation at $\sim 8 \mathrm{keV}-$ are strongly absorbed in air. The RAL petawatt target chamber is very well shielded with $10 \mathrm{~cm}$ of lead on three sides and on top. The side where the access doors are located is unshielded but backed by a curtain shield of $10 \mathrm{~cm}$ of lead and $60 \mathrm{~cm}$ of concrete (Fig. 98.38). The CCD camera was shielded against $\mathrm{x}$ rays scattered from structures close to the target with up to four lead collimators of 10-cm length inside the target chamber and the vacuum tube (inner shielding). The CCD camera housing was surrounded by up to $10 \mathrm{~cm}$ of lead to shield against $\mathrm{x}$ rays from the sides and the back of the CCD (outer shielding). A matched $K$-edge filter was used to attenuate the $K$-shell signal to maintain single-photon counting. Figure 98.39 shows the transmission of the $150 \mu \mathrm{m} \mathrm{Cu}$ filter used for $\mathrm{Cu} K$-shell spectroscopy. Compared to a simple high-pass filter against the thermal radiation from the target, a $K$-edge filter attenuates the spectrum above the lines of interest, thus improving the signalto-background ratio.

The targets were irradiated with 1053-nm pulses from the RAL Vulcan petawatt laser, which delivers up to $\sim 500 \mathrm{~J}$ in $\sim 1$ ps in a 60 -cm-diam beam. ${ }^{7,8}$ These pulses are focused with an $f / 3$ off-axis parabola to a focal spot of $\sim 10-\mu \mathrm{m}$ FWHM. Losses in the compressor and aberrations generally

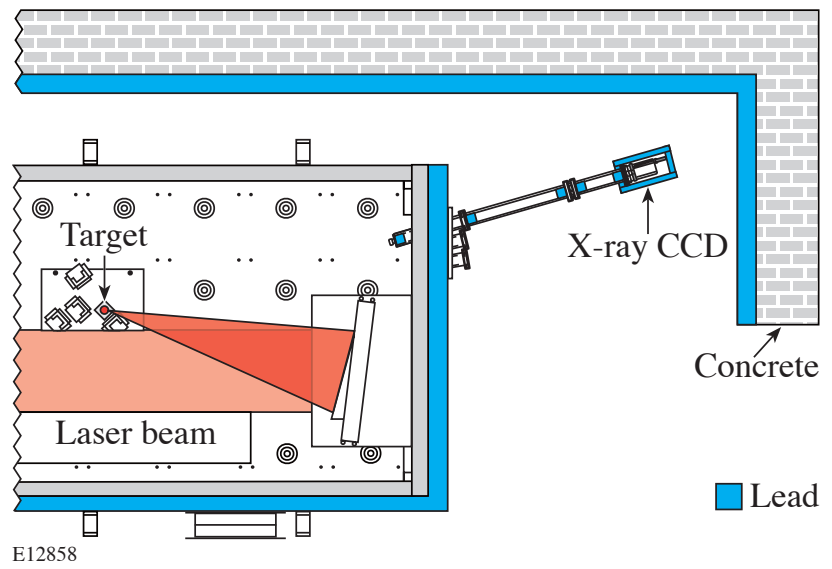

Figure 98.38

Setup of the single-photon-counting X-ray CCD at the RAL petawatt facility, showing the target-area shielding and the CCD shielding. 


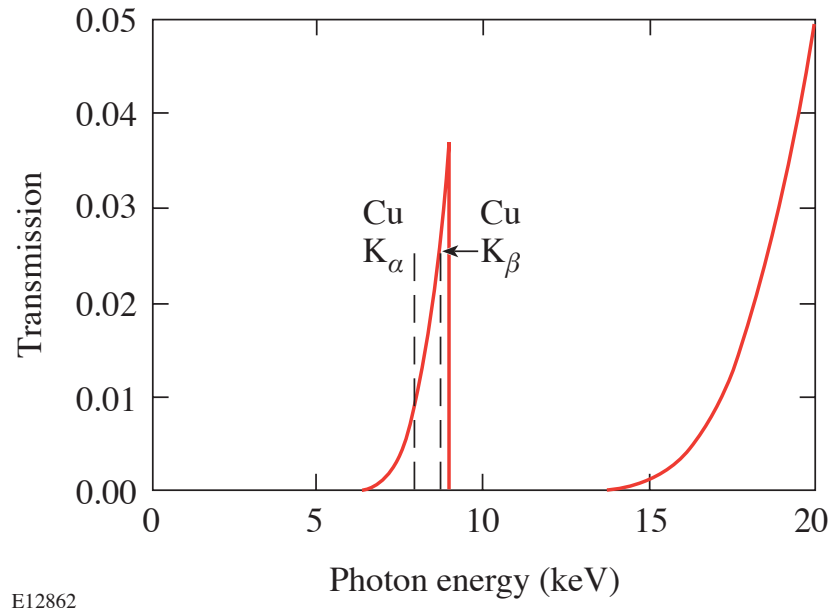

Figure 98.39

A matched $K$-edge filter was used to improve the signal-to-background ratio (150 $\mu \mathrm{m} \mathrm{Cu}$ for $\mathrm{Cu} K$-shell spectroscopy shown). The positions of the $\mathrm{Cu} \mathrm{K}_{\alpha}$ and $\mathrm{K}_{\beta}$ lines are indicated in the graph.

limit the focusable energy to $<50 \%$ of the laser energy. Consequently, the maximum intensity on target was estimated to be $\sim 2 \times 10^{20} \mathrm{~W} / \mathrm{cm}^{2}$.

\section{Optimizing the Shielding}

A series of experiments were conducted to optimize the inner and outer shields and to assess the relative importance of the x-ray background from structures close to the target that reach the CCD from its face and fluorescence and scattered photons that reach the CCD from the side or back.

Figure 98.40(a) shows the histogram from a $20-\mu \mathrm{m}$ thick $\mathrm{Cu}$ target irradiated with an $\sim 250-\mathrm{J}, 1-\mathrm{ps}$ pulse, at $1 \times$ $10^{20} \mathrm{~W} / \mathrm{cm}^{2}$ using 10 -cm inner shielding and no outer shielding. Figure 98.40(b) shows the $K$-shell spectrum after background subtraction. The energy scale is inferred from the published energies of $\mathrm{Cu} \mathrm{K} \alpha=8.05 \mathrm{keV}$ and $\mathrm{Cu} \mathrm{K}_{\beta}=8.90 \mathrm{keV}$. The third line visible in the spectrum is identified as the $\mathrm{He}_{\alpha}$ line of $\mathrm{Cu}$ at $8.36 \mathrm{keV}$.

The high background seen in these experiments distorts the spectrum and makes it almost impossible to discern the $\mathrm{Cu} \mathrm{K}$ line.

Adding $5 \mathrm{~cm}$ of lead as outer shielding around the CCD camera dramatically improves both the background and the quality of the spectrum as shown in Fig. 98.41. This indicates that most of the background is coming from either Comptonscattered primary $\mathrm{x}$ rays or $\mathrm{x}$-ray fluorescence in the structures around the CCD. The spectrum shows the $\mathrm{Cu} \mathrm{K}_{\beta}$ line
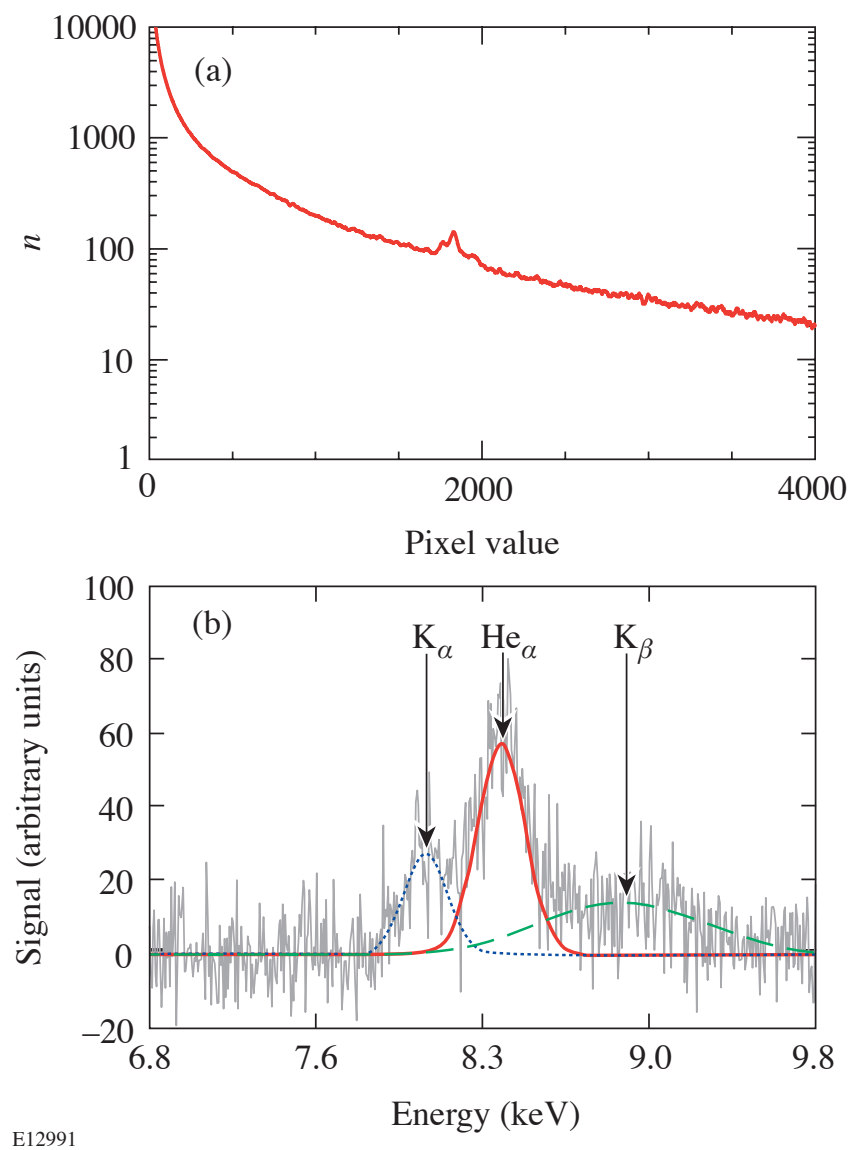

Figure 98.40

CCD histogram (a) and $K$-shell spectrum (b) from a $20-\mu$ m-thick Cu target irradiated with a $\sim 250-\mathrm{J}, 1$-ps laser pulse, at $1 \times 10^{20} \mathrm{~W} / \mathrm{cm}^{2}$ using $10-\mathrm{cm}$ inner shielding and no outer shielding.

clearly separated, and the $\mathrm{Cu} \mathrm{K}_{\alpha}$ and the He-like feature can be easily distinguished.

Increasing the laser energy by a factor of 2 results in a dramatically increased background. Even improving the inner shielding to $40 \mathrm{~cm}$ and the outer shielding to $10 \mathrm{~cm}$ does not prevent the background from rising by about a factor of 3 (see Fig. 98.42). Fortunately in this experiment the x-ray flux also rose by almost a factor of 3 and the spectrum is still well resolved. A new, fourth line is seen in the spectrum, which is identified as $\mathrm{Cu} \mathrm{Ly}{ }_{\alpha}$ at $8.64 \mathrm{keV}$.

\section{Summary and Conclusions}

Efficient shielding is required to obtain high-quality $\mathrm{x}$-ray spectra from a single-photon-counting x-ray CCD spectrometer in a petawatt environment. Shielding the direct line of sight against $\mathrm{x}$ rays from structures close to the target was not sufficient to decrease the background. Only by shielding the 
CCD camera from all sides with $10 \mathrm{~cm}$ of lead was it possible to reduce the background to a level low enough to be able to obtain high-quality spectra even at 500-J laser energy. Scaling the shielding to even higher laser energies or intensities could be difficult given the significant rise in background from 250-J to 500-J energy.

\section{ACKNOWLEDGMENT}

This work was supported by the U.S. Department of Energy Office of Inertial Confinement Fusion under Cooperative Agreement No. DE-FC0392SF19460, the University of Rochester, and the New York State Energy Research and Development Authority. The support of DOE does not constitute an endorsement by DOE of the views expressed in this article.

\section{REFERENCES}

1. F. N. Beg et al., Phys. Plasmas 4, 447 (1997).

2. F. Ewald, H. Schwoerer, and R. Sauerbrey, Europhys. Lett. 60, 710 (2002).
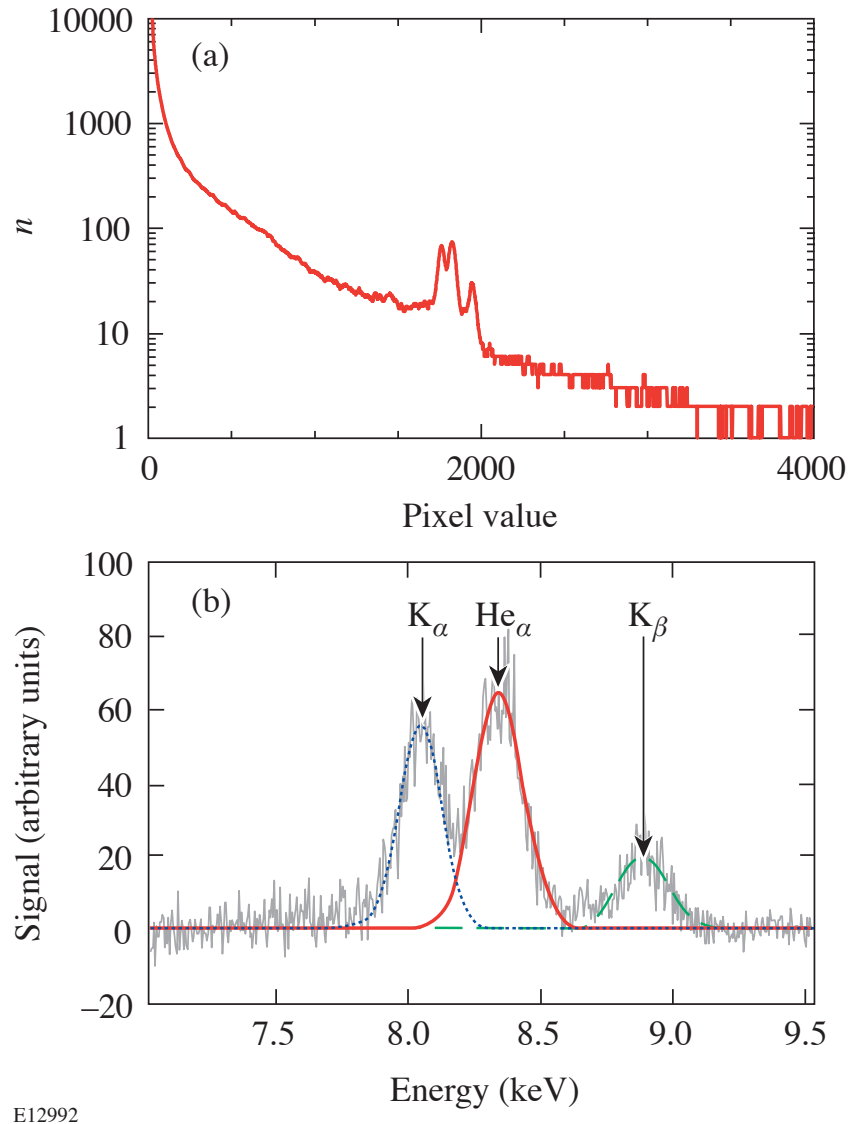

Figure 98.41

CCD histogram (a) and $K$-shell spectrum (b) from a $20-\mu$ m-thick $\mathrm{Cu}$ target irradiated with an $\sim 250-\mathrm{J}, 1$-ps laser pulse, at $1 \times 10^{20} \mathrm{~W} / \mathrm{cm}^{2}$ using $10-\mathrm{cm}$ inner shielding and 5 -cm outer shielding.
3. K. Yasuike et al., Rev. Sci. Instrum. 72, 1236 (2001).

4. M. Nishiuchi et al. in EUV, X-Ray, and Gamma-Ray Instrumentation for Astronomy IX, edited by O. H. Siegmund and M. A. Gummin (SPIE, Bellingham, WA, 1998), Vol. 3445, pp. 268-277.

5. M. Nishiuchi et al., Nucl. Instrum. Methods Phys. Res. 436, 79 (1999).

6. Spectral Instruments, Tucson, AZ 85745 .

7. C. B. Edwards et al., in the 25th International Congress on HighSpeed Photography and Photonics, edited by C. Cavailler, G. P. Haddleton, and M. Hugenschmidt (SPIE, Bellingham, WA, 2003), Vol. 4948, pp. 444-451.

8. C. N. Danson et al., in Inertial Fusion Sciences and Applications 2001, edited by K. A. Tanaka, D. D. Meyerhofer, and J. Meyer-terVehn (Elsevier, Paris, 2002), pp. 479-483.

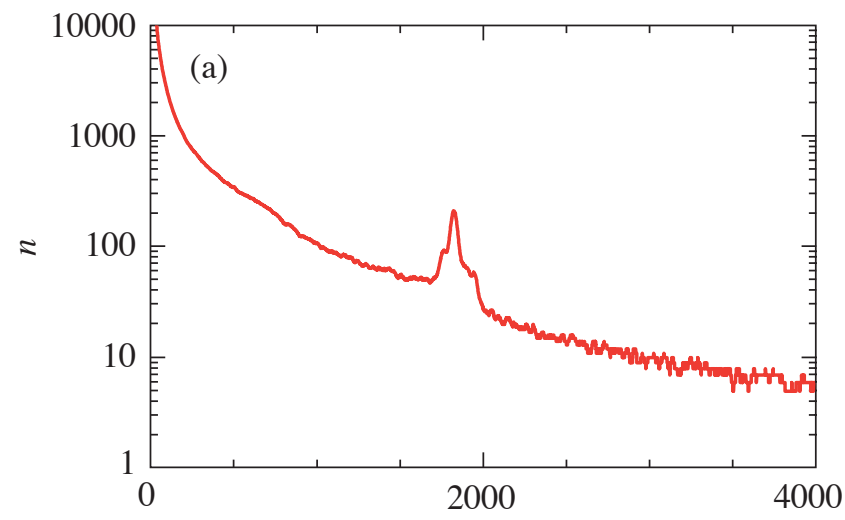

Pixel value

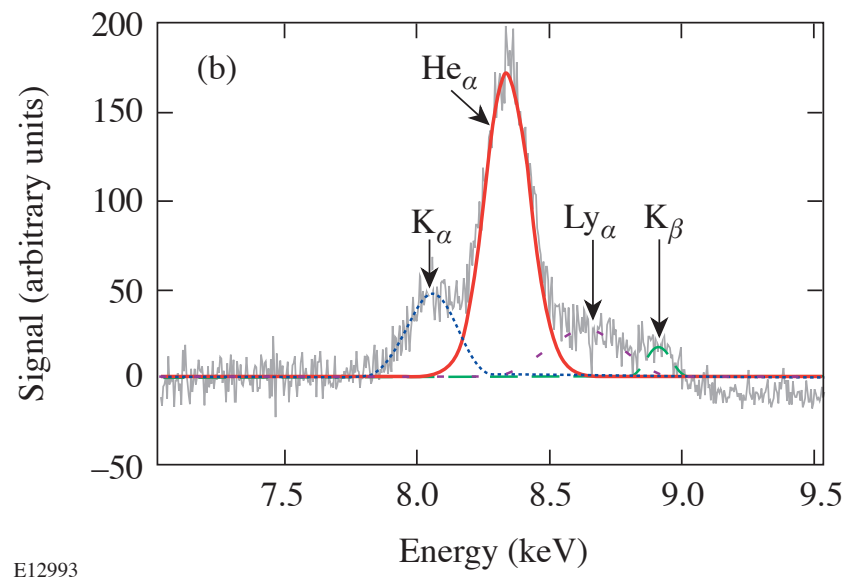

Figure 98.42

CCD histogram (a) and $K$-shell spectrum (b) from a $20-\mu$ m-thick Cu target irradiated with an $\sim 500-\mathrm{J}, 1$-ps laser pulse, at $1 \times 10^{20} \mathrm{~W} / \mathrm{cm}^{2}$ using $40-\mathrm{cm}$ inner shielding and $10-\mathrm{cm}$ outer shielding. 


\section{Theory of Laser-Induced Adiabat Shaping in Inertial Fusion Implosions: The Relaxation Method}

\section{Introduction}

In recent years it has been theoretically shown that the stability of inertial fusion implosions can be significantly improved by shaping the entropy inside the shell. The optimum adiabat shape in the shell consists of a profile that is monotonically decreasing from the outer to the inner surface. Large values of the adiabat on the outer shell surface increase the ablation velocity $V_{a}$, which follows a power law of the outer-surface adiabat $\alpha$ out,

$$
V_{a} \sim \alpha_{\text {out }}^{3 / 5}
$$

while low adiabat values on the inner surface lead to improved ignition conditions and larger burn fraction. A more-detailed history and target design implications of adiabat shaping can be found in the introduction of Ref. 1, which is mostly devoted to the adiabat shape induced by a strong decaying shock. Shaping by a decaying shock ${ }^{1,2}$ requires a very strong prepulse, followed by a low-intensity foot of the main pulse, to launch a strong shock, which decays inside the shell shortly after the prepulse is turned off. The decaying shock (DS) leaves behind a monotonically decreasing adiabat profile, which follows a power law of the mass coordinate

$$
\alpha=\alpha_{\text {in }}\left(\frac{m_{\text {shell }}}{m}\right)^{\Delta_{\mathrm{DS}}} \text {, }
$$

where $m$ is the mass calculated from the outer surface, $m_{\text {shell }}$ is the total shell mass, and $\Delta_{\text {DS }}$ varies between 1.06 and 1.13 depending on the prepulse duration. Two-dimensional simulations $^{2}$ of all-DT, OMEGA-sized capsule implosions have confirmed that DS adiabat targets exhibit significantly reduced Rayleigh-Taylor growth on the ablation surface during the acceleration phase with respect to the flat-adiabat targets. Comparisons between flat- and shaped-adiabat targets are typically carried out by designing the flat- and shaped-adiabat pulses to generate identical adiabats on the inner shell surface.
A different technique aimed at shaping the adiabat is the so-called shaping by relaxation (or RX shaping) described in Ref. 3. The relaxation technique uses a less-energetic prepulse than the DS technique. The RX prepulse is used to launch a shock that may or may not decay inside the shell. In both cases, the prepulse is turned off before the prepulse shock reaches the shell's inner surface. Since the prepulse is followed by a complete power shutoff, the outer portion of the shell expands outward, generating a relaxed density profile, while the prepulse shock travels inside the shell. The prepulse shock is not intended to greatly change the shell adiabat even though it may cause a significant adiabat modification. The main adiabat shaping occurs later in time when the foot of the main pulse starts, driving a strong shock up the relaxed density profile. The main shock first encounters the low-density portion of the relaxed profile, setting it on a very high adiabat. The adiabat develops a monotonically decreasing profile as a result of the increasing pre-shock density. Figure 98.43 shows a plot of the

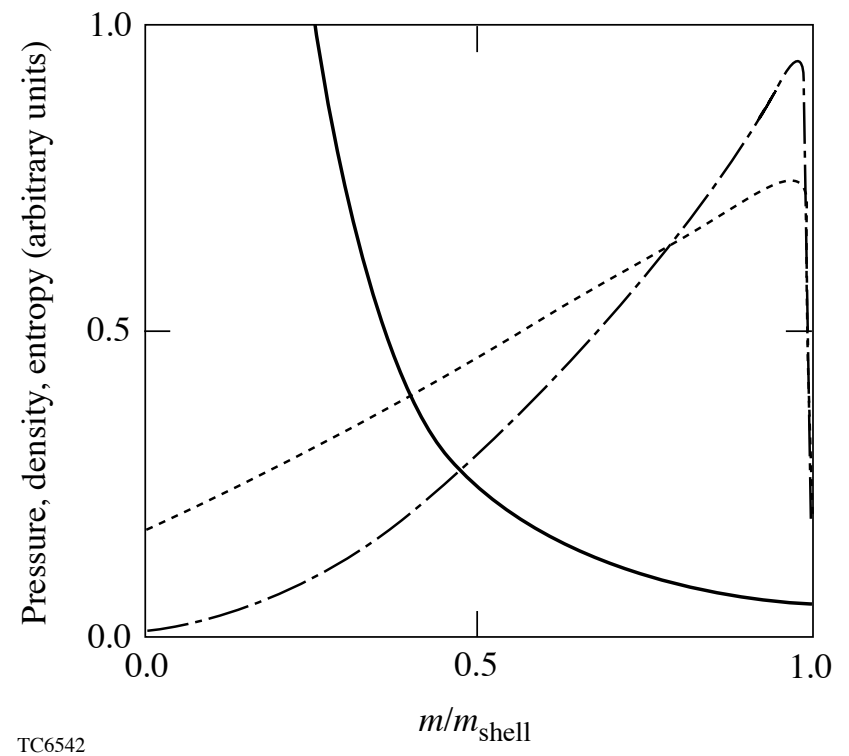

Figure 98.43

Pressure (dashed), density (dot-dashed), and adiabat (solid) profiles generated by a strong shock supported by constant pressure propagating through a relaxed density profile. 
pressure, density, and entropy profiles generated by a strong shock propagating through a relaxed density profile. Observe that the adiabat profile is strongly shaped with large values on the outer shell surface and low values on the inner shell surface. To produce a monotonically decreasing adiabat profile, it is important to design the laser pulse so that the prepulse and main shock merge at the inner shell surface. The RX adiabatshaping technique can be viewed as a two-step process: the prepulse and power shutoff are needed to generate the relaxed density profile, following which the foot of the main pulse shapes the adiabat.

In this article, a detailed hydrodynamic analysis of the relaxed profile generation as well as the shock propagation through these profiles is carried out.

\section{The General Model}

The general gasdynamic model governing the hydrodynamic motion of a compressible gas is greatly simplified by adopting a Lagrangian frame of reference where the independent spatial coordinate is the mass. In planar geometry, the mass coordinate is defined as the mass per unit area calculated from the outer shell surface:

$$
m=\int_{0}^{\bar{x}} \rho\left(\bar{x}^{\prime}, 0\right) d \bar{x}^{\prime}
$$

where $\rho(\bar{x}, 0)$ is the initial density and $\bar{x}$ is the initial spatial location of the fluid elements.

In this coordinate, the outer shell surface is represented by $\bar{x}=0$ and $m=0$. In spherical geometry, Eq. (3) should be replaced by $m=\int_{0}^{\bar{r}} \bar{r}^{\prime 2} \rho\left(\bar{r}^{\prime}, 0\right) d \bar{r}^{\prime}$. For simplicity, we carry out the calculation for the case of an ideal gas with adiabatic index $\gamma$ and neglect convergence effects on the basis that the adiabat shaping occurs when the inner shell surface has not yet moved and the initial aspect ratio is sufficiently large that the shell can be approximated with a uniform slab.

In the Lagrangian frame and away from the shock front, the planar equations of motion for the shocked material can be written in the following conservative form:

$$
\begin{gathered}
\frac{\partial u}{\partial m}-\frac{\partial}{\partial t} \frac{1}{\rho}=0 \\
\frac{\partial u}{\partial t}+\frac{\partial P}{\partial m}=0
\end{gathered}
$$

$$
p=S(m) \rho^{\gamma}
$$

where $u, P, \rho$, and $S(m)$ are the velocity, pressure, density, and entropy, respectively. The function $S(m)$ is referred to as the adiabat and depends exclusively on the Lagrangian coordinate. At the shock front, the physical quantities must satisfy the Hugoniot conditions, which in the strong shock regime can be written in the following simple form:

$$
\begin{gathered}
{\left[\rho_{\mathrm{ps}}\right]_{m_{\mathrm{sh}}}=\frac{\gamma+1}{\gamma-1}\left[\rho_{0}\right]_{m_{\mathrm{sh}}},} \\
{\left[u_{\mathrm{ps}}\right]_{m_{\mathrm{sh}}}=\frac{2}{\gamma-1} \frac{\dot{m}_{\mathrm{sh}}}{\left.\rho_{\mathrm{ps}}\right]_{m_{\mathrm{sh}}}}} \\
\dot{m}_{\mathrm{sh}}=\sqrt{\frac{(\gamma-1)}{2}\left[P_{\mathrm{ps}} \rho_{\mathrm{ps}}\right]_{m_{\mathrm{sh}}}}
\end{gathered}
$$

where $P_{\mathrm{ps}}$ represents the post-shock pressure, $\rho_{\mathrm{ps}}$ is the postshock density, $u_{\mathrm{ps}}$ is the post-shock velocity, and $\rho_{0}$ is the initial unshocked density. Here, $m_{\mathrm{sh}}$ is the mass coordinate corresponding to the shock location. More details on the Lagrangian model used here can be found in Ref. 1 .

\section{The Generation of the Relaxed Profiles}

When a square laser prepulse precedes the main laser pulse, a pressure pulse is applied to the shell's outer surface, launching a uniform shock followed by a rarefaction wave, which causes a relaxation of the pressure and density profiles. Since the leading edge of the rarefaction wave travels faster than the shock, it eventually catches the shock unless the shock reaches the inner shell surface before interacting with the rarefaction wave. Two different relaxed profiles are generated depending on whether or not the rarefaction leading edge catches the prepulse shock inside the shell. If the shock and rarefaction do not merge in the shell, the resulting relaxed profiles are said to be of the "first kind" while merging leads to relaxed profiles of the "second kind."

It is convenient to define with subscript $p$ the prepulse quantities, $P_{p}, \rho_{p}, S_{p}, a_{p}$, and $u_{p}$, representing the induced pressure, compressed density, adiabat, sound speed, and flow velocity while the uniform laser prepulse of duration $\Delta t_{p}$ is applied. Using the prepulse quantities, we define the following set of dimensionless quantities and coordinates: 


$$
\begin{gathered}
\hat{\rho} \equiv \frac{\rho}{\rho_{p}}, \quad \hat{P} \equiv \frac{P}{P_{p}}, \quad \hat{u} \equiv \frac{u}{a_{p}}, \\
\hat{S}=\frac{S}{S_{p}}, \quad z=\frac{m}{m_{*}}, \quad \tau=\frac{t}{\Delta t_{*}},
\end{gathered}
$$

where

$$
\begin{gathered}
\Delta t_{*}=\frac{\Delta t_{p}}{\sqrt{2 \gamma /(\gamma-1)}-1}, \\
m_{*}=\Delta t_{*} \rho_{p} a_{p}=\Delta t_{*} \sqrt{\gamma P_{p} \rho_{p}} .
\end{gathered}
$$

Here $\Delta t *$ is the travel time of the rarefaction wave before catching the shock, and $m_{*}$ is the areal density undertaken by the rarefaction wave's leading edge before catching the prepulse shock. Furthermore, $\tau=0$ is defined as the time when the laser prepulse is terminated and the rarefaction wave is launched. It follows that $\tau=1$ represents the shock-rarefaction interaction time.

These dimensionless variables can be used to rewrite the Lagrangian equations of motion in the convenient form

$$
\begin{gathered}
\frac{\partial \hat{u}}{\partial z}=\frac{\partial \hat{\rho}^{-1}}{\partial \tau}, \\
\gamma \frac{\partial \hat{u}}{\partial \tau}=-\frac{\partial \hat{P}}{\partial z} \\
\hat{P}=\hat{S}(z) \hat{\rho}^{\gamma}
\end{gathered}
$$

Observe that Eqs. (14)-(16) can be combined into the following single equation:

$$
\gamma \frac{\partial^{2}}{\partial \tau^{2}} \frac{1}{\hat{\rho}}+\frac{\partial^{2}}{\partial z^{2}} \hat{S}(z) \hat{\rho}^{\gamma}=0
$$

which can be solved for $\hat{\rho}$ once $\hat{S}$ is known. There is no general solution of the equations of motion after the rarefaction wave is launched; however, one can consider two limiting cases resulting in two different relaxed profiles. The first is the case when the rarefaction wave catches the shock at the shell's rear surface. This case is characterized by values of $m_{*}=m_{\text {shell }}$. The second is the case when the rarefaction wave quickly catches the shock near the outer surface, causing the shock to decay throughout most of the shell. This case requires a small $m_{*}$ satisfying $m_{*}<m_{\text {shell }}$. We will consider these two cases separately.

1. Relaxed Profiles of the First Kind: Rarefaction and

Shock Merge at the Rear Surface of the Shell: $m_{*}=m_{\text {shell }}$

In this case, the relaxed density and pressure profiles are the ones generated by the rarefaction wave, whose functional forms can be obtained from Ref. 1 or from most textbooks on compressible flow. In the mass coordinate, the density profile can be written in the following simple form:

$$
\rho(z<\tau)=\rho_{p}\left(\frac{z}{\tau}\right)^{\frac{2}{\gamma+1}}=\rho_{p}\left(\frac{m}{m_{\mathrm{rf}}}\right)^{\frac{2}{\gamma+1}}
$$

where $m_{\mathrm{rf}}=a_{p} \rho_{p} t$ represents the trajectory of the rarefaction wave's leading edge. For $z>\tau$, the density is uniform and equal to the post-shock density $\rho=\rho_{p}$.

2. Relaxed Profiles of the Second Kind: Rarefaction

Catches the Shock Inside the Shell: $m_{*}<m_{\text {shell }}$

In this case, the derivation of the relaxed profiles is significantly more complicated since there is no exact solution of the equations of motion after the rarefaction wave catches the prepulse shock. After the rarefaction wave reaches the shock at $z=1$, the latter decays, leaving behind a relaxed profile with two distinct spatial shapes in the regions $0<m<m_{*}$ and $m_{*}<m<m_{s}^{p}$, where $m_{s}^{p}$ is the location of the prepulse shock. In the dimensionless variable $z=m / m_{*}$, those two regions are $0<z<1$ and $1<z<z_{s}^{p}$, where $z_{s}^{p} \equiv m_{s}^{p} / m_{*}$.

a. The region $z<1$. One could speculate that the profiles in the region $0<z<1$ have a similar shape to the ones generated by the rarefaction wave, $\rho \sim z^{2 / \gamma+1}$; however, this assumption does not take into account a second profile relaxation occurring when a sound wave travels backward down the rarefactionwave profile right after the rarefaction wave catches the shock. The solution in the regions $0<z<1$ must satisfy Eq. (17) and the boundary conditions at $z=0$ and $z=1$. At $z=0$, the vacuum boundary condition requires that $\rho(z=0)=0$. At $z=1$, both the pressure and its gradient must be continuous to prevent separation of the continuous medium requiring that 


$$
\begin{aligned}
& P\left(z=1^{-}, \tau\right)=P\left(z=1^{+}, \tau\right), \\
& \frac{d P}{d z}\left(z=1^{-}, \tau\right)=\frac{d P}{d z}\left(z=1^{+}, \tau\right) .
\end{aligned}
$$

It would be unrealistic to hope that a simple power law in $z$ would exactly satisfy the equations of motion and the boundary conditions; however, one could attempt to look for an approximate solution behaving as a power law of the mass coordinate

$$
\hat{\rho} \approx \bar{\rho}(\tau) z^{\alpha}
$$

which, by construction, satisfies the boundary conditions at $z=0$. Substituting Eq. (20) into Eq. (17) with $\hat{S}=1$ leads to the following two conditions:

$$
\begin{gathered}
\frac{d^{2}}{d \tau^{2}} \frac{1}{\bar{\rho}}+\alpha(\alpha \gamma-1) \bar{\rho}^{\gamma} \approx 0, \\
F_{1}(\alpha) \equiv \frac{\alpha(\gamma+1)}{2} \approx 1 .
\end{gathered}
$$

Note that the symbol $\approx$ has been used to indicate that the function (20) is meant to represent an approximate rather than an exact solution. To solve Eq. (21), one needs two initial conditions for $\bar{\rho}$ and $d \bar{\rho} / d \tau$ at $\tau=1^{+}$just after the rarefaction wave interacts with the shock. While the initial condition for the density at $z=1$ is trivial,

$$
\bar{\rho}\left(\tau=1^{+}\right)=1
$$

the condition on the time derivate at $\tau=1^{+}$is rather complicated. The exact derivation of $\hat{\rho}^{\prime}(1,1)$ is described in Ref. 1 and leads to Eq. (56) of Ref. 1, which reads as

$$
\left[\frac{d \bar{\rho}}{d \tau}\right]_{\tau=1^{+}}=-\frac{6 /(\gamma+1)}{\frac{3}{2}+\sqrt{\frac{\gamma}{2(\gamma-1)}}+\sqrt{\frac{\gamma-1}{2 \gamma}}} .
$$

It is worth mentioning that the initial condition on the density spatial profile at $\tau=1$ requires that the function $\hat{\rho}(\tau=1, z) \approx \bar{\rho}(1) z^{\alpha}$ reproduces the rarefaction-wave solution $\hat{\rho}(1, z)=z^{2 / \gamma+1}$, thus requiring that the power index $\alpha$ satisfies $\alpha-2 /(\gamma+1) \approx 0$. This condition is identical to Eq. (22) and does not represent an additional condition. Note that a simple analytical solution of Eq. (21) is the following power law:

$$
\bar{\rho}(\tau)=\left[\frac{2(\gamma-1)}{\alpha(\gamma+1)^{2}(\alpha \gamma-1)}\right]^{\frac{1}{\gamma+1}} \frac{1}{\frac{2}{\tau^{\gamma+1}}} .
$$

Equation (25) satisfies the initial conditions (23) and (24) only if the following conditions are met:

$$
\begin{gathered}
F_{2}(\alpha) \equiv\left[\frac{2(\gamma-1)}{\alpha(\gamma+1)^{2}(\alpha \gamma-1)}\right]^{\frac{1}{\gamma+1}} \approx 1, \\
F_{3}(\alpha) \equiv \frac{1}{3}\left[\frac{2(\gamma-1)}{\alpha(\gamma+1)^{2}(\alpha \gamma-1)}\right]^{\frac{1}{\gamma+1}} \\
\times\left(\frac{3}{2}+\sqrt{\frac{\gamma}{2(\gamma-1)}}+\sqrt{\frac{\gamma-1}{2 \gamma}}\right) \approx 1 .
\end{gathered}
$$

To test Eq. (20) against the remaining boundary conditions (19) at $z=1$, one needs to determine the solution for $z>1$ carried out in the next section. In any case, the condition (22) implies that $\alpha \approx 2 /(\gamma+1)$, indicating that the density profile shape is little changed by the second relaxation occurring after the shock-rarefaction merging.

b. The region $1<z<z_{s-}^{p}$. As mentioned earlier, the shock decays for $z>1, \tau>1$. The entropy profile left behind by the decaying shock is calculated in Ref. 1 and approximately follows a power law of the Lagrangian coordinate $m$ (or $z$ ):

$$
\hat{S}(z) \simeq \frac{1}{z^{\delta}},
$$

where $\delta \simeq 1.31$ when the effects of ablation are neglected and the spatial range is limited to $z<10$. Typically, the range $1<z<10$ includes most (if not all) of the ICF RX target 
designs for both OMEGA-like as well as NIF-like capsules. However, if $z>10$, the shock decay becomes self-similar and the power-law index asymptotically approaches the value $\delta \simeq$ 1.275. The residual ablation pressure leads to a somewhat slower decay and can be accounted for through a lower $\delta$ (approximately 17\% lower) as indicated in Ref. 1 . In the selfsimilar solution, the density is a function of the coordinate

$$
\xi=\frac{z}{z_{s}^{p}}
$$

where $z_{s}^{p}$ is the trajectory of the decaying shock, satisfying the Hugoniot condition

$$
\dot{z}_{s}^{p}=\sqrt{\frac{\gamma-1}{2 \gamma} \frac{1}{\left(z_{s}^{p}\right)^{\delta}}} .
$$

Equation (30) can be easily integrated with the initial condition $z_{s}^{p}(1)=1$, leading to the following form of the decelerating shock trajectory:

$$
z_{s}^{p}(\tau)=\left[1+\left(1+\frac{\delta}{2}\right) \sqrt{\frac{\gamma-1}{2 \gamma}}(\tau-1)\right]^{\frac{2}{2+\delta}}
$$

The density $\hat{\rho}(\xi)$ does not follow a power law. Instead it must satisfy a complicated second-order differential equation derived by substituting Eqs. (16) and (28) into (17), leading to

$$
\xi \frac{d}{d \xi}\left[1+\frac{\delta}{2}+\xi \frac{d}{d \xi}\right] \frac{1}{\hat{\rho}}+\frac{2}{\gamma-1} \frac{d^{2}}{d \xi^{2}} \frac{\hat{\rho}^{\gamma}}{\xi^{\delta}}=0
$$

Equation (32) cannot be exactly integrated; however, one can again attempt to look for an approximate solution in the form of a power law of the self-similar coordinate

$$
\hat{\rho} \approx \xi^{\mu}
$$

which approximately satisfies not only Eq. (32) but also the boundary by conditions at $z=1$ [Eqs. (19)] and at the shock front $z=z_{s}^{p}$. In order for the simple power law (33) to approximate the solution of Eq. (32), the power index $\mu$ must satisfy the following conditions obtained upon substitution of (33) into (32):

$$
\begin{gathered}
G_{1}(\mu) \equiv \frac{\mu(\gamma+1)}{\delta+2} \approx 1, \\
G_{2}(\mu) \equiv \frac{\mu(\gamma-1)\left(1+\frac{\delta}{2}-\mu\right)}{2(\mu \gamma-\delta)(\mu \gamma-\delta-1)} \approx 1 .
\end{gathered}
$$

The boundary conditions at the shock front are provided by the Hugoniot conditions that determine $\hat{\rho}$ and $d \hat{\rho} / d \xi$ at $\xi=1$. Note that the density gradient can be obtained from Eqs. (35b) and (36) of Ref. 1 for $\xi=1$, yielding the following condition:

$$
\frac{d \hat{\rho}}{d \xi}(\xi=1)=\frac{3 \delta}{\gamma+1}
$$

While the Hugoniot condition on the density $\hat{\rho}(1)=1$ is trivially satisfied by $\hat{\rho}=\xi^{\mu}$, the condition on $d \hat{\rho} / d \xi$ requires that

$$
G_{3}(\mu) \equiv \frac{\mu(\gamma+1)}{3 \delta} \approx 1
$$

The next step is to verify that Eq. (33) used for $z>1$ satisfies Eqs. (19) at $z=1$. Since the entropy is continuous at $z=1$, the first of Eq. (19) requires that the density be continuous; therefore $\hat{\rho}\left(z=1^{-}, \tau\right) \approx \hat{\rho}\left(z=1^{+}, \tau\right)$, yielding

$$
\bar{\rho}(\tau) \approx \frac{1}{\left[z_{s}^{p}(\tau)\right]^{\mu}}
$$

Then, using the continuity of the density and Eqs. (28) and (33) into the second of Eq. (19), one finds the condition

$$
H(\alpha, \mu) \equiv \frac{\gamma \alpha+\delta}{\gamma \mu} \approx 1
$$

The last step is to find the two power indices $\alpha$ and $\mu$ in such a way that all the conditions [Eqs. (22), (26), (27), (34), (35), (37), and (39)] are met and that Eq. (38) is approximately satisfied for any time $\tau$ limited by $z_{s}^{p}(\tau) \leq 10$ representing the range of interest for ICF capsule design. 
c. Determination of the power indices. The power indices $\alpha$ and $\tau$ can be determined by plotting the conditions

$$
\begin{gathered}
G_{1}(\mu) \approx 1, \quad G_{2}(\mu) \approx 1, \quad G_{3}(\mu) \approx 1, \\
F_{1}(\alpha) \approx 1, \quad F_{2}(\alpha) \approx 1, \quad F_{3}(\alpha) \approx 1, \\
H(\alpha, \mu) \approx 1,
\end{gathered}
$$

and determining $\alpha$ and $\mu$ so that all such conditions are approximately satisfied. Figure 98.44 shows the plot of three functions $G_{1}, G_{2}$, and $G_{3}$ that depends exclusively on the power index $\mu$ for a given $\gamma=5 / 3$ and $\delta \simeq 1.315$. Observe that all the $G$-functions exhibit a zero near $\mu \simeq 1.45$, which can be considered as an approximate solution of all the Eqs. (40). After determining $\mu$, the $F$-functions are plotted together with the function $H$ versus the parameter $\alpha$ (Fig. 98.45), clearly indicating that $\alpha \simeq 0.75$ is an approximate solution of all Eqs. (41) and (42). The last step is to verify that Eq. (38) is approximately satisfied for any time $\tau$ and for $z_{s}^{p} \leq 10$. First, it is easily found from Eq. (31) that the condition $z_{s}^{p} \leq 10$ requires $\tau \leq 61$. Second, we plot both sides of Eq. (38) versus time for $\tau \leq 61$ (Fig. 98.46) and realize that both functions are approximately equal over the range of interesting times.

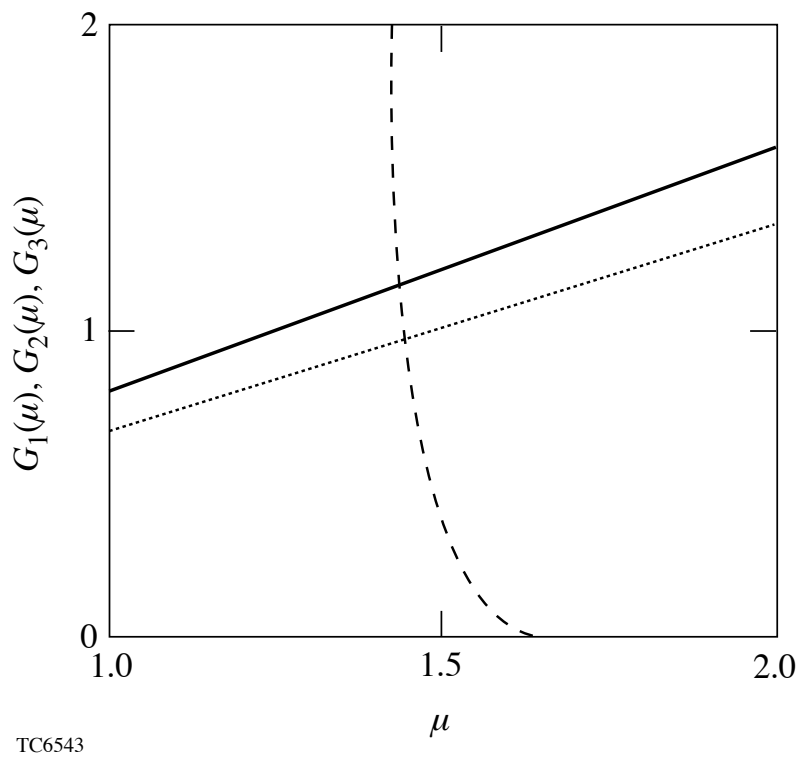

Figure 98.44

The functions $G_{1}(\mu)$ (solid), $G_{2}(\mu)$ (dashed), and $G_{3}(\mu)$ (dotted) are all approximately equal to 1 at $\mu=1.45$.

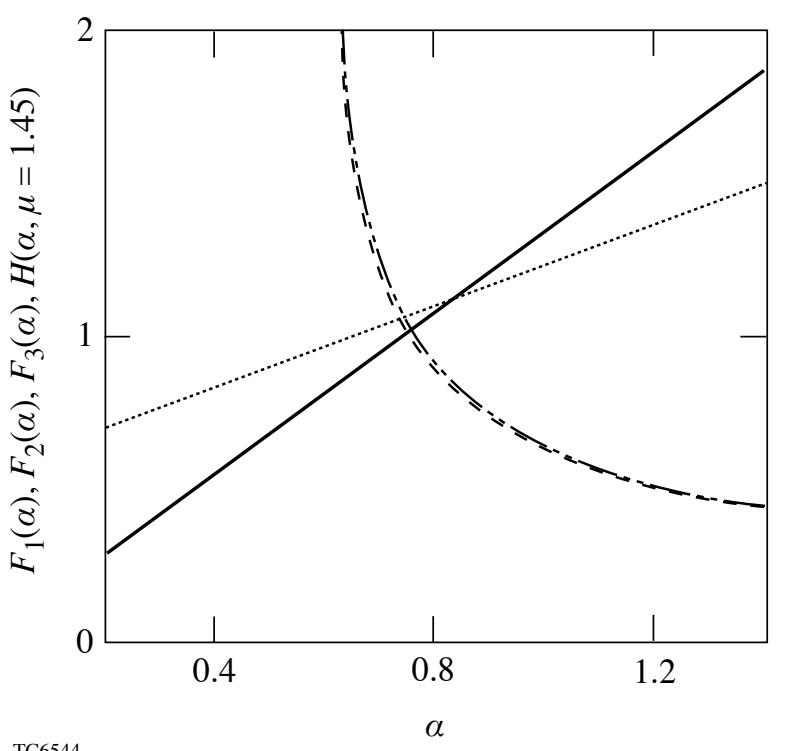

Figure 98.45

The functions $F_{1}(\alpha)$ (solid), $F_{2}(\alpha)$ (dashed), $F_{3}(\alpha)$ (dashed-double-dotted), and $H(\alpha, \mu=1.45)$ (dotted) are all approximately equal to 1 at $\alpha=0.75$.

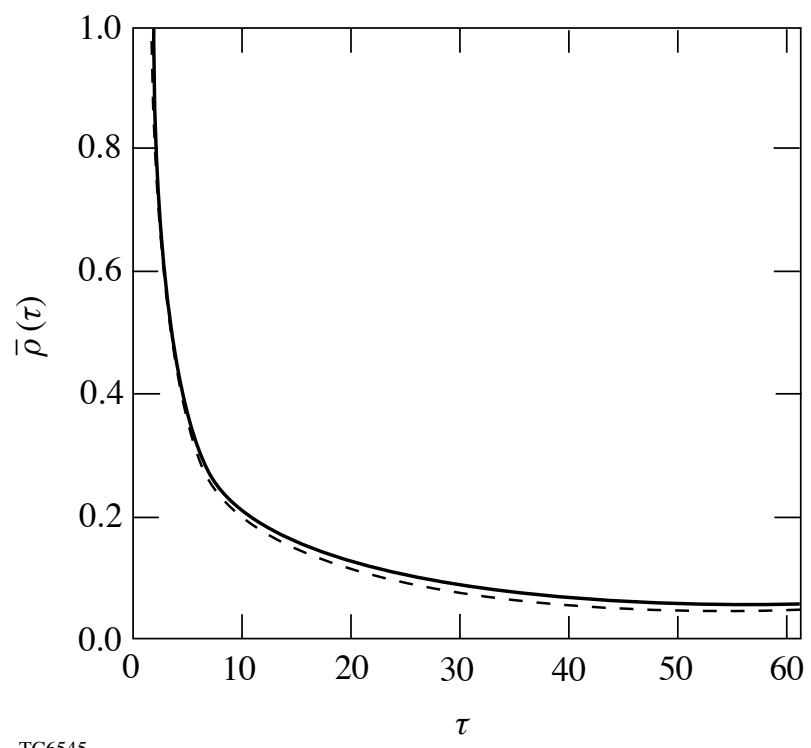

Figure 98.46

The analytic solution of Eqs. (21), (23), and (24) for $\bar{\rho}(\tau)$ given by Eq. (25) (solid) is compared to the approximate solution for $\bar{\rho}(\tau)$ given by Eq. (38) using $\mu=1.45$ (dashed). The plot indicates good agreement between the two functional forms of $\bar{\rho}(\tau)$. 
In summary, all the equations of motions and boundary conditions for $\tau>1$ are approximately satisfied by simple power laws of the Lagrangian coordinate $z$, leading to the following solutions for $\gamma=5 / 3$ :

$$
\begin{gathered}
\rho(z<1) \approx \rho_{p}\left(\frac{z}{\tau}\right)^{3 / 4}=\rho_{p}\left(\frac{m}{m_{\mathrm{rf}}}\right)^{3 / 4}, \\
\rho\left(1<z<z_{s}^{p}\right) \approx \rho_{p}\left(\frac{z}{z_{s}^{p}(\tau)}\right)^{1.45}=\rho_{p}\left(\frac{m}{m_{s}^{p}(\tau)}\right)^{1.45},
\end{gathered}
$$

where $m_{s}^{p}$ represents the trajectory of the prepulse shock $m_{s}^{p}=z_{s}^{p} m_{*}$ and $m_{\mathrm{rf}}=a_{p} \rho_{p} t$. Here $t=0$ represents the end of the prepulse. This concludes the calculation of the relaxed profiles. The next step is to determine the adiabat shape induced by the main shock propagating through the relaxed profiles of the first and second kind described by Eqs. (18) and [(43), (44)], respectively.

\section{Main Shock Propagation Through the Relaxed Profiles}

Before the prepulse shock reaches the rear surface, the main shock is launched by the foot of the main laser pulse and supported by the applied pressure $P_{f}$, which is assumed constant during the main shock propagation. The adiabat shaping occurs when the main shock travels up the relaxed density profile, shocking material with increasing density to lower and lower adiabats. The main shock is typically a strong shock in the sense that the ratio of pressures across the shock front is much larger than unity. Using the strong-shock form of the Hugoniot relations leads to a great simplification of the hydrodynamic analysis and is often accurate for the main shock propagation. However, it is important to emphasize that some of the strong-shock Hugoniot relations are accurately satisfied only for a very large pressure jump. For instance, the density jump across a strong shock with $Z=\left(P_{2}-P_{1}\right) / P_{1} \gg 1$ is

$$
\frac{\rho_{2}}{\rho_{1}}=\frac{\gamma+1}{\gamma-1}-\frac{4 \gamma}{(\gamma-1)^{2}} \frac{1}{Z}+O\left(\frac{1}{Z^{2}}\right)
$$

Observe that even for large $Z$, the first-order corrections can be significant due to the large coefficient $-4 /(\gamma-1)^{2}$. For instance, in a gas with $\gamma=5 / 3$, this coefficient is -15 and the leading order term is 4 , thus indicating that the $1 / Z$ correction is small only when $Z>>15 / 4$. Another implication of a strong main shock is the fact that the shocked material evolves on the time scale of its own sound speed, which scales as $\sqrt{P_{M}}$, where $P_{M}$ is the main shock pressure. Instead, the relaxed profiles evolve on a slower time scale of the order of the prepulse shock sound speed, which scales as $\sim \sqrt{P_{p}}$. It follows that in the limit of $\sqrt{P_{M} / P_{p}}>>1$, one can neglect the dynamics of the relaxed profiles during the main shock propagation. In other words, one can regard the relaxed profiles as frozen in time while the main shock propagates through. Obviously, the corrections due to a finite $\sqrt{P_{M} / P_{p}}$ may be large and need to be estimated.

For the sake of simplicity, we will first proceed by neglecting the finite main shock strength correction, assume that the relaxed profiles are frozen, and determine the lowest-order solution. The finite shock strength effects will be estimated later as corrections to the lowest-order solution.

Effects of mass ablation and residual ablation pressures, though important, are also neglected in this article, and the calculation focuses on the ideal case of a strong shock supported by a constant applied pressure traveling up a relaxed density profile described by the power laws (18) or [(43), (44)].

\section{Shock Propagation Through a Relaxed Profile of the First Kind}

\section{As indicated earlier in Relaxed Profiles of the First Kind} (p. 108), the density profile generated by a rarefaction wave before its interaction with the prepulse shock is described by a simple power law of the areal density $\rho=\left(\mathrm{m} / \mathrm{m}_{\mathrm{rf}}\right)^{\alpha}$ with $\alpha=2 /(\gamma+1)=0.75$. Here $m_{\mathrm{rf}}=a_{p} \rho_{p} t$ is the location of the rarefaction leading edge. If the main shock is much stronger than the prepulse shock, then the relaxed profile may be considered as frozen in time during the fast main shock propagation. Since both shocks must merge at the shell's inner surface, the fast main shock is launched when the prepulse shock is approaching the inner shell surface. One can therefore approximate $m_{\mathrm{rf}} \approx m_{\text {shell }}$ to lowest order in the inverse shock strength.

a. The approximation of a static relaxed profile. Introducing the new variable $\zeta \equiv m / m_{\mathrm{rf}} \approx m / m_{\text {shell }}$, the relaxed profile can be represented by the simple power law

$$
\rho=\rho_{p} \xi^{\alpha}, \quad \alpha=\frac{2}{\gamma+1},
$$

with $0<\zeta<1$. To simplify the analysis, we introduce another dimensionless variable related to the main shock location: 


$$
\xi=\frac{m}{m_{s}^{M}}=\frac{\zeta}{\xi_{s}^{M}}
$$

where both $m_{s}^{M}$ and $\xi_{s}^{M}$ represent the main shock location in their respective coordinates. Obviously, $0<\xi<1$ with $\xi=1$ representing the shock position. The profiles left behind by the main shock can be written in terms of the variables $\xi$ and $\xi$ in the following form:

$$
\begin{gathered}
\rho=\frac{\rho_{M} \xi^{\alpha}}{\Phi(\xi)^{1 / \gamma}}, \quad S=\frac{S_{M}}{\xi^{\gamma \alpha}}, \\
P=\frac{P_{M}}{\Phi(\xi)}, \quad u=a_{M} \frac{U(\xi)}{\xi^{\alpha / 2}},
\end{gathered}
$$

where

$$
\begin{gathered}
\rho_{M} \equiv \rho_{p}(\gamma+1) /(\gamma-1), \\
P_{M}=S_{M} \rho_{M}^{\gamma}, \\
a_{M} \equiv \sqrt{\gamma P_{M} / \rho_{M}},
\end{gathered}
$$

and $\Phi(\xi), U(\xi)$, and $S_{M}$ need to be determined. By inspection of Eqs. (48) and (49), it is clear that by setting $\Phi(1)=1$, the pressure immediately behind the shock is $P_{M}$. Furthermore, using the Hugoniot condition for the post-shock velocity, one can immediately deduce that $U(1)=\sqrt{2 / \gamma(\gamma-1)}$. Substituting Eqs. (48) and (49) into the equations of motion and using the Hugoniot condition for the shock velocity,

$$
\dot{m}_{S}^{M}=\sqrt{\frac{\gamma-1}{2} p_{M} \rho_{M}\left(\zeta_{S}^{M}\right)^{\alpha}}
$$

it is straightforward to derive the two equations governing $\Phi$ and $U$ :

$$
\xi \sqrt{\frac{\gamma-1}{2 \gamma}} \frac{d \Phi^{1 / \gamma}}{d \xi}-\frac{\alpha}{2} \frac{U}{\xi^{1-(\alpha / 2)}}+\xi^{\alpha / 2} \frac{d U}{d \xi}=0
$$

$$
\sqrt{\frac{\gamma(\gamma-1)}{2}} \xi^{1-(\alpha / 2)} \frac{d U}{d \xi}-\frac{d}{d \xi} \frac{1}{\Phi}=0
$$

which need to be solved with the initial conditions $\Phi(1)=1$ and $U(1)=\sqrt{2 / \gamma(\gamma-1)}$. The unknown $S_{M}$ can be determined by requiring that the solution of Eqs. (51) and (52) reproduces the applied pressure $P_{f}$ at $m=0$. Using Eq. (49), one finds the following expression for $S_{M}$ and the pressure behind the shock $P_{M}$ :

$$
S_{M}=\frac{P_{f} \Phi(0)}{\rho_{M}^{\gamma}}, \quad P_{M}=P_{f} \Phi(0)
$$

Observe that the pressure behind the shock is constant throughout the shock propagation. An approximate yet quite accurate solution of Eqs. (51) and (52) can be found by using the following ansatz:

$$
\Phi(\xi) \approx \frac{\Phi(0)}{1+\xi[\Phi(0)-1]}
$$

Substituting Eq. (54) into (52) and using the boundary condition for the velocity at the shock front yields

$$
U(\xi) \approx \sqrt{\frac{2}{\gamma(\gamma-1)}}\left\{\left[1-\frac{1}{\Phi(0)}\right] \frac{2}{\alpha}\left(\xi^{\alpha / 2}-1\right)+1\right\} .
$$

The functions (54) and (55) must approximately satisfy Eq. (51), which can be rewritten upon substitution of the two functions as

$$
\left[2-\alpha-\frac{2}{\Phi(0)}\right] \frac{1}{\xi^{1-(\alpha / 2)}} \approx \frac{\gamma-1}{\gamma} \frac{\Phi(0)-1}{\Phi(0)} \xi \Phi(\xi)^{(\gamma+1) / \gamma} .
$$

Observe that since $\alpha<2$, the left-hand side of Eq. (56) is singular for $\xi \rightarrow 0$ while the right-hand side is regular. It follows that Eq. (56) can be satisfied only when $2-\alpha-2 / \Phi(0) \approx 0$, leading to

$$
\Phi(0) \approx \frac{2}{2-\alpha}=\frac{\gamma+1}{\gamma}=1.6 .
$$


The numerical solution of Eqs. (51) and (52) yields $\Phi(0)$ $\simeq 1.68 \mathrm{in} \mathrm{good}$ agreement with the analytic derivation. Figure 98.47 compares the numerically derived functions $\bar{p} \equiv \Phi(0) / \Phi(\xi)$ (representing the pressure profile) and $\bar{u} \equiv U(\xi) / \xi^{\alpha / 2}$ (representing the velocity profile) with the analytic solutions from Eqs. (54), (57), and (55). Observe that the pressure increases approximately linearly before the shock, while the velocity profile is approximately flat. The shock pressure is amplified about 1.6 to 1.7 times with respect to the applied pressure. This amplification is due to the slowing down of the shocked material against the shock front. The shock-front velocity decreases in time as a power law. It can be easily derived by first determining the shock trajectory in the mass coordinate through Eq. (50) and then substituting the post-shock velocity $u_{\mathrm{ps}}$ calculated at the shock front [Eq. (49) at $\xi=1$ and $\left.m=m_{s}^{M}\right]$ into the shock relation
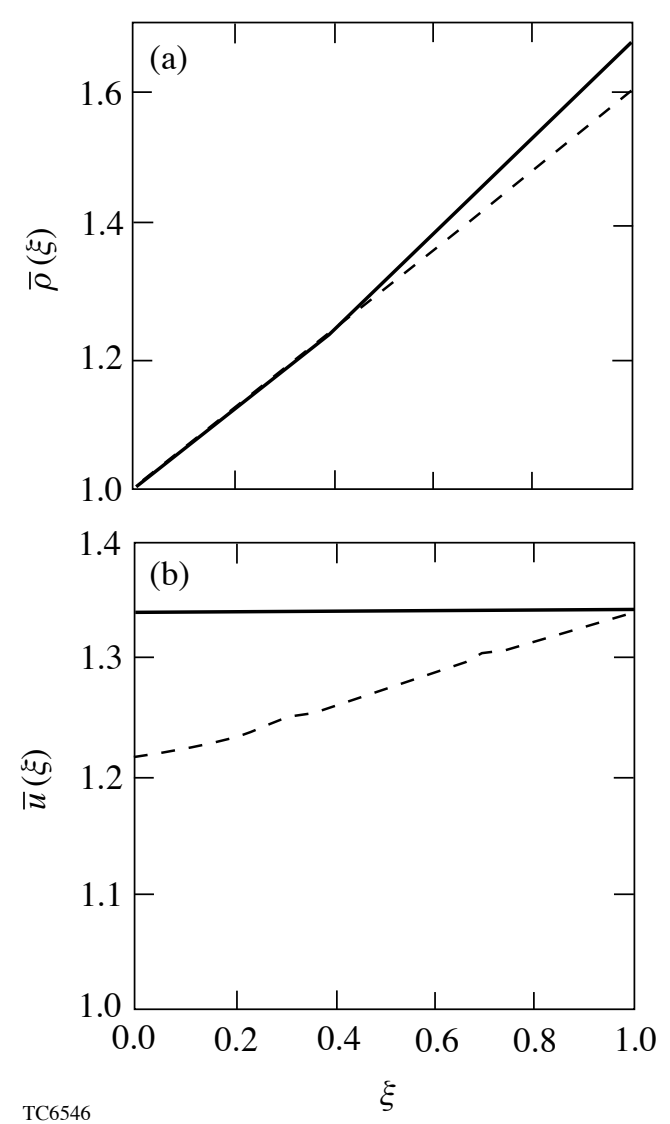

Figure 98.47

The numerical (solid) and analytic (dashed) solutions of the self-similar (a) pressure profiles $\bar{p}(\xi)$ and (b) density profile $\bar{\rho}(\xi)$ are shown to be in relatively good agreement.

$$
U_{\text {shock }}=[(\gamma+1) / 2] u_{\mathrm{ps}} \text {. }
$$

This leads to the following expression for the shock velocity:

$$
U_{\text {shock }}=\frac{a_{M} \sqrt{\frac{(\gamma+1)^{2}}{2 \gamma(\gamma-1)}}}{\left[\sqrt{\frac{\gamma-1}{2 \gamma}} \frac{2-\alpha}{2} \frac{\rho_{M} a_{M} t}{m_{\text {shell }}}\right]^{\alpha /(2-\alpha)}},
$$

which decreases as $1 / t^{0.6}$ for $\alpha=2 /(\gamma+1)=0.75$.

Furthermore, as indicated by Eqs. (48) and (53), the entropy profile behind the main shock follows a simple power law of the mass coordinate

$$
S(m)=\frac{P_{f} \Phi(0)}{\rho_{M}^{\gamma}}\left(\frac{m_{\text {shell }}}{m}\right)^{\gamma \alpha},
$$

where the rarefaction leading edge $m_{\mathrm{rf}}$ has been taken near the inner surface at $m_{\mathrm{rf}}=m_{\text {shell }}$ and $\alpha=2 /(\gamma+1)$. Indeed, it is important to require that the main shock, the rarefaction leading edge, and the prepulse shock merge at the target's rear surface, leading to $m_{\mathrm{rf}} \simeq m_{\text {shell }} \simeq m_{*}$. This timing requirement is discussed in the Introduction (p. 106), where the optimized adiabat shaping procedure is described. Figure 98.48 shows the simulated adiabat profile generated by a strong shock driven by a 26-Mbar applied pressure traveling up a relaxed target with a density profile represented by the following power law:

$$
\rho=\rho_{p}\left[\frac{(1-\alpha) \rho_{p} x}{m_{\text {shell }}}\right]^{\frac{\alpha}{1-\alpha}},
$$

where $0<x<m_{\text {shell }} /(1-\alpha) \rho_{p}$ is the spatial coordinate. Observe that the profile in Eq. (60) requires that $\alpha<1$ to avoid singularities at $x=0$ (here $\alpha=0.75$ ). Note Eq. (60) can be easily rewritten in terms of the mass coordinate $m$ leading to $\rho=\rho_{p}\left(m / m_{\text {shell }}\right)^{\alpha}$, which is identical to Eq. (18) with $m_{\mathrm{rf}}$ $=m_{\text {shell. }}$. The solid curve of Fig. 98.48 represents the adiabat from the 1-D simulation, while the dashed curve is obtained from the analytic theory in Eq. (59). Observe that the two adiabat profiles are virtually identical, indicating excellent agreement between theory and simulations. 


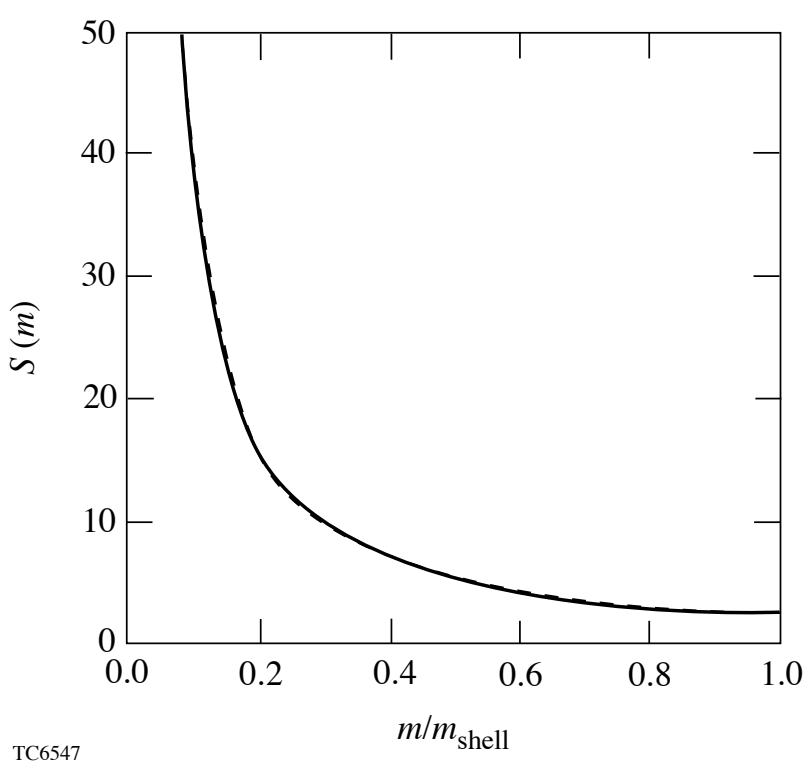

Figure 98.48

A comparison of the simulated (solid) and analytically calculated [Eq. (59)] (dashed) adiabat shapes generated by propagating a shock supported by a constant 26-Mbar pressure through a density profile of the first kind shows excellent agreement.

b. Corrections due to dynamic relaxed profiles/finite shock strength. The analytic theory above has been derived in the limit of a relaxed profile that is stationary during the shock propagation. However, the profile varies in time as the leading edge of the rarefaction wave travels toward the shell's inner surface. Because of these changes, the resulting adiabat profile left behind by the main shock is somewhat shallower than the one predicted by Eq. (59). One can estimate the effects of dynamic profiles on the adiabat shape by assuming that the relaxed profile changes slightly over the main shock propagation interval. The dynamic corrections to the entropy profiles can be estimated by determining the entropy at the shock front located at $m_{s}^{M}(t)$ :

$$
S\left(m_{s}^{M}\right)=\frac{P_{\mathrm{ps}}^{M}}{\left(\rho_{\mathrm{ps}}^{M}\right)^{\gamma}}
$$

where $P_{\mathrm{ps}}^{M}$ and $\rho_{\mathrm{ps}}^{M}$ are the post-shock pressure and density at the main shock front, respectively. It is obvious that if one can rewrite the right-hand side of Eq. (61) as a function of the shock position, then the entropy profile is given by the RHS with $m_{s}^{M}$ replaced by $m$. The post-shock density in Eq. (61) can be rewritten in terms of the pre-shock density at the shock front $\rho_{\mathrm{bs}}^{M}$ including the linear corrections in the inverse shock strength:

$$
\rho_{\mathrm{ps}}^{M} \simeq \frac{\gamma+1}{\gamma-1} \rho_{\mathrm{bs}}^{M}\left[1-\frac{4 \gamma}{\gamma^{2}-1} \frac{P_{p}}{P_{M}}\left(\frac{m_{s}^{M}}{m_{\mathrm{shell}}}\right)^{\gamma \alpha}\right],
$$

where the post-shock pressure used to calculate the correction of order $P_{p} / P_{M}$ has been taken equal to the zerothorder solution $P_{M}$. Because of the large numerical coefficient $4 \gamma /\left(\gamma^{2}-1\right)$, the first-order correction in the inverse shock strength shown in Eq. (62) needs to be retained. Another important correction is in the pre-shock density $\rho_{\mathrm{bs}}=\rho_{p}\left(\mathrm{~m} / m_{\mathrm{rf}}\right)^{\alpha}$, which varies in time due to the evolution of the rarefaction leading edge $m_{\mathrm{rf}}$, which can be written as

$$
m_{\mathrm{rf}}=m_{\mathrm{shell}}-a_{p} \rho_{p}\left(\Delta t_{s}^{M}+t_{f}-t\right)
$$

where $\Delta t_{s}^{M}$ is the travel time of the main shock through the shell and $t_{f}$ is the beginning time of the laser foot when the main shock is launched. Observe that the second term on the righthand side represents the correction to $m_{\mathrm{rf}}$ and is small as long as the main shock is strong. This can be quantified by calculating $\Delta t_{s}^{M}$ after integrating Eq. (50) and setting $m_{\mathrm{rf}} \simeq m_{\text {shell }}$ into the variable $\zeta$. A straightforward calculation yields

$$
\Delta t_{s}^{M} \simeq \sqrt{\frac{2 \gamma}{\gamma-1}} \frac{2}{2-\alpha} \frac{m_{\text {shell }}}{\rho_{M} a_{M}}
$$

and $\left(t-t_{f}\right) / \Delta t_{s}^{M}=\left(m_{s}^{M} / m_{\text {shell }}\right)^{1-\alpha / 2}$. Substituting Eq. (64) into (63) leads to the following equation for the rarefaction leading edge in terms of the main shock location:

$$
m_{\mathrm{rf}}=m_{\text {shell }}\left\{1-\frac{2}{2-\alpha} \sqrt{\frac{2 \gamma}{\gamma+1} \frac{P_{p}}{P_{M}}}\left[1-\left(\frac{m_{s}^{M}}{m_{\text {shell }}}\right)^{\frac{2-\alpha}{2}}\right]\right\} \text {. }
$$

Observe that the correction to $m_{\mathrm{rf}}$ is of the order of $\sqrt{P_{p} / P_{M}}$ as indicated in the introduction of Main Shock Propagation Through the Relaxed Profiles (p. 112) and can be significant even for a strong main shock. It follows that the post-shock density used in Eq. (61) can be rewritten, including the relevant corrections, in the following form: 


$$
\rho_{\mathrm{ps}}^{M}=\rho_{M}\left(\frac{m_{S}^{M}}{m_{\text {shell }}}\right)^{\alpha}
$$

$$
\times \frac{1-\frac{4 \gamma}{\gamma^{2}-1} \frac{P_{p}}{P_{M}}\left(\frac{m_{s}^{M}}{m_{\text {shell }}}\right)^{\gamma \alpha}}{\left\{1-\frac{2}{2-\alpha} \sqrt{\frac{2 \gamma}{\gamma+1} \frac{P_{p}}{P_{M}}}\left[1-\left(\frac{m_{s}^{M}}{m_{\text {shell }}}\right)^{\frac{2-\alpha}{2}}\right]\right\}^{\alpha}} .
$$

The next step is the calculation of the dynamic correction to the post-shock pressure in Eq. (62). While the exact calculation of such corrections can be cumbersome, a fairly good approximation can be obtained by assuming that the post-main-shock pressure profile is linear in the mass coordinate:

$$
P \approx P_{f}\left[1+A(t) \frac{m}{m_{s}^{M}(t)}\right]
$$

where $A(t)$ needs to be determined. It is important to notice that in the static relaxed profile case, $A(t)$ was previously determined to be approximately constant with $A(t) \approx A_{0} \approx$ $\alpha /(2-\alpha)$ It follows that the dynamic corrections will lead to a change in $A(t)$, which can be rewritten as a small correction to $A_{0}$ such as

$$
A(t)=A_{0}+\delta A(t)+\cdots
$$

where $\delta A \ll A_{0}$ needs to be determined. An important dynamic correction enters the Hugoniot relations for the velocity jump across the main shock

$$
u_{\mathrm{ps}}^{M}=u_{\mathrm{bs}}^{M}+\sqrt{\frac{2}{\gamma+1} \frac{P_{\mathrm{ps}}^{M}}{\rho_{\mathrm{bs}}^{M}}}
$$

where $u_{\mathrm{bs}}^{M}$ is the pre-shock velocity at the shock front given by the standard rarefaction-wave solution

$$
u_{\mathrm{bs}}^{M}=a_{p}\left[\frac{\alpha}{1-\alpha}\left(\frac{m_{s}^{M}}{m_{\mathrm{rf}}}\right)^{1-\alpha}+\sqrt{\frac{2}{\gamma(\gamma-1)}}-\frac{2}{\gamma-1}\right]
$$

Observe that $u_{\mathrm{bs}}$ represents a correction of the order of $\sqrt{P_{p} / P_{M}}$ in the Hugoniot relation [Eq. (69)] and can be evaluated using the lowest-order form of $m_{\mathrm{rf}} \simeq m_{\text {shell }}$. Instead, the pre-shock density $\rho_{\mathrm{bs}}^{M}=\rho_{b}\left(m_{s}^{M} / m_{\mathrm{rf}}\right)^{\alpha}$ in Eq. (69) needs to include the lowest-order corrections for $m_{\text {rf }}$ given in Eq. (65). Conservation of momentum requires that the acceleration balances the pressure gradient at the shock front leading to

$$
\frac{\partial u_{\mathrm{ps}}^{M}}{\partial t}=-\left(\frac{\partial P}{\partial m}\right)_{m_{s}^{M}}
$$

Substituting Eqs. (67)-(70) into (71) yields the following ordinary differential equation for $\delta A$ :

$$
\begin{aligned}
\frac{d}{d \eta}\left(\eta^{2-\alpha} \delta A\right)= & -\frac{2 \alpha}{2-\alpha} \sqrt{\frac{P_{b}}{P_{M}}} \\
& \times\left[\sqrt{\frac{2 \gamma}{\gamma+1}}+\sqrt{2 \gamma(\gamma+1)}\right] \eta^{2-\frac{3 \alpha}{2}},
\end{aligned}
$$

where $\eta \equiv m_{s}^{M} / m_{\text {shell }}$. Equation (72) can be integrated using the initial condition that $\delta A\left(m_{s}^{M}=0\right)=0$, leading to the following final form of the post-shock pressure at the shock front:

$$
\begin{aligned}
P_{\mathrm{ps}}^{M}= & P_{M}\left\{1-\frac{2 \alpha}{3(2-\alpha)} \sqrt{\frac{P_{p}}{P_{M}}}\right. \\
& \left.\times\left[\sqrt{\frac{2 \gamma}{\gamma+1}+\sqrt{2 \gamma(\gamma+1)}}\right] \eta^{1-\frac{\alpha}{2}}\right\} .
\end{aligned}
$$

Observe that the corrections due to finite main shock strength cause the main shock pressure to decrease as the shock propagates through the dynamic relaxed profile. This clearly leads to a gentler decrease in entropy.

The last step is to substitute Eqs. (66) and (73) into (61) and derive the final form of the adiabat shape: 


$$
S(m)=S_{\text {in }}\left(\frac{m_{\text {shell }}}{m}\right)^{\frac{2 \gamma}{\gamma+1}} \frac{\chi\left(\frac{m}{m_{\text {shell }}}\right)}{\chi(1)},
$$

where

$$
S_{\text {in }}=c_{0} \frac{(\gamma-1)^{2 \gamma} \chi(1)}{\gamma(\gamma+1)^{2 \gamma-1}} \frac{P_{f}}{\rho_{0}^{\gamma}}
$$

is the entropy on the inner shell surface, $c_{0}=1.68 / 1.6=1.05$ is a corrective factor to account for the analytical approximation used in Eq. (54), and $\rho_{0}$ is the initial shell density.

The function $\chi(x)$ represents the corrections due to the finite main shock strength

$$
\begin{aligned}
\chi(x)= & {\left[1-\frac{2 \sqrt{2}}{3} \frac{\gamma+2}{\gamma+1} \sqrt{\frac{P_{p}}{P_{f}}} x^{\gamma+1}\right] } \\
& \times \frac{\left[1-\sqrt{\frac{2 P_{p}}{P_{f}}}\left(1-x^{\frac{\gamma}{\gamma+1}}\right)\right]^{\frac{2 \gamma}{\gamma+1}}}{\left[1-\frac{4 \gamma^{2}}{(\gamma+1)^{2}(\gamma-1)} \frac{P_{p}}{P_{f}} x^{\frac{2 \gamma}{\gamma+1}}\right]^{\gamma}},
\end{aligned}
$$

where the relation $\alpha=2 /(\gamma+1)$ has been used and $P_{p} / P_{f}$ is the ratio of the prepulse pressure to the pressure of the foot of the main pulse. This concludes the analysis of the main shock propagation through a relaxed profile of the first kind. The next step is to investigate the main shock propagation through relaxed profiles of the second kind and determine the resulting adiabat shape.

\section{Shock Propagation Through a Relaxed Profile} of the Second Kind

In the case of a short prepulse, the rarefaction wave catches the shock at $m=m_{*}$ before the inner surface $\left(m<m_{\text {shell }}\right)$, and the shock decays until it reaches the inner surface at $m=m_{\text {shell }}$.
As described in Relaxed Profiles of the Second Kind (p. 108), the relaxed profile for $\gamma=5 / 3$ is well-approximated by two power laws of the mass coordinate: $\rho \sim m^{0.75}$ for $m<m_{*}$ and $\rho \sim m^{1.45}$ for $m_{*}<m<m_{\text {shell. }}$. The analysis of the main shock propagation through such a profile is vastly more complicated with respect to the case of the single power-law profile discussed in Shock Propagation Through a Relaxed Profile of the First Kind (p. 112). An approximate analytic solution can be found, however, by assuming that the pressure profile behind the main shock is linear in the mass coordinate. Similar to the case of profiles of the first kind, we will first consider the approximation of infinite main shock strength and static relaxed profiles. The corrections due to the finite shock strength and dynamic profiles are estimated a posteriori as small perturbations of the zeroth-order solution.

a. The approximation of a strong shock and a static relaxed profile. In the static case, the relaxed profile in the region $m$ $<m_{*}$ is identical to the profile of the first kind, leading to a pressure profile behind the shock that linearly increases about $60 \%$ with respect to the applied foot pressure $P_{f}$. Once the main shock enters into the second region $m_{*}<m<m_{\text {shell }}$, an exact analytic solution cannot be found. A careful analysis of the numerical simulation indicates, however, that the pressure profile behind the shock remains approximately linear in the mass coordinate. In contrast with the behavior in the first region, however, the shock-front pressure is not constant while the pressure at $m_{*}$ varies slightly around the value $\omega P_{f}$ with $\omega \simeq 1.5$ to 1.6 for $\gamma=5 / 3$. Thus, it makes sense to look for a solution of the hydrodynamic equation with a linear pressure profile of the following form:

$$
P_{\mathrm{ps}}\left(m>m_{*}\right) \approx \omega(\gamma) P_{\text {foot }}\left[1+D(t)\left(\frac{m}{m_{*}}-1\right)\right] \text {, }
$$

where $\omega(\gamma)$ is a constant that must be chosen to reproduce the pressure at the time when the main shock reaches $m_{*}$. Using the results of the previous section for the main shock propagation for $m<m_{*}$, one can conclude that

$$
\omega(\gamma) \approx \frac{\gamma+1}{\gamma} .
$$

Similar to the analysis in Shock Propagation Through a Relaxed Profile of the First Kind (p. 112), Eq. (77) can be substituted into the momentum equation at the shock front $\left[\partial_{t} u_{\mathrm{ps}}=-\left(\partial_{m} P\right)\right]_{m_{s}^{M}}$, where the post-shock velocity at the 
shock front is given by the Hugoniot relation

$$
u_{\mathrm{ps}}^{M}=\sqrt{2 P_{\mathrm{ps}}^{M} /(\gamma+1) \rho_{\mathrm{bs}}^{\mathrm{M}}}
$$

with the pre-shock density at the shock front given by

$$
\rho_{\mathrm{bs}}^{M}=\rho_{p}\left(\frac{m_{s}^{M}}{m_{\mathrm{shell}}}\right)^{\mu}
$$

where the prepulse shock location $m_{\mathrm{sh}}^{p}$ has been assumed to have reached the inner shell surface so that $m_{s}^{p} \simeq m_{\text {shell }}$. As in Shock Propagation Through a Relaxed Profile of the First Kind (p. 112), $u_{\mathrm{ps}}^{M}$ and $P_{\mathrm{ps}}^{M}$ represent the post-shock velocity and pressure at the main shock front. The resulting shock-front momentum equation can be simplified by using the main shock trajectory $m_{s}^{M}(t)$ as the time coordinate and by using the shock mass velocity $\dot{m}_{s}^{M}=\sqrt{(\gamma+1) P_{\mathrm{ps}}^{M} \rho_{\mathrm{bs}}^{M} / 2}$. A straightforward manipulation of the momentum equation leads to the following simple differential equation for $D$ :

$$
\frac{d D}{d z_{s}^{M}}\left(z_{s}^{M}-1\right)+3 D-\frac{\mu}{z_{s}^{M}}\left[1+D\left(z_{s}^{M}-1\right)\right]=0
$$

where $D=D\left[z_{s}^{M}\right]$ and $z_{s}^{M} \equiv m_{s}^{M}(t) / m_{*}$. It is important to note that the only nonsingular solution of Eq. (80) has the simple form

$$
D(x)=\frac{2\left(x^{\mu}-1\right)+\mu(x-3)(x-1)-\mu^{2}(x-1)^{2}}{(\mu-2)(\mu-1)(x-1)^{3}} .
$$

The pressure at the shock fronts can be determined from Eq. (77) upon substitution of Eq. (81), leading to

$$
P_{\mathrm{ps}}^{M}\left(m_{s}^{M}\right) \approx \omega(\gamma) P_{f}\left[1+D\left(\frac{m_{s}^{M}}{m_{*}}\right)\left(\frac{m_{s}^{M}}{m_{*}}-1\right)\right],
$$

representing a growing function of $m_{s}^{M}$ reaching the asymptotic value of 3.6 for $m_{s}^{M}>m_{*}$. The entropy behind the shock can be easily calculated by substituting the pressure and density at the shock front into the definition of the entropy:

$$
S\left(m_{s}^{M}\right)=\frac{P_{\mathrm{ps}}^{M}\left(m_{s}^{M}\right)}{\left[\rho_{\mathrm{ps}}^{M}\left(m_{s}^{M}\right)\right]^{\gamma}} .
$$

Using Eqs. (77), (81), and (82) into (83) leads to the following form of the entropy:

$$
\begin{aligned}
& S_{\infty}\left(m_{*}<m \leq m_{\text {shell }}\right)=\frac{\omega(\gamma) P_{f}}{\rho_{M}^{\gamma}} \\
& \times\left(\frac{m_{\text {shell }}}{m}\right)^{\gamma \mu}\left[1+D\left(\frac{m}{m_{*}}\right)\left(\frac{m}{m_{*}}-1\right)\right],
\end{aligned}
$$

where $\rho_{M}=\rho_{p}(\gamma+1) /(\gamma-1)$ and the subscript $\infty$ indicates that Eq. (84) is valid only for infinite main shock strength. Figure 98.49 shows a comparison of the predicted adiabat profile of Eq. (84) (dashed) with the simulated adiabat profile (solid) generated by a strong shock driven by a 26-Mbar applied pressure traveling up a static, relaxed target with a density profile given by $\rho=\rho_{M}\left(m_{*} / m_{\text {shell }}\right)^{\mu}\left(m / m_{*}\right)^{\alpha}$ for $m \leq m_{*}$, and $\rho=\rho_{M}\left(m / m_{\text {shell }}\right)^{\mu}$ for $m_{*}<m \leq m_{\text {shell }}$. Here, $m_{*} / m_{\text {shell }}$ is chosen to be 0.05 . The theory again shows excellent agreement with the simulation.

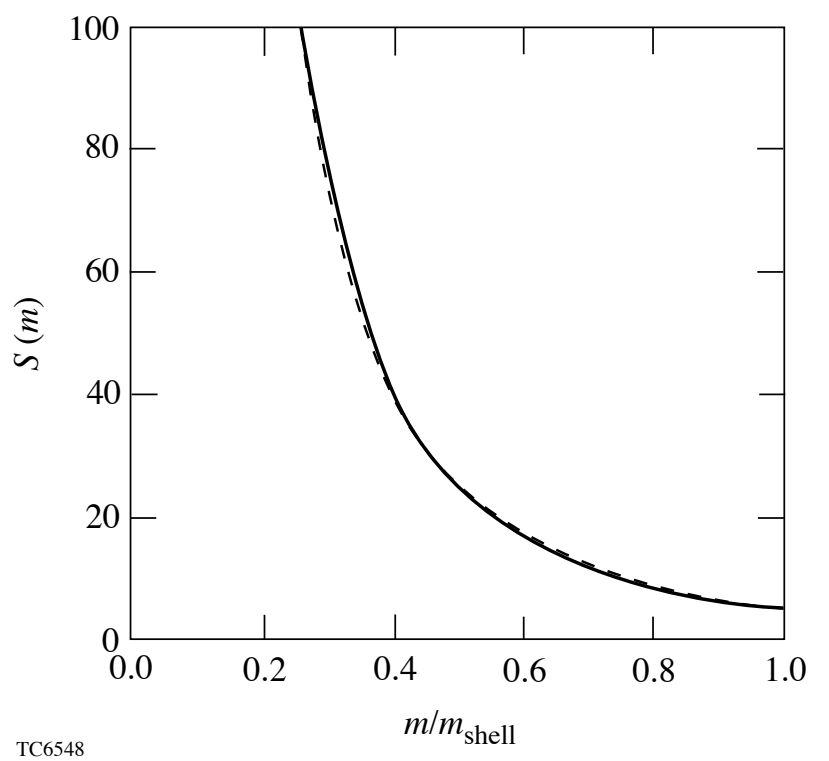

Figure 98.49

A comparison of the simulated (solid) and analytically calculated [Eq. (84)] (dashed) adiabat shape generated by propagating a shock supported by a constant 26-Mbar pressure through a density profile of the second kind shows excellent agreement. 
It is interesting to observe that the linear approximation for the pressure profile leads to a flat velocity profile as shown by substituting Eq. (77) into the momentum equation, yielding

$$
u(m, t)=u_{\mathrm{ps}}^{M}(t) .
$$

The density behind the shock can be determined from the pressure and the entropy through the relation $\rho=(p / S)^{1 / \gamma}$. Upon substitution of the density, the mass conservation equation [Eq. (4)] can be solved for the velocity profile, leading to

$$
u(m, t)=u_{\mathrm{ps}}^{M}\left[1-G\left(z, z_{s}^{M}\right)\right]
$$

where $z=m / m_{*}$ and

$$
\begin{aligned}
G\left(z, z_{s}^{M}\right)= & \frac{\gamma-1}{2 \gamma} \frac{d D\left(z_{s}^{M}\right)}{d z_{s}^{M}} \\
& \left.\times \int_{z_{s}^{M}\left[1+D\left(z_{s}^{M}\right)(x-1)\right]^{(\gamma+1) / \gamma}}^{z} \frac{(x-1)[1+D(x)(x-1)]^{1 / \gamma}}{\left[z_{s}^{M}\right.}\right)^{\mu} d x .
\end{aligned}
$$

Observe that the two velocity profiles obtained from the momentum and mass conservation equations are approximately equal in magnitude as long as $|G|<<1$. The value $G$ can be estimated after replacing $z$ with $\eta z_{s}^{M}$ and by plotting $G\left(\eta z_{s}^{M}, z_{s}^{M}\right)$ for $1 / z_{s}^{M}<\eta<1$ for different values of $z_{s}^{M}$. Figure 98.50 shows the value of $G$ for $z_{s}^{M}=2,5,10,20$, indicating that $G$ does not exceed 0.21 for typical values of $z_{s}^{M} \leq 20$. It follows that the linear pressure profile of Eq. (77), the flat velocity profile of Eq. (85), and the entropy profile of Eq. (84) are accurate approximations of the solution to the hydro equations.

b. Corrections due to dynamic relaxed profiles/finite shock strength. To determine the correction to the adiabat shape due to the finite shock strength and the dynamic evolution of the relaxed profile during the main shock propagation, one needs to calculate the main shock position in terms of the prepulse shock location. Both the main shock $m_{s}^{M}$ and the prepulse shock positions $m_{s}^{p}$ are governed by the Hugoniot relations

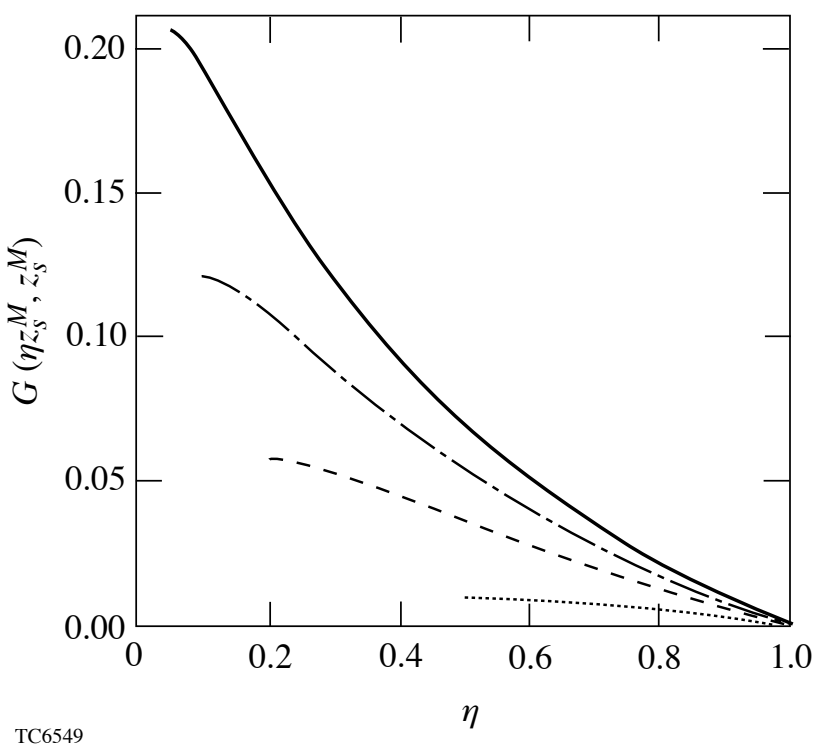

Figure 98.50

Plots of $G\left(\eta z_{s}^{M}, z_{s}^{M}\right)$ for $z_{s}^{M}=2,5,10,20$ (dotted, dashed, dash-dotted, and solid lines, respectively) show that $G$ does not exceed 0.21 for typical values of $z_{s}^{M} \leq 20$.

$$
\dot{m}_{s}^{M}=\sqrt{\frac{\gamma-1}{2} P_{f} \rho_{M}\left(\frac{m_{s}^{M}}{m_{s}^{p}}\right)^{\mu}\left[1+D\left(z_{s}^{M}\right)\left(z_{s}^{M}-1\right)\right]},
$$

$$
\dot{m}_{S}^{p}=\sqrt{\frac{\gamma-1}{2} P_{p} \rho_{p}\left(\frac{m_{*}}{m_{s}^{p}}\right)^{\delta}},
$$

where $\delta \simeq 1.315$. Replacing the time variable with $m_{s}^{p}(t)$ into Eq. (88) leads to the following algebraic equation relating the main and the prepulse shock location:

$$
\begin{aligned}
\left(z_{s}^{p}\right)^{\beta}-z_{\text {shell }}^{\beta}= & \beta \sqrt{\frac{2(\gamma-1)}{\gamma+1} \frac{P_{p}}{\omega(\gamma) P_{f}}} \\
& \times\left[\sigma\left(z_{s}^{M}\right)-\sigma\left(z_{\text {shell }}\right)\right]
\end{aligned}
$$

where $\beta=(\delta-\mu+2) / 2, \quad z_{s}^{p}=m_{s}^{p} / m_{*}, \quad z_{\text {shell }}=m_{\text {shell }} / m_{*}$, and 


$$
\sigma(\xi)=\sqrt{\frac{(\mu-2) \xi^{1-\mu}-(\mu-1) \xi^{2-\mu}+1}{(\mu-2)(\mu-1)}} .
$$

It is important to recognize that Eq. (90) has been derived using the condition that $z_{s}^{p}=z_{\text {shell }}$ when $z_{s}^{M}=z_{\text {shell }}$. This is an essential constraint requiring that both the prepulse and main shock merge on the shell's inner surface. Observe that Eq. (90) can be used to find $z_{s}^{p}$ in terms of $z_{s}^{M}$. An analytic form of $z_{s}^{M}$ in terms of $z_{s}^{p}$ can also be found by approximating $\mu \simeq 1.45$ and reducing Eq. (90) to a second-order algebraic equation for $z_{s}^{M}$.

Similar to the analysis in Shock Propagation Through a Relaxed Profile of the First Kind (p. 112), we estimate the dynamic corrections to the entropy profile by rewriting the entropy at the main shock front:

$$
S\left(z_{s}^{M}\right)=\left(\frac{z_{s}^{p}}{z_{s}^{M}}\right)^{\mu \gamma} \frac{P_{\mathrm{ps}}^{M}\left(z_{s}^{M}, t\right)}{\rho_{M}^{\gamma}} .
$$

Since we did not find an exact solution of the post-shock pressure for the static case, it is not worth calculating small corrections to an already inexact solution. Nevertheless, we retain the corrections due to the dynamic evolution of the relaxed profile. These corrections require including the time dependence of $z_{s}^{p}$ in the pre-shock density. We speculate that the largest corrections to the entropy are likely to come from such dynamic effects. This consideration is supported by the large power index $\mu \gamma \simeq 2.4$ for $z_{s}^{p}$ in Eq. (92) and the finite shock strength corrections of the order of $\sqrt{P_{p} / P_{f}}$ in Eq. (92) for $z_{s}^{p}$. It follows that the dynamic corrections to the entropy profile can be determined by substituting $z_{s}^{p}$ from Eq. (90) into Eq. (92) and by replacing $z_{s}^{M}$ with $z=m / m_{*}$. A straightforward manipulation leads to the following form of the entropy profile:

$$
\begin{aligned}
S(m)= & S_{\infty}(m)\left\{1-\beta\left(\frac{m_{*}}{m_{\text {shell }}}\right)^{\beta} \sqrt{\frac{2(\gamma-1)}{(\gamma+1) \omega(\gamma)} \frac{P_{p}}{P_{f}}}\right. \\
& \left.\times\left[\sigma\left(\frac{m_{\text {shell }}}{m_{*}}\right)-\sigma\left(\frac{m}{m_{*}}\right)\right]\right\}^{\frac{\gamma \mu}{\beta}},
\end{aligned}
$$

where $S_{\infty}$ is given in Eq. (84). Observe that dynamic corrections to the adiabat shape lead to a shallower profile.

\section{Conclusion}

We have derived analytical forms of the relaxation adiabat shapes for (1) the case where the prepulse is long enough that the rarefaction wave catches the prepulse shock at the rear surface of the shell, and (2) the case of short prepulses, where the mass undertaken by the unattenuated prepulse shock is less than the total mass of the shell. The analytic relaxation adiabat profiles derived here are in excellent agreement with simulation. In addition, we have shown that relaxation designs with short prepulses lead to steeper adiabat gradients than decaying shock designs. The effects of mass ablation and residual ablation pressure on relaxation adiabat shapes will be analyzed in a future article.

\section{ACKNOWLEDGMENT}

This work was supported by the U.S. Department of Energy Office of Inertial Confinement Fusion under Cooperative Agreement No. DE-FC0392SF19460, the University of Rochester, and the New York State Energy Research and Development Authority. The support of DOE does not constitute an endorsement by DOE of the views expressed in this article.

\section{Appendix A: Relaxed Density Profiles in Real Space}

A better understanding of the relaxed profile shape can be obtained by converting the profile functions from the Lagrangian coordinate $m$ to the spatial coordinate $x$. Equation (3) relating the mass to the initial density is also valid if the initial density is replaced by the density at time $t$ as long as the lower limit and upper limit of integration are the trajectory of the outer surface $x_{\text {out }}(t)$ and the trajectory of generic fluid element $x(t)$. It follows that the conversion between mass and real space is straightforward once the relation between $m$ and $x$ is rewritten in the differential form

$$
\frac{d m}{d x}=\rho(x, t) .
$$

Equation (A1) is then used to rewrite the profiles of the first kind [Eq. (18)] and second kind [Eqs. (43) and (44)] in real space.

\section{Relaxed Profiles of the First Kind}

In the case where the shock and rarefaction merge at the inner shell surface, one can substitute Eq. (A1) into (18) and find the density profile shape in real space:

$$
\rho=\rho_{p}\left[\frac{\gamma-1}{\gamma+1} \frac{x-x_{\mathrm{out}}}{a_{p} t}\right]^{\frac{2}{\gamma-1}},
$$


where $x_{\text {out }}$ is the trajectory of the outer shell surface coinciding with the trailing edge of the rarefaction wave moving away from the shell with the escape velocity $2 a_{p} /(\gamma-1)$. The range of $x$ in Eq. (A2) is limited by the trailing and leading edge of the rarefaction wave. Since the leading edge travels inside the shell with the sound speed, the range of $x$ is limited by $x_{\text {out }}$ $<x<x_{\text {out }}+a_{p} t$. Note that $t=0$ at the time when the prepulse ends and the rarefaction is launched. Equation (A2) indicates that, in real space, the density profile is a simple power law of the distance from the rarefaction trailing edge. The leading edge of the rarefaction wave reaches the prepulse shock at the inner shell surface $\left(m_{*}=m_{\text {shell }}\right)$ at the time

$$
t_{\text {shell }}=\frac{m_{\text {shell }}}{\left(a_{p} \rho_{p}\right)}=\frac{\gamma-1}{\gamma+1} \frac{d_{\text {shell }}}{a_{p}}
$$

where $d_{\text {shell }}$ is the initial shell thickness and $m_{\text {shell }}=\rho_{0} d_{\text {shell }}$ is the total shell mass. At this time, the density profile is simply

$$
\rho\left(t=t_{\text {shell }}\right)=\rho_{p}\left(\frac{x-x_{\text {out }}}{d_{\text {shell }}}\right)^{\frac{2}{\gamma-1}} .
$$

Note that the profile extends over a distance equal to the uncompressed shell thickness $d_{\text {shell }}$ and approaches the compressed density $\rho_{p}$ on the shell's inner surface located at $x_{\text {in }}=x_{\text {out }}+d_{\text {shell }}$.

\section{Relaxed Profiles of the Second Kind}

By defining with $x_{*}(\tau)$ the trajectory of the Lagrangian point corresponding to the fluid element where the prepulse shock and rarefaction wave interact (i.e., $m=m_{*}$ ), and with $x_{s}^{p}(\tau)$ the location of the prepulse shock after the interaction, the spatial density profile for the region $x_{*}(\tau)<x<x_{s}^{p}(\tau)$ can be obtained by substituting Eq. (A1) into Eq. (44), leading to

$$
\rho=\rho_{p}\left[\frac{1}{1+4(\mu-1)\left(1-x / x_{s}^{p}\right)}\right]^{\frac{\mu}{\mu-1}},
$$

where $\mu=1.45$ for $\gamma=5 / 3$. By defining with $\bar{x}_{*} \equiv x_{*}(1)$ the initial position inside the shell of the shock-rarefaction interaction point, it is straightforward to show that

$$
\begin{gathered}
x_{s}^{p}(\tau)=\bar{x}_{*} z_{s}^{p}(\tau), \\
x_{*}(\tau)=\bar{x}_{*} z_{s}^{p}(\tau)\left[1+\frac{1-z_{S}^{p}(\tau)}{4(\mu-1)}\right],
\end{gathered}
$$

where $z_{s}^{p}$ is given in Eq. (31). Equation (A5) represents the spatial density profile of the shell portion between the shockrarefaction merging point and the shock front. The density profile of the remaining portion between the shock-rarefaction merging point and the outer shell surface is described by Eq. (43) and in real space can again be determined by integrating Eq. (43) upon substitution of Eq. (A1). The result is similar to the density profile of the first kind and yields

$$
\rho=\rho_{p}\left(\frac{\gamma-1}{\gamma+1} \frac{x-x_{\mathrm{out}}}{a_{p} t}\right)^{\frac{2}{\gamma-1}}
$$

It is important to emphasize that the density profiles [Eqs. (43) and (44)] are approximate solutions; therefore, the profiles [Eqs. (A5) and (A8)] are also approximate solutions. The location of the rarefaction trailing edge (or outer shell surface) cannot be exactly calculated because it is affected by the sound waves traveling down from the point of shock-rarefaction interaction. An approximate form of $x_{\text {out }}$ can be derived by requiring that the density is continuous at $x=x_{*}(\tau)$, thus setting Eq. (A8) equal to Eq. (A5) at $x *(\tau)$. This leads to the following form of $x_{\text {out }}$ :

$$
x_{\mathrm{out}}=x_{*}(\tau)-\bar{x}_{*} \frac{a_{p} \tau}{z_{S}^{p}(\tau)^{\frac{\mu(\gamma-1)}{2}}} .
$$

Observe that, in real space, the density profile is represented by two very different functions of $x$ : Eq. (A5) describes the profile behind the shock, and Eq. (A8) describes the profile behind the rarefaction-shock merging point.

\section{REFERENCES}

1. K. Anderson and R. Betti, Phys. Plasmas 10, 4448 (2003).

2. V. N. Goncharov, J. P. Knauer, P. W. McKenty, P. B. Radha, T. C. Sangster, S. Skupsky, R. Betti, R. L. McCrory, and D. D. Meyerhofer, Phys. Plasmas 10, 1906 (2003).

3. K. Anderson and R. Betti, Phys. Plasmas 11, 5 (2004). 


\section{Using Nuclear Data and Monte Carlo Techniques to Study Areal Density and Mix in $\mathrm{D}_{2}$ Implosions}

\section{Introduction}

Maximizing the hot-fuel areal density $\left(\rho R_{\text {hot }}\right)$ and understanding the effects of mix upon it are fundamental issues of inertial confinement fusion (ICF). ${ }^{1-3}$ One method used to estimate $\rho R_{\text {hot }}$ of $D_{2}$-filled capsule implosions is to measure the yields of secondary protons $\left(Y_{2 p}\right)$ and/or secondary neutrons $\left(Y_{2 n}\right)$ relative to the primary neutron yield $\left(Y_{1 n}\right) .{ }^{4-12}$ These secondary particles result from sequential reactions in which the energetic primary products of reactions

$$
\begin{gathered}
\mathrm{D}+\mathrm{D} \rightarrow n(2.45 \mathrm{MeV})+{ }^{3} \mathrm{He}(0.82 \mathrm{MeV}), \\
\mathrm{D}+\mathrm{D} \rightarrow p(3.02 \mathrm{MeV})+\mathrm{T}(1.01 \mathrm{MeV})
\end{gathered}
$$

undergo fusion reactions with thermal deuterons in the fuel:

$$
\begin{aligned}
{ }^{3} \mathrm{He}(\leq 0.82 \mathrm{MeV})+\mathrm{D} \rightarrow & p(12.5-17.4 \mathrm{MeV}) \\
& +{ }^{4} \mathrm{He}(6.6-1.7 \mathrm{MeV}) \\
\mathrm{T}(\leq 1.01 \mathrm{MeV})+\mathrm{D} \rightarrow & n(11.9-17.2 \mathrm{MeV}) \\
& +{ }^{4} \mathrm{He}(6.7-1.4 \mathrm{MeV})
\end{aligned}
$$

These processes produce secondary particles with spectra spread over significant energy intervals due to the kinetic energy of the primary reactants. The secondary-particle yields are typically two to three orders of magnitude lower than the primary yield, and the ratios $Y_{2 n} / Y_{1 n}$ and $Y_{2 p} / Y_{1 n}$ (which are linearly dependent on $\rho R_{\text {hot }}$ in certain plasma regimes) can each be used to infer a value of $\rho R_{\text {hot }}$ for implosions of $\mathrm{D}_{2}$-filled capsules in both direct- and indirect-drive experiments. ${ }^{12-15}$ In those studies, the simple "hot-spot" and/or the "uniform" models were used to relate these ratios to $\rho R_{\text {hot }}$.
Although these simple models have been widely used to infer a value of $\rho R_{\text {hot }}$, they have some serious limitations that can result in misinterpretation and errors (as described in the next section); one manifestation of these problems is often disagreement between the proton- and neutron-inferred values of $\rho R_{\text {hot }}$ calculated from experimental data (see Fig. 98.51). These deviations are related to a combination of mix, temperature profile, and the difference between the cross section for secondary reactions (3) and (4). These factors can cause secondary protons and neutrons to be produced in different regions of the compressed capsules (Fig. 98.52). In addition,

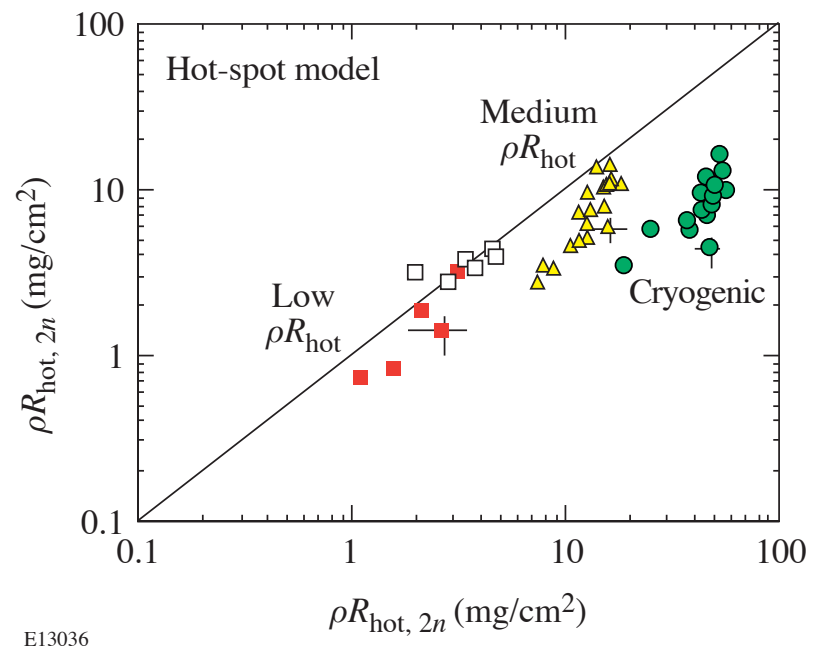

Figure 98.51

Secondary-proton- and secondary-neutron-implied values of $\rho R_{\text {hot }}$ are compared for implosions of low $\rho R_{\text {hot }}$ (squares), medium $\rho R_{\text {hot }}$ (triangles), and cryogenic (circles) capsules on OMEGA. For low- $\rho R_{\text {hot }}$ implosions, the values of $\rho R_{\text {hot }}$ inferred from secondary protons and neutrons using the simple hot-spot model agree well. It is also shown that values of $\rho R_{\text {hot }}$ are larger for implosions with $\sim 12$-kJ laser energy (open squares) than for implosions with $\sim 23-\mathrm{kJ}$ laser energy (closed squares). For these dramatically overdriven implosions, it is possible that the effects of mix are coming back into play, as indicated by the observation that $\rho R_{\text {hot }, 2 n}$ is larger than $\rho R_{\text {hot, } 2 p}$. For implosions with larger $\rho R_{\text {hot }}$, however, the values inferred from secondary neutrons are always larger than the values from secondary protons. The error bars shown are typical of each type of implosion; they include uncertainties in the measurements and in the assumed values of the density. 

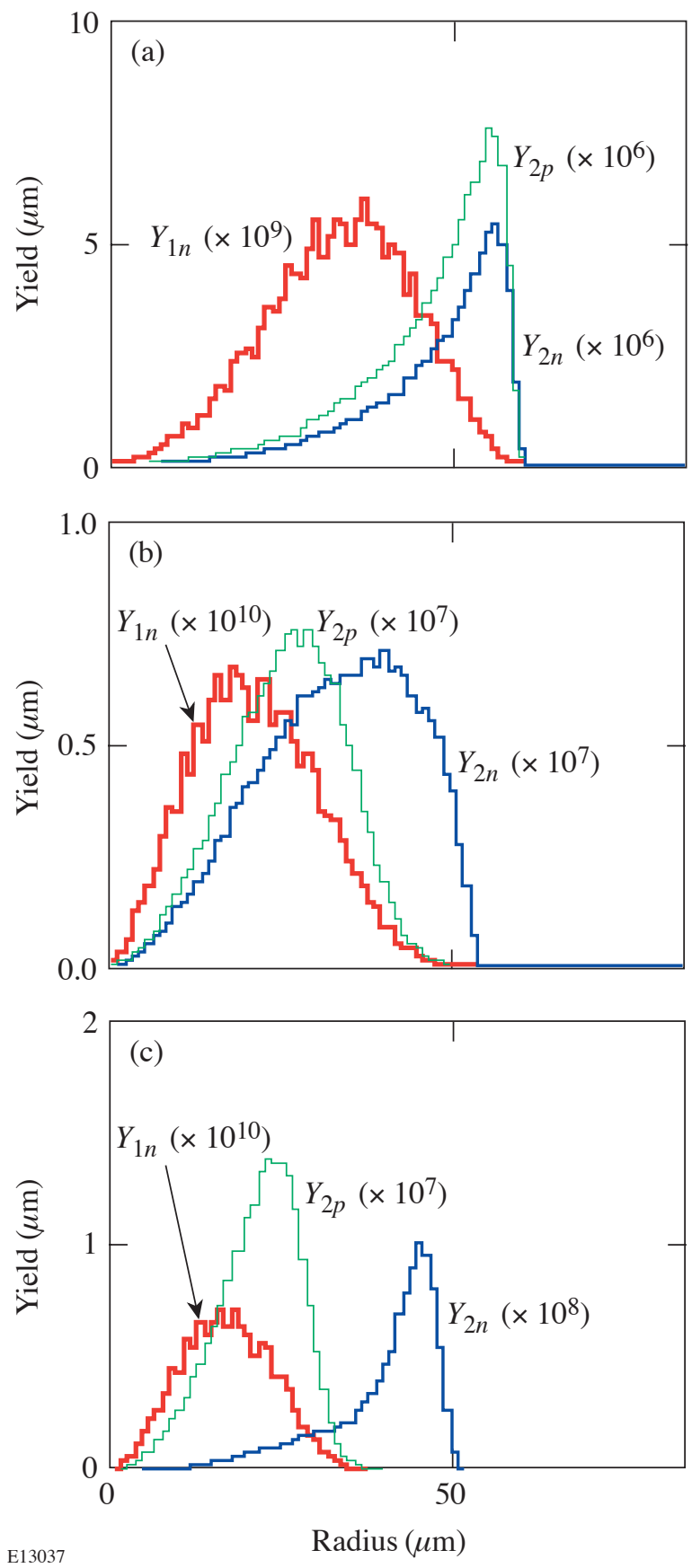

Figure 98.52

Calculated radial distributions of primary- and secondary-birth positions per unit length for (a) low- $\rho R$ implosion 30981, (b) medium- $\rho R$ implosion 27443 , and (c) cryogenic implosion 28900. For low- $\rho R$ implosions, where $\rho R_{2 p}$ and $\rho R_{2 n}$ agree reasonably well, birth positions of secondary protons and neutrons are virtually identical. For medium- $\rho R$ and cryogenic implosions, however, where $\rho R_{2 n}$ is always larger than $\rho R_{2 p}$, secondary neutrons are produced in more outer regions compared to secondary protons. Note that calculated radial distributions of primary birth rates per unit volume (as opposed to unit radius) are shown in Figs. 98.60-98.62 for these three implosions. others have noted some puzzling issues with recent secondaryneutron measurements in indirect-drive implosions on OMEGA. ${ }^{16}$ In that work, the authors observed a factor-of-3larger $Y_{2 n} / Y_{1 n}$ ratio and a narrower secondary-neutron spectrum than predicted for these low-convergence implosions (where mix should be relatively unimportant). In contrast, for high-convergence implosions, they found better agreement between measured and predicted $Y_{2 n} / Y_{1 n}$ values.

In previous work, ${ }^{12}$ high-resolution secondary-proton spectra were obtained during experiments on OMEGA. ${ }^{17}$ The yields were used with measured neutron yields to estimate $\rho R_{\text {hot }}$ with the hot-spot and uniform models, and it was shown that the $Y_{2 p} / Y_{1 n}$-inferred $\rho R_{\text {hot }}$ was often lower than the $Y_{2 n} / Y_{1 n}$-inferred $\rho R_{\text {hot }}$. This was attributed to the effects of fuel-shell mix, and it was suggested that the two inferences might be considered lower and upper limits, respectively. In this article, that work is extended to cover a wider range of implosion types and to include Monte Carlo simulations that allow a detailed study of the implications of more-realistic models of the compressed core on the secondary production. The following sections (1) describe the hot-spot and uniform models and their limitations, (2) describe the experiments and the range of measured parameters, (3) describe a Monte Carlo program that will model the implosions to understand how particle production occurs, (4) discuss results from both experiments and Monte Carlo calculations, with an emphasis on how $\rho R_{\text {hot }}$ is related to the yields of primary and secondary particles, and (5) summarize the results.

\section{Primary and Secondary Products}

The hot-spot and uniform models have been commonly used to relate $Y_{2 p} / Y_{1 n}$ and $Y_{2 n} / Y_{1 n}$ to $\rho R_{\text {hot }}$. The hot-spot model assumes that an imploded capsule is a sphere of uniform density and temperature and that all primary reactions occur at the very center of the capsule. A fraction of the primary ${ }^{3} \mathrm{He}$ (tritons) fuse with thermal deuterons, producing secondary protons (neutrons) as they move radially outward. As the primary particles travel through the D plasma, they lose energy, and the probability for producing secondary particles along the path varies greatly since the secondary $\mathrm{D}^{3} \mathrm{He}$ and DT fusion cross sections ( $\sigma_{\mathrm{D}^{3} \mathrm{He}}$ and $\left.\sigma_{\mathrm{DT}}\right)$ are strong functions of the primary ${ }^{3} \mathrm{He}$ and Tenergies [Fig. 98.53(a) $] .{ }^{18} \sigma_{\mathrm{D}^{3} \mathrm{He}}$ peaks at $\sim 0.65 \mathrm{MeV}$, close to the ${ }^{3} \mathrm{He}$ birth energy $(0.8 \mathrm{MeV})$, while $\sigma_{\mathrm{DT}}$ peaks at $\sim 0.18 \mathrm{MeV}$, significantly lower than the triton birth energy $(1.0 \mathrm{MeV})$. As a result, secondary protons are mainly produced near the ${ }^{3} \mathrm{He}$ birth position, while secondary neutrons are mainly produced farther away from the triton birth position [see Fig. 98.53(b)]. This information is used to calcu- 
late $\rho R_{\text {hot }}$ from $Y_{2 p} / Y_{1 n}$ and $Y_{2 n} / Y_{1 n}$, and the resulting dependencies are shown in Fig. 98.54 for D plasmas with different temperatures and densities. The ratios each saturate at different values of $\rho R_{\text {hot }}$ for different temperatures and densities because the primary ${ }^{3} \mathrm{He}$ and tritons generally have significantly different ranges in the plasma. If either particle stops before leaving the fuel, it will not sample the entire $\rho R_{\text {hot }}$, and the implied value of $\rho R_{\text {hot }}$ underestimates the actual value. $Y_{2 p} / Y_{1 n}$ does not depend on temperature until it starts to saturate, while $Y_{2 n} / Y_{1 n}$ is sensitive to temperature well below the saturation level. Therefore, without a reasonable estimate of plasma temperature, $Y_{2 n} / Y_{1 n}$ cannot be used to accurately infer $\rho R_{\text {hot }}$.

The uniform model assumes that the primary particles are produced uniformly in a sphere of constant density and temperature. The $Y_{2 p} / Y_{1 n}$ and $Y_{2 n} / Y_{1 n}$ dependencies show similar behavior to the hot-spot model. The primary difference is that values of $\rho R_{\text {hot }}$ implied by the uniform model are always larger than values from the hot-spot model because the mean path length of primary particles in the D plasma is shorter by $25 \%$ in the uniform model, when saturation has not occurred. The simulations described in the Results section (p. 128) indicate that the hot-spot model gives more-meaningful values of $\rho R_{\text {hot }}$ than the uniform model; therefore, the hot-spot model will be used throughout the remainder of this article.

Both models have limitations that can introduce errors into the analysis of $\rho R_{\text {hot }}$. These include the saturation of $Y_{2 p}$ and $Y_{2 n}$ and the uncertainty introduced by the temperature dependence of $Y_{2 n}$. The shapes of temperature and density profiles and the presence of fuel-shell mix $^{20-22}$ can have substantial impact on secondary-particle production. In reality, the temperature is highest and the density is lowest at the center of the implosion. As the temperature decreases and the density increases, the rate of energy loss of primary particles becomes larger. This typically causes a reduction of the secondaryproton production rate and an enhancement of the secondaryneutron production rate [see Fig. 98.53(a)]. Fuel-shell mix
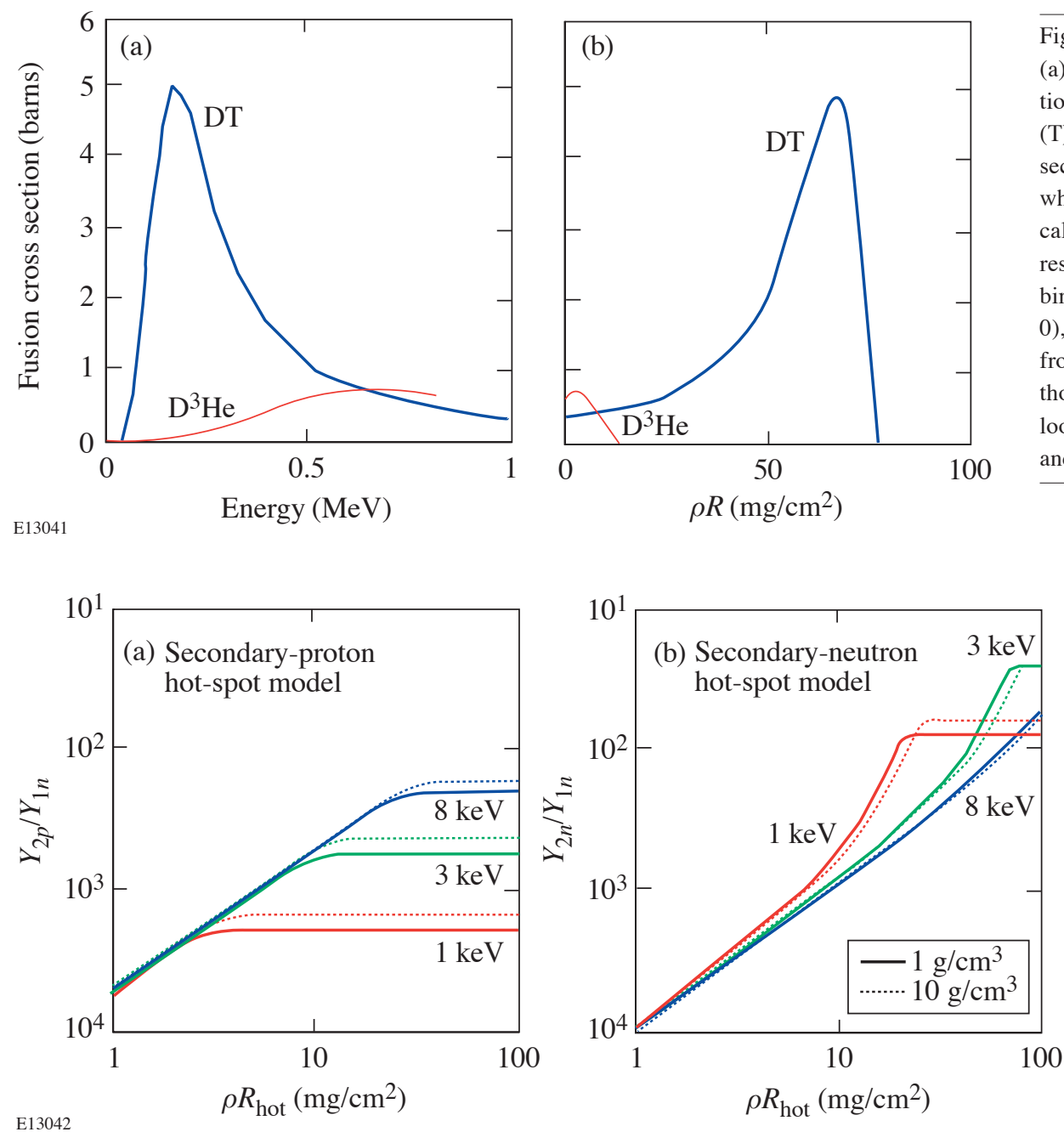

Figure 98.53

(a) Dependence of the secondary $\mathrm{D}^{3} \mathrm{He}$ (DT) reaction cross section on the energy of the primary ${ }^{3} \mathrm{He}$ (T) in a cold D plasma. ${ }^{18}$ The $\mathrm{D}^{3} \mathrm{He}$-reaction cross section is peaked close to the birth energy of ${ }^{3} \mathrm{He}$, while the DT-reaction cross section peaks dramatically after $\mathrm{T}$ has lost most of its energy. (b) As a result, secondary protons are created close to the birth points of primary ${ }^{3} \mathrm{He}$ (here defined as $\rho R=$ 0 ), while secondary neutrons are produced away from the birth points of primary $\mathrm{T}(\rho R=0)$. Although this plot is for a 1-g/cc, 3-keV D plasma, it looks similar for plasmas with different densities and temperatures. 
lowers the temperature in the mix region, which increases the energy loss rate and results in a further reduction of the secondary-proton production rate and an enhancement of the secondary-neutron production rate. Shell material mixed into the fuel can directly affect secondary production by increasing the energy lost by $\mathrm{T}$ and ${ }^{3} \mathrm{He}$ after traveling through a given amount of $\mathrm{D}$, due to the higher effective charge of the shell material mixed in.

\section{Experiments}

In the direct-drive experiments described here, distributed phase plates, ${ }^{23}$ polarization smoothing using birefringent wedges, ${ }^{24}$ and 1 -THz, two-dimensional smoothing by spectral dispersion ${ }^{25}$ were applied to smooth the OMEGA laser beams in order to enhance implosion uniformity and the nuclear reaction rate. Three types of capsules were used to study implosions with a wide range of areal densities: Low- $\rho R_{\text {hot }}$ implosions were studied using thin $(\sim 3-\mu \mathrm{m})$ glass $\left(\mathrm{SiO}_{2}\right)$ shells filled with $\sim 15$ atm of $\mathrm{D}_{2}$. Some of these capsules were irradiated with a 1-ns square pulse delivering $23 \mathrm{~kJ}$ of on-target energy, while others were irradiated with a shorter (600- to 800 -ps) pulse with on-target energy of $\sim 12 \mathrm{~kJ} .{ }^{26}$ Medium- and large- $\rho R$ implosions were studied using capsules with thick $(\sim 20-\mu \mathrm{m})$ plastic $(\mathrm{CH})$ shells filled with $\sim 15 \mathrm{~atm}$ of $\mathrm{D}_{2}$ and cryogenic capsules with an $\sim 100-\mu$ m layer of $\mathrm{D}_{2}$ ice enclosed within a 3- to $\sim 5-\mu \mathrm{m}$-thick $\mathrm{CH}$ shell, respectively. They were all irradiated with 1-ns square pulses, delivering $23 \mathrm{~kJ}$ of ontarget energy.

Charged-particle data were collected with two types of spectrometers: Wedged-range-filter proton spectrometers ${ }^{12,27}$ provided secondary-proton spectra from up to six different directions simultaneously. These spectra were used to calculate the yield and mean energy of secondary protons. Two magnet-based charged-particle spectrometers ${ }^{27}$ provided the spectra of primary protons and tritons for low- $\rho R$ implosions. Neutron data were obtained from three diagnostics: Neutron time-of-flight detectors ${ }^{28}$ provided primary- and secondaryneutron yields as well as primary-neutron-yield-averaged ion temperature $\left(\left\langle T_{i}\right\rangle_{Y_{1 n}}\right)$, and a neutron temporal diagnostic ${ }^{29}$ measured the peak primary-neutron production time and the DD burn duration. In addition, secondary-neutron spectra were obtained from the 1020 -scintillator array ${ }^{30}$ on some of the more-recent implosions.

The data from each implosion then include the five quantities $Y_{1 n}, Y_{2 n}, Y_{2 p},\left\langle T_{i}\right\rangle_{Y_{1 n}}$, and $\left\langle E_{2 p}\right\rangle$, which will be matched to simulations in the next section. In addition, the spectral energy distributions of the secondary protons (and sometimes second- ary neutrons) will be compared with the simulations. The yields and $\left\langle T_{i}\right\rangle_{Y_{1 n}}$, together with a realistic plasma density, can also be used to determine what the simple hot-spot and uniform models imply for values of $\rho R_{\mathrm{hot}, 2 p}^{\mathrm{expl}}$ and $\rho R_{\mathrm{hot}, 2 n}^{\mathrm{expl}}$ (where the superscript "exp1" refers to the use of the measured $\left\langle T_{i}\right\rangle_{Y_{1 n}}$ as the characteristic ion temperature).

\section{Monte Carlo Simulations}

A Monte Carlo program was developed to model the experiments described in the previous section. This allows us to use more-realistic temperature and density profiles than those in the hot-spot and uniform models. The burn-averaged ion temperature profile $\left[T_{i}(r)\right]$ and the shell (or cold fuel, for cryogenic capsules) density profile $\left[\rho_{\text {cold }}(r)\right]$ are assumed to have super- or sub-Gaussian profiles, and the six input parameters are $T_{i 0}, T_{i w}, T_{i p}, S_{r 0}, S_{w}$, and $S_{p}$ characterizing the temperature and density profiles

$$
T_{i}(r)=T_{i 0} \exp \left[-\left(r / T_{i w}\right)^{T_{i p}}\right]
$$

and

$$
\rho_{\text {cold }}(r)=\rho_{\text {cold } 0} \exp \left\{-\left[\left(r-S_{r 0}\right) / S_{w}\right]^{S_{p}}\right\} .
$$

These parameters are varied to produce simulated particle production that best fits the measured data for each implosion. The hot-fuel density profile $\left[\rho_{\text {hot }}(r)\right]$ is calculated assuming that the plasma is isobaric out to the peak shell pressure region; with this constraint, $\rho_{\text {cold } 0}$ is then adjusted to conserve the fuel mass. (The initial fuel mass is calculated based on the initial fuel pressure and the size of the capsule.)

For computational purposes, each primary particle is assumed to produce a secondary particle, and a spectrum of particles per unit energy $d N_{2} / d E$ is obtained. Since only a small fraction of the primary particles actually undergo secondary reactions, the secondary yield and spectrum need to be normalized according to $Y_{2}=\left\langle P_{2}\right\rangle Y_{1}$ and

$$
\begin{aligned}
d Y_{2} / d E & \approx\left\langle P_{2}\right\rangle Y_{1}\left(d N_{2} / d E\right) / N_{2} ; \\
\left\langle P_{2}\right\rangle & \equiv\left\langle\int n_{\mathrm{D}}(l) \sigma_{\mathrm{sec}}(l) d l\right\rangle
\end{aligned}
$$

is the probability of primary-to-secondary conversion, calculated in the program as the primary-yield-weighted mean value of the line integral of the D number density $\left(n_{\mathrm{D}}\right)$ times the secondary fusion cross section $\left(\sigma_{\mathrm{sec}}\right)$ for all possible primaryparticle trajectories. The primary-particle production is determined by the density and temperature profiles. The particles are followed along their trajectories through the capsule until 
they either escape or lose all of their energy. The energy loss is calculated, as briefly described in the next paragraph, in order to obtain $\sigma_{\mathrm{sec}}$ along paths of primary particles. The probability of a secondary fusion reaction is calculated along the path of the primary particle; then the birth position, direction, and energy of the secondary particle are calculated. The radial distributions of the primary- and secondary-particle birth positions are recorded as well to illustrate the effects of profiles and fuel-shell mix.

The energy loss of charged particles in plasmas is strongly dependent upon the velocity of the particle. Depending on the relative magnitude of the particle velocity $v_{p}$ and the thermal velocity $v_{\text {th }}$ of the background electrons, the plasma can be described as "cold" $\left(v_{p}>>v_{\text {th }}\right)$, "warm" $\left(v_{p} \sim v_{\text {th }}\right)$, or "hot" $\left(v_{p} \ll<v_{\text {th }}\right)$. The theory described in Ref. 19 predicts that the plasma-stopping power reaches a maximum when $v_{p} \approx v_{\text {th }}$, which was also demonstrated for the first time in Ref. 39. The general form for the charged-particle energy loss per unit distance, $d E / d x$, in fully ionized plasmas is given by ${ }^{19}$

$$
-\frac{1}{Z^{2}} \frac{d E}{d X}=\left(\frac{\omega_{p} e}{v_{p}}\right)^{2} G\left(\frac{v_{p}^{2}}{v_{\mathrm{th}}^{2}}\right) \ln \Lambda,
$$

where $\omega_{p}=\left(4 \pi n_{e} e^{2} / m_{e}\right)^{1 / 2}$ is the electron-plasma frequency, $Z$ is the projectile charge number, $v_{p}$ is the velocity of the particle, $v_{\text {th }}=\left(2 T / m_{e}\right)^{1 / 2}$ is the thermal velocity of the plasma electrons, and $\ln \Lambda$ is the Coulomb logarithm; $n_{e}, e$, and $m_{e}$ are the electron density, charge, and mass, respectively. $G\left(v_{p} / v_{\text {th }}\right)$ is the Chandrasekhar function, which peaks at $v_{p} / v_{\text {th }} \approx 1$, and explains why the stopping power reaches a maximum when $v_{p} \approx v_{\text {th }}$. The formulation given above applies to both plasmaelectron stopping and plasma-ion stopping, where the latter, for the conditions of interest here, is about $10 \%$ of the stopping by electrons.

Since the model is static, the primary yield is calculated by multiplying the burn profile by the burn duration (full width at half maximum of the neutron-production rate); therefore, the error in the measurement of the burn duration is included in the error of the primary yield. $\left\langle E_{2 p}\right\rangle$ is calculated from the secondary-proton spectrum, and $\left\langle T_{i}\right\rangle_{Y_{1 n}}$ is determined in the region where the primary particles are produced. ${ }^{31}$ Each of the six input parameters is varied over a large range, initially using large steps to identify the region of small $\chi^{2}$. This region is then more carefully explored using finer grids; as a result, the sixdimensional parameter space is explored completely. For each set of model parameters, the predicted values of the experi- mentally measured quantities are calculated and the quality of agreement with the data from a particular implosion is characterized with the total $\chi^{2}$, which takes into account uncertainties in the experimental measurements. For each implosion, it is found that multiple local minima exist within the space of model parameters but there is one clear region with the smallest values of $\chi^{2}$. Errors in the values of individual model parameters are then estimated by asking how much they can be changed without causing the total $\chi^{2}$ to increase by more than 1 . Although the widths and shapes of secondary-proton spectra are not used as fit criteria, it will be seen that the predicted spectra match the measured spectra quite well; this fact provides extra confidence that the best-fit-model parameters are realistic.

The characteristics of the best-fit model for each implosion were used to determine how realistic the hot-spot-model inferred values of $\rho R_{\text {hot }}$ are. Values of $Y_{2 p} / Y_{1 n}, Y_{2 n} / Y_{1 n},\left\langle T_{i}\right\rangle_{Y_{1 n}}$, and plasma density from the simulations were used to infer $\rho R_{\mathrm{hot}, 2 p}^{\mathrm{sim} 1}$ and $\rho R_{\mathrm{hot}, 2 n}^{\mathrm{sim} 1}$ according to Fig. 98.54 (the superscript "sim1" indicates that $\left\langle T_{i}\right\rangle_{Y_{1 n}}$ was used as the characteristic ion temperature). The values of $\rho R_{\mathrm{hot}, 2 p}^{\mathrm{sim} 2}$ and $\rho R_{\mathrm{hot}, 2 n}^{\mathrm{sim} 2}$ were calculated assuming that the appropriate temperatures are averages weighted by secondary yields $\left\langle T_{i}\right\rangle_{Y_{2 p}}$ and $\left\langle T_{i}\right\rangle_{Y_{2 n}}$, respectively. These values were then compared with $\rho R_{\text {hot }}^{\text {int }} \equiv \int \rho_{\mathrm{D}} d r$, integrated over the hotfuel region.

\section{Results}

\section{Low-Areal-Density Implosions}

For low- $\rho R_{\text {hot }}$ implosions, the primary ${ }^{3} \mathrm{He}$ and $\mathrm{T}$ traverse the entire hot-fuel region, and the values of $\rho R_{\text {hot }}$ inferred from secondary protons and neutrons using the hot-spot (or uniform) model generally agree with each other and usually give a reasonable estimate of the actual value of $\rho R_{\text {hot }}$. This is shown experimentally by the square points in Fig. 98.51, which compares values of $\rho R_{\mathrm{hot}, 2 p}^{\exp 1}$ and $\rho R_{\mathrm{hot}, 2 n}^{\exp 1}$. These values were inferred according to Fig. 98.54 assuming a $D$ plasma with a temperature of $\left\langle T_{i}\right\rangle_{Y_{1 n}} \mathrm{keV}$ and a density of $1.5 \mathrm{~g} / \mathrm{cc}$ (obtained from a typical best-fit simulation, as discussed below). Figure 98.51 also illustrates that $\rho R_{\mathrm{hot}, 2 p}^{\exp 1}$ and $\rho R_{\mathrm{hot}, 2 n}^{\exp 1}$ are larger for implosions with lower $(\sim 12 \mathrm{~kJ})$ on-target laser energy (open squares) than for implosions with full ( $23 \mathrm{~kJ})$ laser energy (closed squares). This could be explained by a larger amount of glass shell being ablated away in full-energy implosions, resulting in less material to drive the fuel in$\operatorname{ward}^{32,33}$ (Fig. 98.55). In addition, these values of $\rho R_{\text {hot }}^{\exp 1}$ from $D_{2}$ implosions with full laser energy show reasonable agreement with values from similar thin-glass-shell DT 
implosions, ${ }^{34,12}$ for which the knock-on method $^{35}$ was used to determine the $\rho R_{\text {hot }}$.

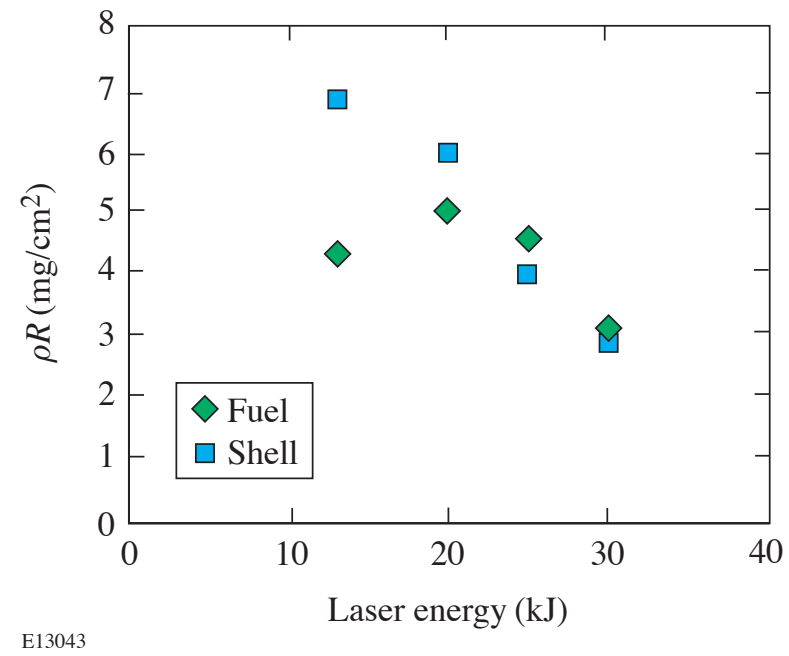

Figure 98.55

One-dimensional clean LILAC simulations for low- $\rho R$ implosion 30981 indicate that hot-fuel $\rho R$ starts to decrease as the capsule is significantly overdriven. This trend agrees with measurements where $\rho R_{\text {hot }}$ is lower for full-laser-energy-driven $(\sim 23-\mathrm{kJ})$, thin-glass-shell capsules than for lowlaser-energy-driven ( 12-kJ) capsules (Fig. 98.51).

For implosion 30981, which involved a 3.1- $\mu \mathrm{m}$ glass shell filled with $14.7 \mathrm{~atm}$ of $\mathrm{D}_{2}$ gas, Fig. 98.56(a) shows simulated density and temperature profiles from the best-fit simulation. Figure 98.56(b) shows radial distributions of the primary- and secondary-particle-birth positions; secondary protons and neutrons are produced in virtually identical regions of the capsule. In addition, a high plasma temperature and a low $\rho R_{\text {hot }}$ result in similar values of $\rho R_{\mathrm{hot}}^{\text {sim1 }}$ inferred from the simulated secondary yields. Values of $\rho R_{\mathrm{hot}}^{\mathrm{sim} 1}$ are inferred using the hot-spot model and assuming a plasma temperature of $\left\langle T_{i}\right\rangle_{Y_{1 n}} \mathrm{keV}$ and a plasma density of $1.5 \mathrm{~g} / \mathrm{cc}$ (obtained from simulation). In addition, values of $\rho R_{\mathrm{hot}}^{\mathrm{sim} 1}$ agree with $\rho R_{\mathrm{hot}}^{\mathrm{int}}$ obtained from the fuel-density profile shown in Fig. 98.56(a); this indicates that the small amount of fuel-shell mix in this type of implosion does not have much impact on the accuracy of the simple model. Results of the simulation along with measured data are summarized in Table 98.IV.

Simulated secondary spectra are in good agreement with measured spectra as shown in Figs. 98.56(c) and 98.56(d). The measured secondary-proton spectrum is an average of five spectra obtained simultaneously at different angles from implosion 30981.

\section{Medium-Areal-Density Implosions}

Correctly inferring the value of $\rho R_{\text {hot }}$ is more difficult for implosions of capsules with thick plastic shells because $Y_{2 p}$ reaches saturation when $\rho R_{\text {hot }}$ is sufficiently large, and $Y_{2 n}$ is enhanced in the presence of increased fuel-shell mix. The triangles in Fig. 98.51 show that the values of $\rho R_{\mathrm{hot}, 2 p}^{\exp 1}$ are often smaller than the values of $\rho R_{\mathrm{hot}, 2 n}^{\exp 1}$, as previously reported in Ref. 12. Values of $\rho R_{\mathrm{hot}, 2 p}^{\exp 1}$ and $\rho R_{\mathrm{hot}, 2 n}^{\exp 1}$ are inferred assuming a temperature of $\left\langle T_{i}\right\rangle_{Y_{1 n}} \mathrm{keV}$ and a D plasma with a density of $2 \mathrm{~g} / \mathrm{cc}$.

Figure 98.57(a) shows the temperature and density profiles that result in the best fit to the measured data for implosion 27443 (19.4- $\mu$ m plastic shell filled with 15 atm of $D_{2}$ gas), and Fig. 98.57(b) shows the resulting radial distributions of primary- and secondary-particle-birth positions. About 32\% of the initial $\mathrm{CH}$ mass remains, and $\sim 1.3 \mu \mathrm{m}$ of the initial $\mathrm{CH}$ layer has mixed into the fuel (which is similar to the amount of mix reported in Refs. 20-22). ${ }^{36}$ The ${ }^{3} \mathrm{He}$ are ranged out before traversing the entire fuel region. Figure 98.57(b) also illustrates an enhancement of $\mathrm{Y}_{2 n}$ by fuel-shell mix; the increased energy loss of T per unit $\rho R_{\text {hot }}$, due to the cooler, dense shell material, results in an enhanced DT fusion cross section (Fig. 98.53), which causes $Y_{2 n} / Y_{1 n}$ to overestimate $\rho R_{\text {hot }}^{\text {int }}$. In addition, $Y_{2 n} / Y_{1 n}$ is more sensitive to temperature in this $\rho R_{\text {hot }}$ range; using $\left\langle T_{i}\right\rangle_{Y_{1 n}}$, which is always higher than $\left\langle T_{i}\right\rangle_{Y_{2 n}}$, results in a larger inferred value of $\rho R_{\text {hot }}$.

Simulated yields and additional parameters characterizing the implosion are summarized and compared with measurements in Table 98.V. This table shows that the values of $\rho R_{\mathrm{hot}}^{\mathrm{sim} 1}$ implied by secondary protons and neutrons are smaller and larger than the value of $\rho R_{\mathrm{hot}}^{\mathrm{int}}$, respectively. The hot-spot model was used to obtain values of $\rho R_{\text {hot }}$ using $\left\langle T_{i}\right\rangle_{Y_{1 n}} \mathrm{keV}$ for the temperature and assuming the density of the $\mathrm{D}$ plasma was $2 \mathrm{~g} / \mathrm{cc}$.

The simulated secondary-proton spectrum is compared with the measured spectrum in Fig. 98.57(c). The measured secondary-proton spectrum is an average of three spectra simultaneously obtained at different angles from implosion 27443 and shows more downshift than spectra from the low$\rho R_{\text {hot }}$ implosions. The widths of the secondary-proton and secondary-neutron spectra [Fig. 98.57(d)] are slightly narrower than in the previous case because the average energy of the primary particle, at the time it undergoes secondary fusion, is smaller. ${ }^{12}$ 

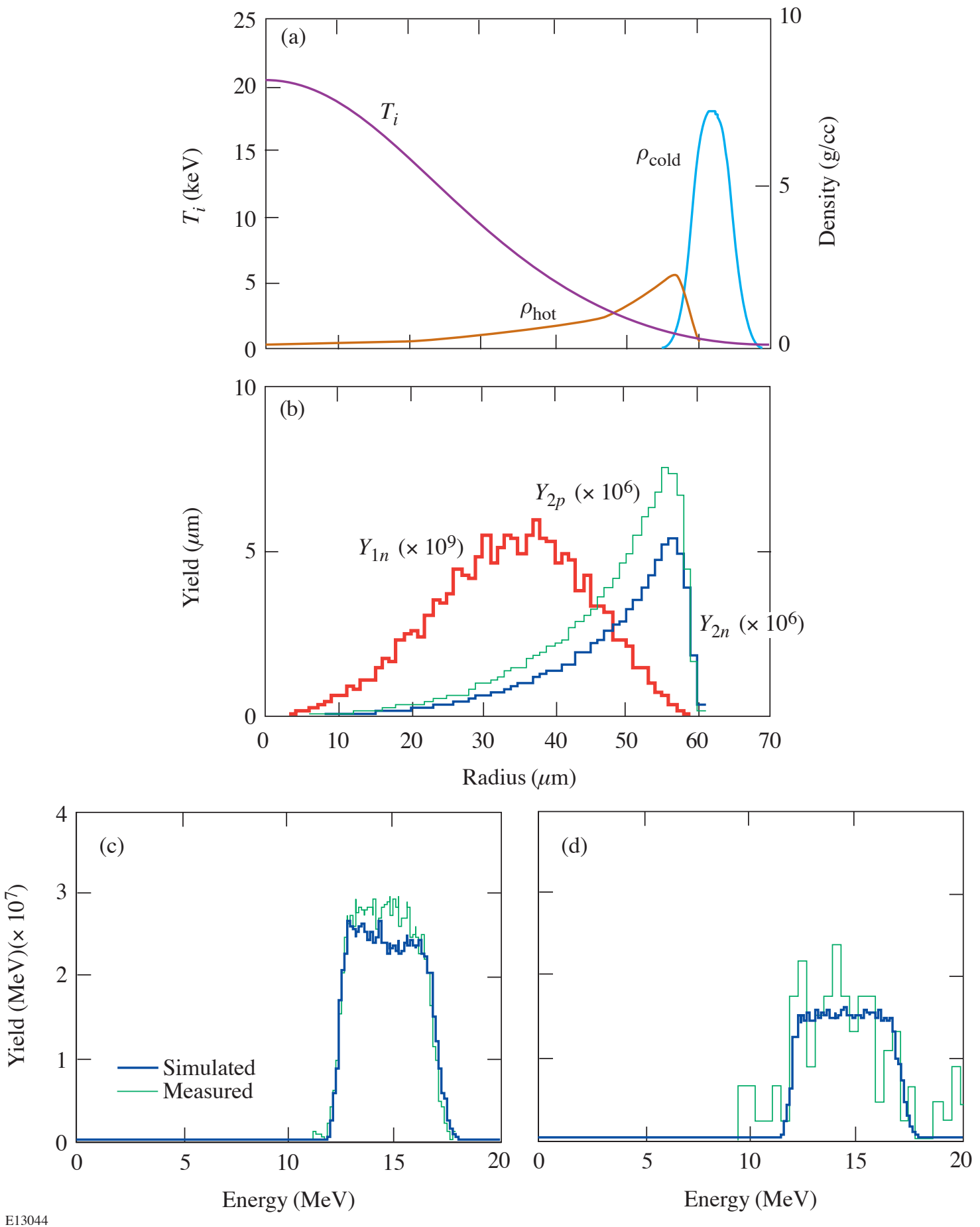

\section{Figure 98.56}

Parameters from the best-fit Monte Carlo simulation of shot $30981\left(3.1-\mu \mathrm{m} \mathrm{SiO}{ }_{2}\right.$ shell filled with 14.7 atm of $\left.\mathrm{D}_{2}\right)$. (a) $T_{i}(r)$ and $\rho(R)$. Fuel mass is fully conserved, while $11 \%$ of the shell mass remains. (b) Radial distributions of the birth positions of primary and secondary particles indicate that secondary protons and neutrons are produced in a virtually identical region of the capsule. (c) Measured and simulated secondary-proton and (d) secondary-neutron spectra. Note that the shape and width of the simulated proton spectrum are very similar to those of the measured spectrum, even though these were not part of the fitting procedure. The difference in simulated and measured secondary yields is within the measurement uncertainties. Measured and simulated values of implosion characteristics are summarized in Table 98.IV. Figure 98.60 indicates how the radial profiles of $T_{i}$ and $\rho$ can change without changing too much the quality of the fit to the data. 
Table 98.IV: Measured and simulated values of yields and $\rho R$ for OMEGA implosion 30981. Experimental data were fitted by adjusting $\rho(R)$ and $T_{i}(r)$. Total $\chi^{2}$ along with parameters specifying the cold $\left(\mathrm{SiO}_{2}\right)$ temperature and density Gaussian profiles [peak temperature $\left(T_{i 0}\right), 1 / e$ radius $\left(T_{i w}\right)$, power of the exponent $\left(T_{i p}\right)$, peak density radius $\left(S_{r 0}\right), 1 / \mathrm{e}$ radius $\left(S_{w}\right)$, and power of the exponent $\left.\left(S_{p}\right)\right]$ are also listed. $\rho R_{\text {cold }}=\int \rho_{\text {cold }} d r$, integrated radially over the $\mathrm{SiO}_{2}$ shell region, and $\rho R_{\text {hot }}=\int \rho_{\mathrm{D}} d r$, integrated radially over the hot-fuel region of the simulated profiles. Values of $\rho R_{\text {hot }, 2 n}$ and $\rho R_{\text {hot }, 2 p}$ were deduced using measured and simulated yield ratios assuming a $1.5 \pm 1-\mathrm{g} / \mathrm{cc}$ [obtained from Fig. 98.56(a)] D plasma at $\left\langle T_{i}\right\rangle_{Y_{1 n}} \pm 0.5 \mathrm{keV}$.

\begin{tabular}{|c|c|c|}
\hline \multicolumn{3}{|c|}{ Shot 30981} \\
\hline & Measured & Simulated \\
\hline$Y_{1 n}$ & $(1.5 \pm 0.15) \times 10^{11}$ & $(1.5+0.23-0.18) \times 10^{11}$ \\
\hline$Y_{2 n} / Y_{1 n}$ & $(5.1 \pm 0.98) \times 10^{-4}$ & $(5.1+1.1-0.57) \times 10^{-4}$ \\
\hline$Y_{2 p} / Y_{1 n}$ & $(7.9 \pm 1.1) \times 10^{-4}$ & $(7.6+1.0-0.96) \times 10^{-4}$ \\
\hline$\left\langle E_{2 p}\right\rangle(\mathrm{MeV})$ & $14.47 \pm 0.1$ & $14.64+0.14-0.16$ \\
\hline$\left\langle T_{i}\right\rangle_{Y_{1 n}}(\mathrm{keV})$ & $8.2 \pm 0.5$ & $8.2+0.7-0.5$ \\
\hline$\chi^{2}$ & $\ldots$ & 0.1 \\
\hline$T_{i 0}(\mathrm{keV})$ & $\ldots$ & $20.5+2.5-10$ \\
\hline$T_{i w}(\mu \mathrm{m})$ & $\ldots$ & $34+14-4$ \\
\hline$T_{i p}$ & $\ldots$ & $2+5-0$ \\
\hline$S_{r 0}(\mu \mathrm{m})$ & $\cdots$ & $62+6-10$ \\
\hline$S_{w}(\mu \mathrm{m})$ & $\ldots$ & $3.5+3-3.3$ \\
\hline$S_{p}$ & $\ldots$ & $2.5+7.5-2$ \\
\hline$\rho R_{\text {cold }}\left(\mathrm{mg} / \mathrm{cm}^{2}\right)$ & $\ldots$ & $4.5+4.3-4.2$ \\
\hline$\rho R_{\text {hot }}\left(\mathrm{mg} / \mathrm{cm}^{2}\right)$ & $\ldots$ & $3.7+0.8-0.4$ \\
\hline$\rho R_{\mathrm{hot}, 2 n}\left(\mathrm{mg} / \mathrm{cm}^{2}\right)$ & $4.6+0.9-1.2$ & $4.6+1.0-0.6$ \\
\hline$\rho R_{\mathrm{hot}, 2 p}\left(\mathrm{mg} / \mathrm{cm}^{2}\right)$ & $4.3+0.6-0.8$ & $4.1 \pm 0.5$ \\
\hline
\end{tabular}

\section{Cryogenic Implosions}

For cryogenic implosions, the interpretation of inferred values of $\rho R_{\text {hot }}$ is even more subtle since there is a hightemperature, low-density fuel region and a low-temperature, high-density fuel region. If most of the secondary particles are produced only in the hot-fuel region, $Y_{2} / Y_{1 n}$ can be used to infer $\rho R_{\text {hot }}$. On the other hand, if secondary particles are produced mainly in the inner part of the cold-fuel region, the inferred $\rho R$ is larger than $\rho R_{\text {hot }}$, but smaller than $\rho R_{\text {total }}$. (Even the more-penetrating $\mathrm{T}$ cannot traverse the entire cold-fuel region since the range of $\mathrm{T}$ in an $8 \mathrm{-g} / \mathrm{cc}, 1-\mathrm{keV} \mathrm{D}$ plasma is $\sim 15 \mathrm{mg} / \mathrm{cm}^{2}$, and we usually calculate $\rho R_{\text {total }}>40 \mathrm{mg} / \mathrm{cm}^{2}$ from the downshift of the average secondary-proton energy for cryogenic implosions.) Figure 98.51 shows that values of $\rho R_{\text {hot }}$ implied by measured $Y_{2 n} / Y_{1 n}$ are always larger than values from measured $Y_{2 p} / Y_{1 n}$ for those implosions (values were inferred assuming a $\left\langle T_{i}\right\rangle_{Y_{1 n}} \mathrm{keV}, 3$-g/cc D plasma). 

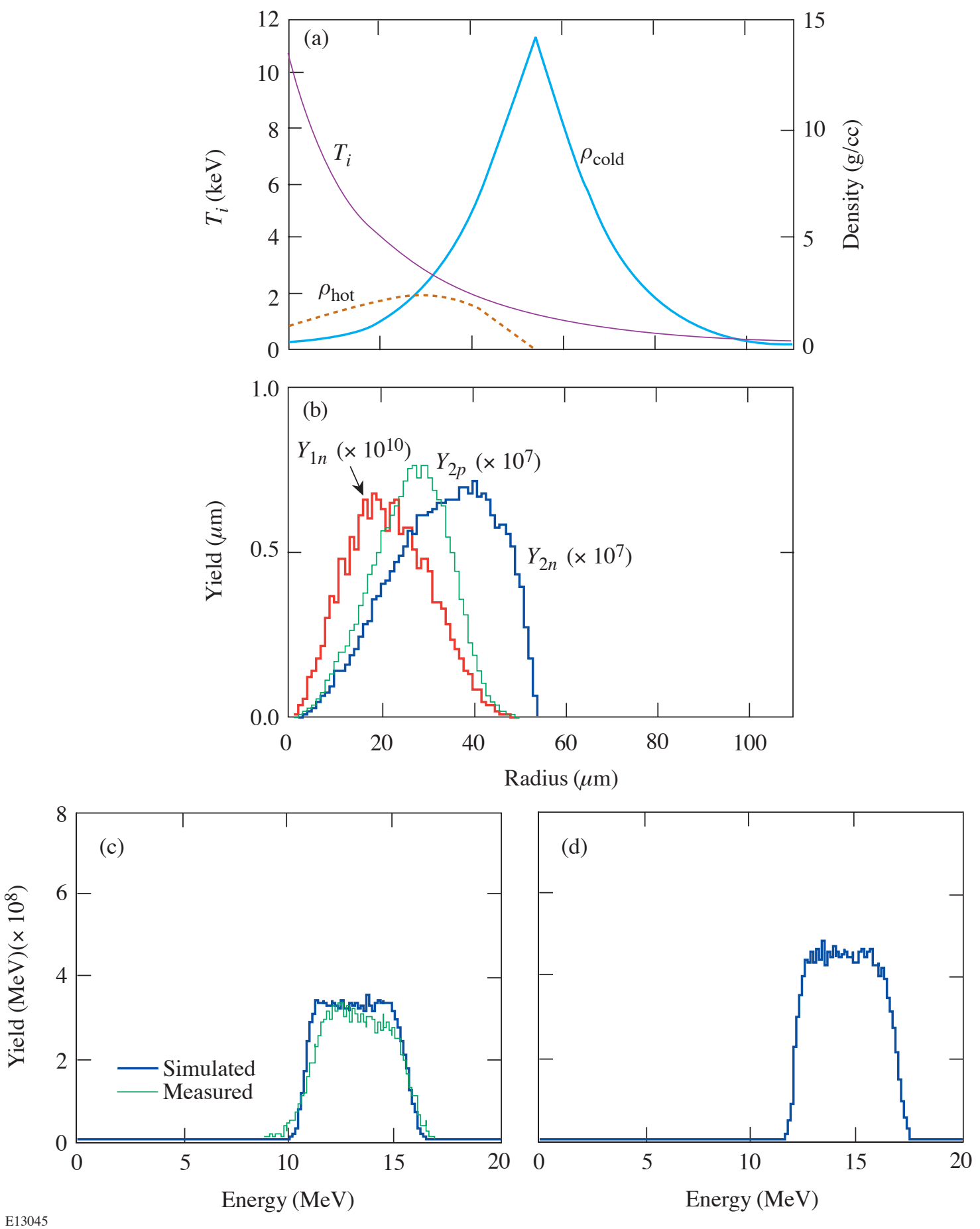

Figure 98.57

Best-fit parameters from the Monte Carlo simulation for shot 27443, which involved a 19.4- $\mu$ m CH shell filled with 15 atm of $\mathrm{D}_{2}$. (a) $T_{i}(r)$ and $\rho(r)$. Fuel mass is fully conserved, while $32 \%$ of the shell mass remains. (b) Radial distributions of the birth positions of primary and secondary particles show that secondaryproton production is diminished, while secondary-neutron production is enhanced in the region of significant fuel-shell mix. This causes secondary protons to underestimate and secondary neutrons to overestimate the actual value of $\rho R_{\text {hot }}$ (c) Measured and simulated secondary-proton spectra are compared, and (d) simulated secondary-neutron spectrum is shown. The secondary-proton spectra show more energy downshift, and the width of the secondary spectra are slightly narrower than the low $\rho R_{\text {hot }}$ case because the average primary particle energy is smaller at the time of secondary reaction. Measured and simulated values of implosion characteristics are listed in Table 98.V, while other fits are illustrated in Fig. 98.61. 
Table 98.V: Measured and simulated values of implosion characteristics for OMEGA implosion 27443. Values $\rho R_{\text {hot }}$ were calculated assuming a $2 \pm 1$-g/cc D plasma at $\left\langle T_{i}\right\rangle_{Y_{1 n}} \pm 0.5 \mathrm{keV}$. Results from simulation indicate that the $\rho R_{\mathrm{hot}, 2 p}$ underestimates and $\rho R_{\mathrm{hot}, 2 n}$ overestimates the actual value.

\begin{tabular}{|l|c|c|}
\hline & Measured & Simulated \\
\hline$Y_{1 n}$ & $(1.5 \pm 0.15) \times 10^{11}$ & $(1.6+0.1-0.25) \times 10^{11}$ \\
\hline$Y_{2 n} / Y_{1 n}$ & $(1.5 \pm 0.24) \times 10^{-3}$ & $(1.4+0.16-0.12) \times 10^{-3}$ \\
\hline$Y_{2 p} / Y_{1 n}$ & $(1.0 \pm 0.14) \times 10^{-3}$ & $(1.0+0.1-0.15) \times 10^{-3}$ \\
\hline$\left\langle E_{2 p}\right\rangle(\mathrm{MeV})$ & $13.1 \pm 0.1$ & $13.07+0.1-0.11$ \\
\hline$\left\langle T_{i}\right\rangle_{Y_{1 n}}(\mathrm{keV})$ & $4.1 \pm 0.5$ & $4.1+0.2-0.4$ \\
\hline & & \\
\hline$\chi^{2}$ & $\ldots$ & 0.5 \\
\hline$T_{i 0}(\mathrm{keV})$ & $\ldots$ & $11+0-5.5$ \\
\hline$T_{i w}(\mu \mathrm{m})$ & $\ldots$ & $0.8+1.2-0$ \\
\hline$T_{i p}$ & $\ldots$ & $54 \pm 2$ \\
\hline$S_{r 0}(\mu \mathrm{m})$ & $\ldots$ & $16+2-6$ \\
\hline$S_{w}(\mu \mathrm{m})$ & $\ldots$ & $1.2+0-0.2$ \\
\hline$S_{p}$ & $\ldots .8 \pm 1.9$ & $5.2+0.5-0.7$ \\
\hline & & \\
\hline$\rho R_{\mathrm{cold}}\left(\mathrm{mg} / \mathrm{cm}^{2}\right)$ & $\ldots .0 \pm 0.7$ & $42.3+3.9-2.1$ \\
\hline$\rho R_{\mathrm{hot}}\left(\mathrm{mg} / \mathrm{cm}^{2}\right)$ & & $8.9+1-0.4$ \\
\hline & & \\
\hline$\rho R_{\mathrm{hot}, 2 n}\left(\mathrm{mg} / \mathrm{cm}^{2}\right)$ & $\ldots$ & $11.6+1.2-1$ \\
\hline$\rho R_{\mathrm{hot}, 2 p}\left(\mathrm{mg} / \mathrm{cm}^{2}\right)$ & $\ldots$ & \\
\hline \hline
\end{tabular}

Radial profiles of temperature and density calculated for implosion $28900(89-\mu \mathrm{m} \mathrm{D}$-ice layer inside a $5.1-\mu \mathrm{m} \mathrm{CH}$ shell) are shown in Fig. 98.58(a), and simulated and measured spectra are shown in Figs. 98.58(c) and 98.58(d). As indicated in Fig. 98.58(d) and Fig. 98.59, the secondary-neutron spectrum is much narrower than the secondary-neutron spectra from Figs. 98.56(d) and 98.57(d) because the primary $\mathrm{T}$ are, on average, less energetic when they fuse with thermal D. ${ }^{12}$ Measurements of secondary-neutron spectra from morerecent cryogenic implosions also show the same characteristics.
The radial distributions of the primary- and secondary-birth positions shown in Fig. 98.58(b) indicate that secondary protons and neutrons are born mainly in the hot- and cold-fuel regions, respectively. Therefore, the $\rho R$ obtained from secondary protons gives an estimate of $\rho R_{\text {hot }}$, while the secondaryneutron yield provides a lower limit on $\rho R_{\text {total }}$. In this type of implosion, effects of mix or exchange of hot and cold fuel play significant roles in determining the radial distribution of secondary-birth positions. 

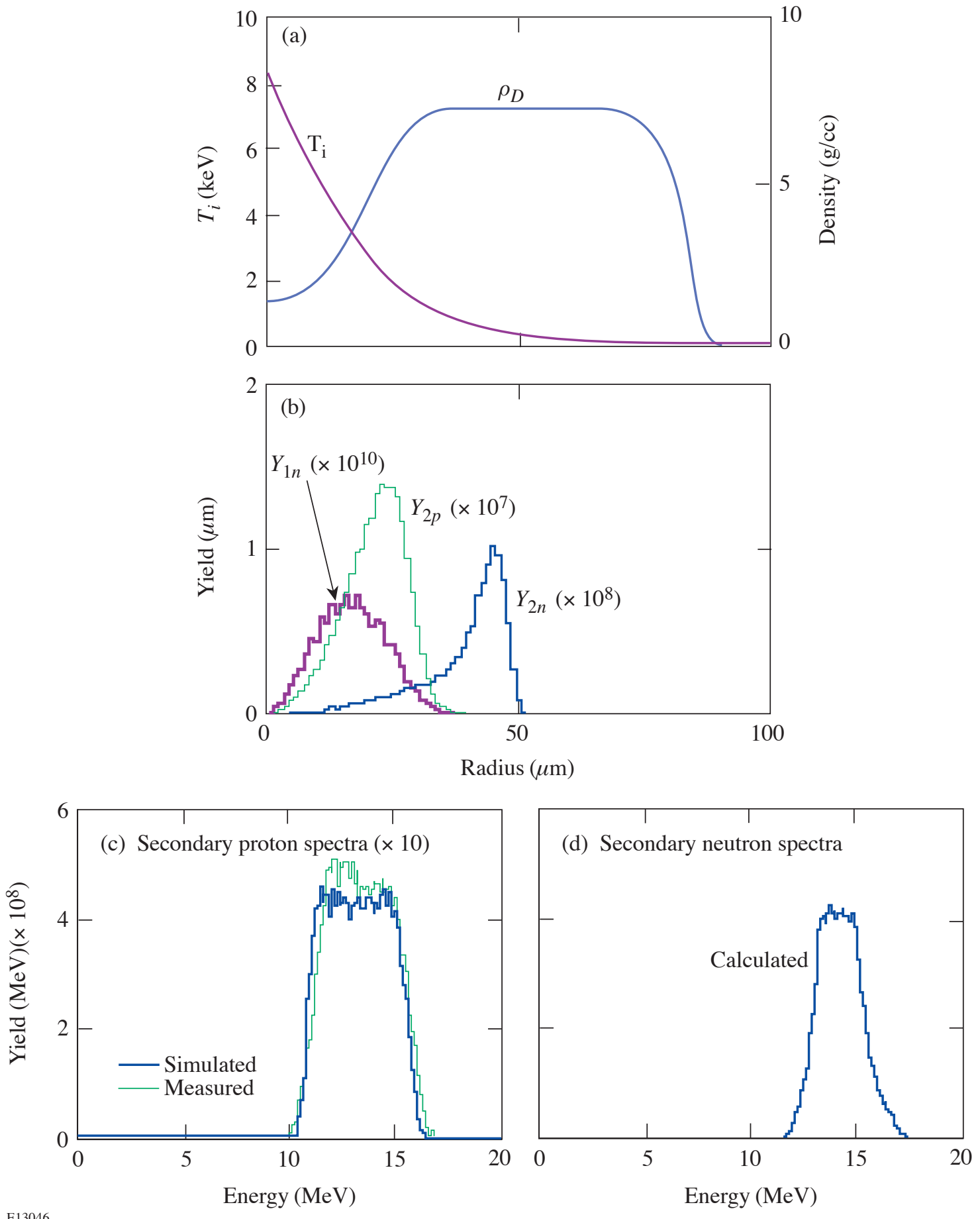

Figure 98.58

(a) Simulated profile of shot 28900 (cryogenic capsule with a $5.1-\mu \mathrm{m} \mathrm{CD}$ shell and $89-\mu \mathrm{m} \mathrm{D}_{2}$-ice layer), which gives the best fit to the measurement; $31 \%$ of the total mass remains. (b) Radial distributions of the birth points of primary and secondary particles show that most of the secondary protons are produced in the hot-fuel region, while secondary neutrons are mainly produced in the cold-fuel region. (c) Measured and simulated secondary-proton spectra. (d) Simulated secondary neutron spectrum is narrower than the spectra in Figs. 98.56(d) and 98.57(d) because primary T are less energetic at the time they undergo secondary reactions; $\rho R$ of cold fuel is large enough to stop primary T [Fig. 98.58(b)], and the cross section increases as T loses energy [Fig. 98.53(a)]. Important implosion characteristics are summarized in Table 98.VI, while other fits are illustrated in Fig. 98.62. 


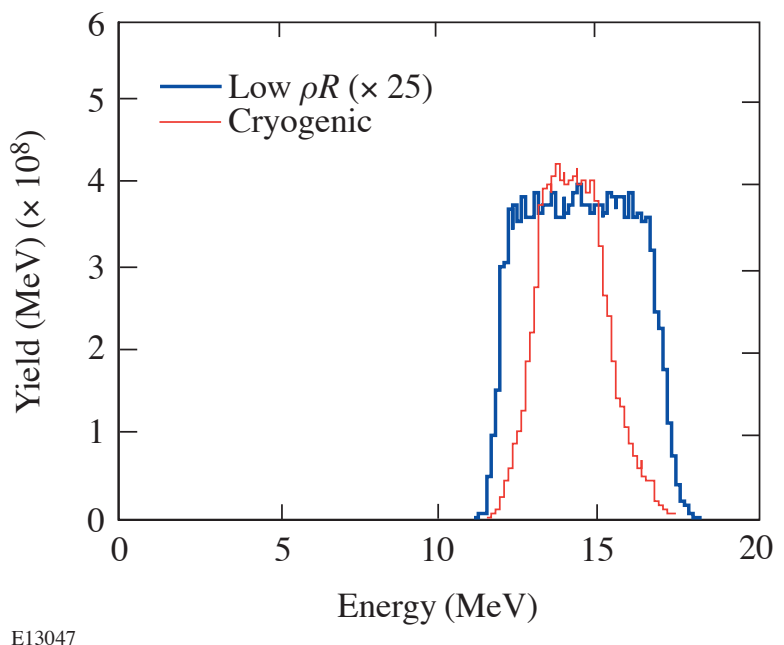

E13047

Simulated values of yields and other important implosion characteristics are compared with measured results in Table 98.VI. The secondary-neutron, hot-spot-model-inferred $\rho R^{\operatorname{sim} 1}$ is close to $\rho R_{\mathrm{total}}^{\text {int }}$, but this does not mean that the hot-spot model describes the implosion accurately. The agreement is an accidental consequence of using the wrong temperature $\left\langle T_{i}\right\rangle_{Y_{1 n}}$, which samples the hotter central region rather than the cooler fuel region where most of the secondary neutrons are produced.

This implosion has also been analyzed using a combination of x-ray and neutron measurements, without the use of secondary-proton data. These results are discussed in Ref. 37 . While the best-fit profiles were somewhat different, they agree within the uncertainties of the two simulation techniques.

\section{Conclusions}

The hot-spot and uniform models have been used to infer the areal density of the hot-fuel region $\left(\rho R_{\text {hot }}\right)$ of $\mathrm{D}_{2}$ implosions, but disagreements between the values of $\rho R_{\text {hot }}$ inferred from secondary-proton and secondary-neutron yields have often been observed, indicating limitations in these models. Results from direct-drive experiments on the OMEGA laser system and Monte Carlo simulations provided a deeper understanding of the relationship between $\rho R$, the capsule structure, and secondary-particle production. Experiments show that values of $\rho R_{\text {hot }}$ inferred from the ratios of secondary-proton and neutron-to-primary neutron yields ( $Y_{2 p} / Y_{1 n}$ and $Y_{2 n} / Y_{1 n}$ ) using the hot-spot model agree well for low- $\rho R_{\text {hot }}$ implosions (thin-glass-shell capsules), and simulations indicate that they
Figure 98.59

The simulated secondary-neutron spectrum is narrower than the spectra in Figs. 98.56(d) and 98.57(d) because the primary T are less energetic at the time they undergo secondary reactions; $\rho R$ of cold fuel is large enough to stop primary T [Fig. 98.58(b)], and the cross section increases as T loses energy [Fig. 98.53(a)]. Note that detailed analysis of secondary-neutron spectra was used to study areal density in Ref. 10. give a good estimate of the actual value of $\rho R_{\text {hot }}$. The results from implosions of $\mathrm{D}_{2}$-filled, thin-glass shells also show reasonably good agreement with results from implosions of similar capsules filled with DT gas. For thick-plastic-shellcapsule implosions, where the $\rho R_{\text {hot }}$ of an implosion becomes sufficiently large, $Y_{2 p} / Y_{1 n}$ underestimates $\rho R_{\text {hot }}$ since the primary ${ }^{3} \mathrm{He}$ are ranged out before sampling the entire hot-fuel region. In addition, fuel-shell mix increases the rate of energy loss of ${ }^{3} \mathrm{He}$ and causes $Y_{2 p} / Y_{1 n}$ to further underestimate $\rho R_{\text {hot }}$. The fuel-shell mix also causes $Y_{2 n} / Y_{1 n}$ to overestimate $\rho R_{\text {hot }}$ by slowing down the primary $\mathrm{T}$, thereby increasing the secondary DT fusion reaction cross section. As a result, values of $\rho R_{\text {hot }}$ for medium $\rho R_{\text {hot }}$ capsules inferred from $Y_{2 p} / Y_{1 n}$ and $Y_{2 n} / Y_{1 n}$ using the hot-spot model should be interpreted as estimates of the lower and upper limits on the actual $\rho R_{\text {hot }}$, respectively. For cryogenic capsules, secondary protons are produced mainly in the hot-fuel region, and the proton-implied value of $\rho R$ provides a good estimate of the hot-fuel $\rho R$. In contrast, secondary neutrons are mostly produced in the inner part of the cold-fuel region, and the neutron-implied $\rho R$ gives a lower limit on the total $\rho R$ when calculated correctly using the average temperature and density of the secondary-neutron birth point. Naive use of the simple hot-spot or uniform model, with a burn-averaged temperature, often results in inaccurate inference of $\rho R_{\text {hot }}$. A more-thorough analysis, such as the use of complete data sets and simulations to determine the secondary-birth positions and the effects of mix, as presented herein, or the use of detailed analysis of secondary-neutron spectra both from experiments and simulations, ${ }^{10}$ is required in order to obtain a realistic estimate of $\rho R_{\text {hot }}$. 
Table 98.VI: $\quad$ Measured and simulated values of implosion characteristics for OMEGA implosion 28900. $\rho R_{\text {total }}=\int \rho_{\mathrm{D}} d r$, integrated radially over the entire simulated profiles; $\rho R_{\text {hot }}$ is defined as the $\rho R$ that includes $90 \%$ of primary production. Values of $\rho R_{\text {hot }}$ were calculated assuming a $3.0 \pm 1.5$-g/cc D plasma at $\left\langle T_{i}\right\rangle_{Y_{1 n}} \pm 0.5 \mathrm{keV}$. Results from the simulation suggest that the value of $\rho R_{\text {hot }, 2 p}$ provides a good estimate of $\rho R_{\text {hot }}$. Secondary-neutron implied $\rho R_{\text {hot }}$ is similar to $\rho R_{\text {total }}$, but this is because the value of the temperature used to infer $\rho R_{\text {hot }}$ is too large. If the temperature of the cold-fuel region ( $1 \mathrm{keV}$ instead of $3.6 \mathrm{keV}$ ) were used, a much smaller and physical value of $\rho R_{\text {hot }}$ would be implied.

\begin{tabular}{|c|c|c|}
\hline \multicolumn{3}{|c|}{ Shot 28900} \\
\hline & Measured & Simulated \\
\hline$Y_{1 n}$ & $(1.2 \pm 0.12) \times 10^{11}$ & $(1.3+0.12-0.14) \times 10^{11}$ \\
\hline$Y_{2 n} / Y_{1 n}$ & $(9.4 \pm 1.4) \times 10^{-3}$ & $(9.1+1.0-1.1) \times 10^{-3}$ \\
\hline$Y_{2 p} / Y_{1 n}$ & $(1.8 \pm 0.26) \times 10^{-3}$ & $(1.6+0.0-0.2) \times 10^{-3}$ \\
\hline$\left\langle E_{2 p}\right\rangle(\mathrm{MeV})$ & $13.31 \pm 0.10$ & $13.28+0.5-0.11$ \\
\hline$\left\langle T_{i}\right\rangle_{Y_{1 n}}(\mathrm{keV})$ & $3.6 \pm 0.5$ & $3.5+0.6-0.3$ \\
\hline$\chi^{2}$ & $\cdots$ & 0.6 \\
\hline$T_{i 0}(\mathrm{keV})$ & $\ldots$ & $8.5+9.5-2.5$ \\
\hline$T_{i w}(\mu \mathrm{m})$ & $\ldots$ & $18+10-8$ \\
\hline$T_{i p}$ & $\cdots$ & $1.2+0.6-0.4$ \\
\hline$S_{r 0}(\mu \mathrm{m})$ & $\ldots$ & $52+22-2$ \\
\hline$S_{w}(\mu \mathrm{m})$ & $\ldots$ & $32+16-12$ \\
\hline$S_{p}$ & $\cdots$ & $9+\geq 1-7.5$ \\
\hline$\rho R_{\text {total }}\left(\mathrm{mg} / \mathrm{cm}^{2}\right)$ & $\cdots$ & $48.2+3.2-6.0$ \\
\hline$\rho R_{\mathrm{hot}}\left(\mathrm{mg} / \mathrm{cm}^{2}\right)$ & $\cdots$ & $7.9+0.2-1.7$ \\
\hline$\rho R_{\mathrm{hot}, 2 n}\left(\mathrm{mg} / \mathrm{cm}^{2}\right)$ & $49.8+5.0-6.9$ & $48.0+4.9-4.0$ \\
\hline$\rho R_{\mathrm{hot}, 2 p}\left(\mathrm{mg} / \mathrm{cm}^{2}\right)$ & $9.3+1.9-1.5$ & $7.8+0.5-0.6$ \\
\hline
\end{tabular}



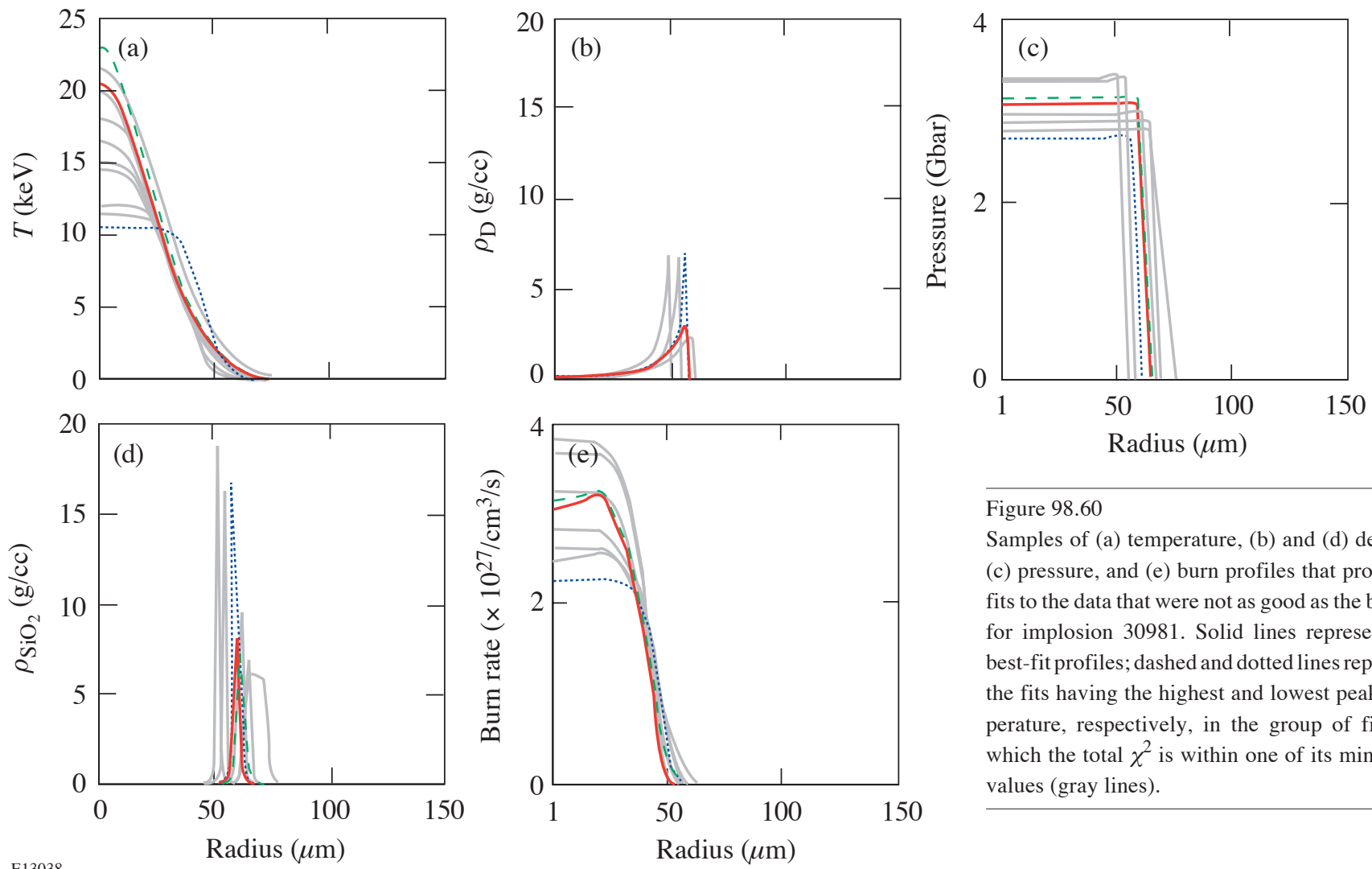

Figure 98.60

Samples of (a) temperature, (b) and (d) density, (c) pressure, and (e) burn profiles that produced fits to the data that were not as good as the best fit for implosion 30981. Solid lines represent the best-fit profiles; dashed and dotted lines represent the fits having the highest and lowest peak temperature, respectively, in the group of fits for which the total $\chi^{2}$ is within one of its minimum values (gray lines).

E13038
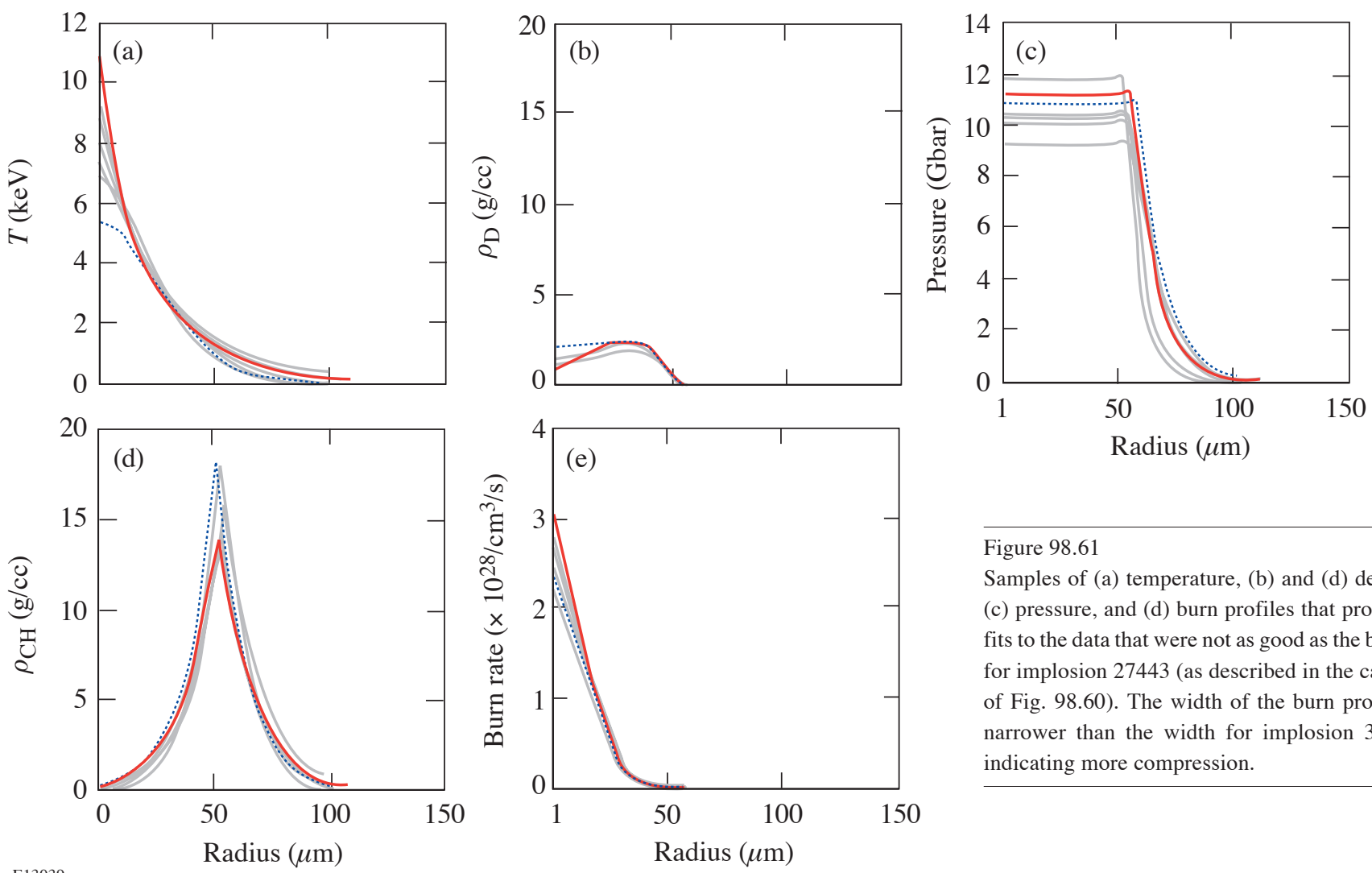

Figure 98.61

Samples of (a) temperature, (b) and (d) density, (c) pressure, and (d) burn profiles that produced fits to the data that were not as good as the best fit for implosion 27443 (as described in the caption of Fig. 98.60). The width of the burn profile is narrower than the width for implosion 30981, indicating more compression.

E13039

Radius $(\mu \mathrm{m})$ 
Using Nuclear Data and Monte Carlo Techniques to Study Areal Density and Mix in $D_{2}$ Implosions
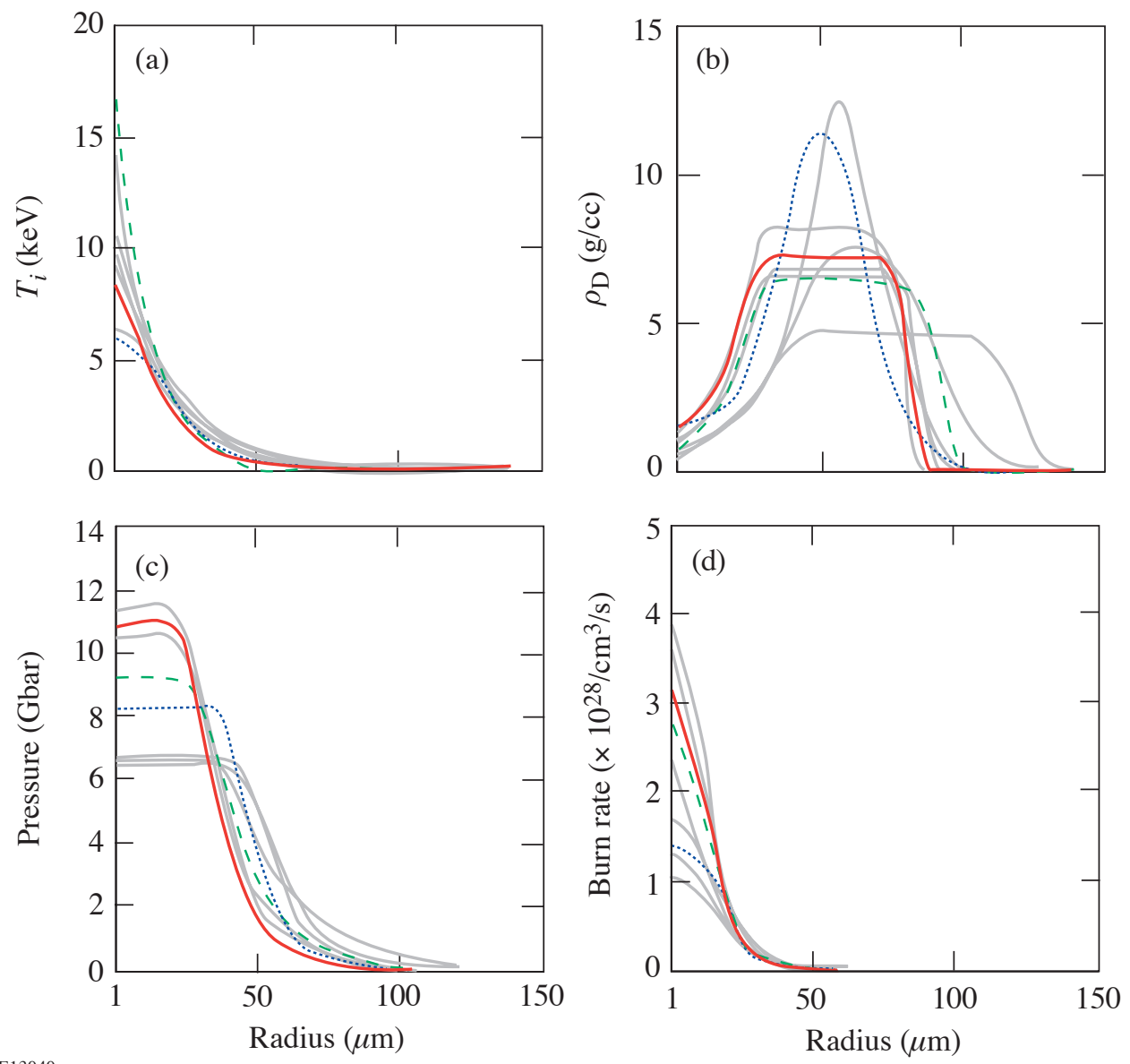

Figure 98.62

Samples of (a) temperature, (b) density, (c) pressure, and (d) burn profiles that produced fits to the data that were not as good as the best fit for implosion 28900 (as described in the caption of Fig. 98.60). The width of the burn profile is narrower than the width for implosion 30981, indicating more compression. 


\section{ACKNOWLEDGMENT}

The work was performed in part at LLE's National Laser Users' Facility (NLUF) and was supported in part by the U.S. DOE (grant DE-FG0303SF22691), US DOE DP/NNSA (Cooperative Agreement DE-FG0303NA00058), LLE (subcontract 412160-001G), and LLNL (subcontract B504974).

\section{REFERENCES}

1. S. W. Haan et al., Phys. Plasmas 2, 2480 (1995).

2. J. D. Lindl, R. L. McCrory, and E. M. Campbell, Phys. Today 45, 32 (1992).

3. J. D. Lindl, Phys. Plasmas 2, 3933 (1995).

4. H. D. Campbell and F. H. Southworth, in 1st Topical Meeting on the Technology of Controlled Nuclear Fusion (American Nuclear Society, Hinsdale, IL, 1974), pp. 75-76.

5. E. G. Gamalii et al., JETP Lett. 21, 70 (1975).

6. S. Skupsky and S. Kacenjar, J. Appl. Phys. 52, 2608 (1981).

7. T. E. Blue and D. B. Harris, Nucl. Sci. Eng. 77, 463 (1981).

8. T. E. Blue et al., J. Appl. Phys. 54, 615 (1983).

9. H. Azechi, M. D. Cable, and R. O. Stapf, Laser Part. Beams 9, 119 (1991).

10. M. D. Cable and S. P. Hatchett, J. Appl. Phys. 62, 2233 (1987).

11. H. Azechi et al., Appl. Phys. Lett. 49, 555 (1986).

12. F. H. Séguin, C. K. Li, D. G. Hicks, J. A. Frenje, K. M. Green, R. D. Petrasso, J. M. Soures, D. D. Meyerhofer, V. Yu. Glebov, C. Stoeckl, P. B. Radha, S. Roberts, C. Sorce, T. C. Sangster, M. D. Cable, S. Padalino, and K. Fletcher, Phys. Plasmas 9, 2725 (2002).

13. P. Amendt, R. E. Turner, and O. L. Landen, Phys. Rev. Lett. 89, 165001 (2002).

14. T. C. Sangster, J. A. Delettrez, R. Epstein, V. Yu. Glebov, V. N. Goncharov, D. R. Harding, J. P. Knauer, R. L. Keck, J. D. Kilkenny, S. J. Loucks, L. D. Lund, R. L. McCrory, P. W. McKenty, F. J. Marshall, D. D. Meyerhofer, S. F. B. Morse, S. P. Regan, P. B. Radha, S. Roberts, W. Seka, S. Skupsky, V. A. Smalyuk, C. Sorce, J. M. Soures, C. Stoeckl, K. Thorp, J. A. Frenje, C. K. Li, R. D. Petrasso, F. H. Séguin, K. A. Fletcher, S. Padalino, C. Freeman, N. Izumi, J. A. Koch, R. A. Lerche, M. J. Moran, T. W. Phillips, and G. J. Schmid, Phys. Plasmas 10, 1937 (2003).

15. C. Stoeckl, C. Chiritescu, J. A. Delettrez, R. Epstein, V. Yu. Glebov, D. R. Harding, R. L. Keck, S. J. Loucks, L. D. Lund, R. L. McCrory, P. W. McKenty, F. J. Marshall, D. D. Meyerhofer, S. F. B. Morse, S. P. Regan, P. B. Radha, S. Roberts, T. C. Sangster, W. Seka, S. Skupsky, V. A. Smalyuk, C. Sorce, J. M. Soures, R. P. J. Town, J. A. Frenje, C. K. Li, R. D. Petrasso, F. H. Séguin, K. Fletcher, S. Padalino, C. Freeman, N. Izumi, R. Lerche, and T. W. Phillips, Phys. Plasmas 9, 2195 (2002).
16. R. E. Turner, P. A. Amendt, O. L. Landen, L. J. Suter, R. J. Wallace, H. E. Dalhed, B. A. Hammel, and V. Glebov, Bull. Am. Phys. Soc. 47, 328 (2002).

17. T. R. Boehly, D. L. Brown, R. S. Craxton, R. L. Keck, J. P. Knauer, J. H. Kelly, T. J. Kessler, S. A. Kumpan, S. J. Loucks, S. A. Letzring, F. J. Marshall, R. L. McCrory, S. F. B. Morse, W. Seka, J. M. Soures, and C. P. Verdon, Opt. Commun. 133, 495 (1997).

18. S. Glasstone and R. H. Lovberg, Controlled Thermonuclear Reactions: An Introduction to Theory and Experiment (Van Nostrand, Princeton, NJ, 1960).

19. C. K. Li and R. D. Petrasso, Phys. Rev. Lett. 70, 3059 (1993).

20. P. B. Radha, J. Delettrez, R. Epstein, V. Yu. Glebov, R. Keck, R. L. McCrory, P. McKenty, D. D. Meyerhofer, F. Marshall, S. P. Regan, S. Roberts, T. C. Sangster, W. Seka, S. Skupsky, V. Smalyuk, C. Sorce, C. Stoeckl, J. Soures, R. P. J. Town, B. Yaakobi, J. Frenje, C. K. Li, R. Petrasso, F. Séguin, K. Fletcher, S. Padalino, C. Freeman, N. Izumi, R. Lerche, and T. W. Phillips, Phys. Plasmas 9, 2208 (2002).

21. C. K. Li, F. H. Séguin, J. A. Frenje, S. Kurebayashi, R. D. Petrasso, D. D. Meyerhofer, J. M. Soures, J. A. Delettrez, V. Yu. Glebov, P. B. Radha, F. J. Marshall, S. P. Regan, S. Roberts, T. C. Sangster, and C. Stoeckl, Phys. Rev. Lett. 89, 165002 (2002).

22. S. P. Regan, J. A. Delettrez, F. J. Marshall, J. M. Soures, V. A. Smalyuk, B. Yaakobi, V. Yu. Glebov, P. A. Jaanimagi, D. D. Meyerhofer, P. B. Radha, W. Seka, S. Skupsky, C. Stoeckl, R. P. J. Town, D. A. Haynes, Jr., I. E. Golovkin, C. F. Hooper, Jr., J. A. Frenje, C. K. Li, R. D. Petrasso, and F. H. Séguin, Phys. Rev. Lett. 89, 085003 (2002).

23. Y. Lin, T. J. Kessler, and G. N. Lawrence, Opt. Lett. 20, 764 (1995).

24. T. R. Boehly, V. A. Smalyuk, D. D. Meyerhofer, J. P. Knauer, D. K. Bradley, R. S. Craxton, M. J. Guardalben, S. Skupsky, and T. J. Kessler, J. Appl. Phys. 85, 3444 (1999).

25. S. Skupsky, R. W. Short, T. Kessler, R. S. Craxton, S. Letzring, and J. M. Soures, J. Appl. Phys. 66, 3456 (1989).

26. Short laser pulses ( $<1 \mathrm{~ns})$ imploding thin-glass-shell capsules prevent significant nuclear production while the laser is on. This is important if $\rho R_{\text {total }}$ is to be studied because the capsule can be charged to a significant potential relative to the target chamber wall due to laserplasma interactions. The potential can cause an upshift of measured proton energies, $35,38,39$ making it difficult to determine how much energy the protons lost while leaving the capsule (necessary for calculating $\left.\rho R_{\text {total }}\right)$. The potential decays away rapidly after the laser is turned off, so it does not affect measurements of protons from implosions in which the nuclear burn occurs after the pulse (such as those involving thick-plastic-shell or cryogenic capsules ${ }^{35}$ ). This affects only the study of $\rho R_{\text {total }}$; measurements of yields are unaffected by capsule potentials.

27. F. H. Séguin, J. A. Frenje, C. K. Li, D. G. Hicks, S. Kurebayashi, J. R. Rygg, B.-E. Schwartz, R. D. Petrasso, S. Roberts, J. M. Soures, D. D. Meyerhofer, T. C. Sangster, J. P. Knauer, C. Sorce, V. Yu. Glebov, C. Stoeckl, T. W. Phillips, R. J. Leeper, K. Fletcher, and S. Padalino, Rev. Sci. Instrum. 74, 975 (2003). 
28. M. A. Russotto and R. L. Kremens, Rev. Sci. Instrum. 61, 3125 (1990).

29. R. A. Lerche, D. W. Phillion, and G. L. Tietbohl, Rev. Sci. Instrum. 66, 933 (1995)

30. V. Yu. Glebov, C. Stoeckl, S. Roberts, T. C. Sangster, J. A. Frenje, R. D. Petrasso, R. A. Lerche, and R. L. Griffith, Bull. Am. Phys. Soc. 48, 342 (2003).

31. Primary-neutron-yield-weighted average ion temperature is calculated by two methods, each yielding a virtually identical temperature. First, the product of the temperature and the primary neutron birth rate per unit length at each radius is integrated and divided by the primary neutron yield. Second, the primary neutron spectrum is calculated, and the width of the spectrum is used to obtain an ion temperature.

32. J. D. Lindl, Inertial Confinement Fusion: The Quest for Ignition and Energy Gain Using Indirect Drive (Springer-Verlag, New York, 1998).

33. M. D. Rosen and J. H. Nuckolls, Phys. Fluids 22, 1393 (1979).

34. M. J. Canavan, MIT, private communication (2004); herein the laser energy was $\sim 30 \mathrm{~kJ}$ for these DT implosions.
35. C. K. Li, F. H. Séguin, D. G. Hicks, J. A. Frenje, K. M. Green, S. Kurebayashi, R. D. Petrasso, D. D. Meyerhofer, J. M. Soures, V. Yu. Glebov, R. L. Keck, P. B. Radha, S. Roberts, W. Seka, S. Skupsky, C. Stoeckl, and T. C. Sangster, Phys. Plasmas 8, 4902 (2001).

36. P. B. Radha et al., ${ }^{20}$ C. K. Li et al., ${ }^{21}$ and S. P. Regan et al. ${ }^{22}$ reported $\sim 1 \mu \mathrm{m}, \sim 0.5 \mu \mathrm{m}$, and $\sim 0.4 \mu \mathrm{m}$ of the initial shell layer mixed into the fuel, respectively.

37. V. A. Smalyuk, J. A. Delettrez, S. B. Dumanis, R. Epstein, V. Yu. Glebov, D. D. Meyerhofer, P. B. Radha, T. C. Sangster, C. Stoeck1, N. C. Toscano, J. A. Frenje, C. K. Li, R. D. Petrasso, F. H. Séguin, and J. H. Koch, "Hot-Core Characterization of the Cryogenic $\mathrm{D}_{2}$ Target at Peak Neutron Production in Direct-Drive Spherical Implosion," submitted to Physical Review Letters.

38. D. G. Hicks, C. K. Li, F. H. Séguin, J. D. Schnittman, A. K. Ram, J. A. Frenje, R. D. Petrasso, J. M. Soures, D. D. Meyerhofer, S. Roberts, C. Sorce, C. Stoeckl, T. C. Sangster, and T. W. Phillips, Phys. Plasmas 8, 606 (2001).

39. D. G. Hicks, C. K. Li, F. H. Séguin, A. K. Ram, J. A. Frenje, R. D. Petrasso, J. M. Soures, V. Yu. Glebov, D. D. Meyerhofer, S. Roberts, C. Sorce, C. Stöckl, T. C. Sangster, and T. W. Phillips, Phys. Plasmas 7, 5106 (2000). 


\section{Publications and Conference Presentations}

E. L. Alfonso, R. Q. Gram, and D. R. Harding, "Modeling Temperature and Pressure Gradients During Cooling of ThinWalled Cryogenic Targets," Fusion Sci. Technol. 45, 218 (2004).

K. Anderson and R. Betti, "Laser-Induced Adiabat Shaping by Relaxation in Inertial Fusion Implosions," Phys. Plasmas 11, 5 (2004).

V. Bagnoud and J. D. Zuegel, "Independent Phase and Amplitude Control of a Laser Beam by Use of a Single-Phase-Only Spatial Light Modulator,” Opt. Lett. 29, 295 (2004).

C. Dorrer and D. N. Maywar, "RF Spectrum Analysis of Optical Signals Using Nonlinear Optics," J. Lightwave Technol. 22, 266 (2004).

D. F. Grosz, A. Agarwal, S. Banerjee, D. N. Maywar, and A. P. Küng, "All-Raman Ultralong-Haul Single-Wideband DWDM Transmission Systems with OADM Capability," J. Lightwave Technol. 22, 423 (2004).

L. Guazzotto, R. Betti, J. Manickam, and S. Kaye, "Numerical Study of Tokamak Equilibria with Arbitrary Flow," Phys. Plasmas 11, 604 (2004).

T. J. Kessler, J. Bunkenburg, H. Huang, A. Kozlov, and D. D. Meyerhofer, "Demonstration of Coherent Addition of Multiple Gratings for High-Energy Chirped-Pulse-Amplified Lasers," Opt. Lett. 29, 635 (2004).

A. K. Knight, F.-Y. Tsai, M. J. Bonino, and D. R. Harding, "Suitability of Different Polyimide Capsule Materials for Use as ICF Targets," Fusion Sci. Technol. 45, 187 (2004).
T. Kosteski, N. P. Kherani, P. Stradins, F. Gaspari, W. T. Shmayda, L. S. Sidhu, and S. Zukotynski, "Tritiated Amorphous Silicon Betavoltaic Devices," IEE Proc.-Circuits Devices Syst. 150, 274 (2003).

T. I. Lakoba, C. Dorrer, and D. N. Maywar, "PolarizationMode Dispersion of a Circulating Loop," J. Opt. Soc. Am. B 21, 243 (2004).

J. Li, Y. Xu, T. Y. Hsiang, and W. R. Donaldson, "Picosecond Response of Gallium-Nitride Metal-Semiconductor-Metal Photodetectors," Appl. Phys. Lett. 84, 2091 (2004).

J. R. Marciante and D. H. Raguin, "High-Efficiency, HighDispersion Diffraction Gratings Based on Total Internal Reflection," Opt. Lett. 29, 542 (2004).

F. J. Marshall, J. A. Delettrez, R. Epstein, R. Forties, R. L. Keck, J. H. Kelly, P. W. McKenty, S. P. Regan, and L. J. Waxer, "Direct-Drive-Implosion Experiments with Enhanced Fluence Balance on OMEGA," Phys. Plasmas 11, 251 (2004).

X. Teng and H. Yang, "Effects of Surfactants and Synthetic Conditions on the Sizes and Self-Assembly of Monodisperse Iron Oxide Nanoparticles," J. Mater. Chem. 14, 774 (2004).

B. Yaakobi, D. D. Meyerhofer, T. R. Boehly, J. J. Rehr, B. A. Remington, P. G. Allen, S. M. Pollaine, and R. C. Albers, "Extended X-Ray Absorption Fine Structure Measurements of Laser-Shocked V and Ti and Crystal Phase Transformation in Ti,” Phys. Rev. Lett. 92, 095504 (2004).

J. D. Zuegel and D. W. Jacobs-Perkins, "Efficient, HighFrequency Bulk Phase Modulator,"Appl. Opt. 43, 1946 (2004). 
A. C. A. Chen, S. W. Culligan, Y. Geng, K. P. Klubek, K. M. Vaeth, and C. W. Tang, "Polarized Organic Light-Emitting Diodes Through Energy Transfer Using Monodisperse Conjugated Oligomers," to be published in Advanced Materials.

T. J. B. Collins, J. P. Knauer, R. Betti, T. R. Boehly, J. A. Delettrez, V. N. Goncharov, D. D. Meyerhofer, P. W. McKenty, S. Skupsky, and R. P. J. Town, "Reduction of the Ablative Rayleigh-Taylor Growth Rate with Gaussian Picket Pulses," to be published in Physics of Plasmas.

J.A. Frenje, C. K. Li, F. H. Séguin. J. Deciantis, S. Kurebayashi, J. R. Rygg, R. D. Petrasso, J. Delettrez, V. Yu. Glebov, C. Stoeckl, F. J. Marshall, D. D. Meyerhofer, T. C. Sangster, V. A. Smalyuk, and J. M. Soures, "Measuring Shock-Bang Timing and $\rho R$ Evolution of $D^{3} \mathrm{He}$ Implosions at OMEGA," to be published in Physics of Plasmas (invited).

C. K. Li, F. H. Séguin, J. A. Frenje, R. D. Petrasso, J. A. Delettrez, R. L. Keck, J. M. Soures, P. W. McKenty, F. J. Marshall, V. N. Goncharov, J. P. Knauer, D. D. Meyerhofer, P. B. Radha, S. P. Regan, T. C. Sangster, and W. Seka, “ $\rho R$ Asymmetry in the Spherical Implosions of Inertial Confinement Fusion," to be published in Physical Review Letters.

C. K. Li, F. H. Séguin, J. A. Frenje, R. D. Petrasso, J. A. Delettrez, P. W. McKenty, T. C. Sangster, R. L. Keck, J. M. Soures, F. J. Marshall, D. D. Meyerhofer, V. N. Goncharov, J. P. Knauer, P. B. Radha, S. P. Regan, and W. Seka, "Effects of Nonuniform Illumination on Implosion Asymmetry in DirectDrive Inertial Confinement Fusion," to be published in Physical Review Letters.

S. G. Lukishova, A. W. Schmid, C. Supranowitz, A. McNamara, R. W. Boyd, and C. R. Stroud, Jr., "Dye-Doped CholestericLiquid-Crystal Room-Temperature Single Photon Source," to be published in the Journal of Modern Optics.

A. V. Maximov, J. Myatt, W. Seka, R. W. Short, and R. S. Craxton, "Modeling of Stimulated Brillouin Scattering Near the Critical Density Surface in the Plasmas of Direct-Drive Targets," to be published in Physics of Plasmas.
R. L. McCrory, D. D. Meyerhofer, R. Betti, T. R. Boehly, R. S. Craxton, T. J. B. Collins, J. A. Delettrez, R. Epstein, V. Yu. Glebov, V. N. Goncharov, D. R. Harding, R. L. Keck, J. H. Kelly, J. P. Knauer, S. J. Loucks, L. Lund, J. A. Marozas, P. W. McKenty, F. J. Marshall, S. F. B. Morse, P. B. Radha, S. P. Regan, S. Roberts, W. Seka, S. Skupsky, V. A. Smalyuk, C. Sorce, C. Stoeckl, J. M. Soures, R. P. J. Town, B. Yaakobi, J. A. Frenje, C. K. Li, R. D. Petrasso, F. H. Séguin, K. A. Fletcher, S. Padalino, C. Freeman, and T. C. Sangster, "DirectDrive Inertial Confinement Fusion Research at the Laboratory for Laser Energetics," to be published in the proceedings of Current Trends in International Fusion Research: A Review.

R. L. McCrory, D. D. Meyerhofer, S. J. Loucks, S. Skupsky, R. E. Bahr, R. Betti, T. R. Boehly, R. S. Craxton, T. J. B. Collins, J. A. Delettrez, W. R. Donaldson, R. Epstein, J. A. Frenje, V. Yu. Glebov, V. N. Goncharov, D. R. Harding, P. A. Jaanimagi, R. L. Keck, J. H. Kelly, T. J. Kessler, J. P. Knauer, C. K. Li, L. D. Lund, J. A. Marozas, P. W. McKenty, F. J. Marshall, S. F. B. Morse, R. D. Petrasso, P. B. Radha, S. P. Regan, S. Roberts, T. C. Sangster, F. H. Séguin, W. Seka, V. A. Smalyuk, C. Sorce, J. M. Soures, C. Stoeckl, R. P. J. Town, B. Yaakobi, and J. D. Zuegel, "Progress in Direct-Drive Inertial Confinement Fusion Research at the Laboratory for Laser Energetics," to be published in Nuclear Fusion.

P. W. McKenty, T. C. Sangster, M. Alexander, R. Betti, R. S. Craxton, J. A. Delettrez, L. Elasky, R. Epstein, A. Frank, V. Yu. Glebov, V. N. Goncharov, D. R. Harding, S. Jin, J. P. Knauer, R. L. Keck, S. J. Loucks, L. D. Lund, R. L. McCrory, F. J. Marshall, D. D. Meyerhofer, S. P. Regan, P. B. Radha, S. Roberts, W. Seka, S. Skupsky, V. A. Smalyuk, J. M. Soures, K. A. Thorp, M. Wozniak, J. A. Frenje, C. K. Li, R. D. Petrasso, F. H. Séguin, K.A. Fletcher, S. Padalino, C. Freeman, N. Izumi, J. A. Koch, R. A. Lerche, M. J. Moran, T. W. Phillips, G. J. Schmid, and C. Sorce, "Direct-Drive Cryogenic Target Implosion Performance on OMEGA," to be published in Physics of Plasmas (invited).

J. Myatt, A. V. Maximov, W. Seka, R. S. Craxton, and R. W. Short, "Modeling SBS in the Underdense Corona of a DirectDrive Inertial Confinement Fusion Target," to be published in Physics of Plasmas. 
J.-R. Park, W. R. Donaldson, and R. Sobolewski, "Characterization of Single and Double Fiber Coupled Diffusing Spheres," to be published in Applied Optics.

S. P. Regan, J. A. Delettrez, V. N. Goncharov, F. J. Marshall, J. M. Soures, V. A. Smalyuk, P. B. Radha, B. Yaakobi, R. Epstein, V. Yu. Glebov, P. A. Jaanimagi, D. D. Meyerhofer, T. C. Sangster, W. Seka, S. Skupsky, C. Stoeckl, D. A. Haynes, Jr., J. A. Frenje, C. K. Li, R. D. Petrasso, and F. H. Séguin, "Dependence of Shell Mix on Feedthrough in Direct-Drive Inertial Confinement Fusion," to be published in Physical Review Letters.

B. A. Remington, G. Bazan, J. Belak, E. Bringa, M. Caturla, J. D. Colvin, M. J. Edwards, S. G. Glendinning, D. Ivanov, B. Kad, D. H. Kalantar, M. Kumar, B. F. Lasinski, K. T. Lorenz, J. M. McNaney, D. D. Meyerhofer, M. A. Meyers, S. M. Pollaine, D. Rowley, M. Schneider, J. S. Stölken, J. D. Wark, S. V. Weber, W. G. Wolfer, and B. Yaakobi, "Materials Science Under Extreme Conditions of Pressure and Strain Rate," to be published in Metallurgical and Materials Transactions A.
S. Skupsky, J. A. Marozas, R. S. Craxton, R. Betti, T. J. B. Collins, J. A. Delettrez, V. N. Goncharov, P. W. McKenty, P. B. Radha, T. R. Boehly, J. P. Knauer, F. J. Marshall, D. R. Harding, J. D. Kilkenny, D. D. Meyerhofer, T. C. Sangster, and R. L. McCrory, "Polar Direct Drive on the National Ignition Facility," to be published in Physics of Plasmas (invited).

A. Verevkin, A. Pearlman, W. Slysz, J. Zhang, R. Sobolewski, M. Currie, A. Korneev, G. Chulkova, O. Okunev, P. Kouminov, K. Smirnov, B. Voronov, and G. N. Gol'tsman, "Ultrafast Superconducting Single-Photon Detectors for Near-InfraredWavelength Quantum Communications," to be published in the Journal of Modern Optics.

B. Yaakobi, D. D. Meyerhofer, T. R. Boehly, J. J. Rehr, B. A. Remington, P. G. Allen, S. M. Pollaine, and R. C. Albers, "Extended X-Ray Absorption Fine Structure Measurements of Laser Shocks in Ti and V and Phase Transformation in Ti," to be published in Physics of Plasmas (invited).

\section{Conference Presentations}

J.-R. Park, W. R. Donaldson, and R. Sobolewski, "TimeResolved Imaging of a Spatially Modulated Laser Pulse," LASE 2004, San Jose, CA, 24-29 January 2004.

The following presentations were made at the 2004 Advanced Solid-State Photonics, Santa Fe, NM, 1-4 February 2004:

V. Bagnoud, J. Puth, and J. D. Zuegel, "High-Energy, 5-HzRepetition-Rate Laser Amplifier Using Wavefront-Corrected Nd:YLF Laser Rods.”

I. A. Begishev, V. Bagnoud, M. J. Guardalben, J. Puth, L. J. Waxer, and J. D. Zuegel, "Parasitic Second-Harmonic Generation in Optical Parametric Chirped-Pulse Amplification."

J. D. Zuegel, J. R. Marciante, A. Galvanauskas, and C.-H. Liu, "High-Energy Fiber Power Amplifier for Broadband Beam Smoothing with FM-Modulated Laser Pulses on OMEGA."
The following presentations were made at the 5th International Conference on High Energy Density Laboratory Astrophysics, Tucson, AZ, 10-13 March 2004:

T. R. Boehly, E. Vianello, J. E. Miller, R. S. Craxton, V. N. Goncharov, D. D. Meyerhofer, T. C. Sangster, D. G. Hicks, and P. M. Celliers, "Laser-Driven, Multishock Experiments in Planar Targets."

S. P. Regan, T. C. Sangster, D. D. Meyerhofer, K. Anderson, R. Betti, T. R. Boehly, T. J. B. Collins, R. S. Craxton, J. A. Delettrez, R. Epstein, O. V. Gotchev, V. Yu. Glebov, V. N. Goncharov, P. A. Jaanimagi, J. P. Knauer, J. A. Marozas, F. J. Marshall, P. W. McKenty, P. B. Radha, W. Seka, S. Skupsky, H. Sawada, V. A. Smalyuk, J. M. Soures, C. Stoeckl, B. Yaakobi, J. A. Frenje, C. K. Li, R. D. Petrasso, and F. H. Séguin, "Direct-Drive Inertial Confinement Fusion Implosions on OMEGA." 
\begin{tabular}{l} 
ROCHESTER \\
\hline
\end{tabular} 